\title{
Ecology of Trifolium stoloniferum (Muhl. ex A. Eaton), a federally endangered vascular plant, at the Fernow Experimental Forest in West Virginia
}

John Q. Burkhart

West Virginia University

Follow this and additional works at: https://researchrepository.wvu.edu/etd

\section{Recommended Citation}

Burkhart, John Q., "Ecology of Trifolium stoloniferum (Muhl. ex A. Eaton), a federally endangered vascular plant, at the Fernow Experimental Forest in West Virginia" (2010). Graduate Theses, Dissertations, and Problem Reports. 2169.

https://researchrepository.wvu.edu/etd/2169

This Thesis is protected by copyright and/or related rights. It has been brought to you by the The Research Repository @ WVU with permission from the rights-holder(s). You are free to use this Thesis in any way that is permitted by the copyright and related rights legislation that applies to your use. For other uses you must obtain permission from the rights-holder(s) directly, unless additional rights are indicated by a Creative Commons license in the record and/ or on the work itself. This Thesis has been accepted for inclusion in WVU Graduate Theses, Dissertations, and Problem Reports collection by an authorized administrator of The Research Repository @ WVU. For more information, please contact researchrepository@mail.wvu.edu. 
Ecology of Trifolium stoloniferum (Muhl. ex A. Eaton), a federally endangered vascular plant, at the Fernow Experimental Forest in West Virginia

John Q. Burkhart

\author{
Thesis submitted to the \\ Davis College of Agriculture, Natural Resources and Design \\ at West Virginia University \\ in partial fulfillment of the requirements \\ for the degree of
}

Master of Science

In

Forest Resources Management

James S. Rentch, Ph.D., chair

Kathryn B. Piatek, Ph.D.

Thomas M. Schuler, Ph.D.

David W. McGill, Ph.D.

Donna I. Ford-Werntz, Ph.D

Divison of Forestry and Natural Resources

Morgantown, West Virginia

2010

Keywords: Trifolium stoloniferum, running buffalo clover, Fernow Experimental Forest, disturbance, endangered species, skid roads

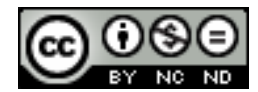

2010 John Q. Burkhart

Creative Commons Attribution Non-Commercial No-Derivatives 3.0 Unported License 


\section{Abstract \\ Ecology of Trifolium stoloniferum (Muhl. ex A. Eaton), a federally endangered vascular plant, at the Fernow Experimental Forest in West Virginia}

\section{John Q. Burkhart}

This thesis presents work addressing the ecology of Trifolium stoloniferum, a federally endangered vascular plant species, in the Fernow Experimental Forest, West Virginia. In this thesis, I describe the historical ecology of this species and make a case that at one time it occurred in great abundance in association with trails created by large mammals and humans that intersected rich, open forests. Similar conditions exist at the Fernow Experimental Forest in West Virginia, but instead of large mammals, the requisite soil disturbance, control of competing vegetation, and canopy perforation are created by timber-harvesting related disturbances, particularly gap creation by tree felling and skidding of trees from the forest.

I conducted two distinct studies to quantitatively and qualitatively describe the habitat conditions that promote $T$. stoloniferum success. The first study occurred at the scale of a forested stand. At the level of the stand, total number of logging-related disturbances since 1945 was the most important characteristic in determining the presence or absence of the species, with greater number of disturbances strongly related to the presence of the species. Time since last disturbance and aspect interacted to affect $T$. stoloniferum density within a stand, with westfacing stands that had been disturbed more recently than 14.5 years supporting the greatest densities of $T$. stoloniferum. This study revealed that stands managed in uneven-aged silvicultural systems with frequent management entrances that also received high levels of light were most capable of supporting vigorous occurrences of $T$. stoloniferum.

The second study consisted of a detailed habitat assessment of $T$. stoloniferum patches. I stratified patches at the Fernow Experimental Forest based upon patch abundance and inflorescence production and conducted detailed habitat assessment of a representative sample of patch sizes and relative inflorescence production. I assessed the vegetation, substrate, physiography, and localized disturbance history, and also took canopy photographs using a hemispherical lens. Patch abundance was the result of a suite of interactions between canopy structure, tree basal area, and disturbance history. Abundant sites also had high diversity of associated herbaceous species, suggesting that good sites for T. stoloniferum are also good for a suite of early and mid-successional forest herbs. Inflorescence production was the consequence of light levels, with high light levels associated with increased inflorescence production.

The management and conservation of Trifolium stoloniferum should focus on maintaining and encouraging those processes and activities that periodically disturb soil and create light gaps in mixed, mesophytic forests. In addition to the deliberate efforts of managers, incidental management opportunities, or situations in which the promotion of T. stoloniferum is an unintended outcome, should be identified and fostered. 


\section{Acknowledgements}

Many people have offered their time, encouragement, knowledge, and financial assistance in making this project successful. I thank the kind men and women who work at the Fernow Experimental Forest for helping me feel at home as I conducted my field work and for providing lodging. I would like to thank Frederica Wood for GIS assistance and Darlene Madarish for assembling a useful guide to the locations of Trifolium. stoloniferum at the Fernow. I thank Dr. Cindy Huebner of the United States Forest Service for her generous assistance in the design of vegetation sampling. I thank my wife, Aira Loren Burkhart, for her drawings of $T$. stoloniferum and the field day she spent with me to acquaint herself with the species. I sincerely thank Dr. Thomas Schuler for providing wise counsel, stimulating discussion, and the opportunity and resources to conduct this study. I would like to offer my sincerest gratitude to Dr. Kathryn Piatek for getting me to WVU and being a gracious and understanding advisor. Dr. James Rentch deserves special recognition for becoming my advisor during the middle of the process and providing assistance during the statistical analysis process. I thank my committee members Dr. Dave McGill and Dr. Donna Ford-Werntz for their useful comments on my manuscript. Funding for this study was provided by United States Forest Service Cooperative Agreement with West Virginia University 09-JV-11242301-132: "Promoting expansion and flourishing of the endangered Running Buffalo Clover- determination of optimal light levels and agents of disturbance." 


\section{Table of Contents}

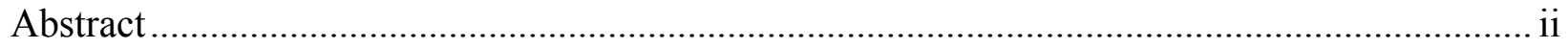

Acknowledgements .......................................................................................................ii

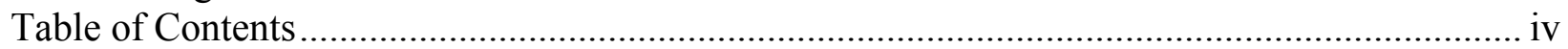

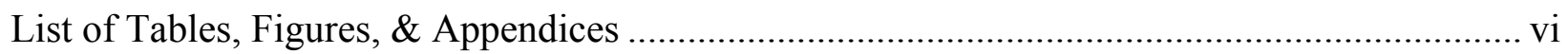

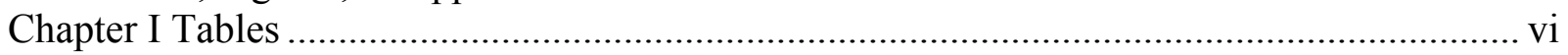





Chapter II Tables .......................................................................................................... vi

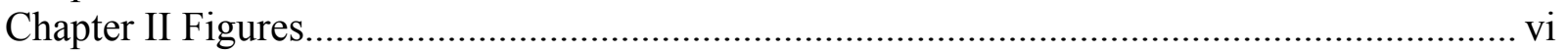

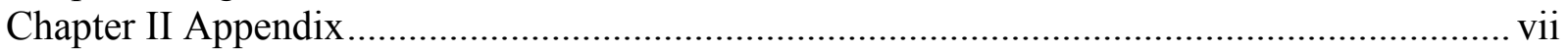

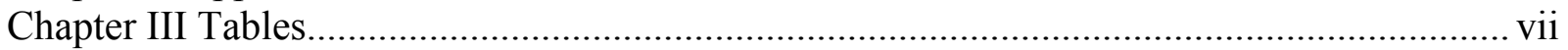

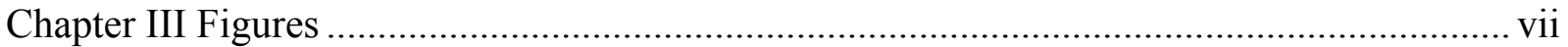

Chapter III Appendix ……........................................................................................... viii

Chapter I: Trifolium stoloniferum: An ecological history, review of contemporary research, and introduction of original research questions and goals.............................................................. 1

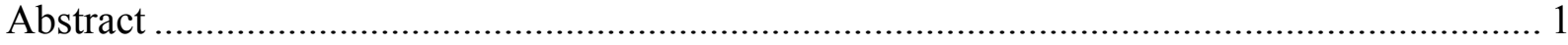



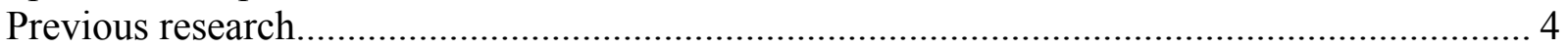

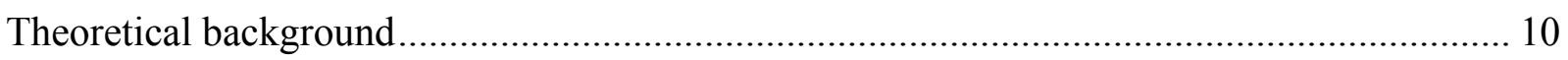

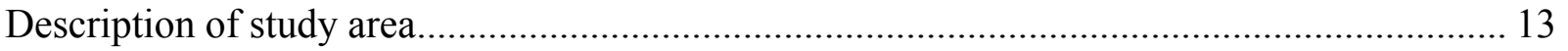

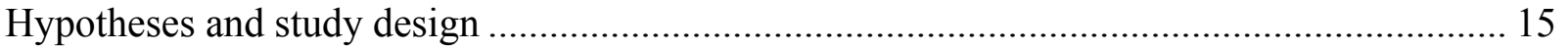

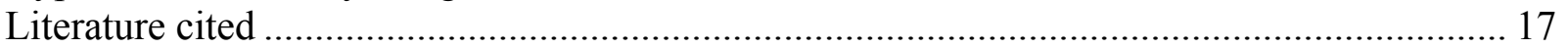



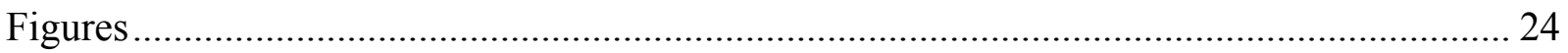

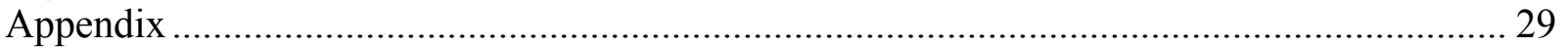

Chapter II: Effects of forest management on running buffalo clover (Trifolium stoloniferum) distribution and abundance in the Fernow Experimental Forest ................................................. 32

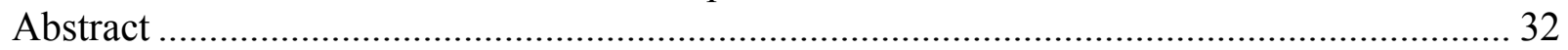

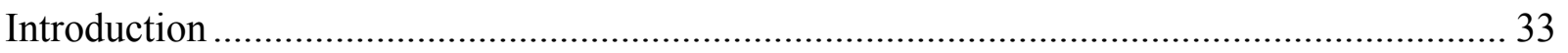

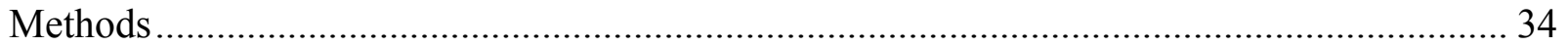

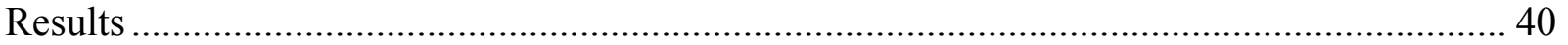

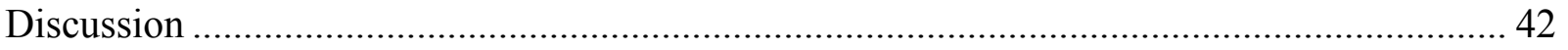

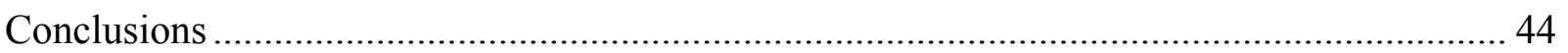

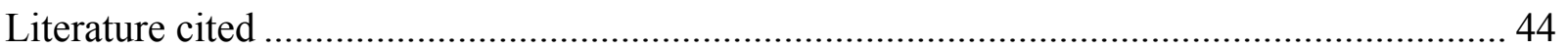

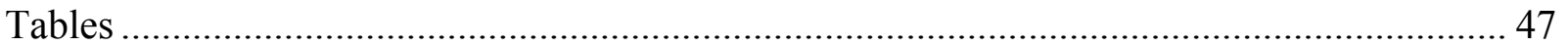

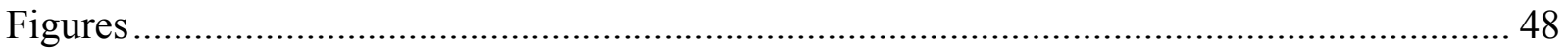

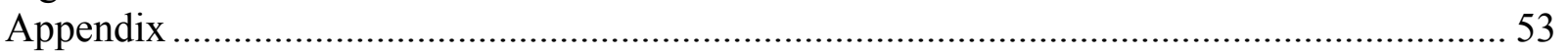

Chapter III: Habitat assessment of Trifolium stoloniferum: Relationship of patch abundance and

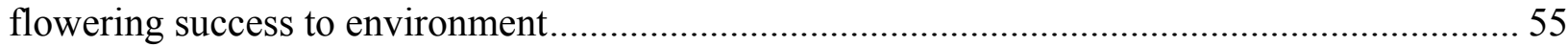



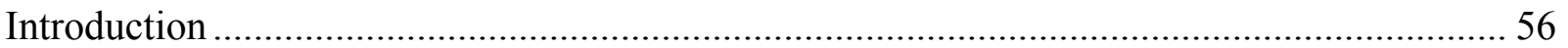

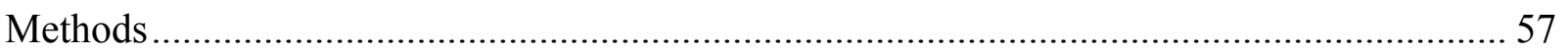

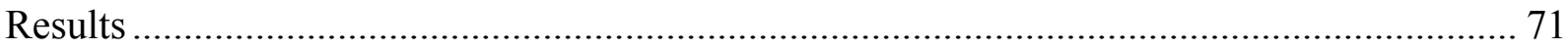




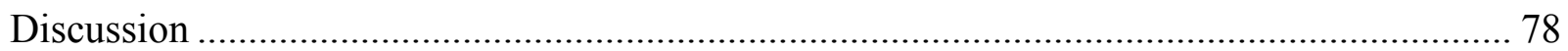

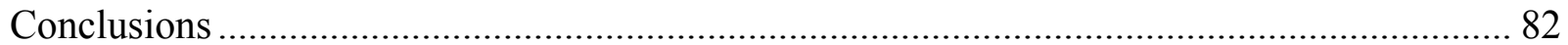

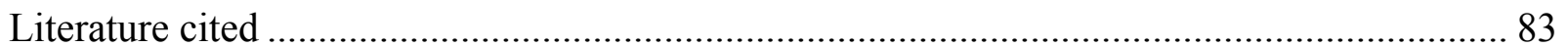



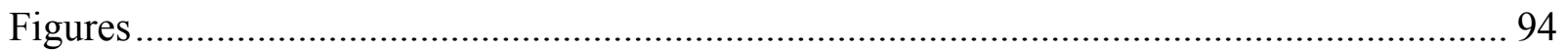

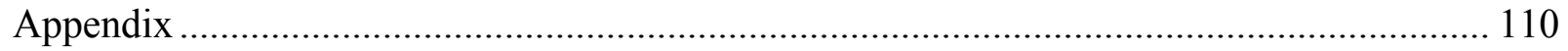

Chapter IV: Summary of findings and implications for management of Trifolium stoloniferum

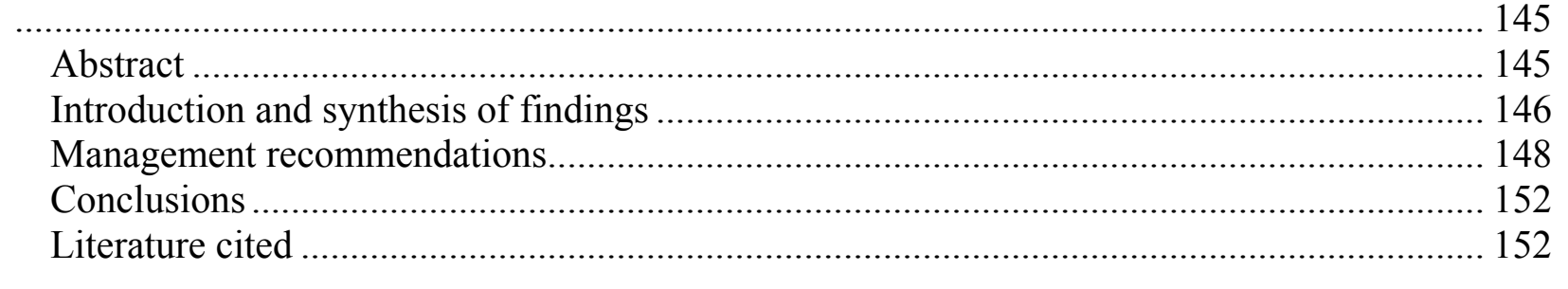




\section{List of Tables, Figures, \& Appendices}

Table, figure, and appendix captions are abbreviated in this list for the sake of clarity and conciseness.

\section{Chapter I Tables}

Table 1. Herbs found in association with Trifolium stoloniferum and the mean $\%$ cover of that species measured in habitat assessment plots................................................................... 20 Table 2. Shrub cover by species and the mean distance that each shrub species intersect with the transect in habitat assessment plots. 22

Table 3. Mean basal area of trees in $\mathrm{ft}^{2} / \mathrm{ac}$ as measured during the habitat assessment process for T. stoloniferum. 23 Table 4. Mean number of saplings (trees $<10 \mathrm{~cm}$ in diameter at breast height) found inside the $100 \mathrm{~m}^{2}$ habitat assessment plots.

\section{Chapter I Figures}

Figure 1. Photograph of Trifolium stoloniferum .................................................................... 24

Figure 2a. Illustration of Trifolium stoloniferum ............................................................... 25

Figure 2b. Close-up of T. stoloniferum inflorescence............................................................ 26

Figure 3. Extant distribution of Trifolium stoloniferum.................................................... 27

Figure 4. Phylogenetic tree of the genus Trifolium.............................................................. 28

\section{Chapter I Appendix}

Appendix 1. Geographic names using "Buffalo" in West Virginia and parts of surrounding states

\section{Chapter II Tables}

Table 1. Mean of predictor variables when RBC is present or absent.

\section{Chapter II Figures}

Figure 1. Classification tree analysis of $T$. stoloniferum presence or absence ........................ 48 Figure 2. Classification tree analysis of $T$. stoloniferum density category as the response

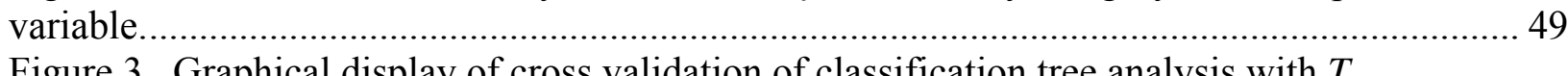
Figure 3. Graphical display of cross validation of classification tree analysis with $T$. stoloniferum density category as the response variable ................................................ 50 Figure 4. Regression tree analysis with median T. stoloniferum as response variable............... 51 Figure 5. Graphic display of discriminant function analysis with T. stoloniferum presence or absence as the categorical predictor variable and the influence of the predictor variables upon the response. 


\section{Chapter II Appendix}



\section{Chapter III Tables}

Table 1. Means and standard deviations of environmental variables by Crowngroup ............... 86

Table 2. Means and standard deviations of environmental variables by Flowergroup. ..............8 87

Table 3. Comparison between control sites that used to contain T. stoloniferum and sites that



Table $4 \mathrm{a}$ and $\mathrm{b}$. Results of MANOVA analyses performed with Crowngroup and Flowergroup as predictive factor and collective environmental variables as the multivariate response. .......... 88 Table 5. Results of environmental fitting function performed on nonmetric multidimensional scaling ordination of herbaceous communities measured in T. stoloniferum and control sites.... 89 Table 6. Summary statistics of multi-response permutation procedure (MRPP) with Crowngroup as the a priori grouping.

Table 7. Pairwise comparisons of Crowngroups based upon findings of MRPP. P-values have been adjusted for multiple comparisons. .......................................................................... 90 Table 8. Summary statistics of multi-response permutation procedure (MRPP) with Flowergroup as the a priori grouping. 91

Table 9. Pairwise comparisons of Flowergroups based upon findings of MRPP. The p-values have not been corrected for multiple comparisons. ....................................................... 91

Table 10. Results of indicator species analysis for Crowngroup ............................................. 92

Table 11. Results of indicator species analysis for Flowergroup. ....................................... 93

\section{Chapter III Figures}

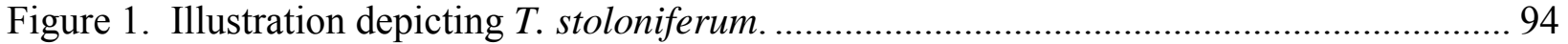

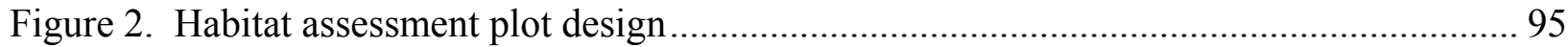

Figure 3. Crowngroups as labels: Environmetal variables as vectors. .................................... 96

Figure 4. Flowergroups as labels: Environmental variables as vectors ................................... 97

Figure 5. Crowngroups as labels: Time since last disturbance (TSLD) as surface. ................. 98

Figure 6. Crowngroups as labels: Shannon's diversity index as surface ................................ 99

Figure 7. Crowngroups as labels: Canopy openness (OPEN) as surface.. ............................ 100

Figure 8. Crowngroups as labels: Basal area (BA) as surface .............................................. 101

Figure 9. Flowergroups as labels: Shannon's diversity index (SHANNON) as surface.......... 102

Figure 10. Flowergroups as labels: Time since last disturbance (TSLD) as surface............... 103

Figure 11. Flowergroups as labels: Basal area (BA) as surface ............................................ 104

Figure 12. Flowergroups as labels: Shrub cover (SHRUBCOV) as surface. ......................... 105

Figure 13. Flowergroups as labels: Photosynthetic photon flux density (PPFD) as surface.... 106

Figure 14. Classification tree analysis with Crowngroup as the response and Shannon's diversity

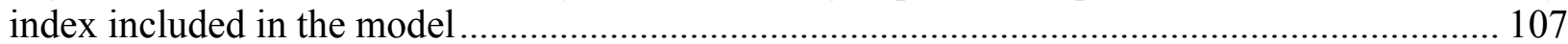

Figure 15. Classification tree analysis with Crowngroup as the response and Shannon's diversity index excluded from the model. 
Figure 16. Classification tree analysis with Flowergroup as response variable .....

\section{Chapter III Appendix}



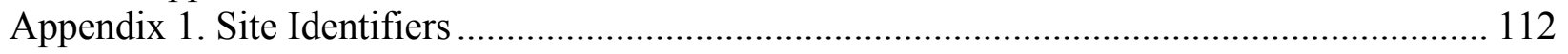

Appendix 2. Photographs and descriptions of study sites............................................... 114 


\title{
Chapter I
}

\section{Trifolium stoloniferum: An ecological history, review of contemporary research, and introduction of original research questions and goals}

\begin{abstract}
The conservation of Trifolium stoloniferum, or running buffalo clover, presents management challenges novel to many managers and conservation scientists. This species requires disturbance to both soil and canopy in order to flourish, and this disturbance may be difficult for many managers to replicate or potentially contrary to other conservation goals, such as invasive species control and wilderness preservation. Nonetheless, disturbance is necessary and compromise solutions will be needed in order to conserve this species. In this chapter, I survey previous studies of this species and demonstrate the need for research into its basic environmental requirements. Previous work has focused upon the population genetics, soil chemistry, and dispersal biology of this species, but relatively few research efforts have focused upon clearly delineating the environmental conditions requisite for population and patch success. I put forth the hypotheses that guide my research into the ecology of this species and explain the reasoning which contributed to the formation of these hypotheses using ecological theory, particularly the intermediate disturbance hypothesis and supporting species-specific information. Keywords: Trifolium stoloniferum, intermediate disturbance hypothesis, clover, forest disturbance.
\end{abstract}

\section{Species description}

Trifolium stoloniferum Muhl. ex Eaton, commonly known as running buffalo clover (or $\mathrm{RBC}$ ), is a species native to eastern North America (Figures 1 and 2). Its extant distribution 
ranges east to the Allegheny Front of West Virginia and west to the Missouri Ozarks (Figure 3), and is found as well in Indiana, Ohio, and Kentucky (USFWS 2007). Historical, specimenvalidated records are present from Arkansas, Illinois, and Kansas. Trifolium stoloniferum is usually, but not always, found on soils at least partially derived from limestone and other calcareous bedrock in habitats that receive filtered sunlight and experience a moderate, periodic disturbance regime. The exceptions to this habitat description often appear to be strays or highly ephemeral populations (USFWS 2007). Higher-quality habitats include but are not limited to rich, mesic forests that are periodically disturbed by logging, foot travel, or animal use, trails and other disturbed areas in river floodplains, cemeteries, lawns, forested savannahs, sandbars, and wildlife openings within rich, mature forests that are periodically mowed (Campbell et al. 1988, USFWS 2007).

Based upon accounts by early explorers of the region in which T. stoloniferum is found, as reported in Campbell et al. (1988), the ancestral habitat of T. stoloniferum is reasoned to be paths and small clearings created and maintained by woodland bison (Bison bison athabascae Rhoads) and other large game, such as Eastern elk (Cervus canadensis Erxleben canadiensis ) and white-tailed deer (Odocoileus virginianus Zimmerman) in forested settings. Additionally, the management activities of Native Americans, especially intentional fires (Jakle 1968, Campbell et al. 1988, Nowacki \& Abrams 2008), were likely associated in maintaining habitats favorable for this species. This description of the ancestral habitat is widely accepted, and is supported by historical accounts, observations of the extant habitats of T. stoloniferum, and by the common name, which reflects early European explorers of this region association of the species with trails created and maintained by the activity of bison (Campbell et al. 1988, USFWS 2007). 
That $T$. stoloniferum was once widespread and at least locally abundant seems possible and likely based upon historical accounts (Campbell et al. 1988) and ecological reconstructions (Jakle 1968) which depict extensive swaths of land suitable for the growth of the species, particularly throughout the limestone underlain Bluegrass region of Kentucky. The local or functional extinctions of those human and animal populations that made trails, maintained openings, and dispersed seeds have likely contributed to the decline of $T$. stoloniferum.

Based upon archaeological evidence, it is likely that woodland bison experienced dramatic increases in abundance and eastward range expansion around A.D.1500 throughout the Ohio Valley and into the mountainous Appalachian region (Jakle 1968, Smith 1989). Bison abundance had grown during the Hopewellian moundbuilder period (A.D. 500-1400) during which agriculture was emphasized more than migratory hunting. But woodland bison abundance and range expansion reached its zenith after the arrival, around A.D. 1500, of the Algonquin native peoples who brought with them their extensive use of fire to create and maintain grasslands (it is highly likely that other native people of the region used fire for management reasons, but the Algonquin people expanded the role of fire in the region). As bison expanded eastward, they created extensive systems of "buffalo traces" which often connected salt licks and other important resources, such as cane breaks (Arundinaria gigantea (Walt.) Muhl.). These buffalo traces were also important migration corridors for early European settlers, and many important early settlements were located at the junction of buffalo traces (Jakle 1968). The importance of woodland bison throughout the range of Trifolium stoloniferum is reflected in the names of geographical features and places (for examples, see Appendix 1). Early settlers who took notice of the vegetation often remarked at the great abundance of "flowering white clovers" along the buffalo traces and in the wooded glades that were frequented by the bison (Campbell 
1988). The woodland bison was rapidly hunted to extinction whenever it came into contact with the arriving European settlers; the migratory herds were often ambushed at salt licks (Jakle 1968). The loss of the woodland bison and the Native Americans who maintained habitats for the bison is likely to have set the stage for a decline in the plant and animal species adapted to the particular habitat conditions created by their activities. Among these species was $T$. stoloniferum.

Additionally, destruction and modification of habitat, land-use changes such as conversion of forests and savannahs to intensive agricultural production, changes in forest structure after the arrival of European settlers, and competition with non-native and native plants have contributed to its decline (USFWS 2007). In effect, a whole suite of environmental and ecological changes initiated and maintained by the expansion of European peoples in North America have synergistically interacted to contribute to the decline of T. stoloniferum. Its decline was so dramatic that it was presumed extinct or extremely rare by Brooks (1983), who found no records or accounts of this species after 1940. But in 1985 Bartgis reported the rediscovery of this species after finding two small populations in West Virginia in Webster and Fayette counties (Bartgis 1985). Subsequent search efforts revealed additional extant populations in West Virginia and other states, including Indiana, Ohio, and Kentucky (Cusick 1989, Homoya et al. 1989, USFWS 2007). The species was listed as endangered in 1987 (USFWS 1987) and initial (USFWS 1989) and revised (USFWS 2007) recovery plans were drafted and approved.

\section{Previous research}

There have been several observational and experimental research studies of $T$. stoloniferum to date. The outcomes of these investigations have revealed unique or interesting 
biological properties of $T$. stoloniferum and have contributed significantly to understanding of the ecology and management of this species. The scholarly research published to date relevant to my current research efforts can be grouped loosely into the following categories: (1) genetics (Hickey et al. 1991, Crawford et al. 1998), (2) reproductive biology and dispersal (Franklin 1998, Ford et al. 2003), (3) characteristics of habitat, soil, and nitrogen-fixing ability (Hattenbach 1996, Morris et al. 2002, Madarish \& Schuler 2002) (4) history, distributional notes, and rediscovery (Brooks 1983, Bartgis 1985, Davis 1987, Campbell et al. 1988, Cusick 1989, Homoya et al. 1989), and (5) potential pathogens (Sehgal \& Payne 1995, Quesenberry et al. 1997).

The first study to address genetic variation within and among wild populations of running buffalo clover was Hickey et al. (1991), which investigated allozyme diversity in two native and two exotic Trifolium species. Allozymes are variant forms of an enzyme that are coded for by different alleles that occur at the same loci. They reported that overall, $T$. stoloniferum did not possess substantial diversity as measured by allozyme diversity, as only $15 \%$ of loci were polymorphic, and the average number of alleles was 1.10 per loci, indicating a great deal of homozygosity. Hickey et al. also suggested that a high proportion, approximately $34 \%$, of genetic diversity was found between populations rather than within. In this study they also reported that gene flow was relatively low even among populations that were geographically very close and that small populations were particularly lacking in allozyme diversity. They interpreted their findings of relatively low levels of genetic diversity within and among populations with guarded optimism, given that at their time of publication, T. stoloniferum had been but recently rediscovered and even low measures of genetic diversity were encouraging. 
Crawford et al. (1998) used a more advanced technique to measure genetic diversityRandom Amplified Polymorphic DNA's (RAPDs). They pointed out that allozyme diversity might be inherently low or nonexistent in rare plants; thus, even if genetic diversity exists within and among populations, it might not be detected in the form of enzyme diversity. RAPDs measure genetic diversity in a form that might be more variable and less conserved than allozymes- i.e., RAPDs are more sensitive. Crawford et al. found that within a population it is likely that two rooted crowns are of a different genotype, but that there is high substructuring of subpopulations, which is expected since the growth habit of $T$. stoloniferum is true to its specific epithet. They further found that even small populations are likely to be relatively diverse, that greater diversity existed between populations rather than within, and that populations from within each geographic area sampled were more similar to each other than populations from different geographic areas. Crawford et al. concluded that both small and large populations contained ecologically and evolutionarily significant genetic diversity, and that conservation efforts should focus on retaining and expanding representative small populations in addition to large populations.

The reproductive biology of $T$. stoloniferum was the focus of the Master's thesis of Franklin (1998), who found that T. stoloniferum was capable of producing viable seeds that grew into healthy plants by means of self-pollination. She also found that seeds, produced by both selfing and outcrossing, from a smaller population were higher quality, as measured by seed size and viability, than those from a larger population; however, she concluded that this was most likely the result of differences in habitat characteristics, particularly light levels reaching the plants. Ford et al. (2003) investigated the potential of white-tailed deer to function as dispersers of running buffalo clover. After feeding seeds to a deer, gathering those that passed through the 
gut, subjecting $1 / 2$ of the remaining seeds to cold-scarification and not further manipulating the remaining $1 / 2$, they planted the seeds and measured germination. They also planted seeds that had never passed through a deer's gut and scarified $1 / 2$ of those seeds. They found that a relatively small proportion of the seeds survived the deer's gut, and surviving seeds did not germinate better than other treatments. Thus, deer, which today are the most abundant ungulate throughout the range of T. stoloniferum, do not appear to be a significant dispersal vector for this species. At the Fernow Experimental Forest in West Virginia, timber-harvesting equipment moving over skid roads appears to be the most important source of seed dispersal (Madarish \& Schuler 2002). Hattenbach (1996) also observed that seeds with the highest degree of scarification in an acidbath had the highest rates of germination. A thorough understanding of the mechanisms of dispersal and germination for $T$. stoloniferum has not yet been achieved, particularly means of dispersal and germination that are not implemented by humans.

The characteristic habitat of $T$. stoloniferum is a periodically and moderately disturbed site in a mesic habitat that receives sufficient filtered or dappled sunlight. This can include a wide variety of specific community types- upland and floodplain deciduous forests, savannahs, mowed cemeteries and lawns, and wildlife openings, among others (USFWS 2007). Trifolium stoloniferum has been characterized as preferring limestone-derived soils, and although there are many exceptions this generalization is still useful in guiding searches for new populations. It is rarely found in association with acidophilic species such as members of the family Ericaceae (USFWS 2007). Hattenbach (1996) characterized the soil chemistry and particle size around $T$. stoloniferum sites throughout its range and found substantial variation in the soil chemistry around the plants, but the species was clearly not associated with acidic soils and found usually in moderately basic to circumneutral soils. A study of the effects of logging disturbace on $T$. 
stoloniferum by Madarish \& Schuler (2002) at the Fernow Experimental Forest found populations recovered after being disturbed by log skidding operations, providing evidence that this species is adapted to trampling, admixing, and other soil disturbances. They also reported that the canopy above T. stoloniferum patches had a greater gap fraction and a lower leaf area index than areas in the same forest management unit that did not have T. stoloniferum.

The rediscovery of $T$. stoloniferum by Bartgis (1985) came after a summary publication by Brooks (1983) which fully and technically described the species, listed the locations from which it had been collected or validly reported, and offered informed speculation as to the demise and current distributional status of the species. In this publication, Brooks (1983) cogently observes “...that the habitat destruction resulting from the industrial revolution and the inability of $T$. stoloniferum to adapt to changing environmental stresses led to the demise of this species." However, Brooks suggests that this species was perhaps inherently rare. Campbell et al. (1988), studying accounts of early explorers, dismisses this hypothesis and contends that in recent (i.e., post-Columbian) ecological history, $T$. stoloniferum had most likely been the most abundant and widespread member of the genus Trifolium native to eastern North America. Cusick (1989) elaborated further on the distribution and habitat description of T. stoloniferum in Ohio. Cusick confirms a general and abundant occurrence of $T$. stoloniferum in historical accounts in pre-1800 and early 1800's accounts. The habitats of $T$. stoloniferum are described as usually found associated with fluvial terraces in mesic sites receiving filtered sunlight that had been repeatedly and historically disturbed by mowing, trampling, grazing, etc.

Research into potential pathogens of $T$. stoloniferum has suggested that neither disease nor predation represent a substantial, range-wide threat to wild populations of running buffalo clover (USFWS 2007). Quesenberry et al. (1997) inoculated T. stoloniferum grown in a 
greenhouse setting with root-knot nematodes and found them to have high resistance to infection. Isolated populations of $T$. stoloniferum have been reported to be eaten by a wide variety of herbivores, but no workers have observed consistent, destructive predation of this species (USFWS 2007).

Related research which illuminates some aspects of the biology of running buffalo clover includes the molecular phylogenetic history and tree of the genus Trifolium assembled by Ellison et al. (2006). Their work recognizes T. stoloniferum as the basal-most, or the least derived evolutionarily, species of a small clade of clovers, all of which are native to the eastern or southern United States (Figure 4). Along with the other members of this clade it lacks functional rhizobial associates, and thus does not fix nitrogen in any significant way (see Morris et al. 2002). Ellison et al. (2006) also emphasized that all species of Trifolium are not especially shade-tolerant and need moderate levels of direct sunlight in order to flower.

Research on running buffalo clover has focused on describing past abundance, distribution, and ancestral habitats (Campbell et al. 1988), genetic diversity within and among populations (Crawford et al. 1998), its breeding system (Franklin 1998), characteristics of its soil environment (Hattenbach 1996), effects of herbivory upon germination success (Ford et al. 2003), and the effects of disturbance and forest management upon running buffalo clover (Madarish and Schuler 2002). The USFWS Recovery Plan (2007) for T. stoloniferum emphasizes the necessity of research into fundamental aspects of the ecology of the species. More specifically, the authors of the recovery plan highlight the importance of understanding the factors that control population size and reproductive success. Sexual reproduction, which promotes adaptive response to changing environments, is critical to the long-term viability of 
T. stoloniferum, and thus to manage it effectively it is essential to identify the environmental conditions which foster sexual reproduction (USFWS 2007).

\section{Theoretical background}

The theoretical concepts which have most influenced the design and interpretation of my experiment are the intermediate disturbance hypothesis, or IDH (Hutchinson 1953, Grime 1973, Connell 1978), and various concepts put forth in plant ecology literature relating plant species richness to successional and disturbance patterns (e.g., Denslow 1980, McIntyre et al. 1999). The applicability of the IDH to the conservation of a single species could be questioned, as this hypothesis was conceptualized as a mechanism to permit the coexistence of species within a given space by means of disturbance creating either temporal or spatial environmental heterogeneity or both. However, if we consider T. stoloniferum to be a member of a successional plant community that benefits from disturbances that are intermediate in both intensity and frequency, then the application of the IDH to guide hypothesis formation and experimental design is both useful and appropriate.

The IDH is often characterized as a trade-off between dispersal/reproductive capacity and competitiveness: ruderal species can reproduce successfully in environments modified by disturbance but are eventually suppressed by the more competitive members of the community as succession progresses. At an intermediate level of disturbance, the coexistence of species with varied life histories is maximized (Roxburgh et al. 2004). Roxburgh et al. and others (e.g., Chesson 2000) have expanded the initial conceptions of the IDH, which generally referred to mechanisms that permitted the spatial coexistence of species, to also include mechanisms that permit temporal coexistence of species. Roxburgh et al. suggest that the IDH is deceptively simple, but the notion of "intermediate disturbance" has been used to represent a broad range of 
phenomena in varied ecosystems. The nature of what constitutes an "intermediate" disturbance eludes universal generalization, and the definition of intermediate is usually environmentspecific and must be referenced to the prevailing disturbance regime.

Life history strategies, such as increased dispersal ability, seed banking, and rapid germination allow for a species to take advantage of ephemeral successional habitats. Denslow (1980) suggested that the plant species inhabiting a forest have evolved and express life-history traits that afford them greatest success, in terms of growth and reproduction, in different successional states. In other words, forest environments are "patchy," or environmentally heterogenous in both space and time, and species can successfully establish, grow, and reproduce best in certain forest conditions that are the outcome of the interactions between disturbance, succession, and potential vegetation. In the case of $T$. stoloniferum, both temporal and spatial coexistence appear to be relevant in maintaining this species within a patch and at larger spatial scales. Patches of suitable habitat are created and maintained by the same processes that may disperse seeds- forestry operations such as skidding and road creation, mowing, and dispersal in the guts, coats, and hooves of large animals. In addition to habitat creation, these same activities may maintain habitat conditions to be suitable for Trifolium stoloniferum.

McIntyre et al. (1999) set forth a general pattern of the response of plants to disturbance based upon functional traits and morphology and reviewed general trends. Following their descriptions of plant life history strategies $T$. stoloniferum appears to be adapted to moderate disturbances to its potential and actual habitat. While heavy disturbances favor annuals that produce large amounts of seed that are often stored in the seed bank, limited disturbances tend to favor more competitive perennials, who are less likely to be successful at storing seed in the seed bank. Trifolium stoloniferum falls in the middle of this spectrum, as it is a short-lived perennial 
and capable of contributing seeds to a seed bank and regenerating from underground root systems (USFWS 2007). It is capable of germinating in areas characterized by light to moderate disturbances, but it is not able to effectively compete with long-lived forest perennials in closedcanopy forests, nor does it appear to thrive in areas of extreme disturbance, such as clearcuttings. Unfortunately, little is known about the viability and longetivy of $T$. stoloniferum seeds in the seed bank.

The disturbances to which T. stoloniferum has adapted can be described as intermediate in frequency and intensity. Van der Maarel (1993) suggests that the destruction of biomass is an integral component of disturbance. Disturbances which maintain T. stoloniferum across its geographic range vary widely in causal mechanism, but they all share the commonality of partial removal or destruction of the biomass of that system. In the case of T. stoloniferum, it appears that disturbance to the soil is requisite for establishment, and that disturbance to canopy or maintenance of open canopy conditions is requisite for growth and flowering.

Huennekke \& Hobbs (1992) highlight the potential downsides of disturbance, including increased invasion by non-native species, the difficulties of deciding upon and implementing appropriate disturbance regimes, and potential loss of habitat for some species. In most realworld management situations, these concerns will have to be balanced with goal-based conservation objectives, such as the promotion of rare or endangered species and the need to maintain ecosystem services. The disturbances that have maintained $T$. stoloniferum populations to this date have largely been unrelated to the conservation of this species. Periodic grazing, logging, mowing, and other forms of disturbance intermediate in frequency and intensity were largely used to achieve management objectives that were incidental to or oblivious of this species. In all reality, disturbances around established populations may have lessened due to the 
actions that managers took to protect the few known populations (PJ Harmon, personal communication). The unique position of my study site- the Fernow Experimental Forest- as an active research forest with management actions prescribed for forest stands into the indefinite future does not allow for complete protection of $T$. stoloniferum, but it did allow for populations to become established, be mapped and inventoried, and for precise records of management activities to be recorded. As will be revealed in this thesis, certain intensities and frequencies of management activities at the Fernow have promoted the establishment and growth of $T$. stoloniferum occurrences. Now, as of 2010, we realize that there are sufficient abundances of $T$. stoloniferum to permit limited experimental manipulation of habitats in an attempt to promote the species and restore it to its full potential as a member of our native flora. It is the main goal of this thesis to accurately and thoroughly portray the environmental "scene" which offers $T$. stoloniferum its best opportunity to flourish and thus to inform its management and conservation.

\section{Description of study area}

The Fernow Experimental Forest in West Virginia (latitude $39^{\circ} 3^{\prime} 15^{\prime \prime} \mathrm{N}$, longitude $79^{\circ} 41^{\prime} 15^{\prime \prime} \mathrm{W}$ ) is a research forest established in 1934 approximately $2.5 \mathrm{~km}$ south of the town of Parsons, WV (Madarish et al. 2002). The Fernow contains 1902 ha of largely forested habitat ranging from 533 to $1,112 \mathrm{~m}$ above sea level. It is a part of the Monongahela National Forest, an approximately $3,719 \mathrm{~km}^{2}$ forest stretching southwest to northeast across the mountainous regions of West Virginia.

Silvicultural, ecological, and watershed research has been conducted on the Fernow Experimental Forest since 1934 (Madarish et al. 2002). A large component of the Forest's mission has been to implement and maintain silvicultural practices at the level of the management unit within the forest. The fundamental unit of management at the Fernow is either 
the watershed or compartment- with compartments having been delineated based on site characteristics. Compartments are in some cases further divided into subcompartments, and silvicultural treatments are applied at either this level or at the compartment level.

Trifolium stoloniferum is found in approximately 67 extant "patches" in 15 subcompartments or watersheds. All but one of the patches occurs in a subcompartment or watershed underlain by calcareous soils. The disturbance that appears to have been most influential in maintaining T. stoloniferum is periodic logging and associated skidding. Trifolium stoloniferum is most likely dispersed by the logging equipment as well, as $T$. stoloniferum is found largely along skid roads that are used episodically (at intervals from less than 10 to 50 years) to remove timber from the forest.

The species of herbs, shrubs, and trees found in association with T. stoloniferum at the Fernow Experimental Forest are presented in Tables 1, 2, and 3, respectively (the process of sampling and measuring associated vegetation is described thoroughly in Chapter III of this thesis). I have limited the herbaceous species presented to those that had greater than approximately $0.05 \%$ mean cover across all habitat assessment plots. Associated herbaceous and shrub species are affected by the same ecological processes that affect T. stoloniferum: disturbance to the soil and canopy, light, and competition from their neighbors, and site characteristics such as soil chemistry and composition, topographic position, and microenvironmental variation. The potential species pool is affected by a myriad of biogeographic and ecological processes, including human activities. Tree species composition and structure is a function of the potential species pool of the region and its interaction with historical land-use, topographic and edaphic factors, and the research-driven forestry activities that are carried on at the Fernow Experimental Forest (Schuler 2004). 


\section{Hypotheses and study design}

Thomas M. Schuler, Ph.D., Research Forester at the Fernow Experimental Forest, presented me with two questions at the inception of this project. First, "why does running buffalo clover thrive in some patches and not in others?" Second, "Why does running buffalo clover flower abundantly in some patches and not in others?" Pilot field work and exploratory data analysis, literature review, and conversations with Dr. Schuler suggested that light penetration to the herb level, disturbance frequency, composition and structure of the plant community, and potentially substrate quality and composition all were related to T. stoloniferum success.

Because T. stoloniferum success varies at both the subcompartment (or watershed) level and at the patch level, I developed two complementary studies to address the questions put forth by Dr. Schuler. In order to guide and refine my studies, I developed three principal hypotheses, which were tested by the two studies I conducted and guided their design:

1) The success of T. stoloniferum is strongly influenced by disturbance history, but disturbance history will interact with environmental variables, including aspect and soil type, to influence population growth and reproductive success.

2) The presence and abundance of $T$. stoloniferum will be strongly correlated with certain properties of the environment, particularly the quantity and quality of light penetrating to the herbaceous layer.

3) A) Trifolium stoloniferum is found associated with some plant species of the regional flora more commonly than others, indicating similar or overlapping physiological requirements. 
B) Physical properties of the understory vegetation, including total percent cover and percent cover by certain plant types (e.g., invasive species) will strongly influence $T$. stoloniferum population size and reproductive success.

A study examining the response of $T$. stoloniferum at the level of the subcompartment or watershed is presented in Chapter II. The purpose of this study was to examine the interacting factors of disturbance history in the form of timber harvesting, aspect, and site quality and their relationship to T. stoloniferum presence and density within subcompartments.

A study examining variation in success of $T$. stoloniferum at the patch level is presented in Chapter III. This study involved detailed habitat assessment of $T$. stoloniferum occurrences of varying abundance and flowering success. Detailed habitat assessment consisted of measurements of light, associated vegetation, substrate, physiographic position, and estimation of time since last disturbance. The relationship of these environmental variables to $T$. stoloniferum abundance and flowering success was assessed using a variety of statistical techniques.

The synthesis of my findings and the implications of my results for management of this species are presented in Chapter IV. 


\section{Literature cited}

Bartgis RL (1985) Rediscovery of Trifolium stoloniferum Muhl. ex A. Eaton. Rhodora 87(851):425-430.

Brooks RE (1983) Trifolium stoloniferum, running buffalo clover: Description, distribution, and current status. Rhodora 85(842):343-354.

Campbell JJN, Evans M, Medley ME \& NL Taylor (1988) Buffalo clovers in Kentucky (Trifolium stoloniferum and T. reflexum): Historical records, presettlement environment, endangered status, cultivation and chromosome number. Rhodora 90(864):399-418.

Chesson P (2000) Mechanisms of maintenance of species diversity. Annual Review of Ecology and Systematics 31:343-366.

Connell JH (1978) Diversity in tropical rain forest and coral reefs. Science 199:1302-1310.

Crawford DJ, Esselman EJ, Windus JL \& CS Pabin (1998) Genetic variation in running buffalo clover (Trifolium stoloniferum: Fabaceae) using random amplified polymorphic DNA markers (RAPDs). Annals of the Missouri Botanical Garden 85(1):81-89.

Cusick AW (1989) Trifolium stoloniferum (Fabaceae) in Ohio: History, habitats, decline and rediscovery. SIDA 13(4): 467-480.

Davis WH (1987) On the decline of Trifolium stoloniferum. Kentucky Native Plant Society Newsletter 2:19.

Denslow JS (1980) Patterns of plant species diversity during succession under different disturbance regimes. Oecologia 46(1):18-21.

Ellison NW, Liston A, Steiner J, Williams WM \& NL Taylor (2006) Molecular phylogenetics of the clover genus (Trifolium- Leguminosae). Molecular Phylogenetics and Evolution 39:688-705.

Ford WM, Madarish D \& TM Schuler (2003) Influence of white-tailed deer digestion on running buffalo clover (Trifolium stoloniferum: Fabaceae Muhl. ex. A. Eaton). American Midland Naturalist 149:425-428.

Franklin CJ (1998) Self-compatibility and variation in seed production among Ohio populations of federally endangered Trifolium stoloniferum (Fabaceae). M.S. Thesis. The Ohio State University, Columbus, OH. xi, 59 leaves : ill.

Grime JP (1973) Competitive exclusion in herbaceous vegetation. Nature 242(5396):344-347.

Hattenbach MJ (1996) Edaphic relations of an endangered plant, Trifolium stoloniferum 
Muhl. ex. A. Eaton. M.S. Thesis. The Ohio State University, Columbus, OH. xii, 74 p. :ill., maps

Hickey RJ, Vincent MA \& SI Guttman (1991) Genetic variation in running buffalo clover (Trifolium stoloniferum, Fabaceae). Conservation Biology 5(3): 309-316.

Homoya MA, Aldrich JR \& EM Jacquart. (1989) The rediscovery of the globally endangered clover, Trifolium stoloniferum, in Indiana. Rhodora 91(866): 207-212.

Huenneke LF \& RJ Hobbs (1992) Disturbance, diversity, and invasion: Implications for conservation. Conservation Biology 6(3):324-337.

Hutchinson GE (1953) The Concept of Pattern in Ecology. Proceedings of the Academy of Natural Sciences of Philadelphia. 105:1-12.

Jakle JA (1968) The American bison and the human occupance of the Ohio Valley. Proceedings of the American Philosophical Society. 112(4):299-305.

Madarish D \& TM Schuler (2002) Effects of forest management practices on the federally endangered running buffalo clover (Trifolium stoloniferum Muhl. ex. A. Eaton). Natural Areas Journal 22(2):120-128.

Madarish DM, Rodrigue JL \& MB Adams (2002) Vascular flora and macroscopic fauna on the Fernow Experimental Forest. USDA Forest Service Northeastern Research Station General Technical Report NE-291. 37pp.

McIntyre S, Díaz S, Lavorel S \& W Cramer (1999) Plant functional types and disturbance dynamics- Introduction. Journal of Vegetation Science 10(5):603-608.

Morris DR, Baligar VC, Schuler TM \& PJ Harmon (2002) Biological nitrogen fixation and habitat of running buffalo clover. Journal of Plant Nutrition 25(4): 735-746.

Nowacki GJ \& MD Abrams (2008) The demise of fire and mesophication of forests in the eastern United States. Bioscience 58(2):123-138.

Quesenberry KH, Call NM, DE Moon \& RA Dunn (1997) Response of native eastern North American Trifolium spp. to root-knot nematodes. Crop Science 37: 270-274.

Roxburgh SH, Shea K \& JB Wilson (2004) The intermediate disturbance hypothesis: Patch dynamics and mechanisms of species coexistence. Ecology 85(2):359-371.

Schuler TM (2004) Fifty years of partial harvesting in a mixed mesophytic forest: composition and productivity. Canadian Journal of Forest Research 34:985-997.

Sehgal OP \& L Payne (1995) Viruses affecting running buffalo clover, Trifolium stoloniferum. Plant Disease 79(3):320. 
Smith LS (1989) Bison in pioneer West Virginia. Prepared for the West Virginia Department of Natural Resources, Natural Heritage Program, Elkins, WV. 27 pp.

United States Fish and Wildlife Service (1987) Endangered and threatened wildlife and plants: determination of endangered status for Trifolium stoloniferum (running buffalo clover). Federal Register 52(108): 21478-21480.

United States Fish and Wildlife Service (1989) Trifolium stoloniferum Recovery Plan. U.S. Fish and Wildlife Service, Twin Cities, MN. 26pp.

United States Fish and Wildlife Service (2007) Running buffalo clover (Trifolium stoloniferum) Recovery Plan: First revision. U. S. Fish \& Wildlife Service, Fort Snelling, MN.

van der Maarel E (1993) Some remarks on disturbance and its relations to diversity and stability. Journal of Vegetation Science 4:733-736. 
Tables

Table 1. Herbs found in association with Trifolium stoloniferum by the mean $\%$ cover of that species measured in habitat assessment plots.

\begin{tabular}{|c|c|c|c|c|c|}
\hline Species name & $\begin{array}{l}\text { Mean \% } \\
\text { cover }\end{array}$ & Species name & $\begin{array}{l}\text { Mean } \% \\
\text { cover }\end{array}$ & Species name & $\begin{array}{c}\text { Mean } \% \\
\text { cover }\end{array}$ \\
\hline $\begin{array}{l}\text { Laportea canadensis (L.) } \\
\text { Weddell }\end{array}$ & 7.85 & Galium triflorum Michx. & 0.44 & Vitis aestivalis Michx. (seedling) & 0.14 \\
\hline Poaceae (various species) & 5.44 & $\begin{array}{l}\text { Hypericum punctatum } \\
\text { Lam. }\end{array}$ & 0.43 & Arctium minus Bernh. & 0.14 \\
\hline $\begin{array}{l}\text { Ageratina altissima var. } \\
\text { altissima (L.) King \& H. Rob. }\end{array}$ & 4.13 & $\begin{array}{l}\text { Symphyotrichum } \\
\text { lateriflorum (L.) A. Löve } \\
\text { \& D. Löve }\end{array}$ & 0.39 & Solidago sp. & 0.14 \\
\hline Viola spp. & 3.38 & Prunella vulgaris L. & 0.38 & Betula lenta L. (seedling) & 0.13 \\
\hline $\begin{array}{l}\text { Symphyotrichum } \\
\text { prenanthoides (Muhl. ex } \\
\text { Willd.) G.L. Nesom }\end{array}$ & 2.35 & Bidens frondosa $\mathrm{L}$. & 0.36 & Prunus serotina Ehrh. (seedling) & 0.12 \\
\hline Rubus allegheniensisPorter & 2.12 & $\begin{array}{l}\text { Polystichum acrostichoides } \\
\text { (Michx.) Schott }\end{array}$ & 0.35 & Lobelia siphilitica L. & 0.12 \\
\hline $\begin{array}{l}\text { Cryptotaenia canadensis (L.) } \\
\text { DC. }\end{array}$ & 1.91 & Verbena urticifolia L. & 0.35 & Packera aurea L. & 0.11 \\
\hline $\begin{array}{l}\text { Trifolium stoloniferum Muhl. } \\
\text { ex Eaton }\end{array}$ & 1.91 & $\begin{array}{l}\text { Liriodendron tulipifera L. } \\
\text { (seedling) }\end{array}$ & 0.34 & Solidago caesia L. & 0.11 \\
\hline Carex spp. & 1.80 & $\begin{array}{l}\text { Eurybia divaricata (L.) } \\
\text { G.L. Nesom }\end{array}$ & 0.32 & Monarda clinopodia L. & 0.11 \\
\hline $\begin{array}{l}\text { Amphicarpaea bracteata (L.) } \\
\text { Fernald }\end{array}$ & 1.75 & Polygonum persicaria L. & 0.28 & Hydrophyllum virginianum L. & 0.10 \\
\hline Collinsonia canadensis L. & 1.62 & $\begin{array}{l}\text { Unidentified opposite- } \\
\text { leaved herb }\end{array}$ & 0.27 & Sanicula canadensis L. & 0.10 \\
\hline
\end{tabular}




\begin{tabular}{|c|c|c|c|c|c|}
\hline $\begin{array}{l}\text { Microstegium vimineum } \\
\text { (Trin.) A. Camus }\end{array}$ & 1.36 & Rumex obtusifolius L. & 0.27 & Campanulastrum americanum L. & 0.10 \\
\hline Impatiens sp. & 1.33 & $\begin{array}{l}\text { Dryopteris intermedia } \\
\text { (Muhl. ex Willd.) }\end{array}$ & 0.27 & Uvularia grandiflora Sm. & 0.09 \\
\hline $\begin{array}{l}\text { Deparia acrostichoides (Sw.) } \\
\text { M. Kato }\end{array}$ & 1.25 & $\begin{array}{l}\text { Osmorhiza longistylis } \\
\text { (Torr.) DC }\end{array}$ & 0.25 & Arisaema triphyllum L. & 0.09 \\
\hline Polygonum virginianum L. & 1.13 & Smilax rotundifolia L. & 0.25 & Solidago flexicaulis L & 0.08 \\
\hline $\begin{array}{l}\text { Symphyotrichum cordifolium } \\
\text { (L.) G.L. Nesom }\end{array}$ & 0.93 & Helianthus decapetalus L. & 0.24 & $\begin{array}{l}\text { Sambucus nigra L. ssp. } \\
\text { canadensis (L.) R. Bolli } \\
\text { (seedling) }\end{array}$ & 0.08 \\
\hline Potentilla simplex Michx. & 0.88 & Rubus occidentalis L. & 0.22 & Lactuca serriola L. & 0.08 \\
\hline Oxalis stricta $\mathrm{L}$ & 0.79 & Scutellaria lateriflora L. & 0.21 & Ulmus rubra L. & 0.08 \\
\hline $\begin{array}{l}\text { Fraxinus americana L. } \\
\text { (seedling) }\end{array}$ & 0.78 & $\begin{array}{l}\text { Aristolochia macrophylla } \\
\text { Lam. }\end{array}$ & 0.21 & $\begin{array}{l}\text { Acer pensylvanicum L. } \\
\text { (seedling) }\end{array}$ & 0.07 \\
\hline Geum canadense Jacq. & 0.75 & $\begin{array}{l}\text { Thelypteris noveboracensis } \\
\text { L. Nieuwl. }\end{array}$ & 0.20 & $\begin{array}{l}\text { Dennstaedtia punctilobula } \\
\text { (Michx.) T. Moore }\end{array}$ & 0.07 \\
\hline $\begin{array}{l}\text { Actaea racemosa L. var. } \\
\text { racemosa }\end{array}$ & 0.68 & Aster sp. & 0.19 & Eupatorium purpureum L. & 0.06 \\
\hline Hydrophyllum canadense L. & 0.67 & Tussilago farfara $\mathrm{L}$. & 0.19 & Polygonum sagittatum $L$. & 0.06 \\
\hline Pilea pumila (L.) A. Gray & 0.65 & Asarum canadense L. & 0.18 & Heuchera americana L. & 0.06 \\
\hline Sedum ternatum Michx. & 0.57 & $\begin{array}{l}\text { Quercus rubra L. } \\
\text { (seedling) }\end{array}$ & 0.17 & Prenanthes alba L. & 0.06 \\
\hline $\begin{array}{l}\text { Verbesina alternifolia (L.) } \\
\text { Britton ex Kearney }\end{array}$ & 0.51 & Asclepias exaltata L. & 0.17 & Galinsoga quadriradiata Cav. & 0.06 \\
\hline Circaea lutetiana L. & 0.49 & Thalictrum dioicum L. & 0.16 & Monarda didyma L. & 0.06 \\
\hline Sanicula sp. & 0.46 & $\begin{array}{l}\text { Phegopteris hexagonoptera } \\
\text { (Michx.) Fée }\end{array}$ & 0.16 & Onoclea sensibilis L. & 0.06 \\
\hline Erigeron annuus (L.) Pers. & 0.46 & Carex plantaginea Lam. & 0.15 & Sanicula trifoliata E.P. Bicknell & 0.05 \\
\hline $\begin{array}{l}\text { Acer saccharum Marsh. } \\
\text { (seedling) }\end{array}$ & 0.44 & $\begin{array}{l}\text { Dicanthelium clandestinum } \\
\text { (L.) Gould }\end{array}$ & 0.14 & & \\
\hline Plantago rugellii Decne. & 0.44 & & & & \\
\hline
\end{tabular}


Table 2. Shrub cover by species and the mean distance that each shrub species intersect with the transect in habitat assessment plots.

\begin{tabular}{|l|c|l|c|}
\hline Species name & $\begin{array}{l}\text { Mean distance (m) } \\
\text { per plot }\end{array}$ & Species name & $\begin{array}{l}\text { Mean distance (m) } \\
\text { per plot }\end{array}$ \\
\hline $\begin{array}{l}\text { Rubus allegheniensis } \\
\text { Porter }\end{array}$ & 1.47 & Crataegus sp. & 0.03 \\
\hline Vitis aestivalis Michx. & 0.43 & $\begin{array}{l}\text { Hydrangea arborescens } \\
\text { L. }\end{array}$ & 0.03 \\
\hline $\begin{array}{l}\text { Sambucus nigra L. ssp. } \\
\text { canadensis (L.) R. Bolli }\end{array}$ & 0.30 & Aralia spinosa L. & 0.02 \\
\hline Smilax rotundifolia L. & 0.15 & Cornus alternifolia L. f. & 0.01 \\
\hline Rhus typhina L. & 0.09 & Rubus odoratus L. & 0.01 \\
\hline $\begin{array}{l}\text { Lindera benzoin (L.) } \\
\text { Blume }\end{array}$ & 0.08 & $\begin{array}{l}\text { Aristolochia } \\
\text { macrophylla Lam. }\end{array}$ & 0.00 \\
\hline & & $\begin{array}{l}\text { Sambucus racemosa L. } \\
\text { var. racemosa }\end{array}$ & \\
Hamamelis virginiana L. & 0.06 & & 0.00 \\
\hline Rubus occidentalis L. & 0.04 & & \\
\hline
\end{tabular}


Table 3. Mean basal area of trees in $\mathrm{ft}^{2} / \mathrm{ac}$ as measured during the habitat assessment process for T. stoloniferum.

\begin{tabular}{|l|c|l|c|}
\hline Species name & $\begin{array}{l}\text { Mean BA per } \\
\text { plot }\left(\mathrm{ft}^{2} / \mathrm{ac}\right)\end{array}$ & Species name & $\begin{array}{l}\text { Mean BA per } \\
\text { plot }\left(\mathrm{ft}^{2} / \mathrm{ac}\right)\end{array}$ \\
\hline Acer saccharum Marsh. & 30.48 & Carya ovata (Mill.) K. Koch & 1.43 \\
\hline Liriodendron tulipifera L. & 27.86 & Acer rubrum L. & 0.71 \\
\hline Quercus rubra. L. & 11.67 & Ulmus rubra Muhl. & 0.71 \\
\hline Prunus serotina Ehrh. & 9.05 & Carya cordiformis (Wangenh.) K. Koch & 0.48 \\
\hline Fraxinus americana L. & 3.33 & Ostrya virginiana (Mill.) K. Koch & 0.48 \\
\hline Robinia pseudoacacia L. & 3.33 & Carya glabra (Mill.) Sweet & 0.24 \\
\hline Fagus grandifolia Ehrh. & 3.10 & Magnolia acuminata (L.) L. & 0.24 \\
\hline Tilia americana L. & 2.38 & Nyssa sylvativa Marsh. & 0.24 \\
\hline Betula lenta L. & 1.43 & Quercus alba L. & 0.24 \\
\hline
\end{tabular}

Table 4. Mean number of saplings (trees $<10 \mathrm{~cm}$ in diameter at breast height) found inside the $100 \mathrm{~m}^{2}$ habitat assessment plots.

\begin{tabular}{|l|l|l|c|}
\hline Species name & $\begin{array}{l}\text { Mean \# of saplings } \\
\text { per plot }\end{array}$ & Species name & $\begin{array}{l}\text { Mean \# of } \\
\text { saplings per plot }\end{array}$ \\
\hline Liriodendron tulipifera L. & 3.40 & Ulmus rubra Muhl. & 0.21 \\
\hline Acer saccharum Marsh. & 3.38 & Magnolia acuminata (L.) L. & 0.14 \\
\hline Betula lenta L. & 3.36 & Carya cordiformis (Wangenh.) K. Koch & 0.10 \\
\hline Fagus grandifolia Ehrh. & 1.86 & Quercus rubra. L. & 0.07 \\
\hline Ostrya virginiana (Mill.) K. Koch & 1.24 & Cornus alternifolia L. f. & 0.05 \\
\hline Fraxinus americana L. & 1.07 & Rhus typhina L. & 0.05 \\
\hline Robinia pseudoacacia L. & 0.88 & Aralia spinosa L. & 0.02 \\
\hline Acer pensylvanicum L. & 0.67 & Carya ovata (Mill.) K. Koch & 0.02 \\
\hline Prunus serotina Ehrh. & 0.36 & Juglans cinerea L. & 0.02 \\
\hline Tilia americana L. & 0.36 & Platanus occidentalis L. & 0.02 \\
\hline
\end{tabular}


Burkhart 24

\section{Figures}



Figure 1. Photograph of Trifolium stoloniferum. Depicts both a small flowering head and ripening fruits. The paired leaves located along the inflorescence stalk approximately 1-3 inches below the inflorescence clearly distinguish this species from T. repens 


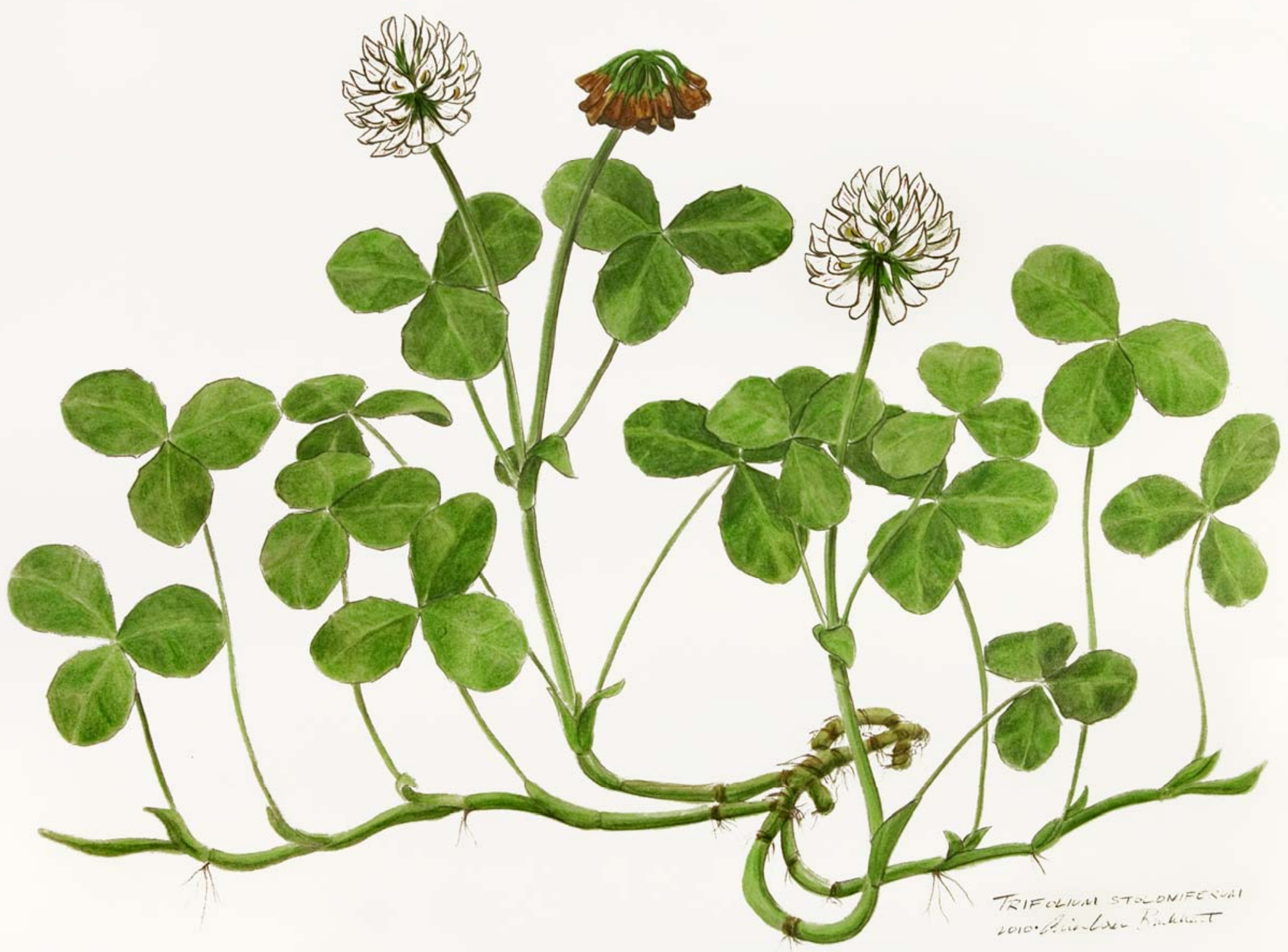

Figure 2a. Illustration of Trifolium stoloniferum. Identifying characteristics depicted are paired leaves below the inflorescence, stoloniferous habit with rooting at nodes, lack of prominent white stripe in center of leaflet, and slightly toothed leaflet margins. 
Burkhart 26

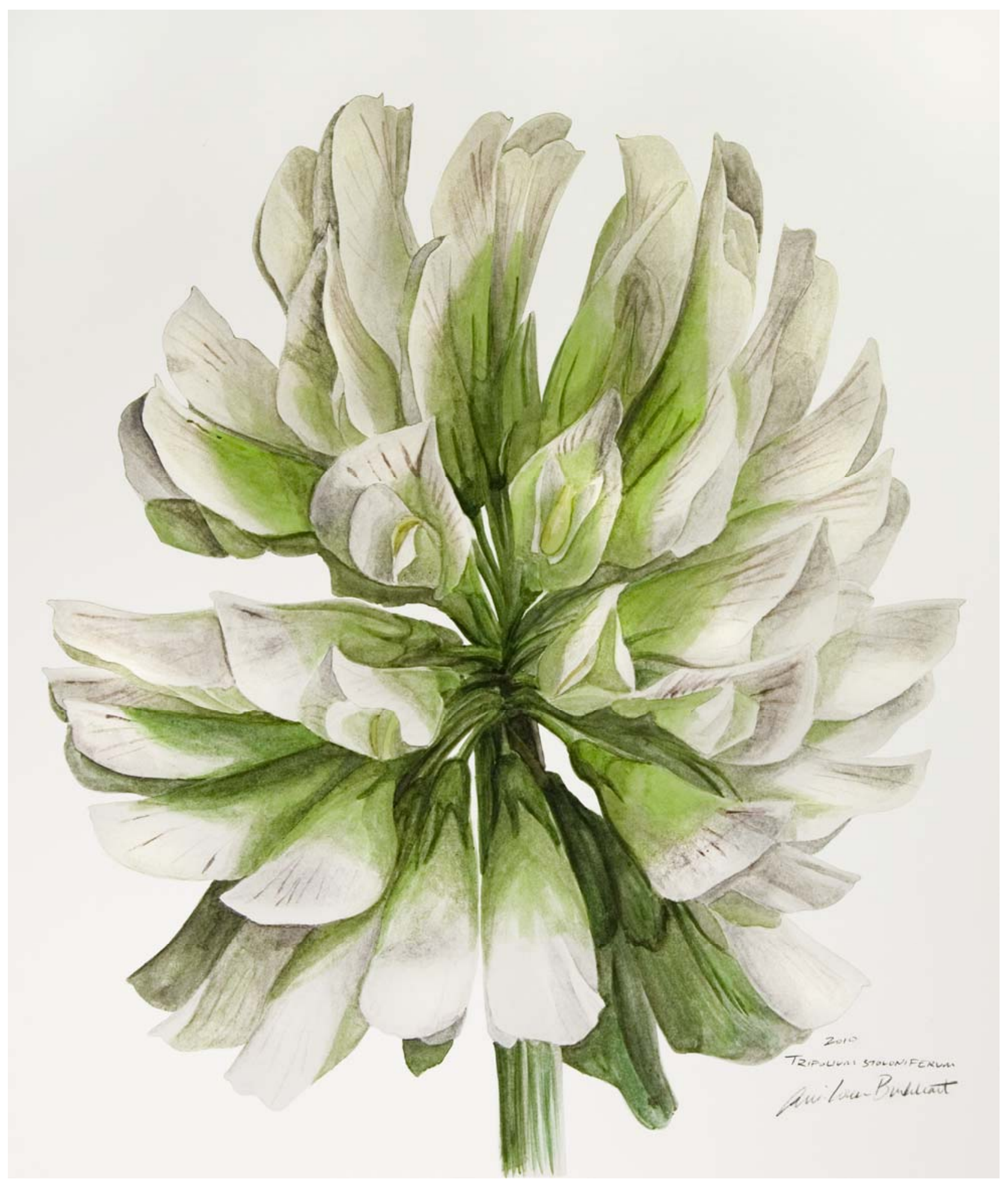

Figure 2b. Close-up of T. stoloniferum inflorescence. 




Figure 3. Extant distribution of Trifolium stoloniferum. The largest occurrences are located in the mountain regions of West Virginia (largest populations $\sim 35,000$ ), while the greatest number of individual occurrences are found in and around the Bluegrass Army Depot in Kentucky. 
Burkhart 28

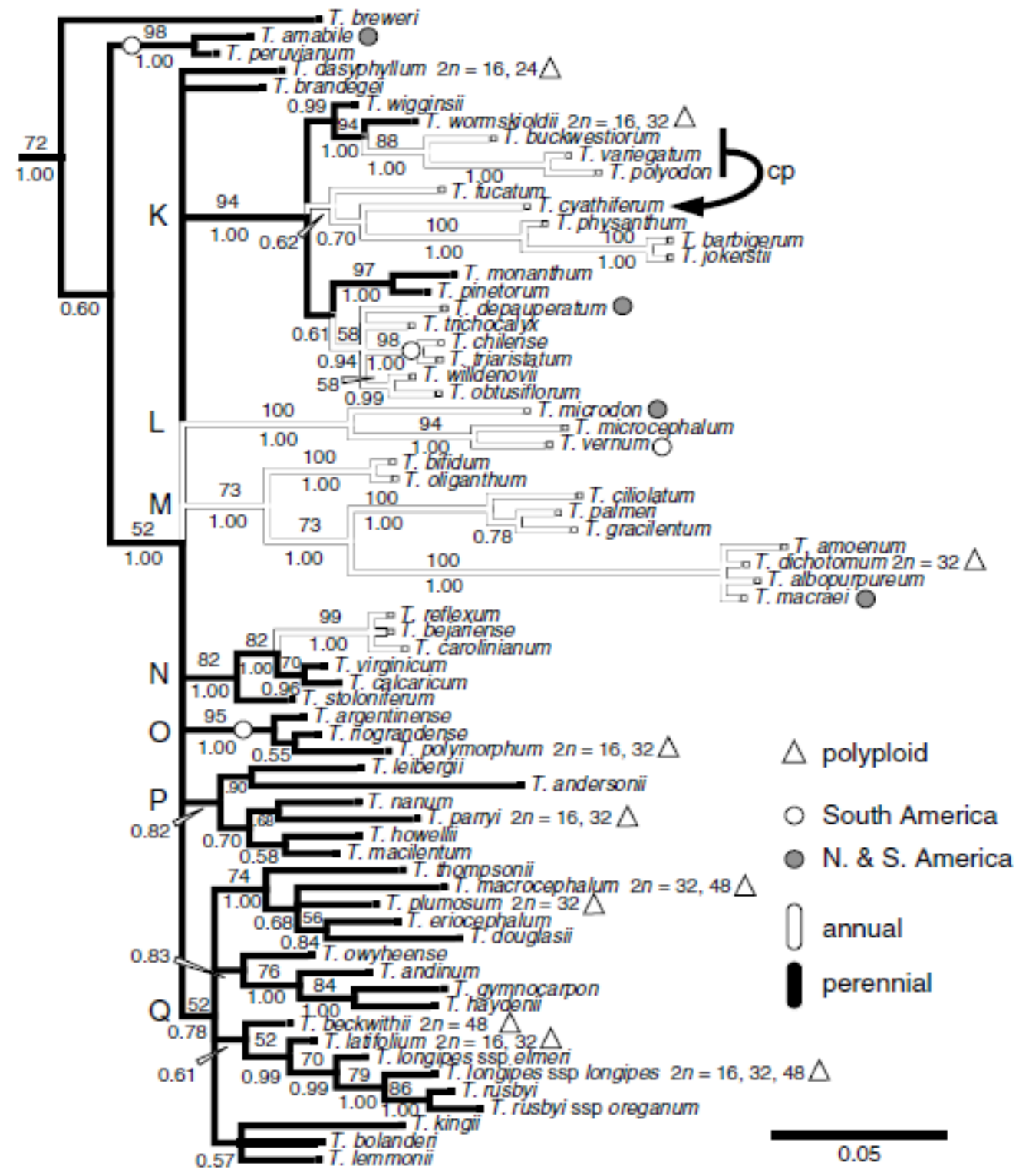

Figure 4. Phylogenetic tree of the genus Trifolium. Trifolium stoloniferum is the basal member of a small clade of clovers (6 species) native to eastern North America (Reprinted with permission from Ellison et al. 2006). 


\section{Appendix}

Appendix 1. Geographic names using "Buffalo" in West Virginia and parts of surrounding states. These place names were gathered from the USGS website $<$ http://geonames.usgs.gov $>$ and entering the search term "buffalo" and selecting WV as the state. I excluded schools, reservoirs, fire towers, and other feature types that derive their name from the place in which they are situated. Here is a listing of streams, valleys, populated places and locales found in WV or surrounding states that include the word "buffalo" in their name.

\begin{tabular}{|c|c|c|c|c|c|c|c|}
\hline Name & Feature type & County & State & Latitude & Longitutude & Elevation & Map \\
\hline Buffalo Gap Spring & Spring & Hampshire & WV & $392000 \mathrm{~N}$ & $0782724 \mathrm{~W}$ & 958 & Capon Bridge \\
\hline Buffalo Branch & Stream & Putnam & WV & $383635 \mathrm{~N}$ & $0815241 \mathrm{~W}$ & 581 & Winfield \\
\hline Buffalo Calf Fork & Stream & Doddridge & WV & $391528 \mathrm{~N}$ & $0803858 \mathrm{~W}$ & 860 & Smithburg \\
\hline Buffalo Creek & Stream & Wyoming & WV & $373226 \mathrm{~N}$ & $0814642 \mathrm{~W}$ & 1102 & Gilbert \\
\hline Buffalo Creek & Stream & Lincoln & WV & $381944 \mathrm{~N}$ & $0820737 \mathrm{~W}$ & 597 & West Hamlin \\
\hline Buffalo Creek & Stream & Harrison & WV & $391204 \mathrm{~N}$ & $0802448 \mathrm{~W}$ & 968 & West Milford \\
\hline Buffalo Creek & Stream & Summers & WV & $373226 \mathrm{~N}$ & $0805302 \mathrm{~W}$ & 1421 & Pipestem \\
\hline Buffalo Creek & Stream & Mingo & WV & $374158 \mathrm{~N}$ & $0821748 \mathrm{~W}$ & 640 & Williamson \\
\hline Buffalo Creek & Stream & Logan & WV & $374429 \mathrm{~N}$ & $0815239 \mathrm{~W}$ & 738 & Man \\
\hline Buffalo Creek & Stream & Greenbrier & WV & $375125 \mathrm{~N}$ & $0804253 \mathrm{~W}$ & 2421 & Dawson \\
\hline Buffalo Creek & Stream & Logan & WV & $375345 \mathrm{~N}$ & $0815931 \mathrm{~W}$ & 633 & Henlawson \\
\hline Buffalo Creek & Stream & Fayette & WV & $375440 \mathrm{~N}$ & $0810131 \mathrm{~W}$ & 1076 & Thurmond \\
\hline Buffalo Creek & Stream & Wayne & WV & $382116 \mathrm{~N}$ & $0823030 \mathrm{~W}$ & 518 & Burnaugh \\
\hline Buffalo Creek & Stream & Clay & WV & $382741 \mathrm{~N}$ & $0810422 \mathrm{~W}$ & 679 & Clay \\
\hline Buffalo Creek & Stream & Putnam & WV & $383245 \mathrm{~N}$ & $0815719 \mathrm{~W}$ & 541 & Winfield \\
\hline Buffalo Creek & Stream & Braxton & WV & $383922 \mathrm{~N}$ & $0804435 \mathrm{~W}$ & 804 & Sutton \\
\hline Buffalo Creek & Stream & Roane & WV & $384927 \mathrm{~N}$ & 0813206W & 669 & Gay \\
\hline Buffalo Creek & Stream & Braxton & WV & $385157 \mathrm{~N}$ & $0804030 \mathrm{~W}$ & 741 & Burnsville \\
\hline Buffalo Creek & Stream & Grant & WV & $391628 \mathrm{~N}$ & 0792152W & 2333 & Gorman \\
\hline Buffalo Creek & Stream & Hampshire & WV & $392232 \mathrm{~N}$ & $0784425 \mathrm{~W}$ & 640 & Springfield \\
\hline Buffalo Creek & Stream & Marion & WV & $392944 \mathrm{~N}$ & $0800730 \mathrm{~W}$ & 860 & Fairmont West \\
\hline Buffalo Creek & Stream & Brooke & WV & $401552 \mathrm{~N}$ & $0803655 \mathrm{~W}$ & 646 & Steubenville \\
\hline
\end{tabular}




\begin{tabular}{|c|c|c|c|c|c|c|c|}
\hline & & & & & & & East \\
\hline Buffalo Fork & Stream & Raleigh & WV & $375434 \mathrm{~N}$ & $0812013 \mathrm{~W}$ & 1503 & Pax \\
\hline Buffalo Run & Stream & Jackson & WV & $390329 \mathrm{~N}$ & $0813726 \mathrm{~W}$ & 643 & Rockport \\
\hline Buffalo Run & Stream & Ritchie & WV & $390801 \mathrm{~N}$ & $0811640 \mathrm{~W}$ & 620 & Petroleum \\
\hline Buffalo Run & Stream & Preston & WV & $392734 \mathrm{~N}$ & $0793922 \mathrm{~W}$ & 1224 & Kingwood \\
\hline Buffalo Run & Stream & Webster & WV & $384338 \mathrm{~N}$ & $0802335 \mathrm{~W}$ & 1263 & Hacker Valley \\
\hline Buffalo Run & Stream & Hampshire & WV & $391549 \mathrm{~N}$ & $0784926 \mathrm{~W}$ & 686 & Romney \\
\hline Buffalo Run & Stream & Wetzel & WV & $393152 \mathrm{~N}$ & $0803832 \mathrm{~W}$ & 738 & Pine Grove \\
\hline Buffalo Run & Stream & Preston & WV & $394129 \mathrm{~N}$ & 0792416W & 1437 & Friendsville \\
\hline Buffalolick Branch & Stream & Kanawha & WV & $382631 \mathrm{~N}$ & $0812806 \mathrm{~W}$ & 581 & Blue Creek \\
\hline Buffalolick Run & Stream & Jackson & WV & $384534 \mathrm{~N}$ & $0813858 \mathrm{~W}$ & 620 & Ripley \\
\hline Buffalolick Run & Stream & Roane & WV & $384354 \mathrm{~N}$ & $0812237 \mathrm{~W}$ & 791 & Walton \\
\hline Bufflick Fork & Stream & Kanawha & WV & $381256 \mathrm{~N}$ & $0812117 \mathrm{~W}$ & 669 & Montgomery \\
\hline Little Buffalo Creek & Stream & Lincoln & WV & $381959 \mathrm{~N}$ & $0820756 \mathrm{~W}$ & 597 & West Hamlin \\
\hline Little Buffalo Creek & Stream & Putnam & WV & $383406 \mathrm{~N}$ & $0815851 \mathrm{~W}$ & 541 & Winfield \\
\hline Little Buffalo Creek & Stream & Braxton & WV & $383921 \mathrm{~N}$ & $0804543 \mathrm{~W}$ & 804 & Gassaway \\
\hline Little Buffalo Creek & Stream & Grant & WV & $391545 \mathrm{~N}$ & $0792145 \mathrm{~W}$ & 2428 & Gorman \\
\hline Middle Fork Buffalo Creek & Stream & Logan & WV & $374755 \mathrm{~N}$ & $0814001 \mathrm{~W}$ & 1509 & Lorado \\
\hline North Fork Buffalo Creek & Stream & Logan & WV & $374811 \mathrm{~N}$ & $0813912 \mathrm{~W}$ & 1585 & Lorado \\
\hline Right Fork Buffalo Creek & Stream & Logan & WV & $375336 \mathrm{~N}$ & $0820030 \mathrm{~W}$ & 696 & Chapmanville \\
\hline Right Fork Buffalo Creek & Stream & Logan & WV & $374605 \mathrm{~N}$ & $0815017 \mathrm{~W}$ & 846 & Amherstdale \\
\hline South Fork Buffalo Creek & Stream & Mingo & WV & $374214 \mathrm{~N}$ & $0821613 \mathrm{~W}$ & 699 & Williamson \\
\hline Buffalo Creek & Stream & Preston & WV & $391915 \mathrm{~N}$ & $0794112 \mathrm{~W}$ & 1398 & Rowlesburg \\
\hline Buffalo Run & Stream & Pocahontas & WV & $382742 \mathrm{~N}$ & $0794845 \mathrm{~W}$ & 2618 & Green Bank \\
\hline Buffalo Run & Stream & Pendleton & WV & $384258 \mathrm{~N}$ & 0791907W & 1591 & Franklin \\
\hline Buffalo Run & Stream & Randolph & WV & $385550 \mathrm{~N}$ & $0795028 \mathrm{~W}$ & 1942 & Elkins \\
\hline Buffalo Run & Stream & Tyler & WV & $392921 \mathrm{~N}$ & $0805902 \mathrm{~W}$ & 653 & Middlebourne \\
\hline Buffalo Run & Stream & Tyler & WV & $392819 \mathrm{~N}$ & $0810103 \mathrm{~W}$ & 633 & Bens Run \\
\hline Buffalo Fork & Stream & Pocahontas & WV & $383228 \mathrm{~N}$ & 0794326W & 2943 & Thornwood \\
\hline Left Buffalo Run & Stream & Tyler & WV & $393019 \mathrm{~N}$ & $0805918 \mathrm{~W}$ & 696 & Paden City \\
\hline Little Buffalo Creek & Stream & Preston & WV & $391912 \mathrm{~N}$ & $0794056 \mathrm{~W}$ & 1398 & Rowlesburg \\
\hline Little Buffalo Creek & Stream & Preston & WV & $391641 \mathrm{~N}$ & 0794424W & 1768 & Rowlesburg \\
\hline
\end{tabular}




\begin{tabular}{|c|c|c|c|c|c|c|c|}
\hline Little Buffalo Run & Stream & Tyler & WV & $393009 \mathrm{~N}$ & 0810058W & 725 & $\begin{array}{l}\text { New } \\
\text { Matamoras }\end{array}$ \\
\hline Right Buffalo Run & Stream & Tyler & WV & $393019 \mathrm{~N}$ & $0805918 \mathrm{~W}$ & 696 & Paden City \\
\hline Buffalo Mountain & Summit & Greenbrier & WV & $375823 \mathrm{~N}$ & $0803324 \mathrm{~W}$ & 3950 & Cornstalk \\
\hline Buffalo Mountain & Summit & Logan & WV & $374611 \mathrm{~N}$ & $0814149 \mathrm{~W}$ & 2457 & Lorado \\
\hline Buffalo Hills & Summit & Pendleton & WV & $384157 \mathrm{~N}$ & $0792021 \mathrm{~W}$ & 2402 & Franklin \\
\hline Buffalo Knob & Summit & Summers & WV & $374700 \mathrm{~N}$ & 0805026W & 3015 & $\begin{array}{l}\text { Meadow } \\
\text { Bridge }\end{array}$ \\
\hline Buffalo Mountain & Summit & Mingo & WV & $374217 \mathrm{~N}$ & $0821250 \mathrm{~W}$ & 1896 & Delbarton \\
\hline Buffalo Bull Knob & Summit & Webster & WV & $383053 \mathrm{~N}$ & $0802316 \mathrm{~W}$ & 2799 & Diana \\
\hline Buffalo Hollow & Valley & Wirt & WV & $385924 \mathrm{~N}$ & $0812331 \mathrm{~W}$ & 633 & Reedy \\
\hline$\overline{\text { Buffalo Gap Camp }}$ & Locale & Hampshire & WV & $392006 \mathrm{~N}$ & $0782731 \mathrm{~W}$ & 1033 & Capon Bridge \\
\hline Camp Buffalo (historical) & Locale & Putnam & WV & $383705 \mathrm{~N}$ & $0815847 \mathrm{~W}$ & 574 & Winfield \\
\hline Buffalo & Populated Place & Putnam & WV & $383703 \mathrm{~N}$ & $0815854 \mathrm{~W}$ & 568 & Winfield \\
\hline$\overline{\text { Buffalo Creek }}$ & Populated Place & Wayne & WV & $382120 \mathrm{~N}$ & $0823034 \mathrm{~W}$ & 548 & Burnaugh \\
\hline Buffalo (historical) & Populated Place & Jackson & WV & $384938 \mathrm{~N}$ & $0813202 \mathrm{~W}$ & 676 & Gay \\
\hline Buffalo Station (historical) & Populated Place & Marion & WV & $392945 \mathrm{~N}$ & $0800735 \mathrm{~W}$ & 879 & Fairmont West \\
\hline Buffalolick (historical) & Populated Place & Roane & WV & $384215 \mathrm{~N}$ & $0812050 \mathrm{~W}$ & 853 & Looneyville \\
\hline
\end{tabular}




\title{
Chapter II
}

\section{Effects of forest management on running buffalo clover (Trifolium stoloniferum) distribution and abundance in the Fernow Experimental Forest}

\begin{abstract}
Trifolium stoloniferum, or running buffalo clover, is a federally endangered plant species that occurs on the Fernow Experimental Forest in Tucker County, West Virginia, at the time of publication of this thesis. Previous work and extensive anecdotal evidence suggests that this species is maintained in part by periodic disturbances to its habitat. In the Fernow Experimental Forest, this disturbance is in the form of intermittent logging activities. I investigated the role of historical forestry practices and several environmental variables in fostering T. stoloniferum at the stand level. Censuses have been conducted on all known T. stoloniferum occurrences in the Fernow since 1994. Occurrences were grouped by subcompartment or watershed, which are the basic units of management activity within this experimental forest. Site characteristics and disturbance history variables were assessed for their impact upon T. stoloniferum presence and density. Classification tree analysis identified the total number of forest harvest events in a subcompartment since 1948 as the most important predictor of T. stoloniferum presence or absence. Regression tree analysis identified subcompartment aspect as important in determining T. stoloniferum abundance, with west-facing compartments supporting larger populations. The findings of this study confirm the importance of disturbance in maintaining healthy populations of running buffalo clover. However, site characteristics independent of disturbance history also are predictors of $T$. stoloniferum presence and abundance, suggesting that managers attempting to restore or create habitat for $T$.
\end{abstract}


stoloniferum should be aware of and plan for the interaction between disturbance history and site characters in determining the suitability of habitat for T. stoloniferum.

\section{Introduction}

The creation of reserves and habitat protection may often be insufficient for the conservation of species and unique communities (Hobbs \& Huenneke 1992). One cause of this failure is a disruption, change, or cessation of the disturbance processes that allowed for the persistence of that species, community, or particular landscape structure (Baker 1994, Simberloff 2004, Brawn et al. 2001). Restoration or approximation of disturbances that maintained these disturbance-dependent species was determined to be missing and found to be necessary to achieve desired population growth and stability (Pickett et al. 1989). Disturbance can maintain diversity in ecological communities by disrupting succession, creating and maintaining habitat heterogeneity, shifting the dynamics of competitive relationships within communities, and facilitating the recruitment of new individuals from outside of and within the community (van der Maarel 1993, Chesson 2000).

Trifolium stoloniferum Muhl. ex A. Eaton was said in 1983 to be one of the rarest members of the North American flora (Brooks 1983). Since that time, populations have been discovered in three regions: the Missouri Ozarks, the Bluegrass region of Kentucky and surrounding states, and the Allegheny Mountains of West Virginia (USFWS 2007). The species has flourished at the Fernow Experimental Forest in Tucker County near Parsons, WV. The Fernow is an experimental forest that was designated for research in 1934. Forest management research and harvest activities were initiated in many stands in the late 1940s and 1950s (Schuler 2004). 
Trifolium stoloniferum is found across its range in seemingly disparate habitatsold cemeteries, lawns of plantation style homes, rich soils next to roads, lightly grazed pastures, and managed mesic forests (USFWS 2007). However, two commonalities that the majority of these sites have are that they are underlain by calcareous bedrock and they experience periodic disruption of the structure of their habitat. The pattern of occurrences of $T$. stoloniferum at this and other locations suggest that the plant is adapted to moderate levels of disturbance to its habitat. Anecdotal evidence and previous studies suggest that regular disturbances such as mowing, light grazing, moderate logging activity, and prescribed burning promote asexual and sexual reproduction of T. stoloniferum (USFWS 2007).

The goal of this study is to quantify the role of forest management activities on the Fernow Experimental Forest in determining the presence and abundance of $T$. stoloniferum. In addition, I will examine the role of environmental characteristics in maintaining or promoting T. stoloniferum and identify interactions between disturbance history and environmental characteristics that affect the establishment, growth, or persistence of the species.

\section{Methods}

The study area was entirely within the Fernow Experimental Forest $\left(39.03^{\circ} \mathrm{N}\right.$, $79.67^{\circ} \mathrm{W}$ ), a research forest established in the Monongahela National Forest in 1934 approximately $2.5 \mathrm{~km}$ south of the town of Parsons, WV, in Tucker County. This forest resides within the Allegheny Mountains Section of the Central Appalachian Broadleaf Forest (McNab \& Avers 1994). Elevations at the Forest range from 533 to 1112 meters above sea level. Steep slopes predominate at the forest, with most of the landscape at 20 
to 30 percent (Madarish et al. 2000). All site aspects are represented, but north, west, and southeast-facing slopes predominate. Bedrock geology is a combination of sandstone, siltstone, shale, and limestone, with several significant karst formations associated with the limestone. The Greenbrier soil series is present in the Fernow Experimental Forest, and a majority of the T. stoloniferum occurrences are found in association with this soil. Mean temperature is $9^{\circ} \mathrm{C}$, and mean precipitation is $145 \mathrm{~cm}$ which is distributed throughout the year. Summers are mild and winters are cold. Forest types at the Fernow are mostly mixed mesophytic hardwood forests, with red-spruce and northern hardwood forests gaining importance in a few areas at higher altitudes and hemlock important along riparian corridors. Understory vegetation is shaped by the interaction between physiographic characters, disturbance regime, and soils and varies from sparsely vegetated to thick with herbaceous vegetation such as stinging nettle (Laportea canadensis L.), wingstem (Verbesina alternifolia L.), and other forest herbs to dense rhododendron thickets (Rhododendron maximum L. and Kalmia latifolia L.).

The study sites included in this study consisted of 39 subcompartments and watersheds located within the Fernow Experimental Forest. After World War II, the United States Forest Service established a research program in the Fernow oriented towards long-term silviculture of central Appalachian hardwood forests. Many of the subcompartments included in this study have been continually managed with the same silvicultural system since the early 1950's. Subcompartments are the fundamental unit of management within the Fernow- silvicultural prescriptions are applied at this level, and forest inventories are conducted at this level. 
Trifolium stoloniferum was first detected at the Fernow in 1993, and systematic efforts to annually census populations have been undertaken since 1998. Newly established or discovered populations were added to census efforts as they were detected. Censuses were total, with efforts being made to find and count all rooted crowns in every known population in the experimental forest. The census methods employed in the Fernow were in accordance with the population monitoring requirements of the U.S. Fish and Wildlife Service Recovery Plan for T. stoloniferum (USFWS 2007).

Using ArcGIS 9.3 (ESRI 2009), I created a map of all known T. stoloniferum occurrences and all subcompartments within the Fernow. All occurrences not within the boundaries of a subcompartment or other management unit with known disturbance history and measured environmental variables were excluded from analysis. For each year that censuses had been conducted, total T. stoloniferum crowns were tallied by subcompartment for every population occurring within its boundaries. Trifolium stoloniferum densities by subcompartment were calculated for every annual census by dividing total number of crowns by the area of the subcompartment in acres. I used the median crowns/acre density in a subcompartment since censuses were initiated in 1998 until the most recent census in 2008 to account for the range of T. stoloniferum population size over time. Median crowns/acre was chosen over mean crowns/acre as a better representation of population size because the median is less sensitive than the mean to extreme variations particularly with a small sample size (Zar 1999). Trifolium stoloniferum populations at the Fernow undergo rapid fluctuations in population size immediately following logging events: I chose median crowns/acre as a better index of the long-term habitat suitability of each subcompartment. 
In order to draw distinctions and inferences about the relative suitability of a compartment as T. stoloniferum habitat, I included in this analysis 23 subcompartments that never contained this species. These 23 subcompartments plus the 16 subcompartments that contained $\mathrm{RBC}$ for at least one year still do not account for all subcompartments in the Fernow. The criteria for selection of $T$. stoloniferum-absent subcompartments was based on either being contiguous to $T$. stoloniferum-present subcompartments or possessing the Greenbrier soil series, a limestone-derived soil with which many of the T. stoloniferum populations of the Allegheny Mountains are associated.

Using median crowns/acre density I constructed a set of four $T$. stoloniferum density categories. Sixteen total subcompartments or watersheds have contained $T$. stoloniferum in at least one census, and populations vary from 0 to 70.6 median crowns/acre (I chose to use acre instead of hectare because research at the Fernow has largely been conducted in English measurements, not SI). These density categories are similar to the population categories described in the recovery plan (USFWS 2007), in which populations are ranked as $\mathrm{A}, \mathrm{B}, \mathrm{C}$, or $\mathrm{D}$ according to their size and long-term prospects for persistence. In the recovery plan, however, the categories refer to populations, and all occurrences within the Fernow Experimental Forest are considered a single population by the authors of the recovery plan. The four categories I used for this analysis were A- absent, B-0.01-10 crowns/acre, C-10.01-35 crowns/acre, and D- greater than 35 crowns/acre. These categories are useful in analysis and application, as researchers and managers are able to identify those environmental and disturbance history variables that promote the growth of large, medium, and small populations or 
densities, all of which are necessary to achieve conservation goals for the species (USFWS 2007). Trifolium stoloniferum presence or absence within a subcompartment was also considered.

Subcompartment disturbance history was determined using forest inventories conducted before and after every harvest that occurred in that subcompartment. The data consisted of total basal area (BA) before harvest, total BA removed during the harvest, and residual BA for every subcompartment. Previous studies and strong anecdotal evidence suggest that $T$. stoloniferum is most often associated with skid roads in stands managed in an uneven-aged silvicultural system, where gaps are created at periodic intervals and the skid roads used to remove logs from the stand are used at intervals of approximately 10 years (Madarish \& Schuler 2002). Using these insights to guide analysis, I constructed four disturbance variables that captured the effects of timber harvest upon stand structure and disturbance state that are relevant to T. stoloniferum: 1) time elapsed since last disturbance, using 2008 as the baseline, i.e., the most recent year in which a census was conducted at the time of this study 2) proportion of basal area removed in the last disturbance 3) total number of disturbances that have occurred since the initiation of management in that stand and 4) total basal area removed per acre from the stand since management began in the stand.

Site characteristic variables were also considered for every subcompartment. The oak site index, an indicator of the fifty-year height growth potential for oak trees (Quercus spp.), was used as an indicator of overall fertility of the site. Oak site index is a common and accepted measure of forest productivity within this region (Lamson 1987). The presence of the Greenbrier soil series, a limestone derived soil series and a strong 
predictor of the presence of $T$. stoloniferum, was included in the analysis. The most important use of this variable is to assess why those subcompartments that contain this soil series, an important predictor of habitat suitability for T. stoloniferum, do not harbor this species. The aspect of the subcompartment was also considered, as aspect is a predictor of the growth potential and community composition and structure of a forest stand (Fekedulegn et al. 2004).

The mixture of categorical and continuous response and predictor variables included in this study suggested the use of classification and regression tree analyses (McCune \& Grace 2002). These analytical techniques are based on decision tree analyses that continually split the experimental units, in a dichotomously branching pattern, into more homogenous groups (De'ath \& Fabricius 2000). The classification or regression tree will be initially "overgrown" but subsequently pruned back based upon cross-validation criteria that suggest optimal tree length for balancing predictive capabilities with model specificity. This technique is particularly useful for exploratory data analysis, as it can identify those independent variables which best split all experimental units into more similar groupings- which are in this study subcompartments.

Trifolium stoloniferum presence, density, and density categories were examined using classification and regression tree analysis for all subcompartments. The effect of disturbance history upon determining T. stoloniferum presence or absence was more explicitly explored with discriminant function analysis. In discriminant function analysis the effect of continuous predictor variables upon a categorical response is explored in an ordination, and the goal is to identify those environmental variables with the strongest relationships to a priori group membership if any exist (McCune \& Grace 2002). 
Because I am only considering presence or absence of $T$. stoloniferum, the output is a 2dimensional ordination with environmental variables displayed as vectors: the direction and strength of relationship with the a priori grouping (presence or absence) is considered.

All statistical analyses were conducted in the open-source statistical software package R 2.8.1 (R Development Core Team 2009). The R package used for classification and regression tree analyses was mvpart (De'ath 2010) and the vegan package (Oksanen et al. 2010) was used for discriminant function analysis.

\section{Results}

Classification tree analysis with $T$. stoloniferum presence or absence within a subcompartment as the categorical response variable identified total harvesting disturbances as the most important predictor (Figure 1; Null error $=0.41$, Model $=0.18$, Cross-Validation strength 0.26 ). Those subcompartments with greater than 4.5 total disturbances were more likely to have $T$. stoloniferum (11 of 16 subcompartments with $T$. stoloniferum), while the great majority of subcompartments with fewer than 4.5 total disturbances did not contain T. stoloniferum (21 of 23 subcompartments without RBC).

A classification tree analysis with $T$. stoloniferum density categories as the response variable similarly identified total disturbances as the main factor that distinguished those subcompartments that had $T$. stoloniferum from those subcompartments that did not (Figure 2; Null error $=0.41$, Model $=0.205$, Cross-validation strength $=0.436$ ). The cross validation for this tree suggested to cut the tree after the first branch (Figure 3), which yielded little information not already offered by the classification tree with T. stoloniferum presence or absence as the response (Figure 1). In 
the interest of data exploration and hypothesis generation, I let the tree grow. The subcompartments that contain T. stoloniferum are further segregated in this tree: those with a western aspect contain 2 of the 3 highest densities, while those with a northeast or northwest aspect contain low or moderate densities. The subcompartments with a total basal area removal since management began of $>172 \mathrm{ft}^{2} /$ acre contained 3 of the 6 moderate $T$. stoloniferum density subcompartments and those with $<172$ square feet/acre removed contained 5 of the 7 low RBC subcompartments.

Regression tree analysis with median crown density as a continuous response variable also identified aspect as important in delineating those subcompartments with higher T. stoloniferum density (Figure 4; Cross-validation error 1.22). The average median density of the 34 subcompartments not facing west was 2.79 . Five subcompartments had a western aspect, and of those, three had a time since last disturbance $($ TSLD) of $>14$ years and two had a TSLD $<14$. Subcompartments with TSLD $<14$ had a mean of 52.3 for median density, while those subcompartments with a TSLD $>14$ had a mean of 5.23 for median density.

The output of discriminant function analysis identified increasing TSLD and logtransformed proportion of basal area removed in the last disturbance as predictors of $T$. stoloniferum absence (Figure 5). Increasing values of log-transformed disturbance total and total basal area removed since management began in 1945 are strongly associated with T. stoloniferum presence. Site index is positively correlated with T. stoloniferum presence, but its influence is not as great as that of other variables. The mean time since last disturbance was substantially higher and total number of disturbances lower in compartments from which T. stoloniferum was absent compared to compartments in 
which it was present (Table 1). The proportion of basal area removed in the last disturbance was lower in compartments in which T. stoloniferum was present $(0.28 \pm 0.1$ of total basal area in T. stoloniferum-present compartments vs. $0.43 \pm 0.3$ in absent compartments) suggesting that more moderate logging disturbances create conditions favorable to T. stoloniferum. Additionally, the total basal area removed since 1950 and the site index were higher as well, suggesting that more fertile and intensively managed compartments were more favorable to T. stoloniferum.

\section{Discussion}

The results of this study suggest that disturbance history is critical in determining T. stoloniferum presence and density within a subcompartment. Total disturbances since management was initiated in these stands was more important in determining the presence or absence of T. stoloniferum, more so than the presence of limestone-derived soils, which have been considered by land managers and plant ecologists as very important for the establishment and persistence of T. stoloniferum (USFWS 2007). Total disturbances is a cumulative variable, and the presence of T. stoloniferum in sites disturbed more than 4.5 times confirms earlier suggestions that moderate, periodic disturbance promote the establishment and persistence of this species (USFWS 2007). In contrast, more intense and less frequent clearcuttings do not promote T. stoloniferum. The majority of subcompartments that have been disturbed less than 4.5 times were either undisturbed control sites or were managed in an even-aged silvicultural system such as clear cutting, while those subcompartments disturbed more than 4.5 times are largely managed in uneven-aged silvicultural systems, such as single-tree selection (Schuler 2004) 
Scheller \& Mladenoff (2002), in a study conducted in northern Wisconsin and the upper peninsula of Michigan, found that plant diversity was higher in forests managed in uneven-aged silvicultural systems with frequent stand entries as compared to old-growth and even-aged forests. The differences in diversity were largely the result of higher light levels found in the uneven-aged forest. The phenomenon of increased diversity and vigor, particularly among herbs flowering after tree leaves have developed, is widely recorded (Pitelka et al. 1980, Moore \& Vankat 1986, Whigham 2004). Reader \& Bricker (1992) conducted a study to follow the response of five forest herbs to forest cuttings of varying size $(0.015,0.053$, and $0.196 \mathrm{ha})$ and intensity $(33 \%$ and $66 \%$ of BA removed). The five herbs all demonstrated unique responses, with different species responding more favorably to different size and intensity combinations. In large openings that had been cut intensively, woody stem competition precluded a vigorous herb response.

The positive response of $T$. stoloniferum to increased disturbance is consistent with the non-equilibrium model of plant coexistence put forth by Pickett (1980). Competitive exclusion by shade-tolerant woody species is characteristic of communities in which succession is allowed to proceed uninterrupted for biologically relevant time periods. But periodic disturbances prevent this exclusion, and those communities that are disturbed regularly will have greater richness and diversity (Collins et al. 1995): this hypothesis is also consistent with the intermediate disturbance hypothesis developed by Connell (1978) in marine systems and extended to terrestrial systems by Huston (1979) and others. The general response of relatively stable communities to episodic or periodic disturbance is an increase in diversity, as resources that would be dominated by more competitive members of the community are liberated. Disturbance in the form of 
forestry-related harvesting activities the Fernow Experimental Forest prevent competitive exclusion and allow for the persistence of $T$. stoloniferum.

\section{Conclusions}

Over the $60+$ year of management history at the Fernow Experimental Forest, those sites that have been disturbed more than 4.5 times are much more likely to support vigorous populations of $T$. stoloniferum; in addition, those sites that have been disturbed

more recently than 14.5 years appear to be more likely to support vigorous occurrences of T. stoloniferum. The results of this study agree with general models of species richness and diversity in forests, such as the non-equilibrium coexistence model put forth by Pickett (1980), in which disturbance maintains diversity by preventing competitive exclusion by shade-tolerant tree species.

\section{Literature cited}

Baker WL (1994) Restoration of landscape structure altered by fire suppression. Conservation Biology 8(3):763-769.

Brawn JD, Robinson SK, \& Thompson FR (2001) The role of disturbance in the ecology and conservation of birds. Annual Review of Ecology and Systematics 32:251276.

Brooks RE (1983) Trifolium stoloniferum, running buffalo clover: Description, distribution, and current status. Rhodora 85(842):343-354.

Chesson P (2000) Mechanisms of maintenance of species diversity. Annual Review of Ecology and Systematics 31:343-66.

Collins SL, Glenn SM \& DJ Gibson (1995) Experimental analysis of intermediate disturbance and initial floristic composition: decoupling cause and effect. Ecology 76:486-492.

Connell JH (1978) Diversity in tropical rain forests and coral reefs. Science 199(4335): 1302-1310. 
De'ath G (2010) mvpart: Multivariate partitioning. R package version 1.3-1. http://CRAN.R-project.org/package=mvpart

De'ath G \& KE Fabricius (2000) Classification and regression trees: A powerful yet simple technique for ecological data analysis. 81(11):3178-3192.

ESRI (Environmental Systems Resource Institute). 2009. ArcMap 9.2. ESRI, Redlands, California.

Fekedulegn D, Colbert JJ, Rentch JR \& KW Gottschalk (2004) Aspect induced differences in vegetation, soil, and microclimatic characteristics of an Appalachian watershed. Castanea 69(2):92-108.

Huston M (1979) A general hypothesis of species diversity. The American Naturalist. 113(1):81-101.

Hobbs RJ \& Huenneke LF (1992) Disturbance, diversity, and invasion: Implications for conservation. Conservation Biology 6(3):324-337.

Lamson N (1987) Site-index class from total height and diameter of dominant and codominant trees in Central Appalachian hardwood stands. USFS Northeastern Forest Experiment Station, Publication NE-RP-605.

Madarish DM, Rodrigue JL \& MB Adams (2000) Vascular Flora and Macroscopic Fauna on the Fernow Experimental Forest. USDA Forest Service Northeastern Research Station General Technical Report NE-291.

Madarish D \& TM Schuler (2002) Effects of forest management practices on the federally endangered running buffalo clover (Trifolium stoloniferum Muhl. ex. A. Eaton). Natural Areas Journal 22(2):120-128.

McCune B \& JB Grace (2002) Analysis of Ecological Communities. MJM Software Design, Gleneden Beach, Oregon, USA.

McNab WH \& PE Avers (1994) Ecological subregions of the United States: section descriptions. Publ. WO-WSA5. Washington, DC: U.S. Department of Agriculture, Forest Service.

Moore MR \& JL Vankat (1986) Responses of the herb layer to the gap dynamics of a mature beech-maple forest. American Midland Naturalist 115(2):336-347.

Oksanen J, Blanchet FG, Kindt R, Legendre P, O'Hara RB, Simpson GL, Solymos P, Stevens MHH \& H Wagner (2010) vegan: Community Ecology Package. R package version 1.17-2. http://CRAN.R-project.org/package=vegan 
Pickett STA (1980) Non-equilibrium coexistence of plants. Bulletin of the Torrey Botanical Club. 107(2):238-248.

Pickett STA, Kolasa J, Armesto JJ \& SL Collins (1989) The ecological concept of disturbance and its expression at various hierachical levels. Oikos 54:129-136

Pitelka LF, Stanton DS \& MO Peckenham (1980) Effects of light and density on resource allocation in a forest herb, Aster acuminatus (Compositae). American Journal of Botany 67:942-948.

Reader RJ \& BD Bricker (1992) Response of five deciduous forest herbs to canopy removal and patch size. American Midland Naturalist 127(1):149-157.

R Development Core Team (2009). R: A language and environment for statistical computing, reference index version 2.8.1. R Foundation for Statistical Computing, Vienna, Austria. ISBN 3-900051-07-0, URL http://www.R-project.org.

Scheller RM \& DJ Mladenoff (2002) Understory species patterns and diversity in oldgrowth and managed northern hardwood forests. Ecological Applications 12(5):1329-1343.

Schuler TM (2004) Fifty years of partial harvesting in a mixed mesophytic forest: composition and productivity. Canadian Journal of Forest Research 34:985-997

Simberloff D (2004) Community ecology: Is it time to move on? The American Naturalist 163(6):787-799.

van der Maarel E (1993) Some remarks on disturbance and its relations to diversity and stability. Journal of Vegetation Science 4:733-736.

USFWS (2007) Running buffalo clover (Trifolium stoloniferum) Recovery Plan: First revision. United States Fish \& Wildlife Service, Fort Snelling, MN.

Whigham DF (2004) Ecology of woodland herbs in temperate deciduous forests. Annual Review of Ecology, Evolution, and Systematics. 35:583-621

Zar JH (1999) Biostatistical Analysis. Prentice Hall, NJ, 663 pp. 


\section{Tables}

Table 1. Mean of predictor variables when $\mathrm{RBC}$ is present or absent.

\begin{tabular}{|l|l|l|}
\hline & RBC Present & RBC Absent \\
\hline & Mean \pm SD & Mean \pm SD \\
\hline \hline Time since last disturbance (years) & $9.75 \pm 4.7$ & $31.13 \pm 29.9$ \\
\hline $\begin{array}{l}\text { Proportion of basal area removed in last } \\
\text { harvest }\end{array}$ & $0.28 \pm 0.1$ & $0.43 \pm 0.3$ \\
\hline $\begin{array}{l}\text { Disturbance total (\# of management } \\
\text { entries) }\end{array}$ & $5.69 \pm 3.2$ & $2.83 \pm 1.6$ \\
\hline $\begin{array}{l}\text { Total basal area removed (since 1950 in } \\
\text { sq.ft/ac) }\end{array}$ & $153.22 \pm 36.1$ & $110.28 \pm 53.7$ \\
\hline Site index & $75.2 \pm 3.8$ & $71.9 \pm 7.4$ \\
\hline
\end{tabular}




\section{Figures}

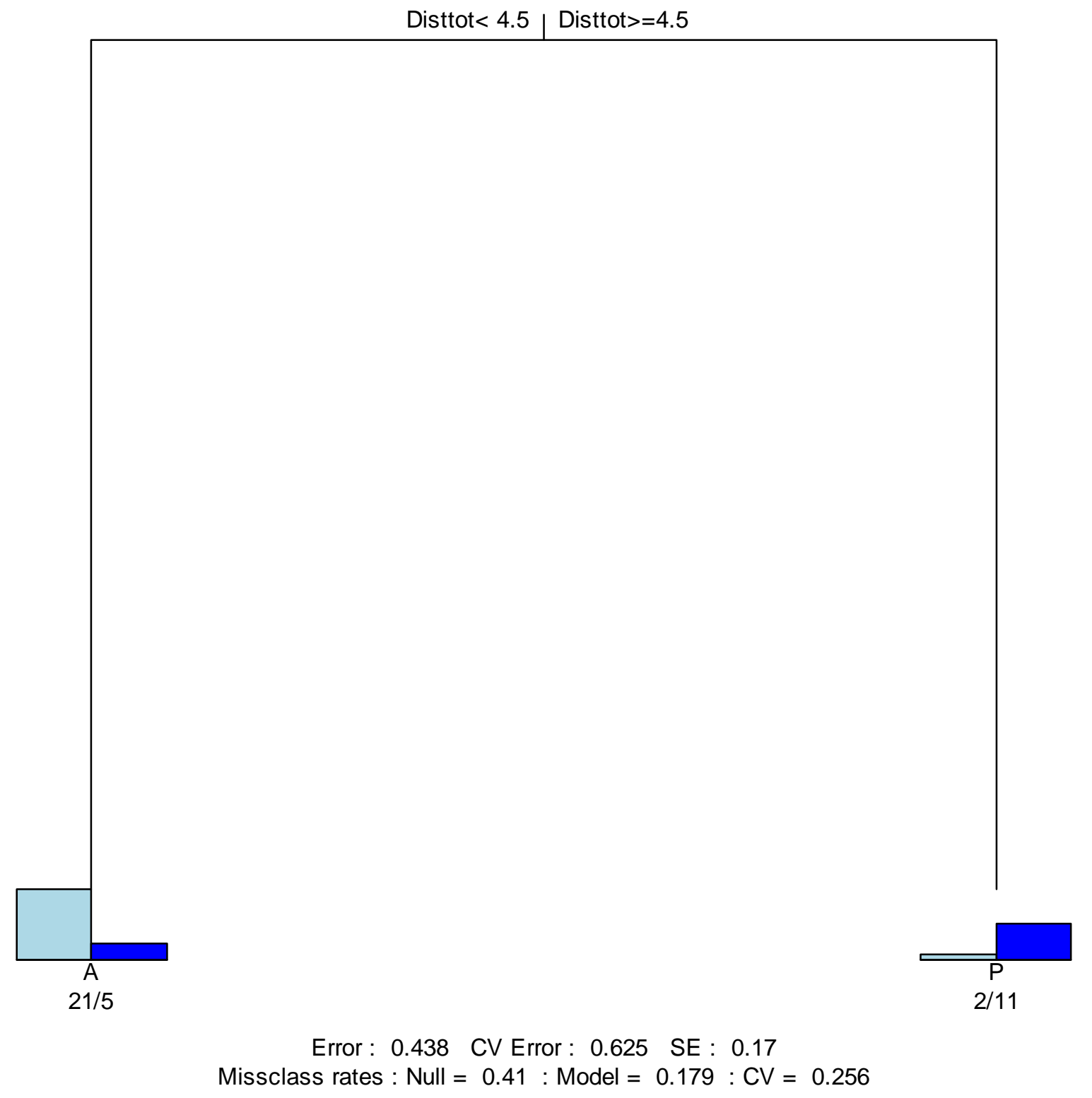

Figure 1. Classification tree analysis of T. stoloniferum presence or absence. The bar graphs present the number of compartments in which $T$. stoloniferum was present or absent, with the absent compartments represented by the left, lighter-colored bar and the present compartments represented by the right, darker bar. Disttot is the total number of disturbances that occurred in a subcompartment since the initiation of management within that unit. 


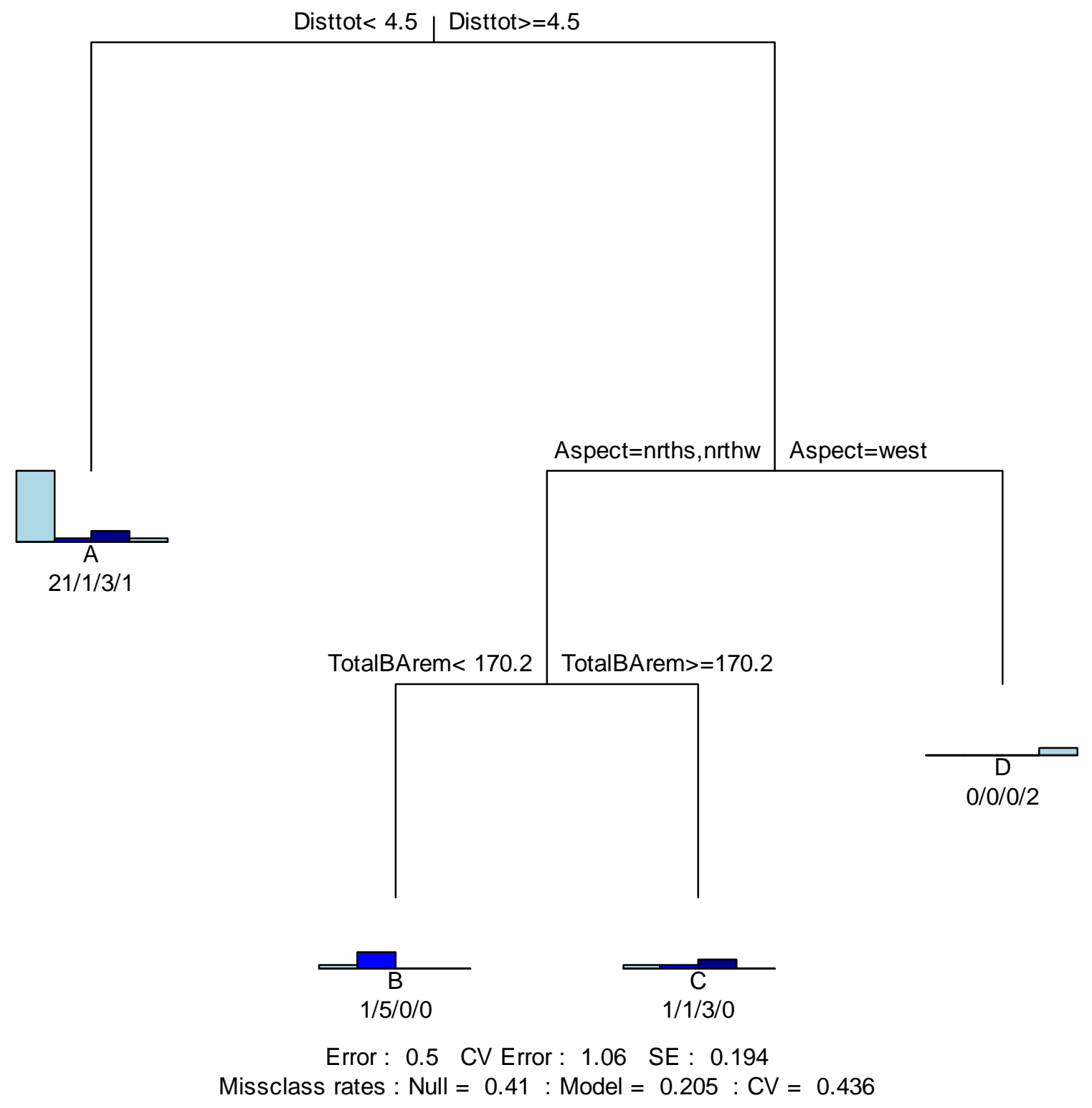

Figure 2. Classification tree analysis of $T$. stoloniferum density category as the response variable. The bars identify the population categories- from left to right they are A (no RBC/acre), B (0.01-10 RBC/acre), C (10.01-35 RBC/acre), and D (>35 RBC/acre). 
Size of tree

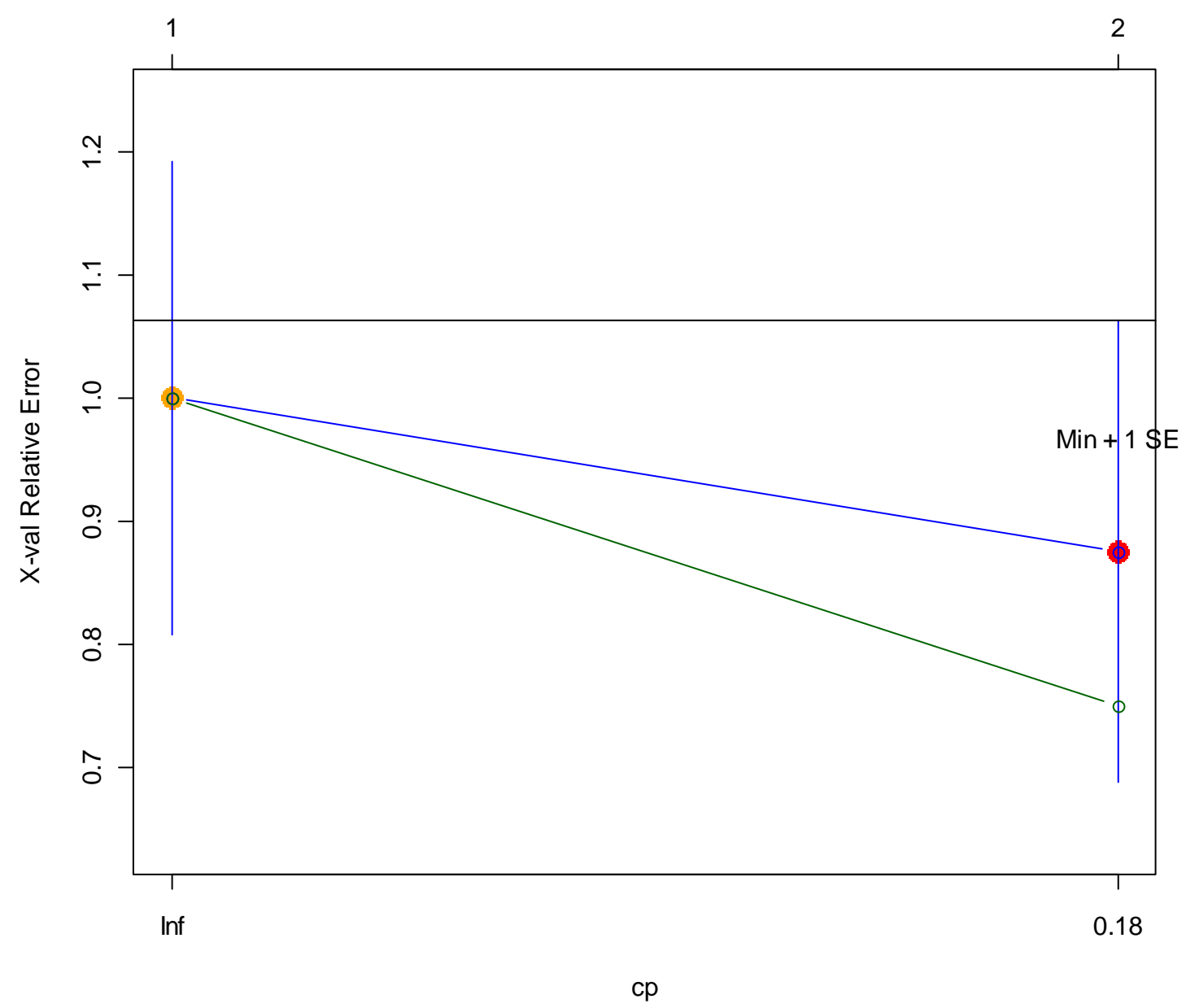

Figure 3. Graphical display of cross validation of classification tree analysis with $T$. stoloniferum density category as the response variable. This figure is interpreted to suggest a tree with one branching and two leaves as optimum tree size. The upper line represents cross-validation relative error. Relative error is represented by the lower line. Tree size can be chosen by picking the smallest tree size, other than 1, in which the relative error is within one standard error of the cross-validation relative error. 
Burkhart 51

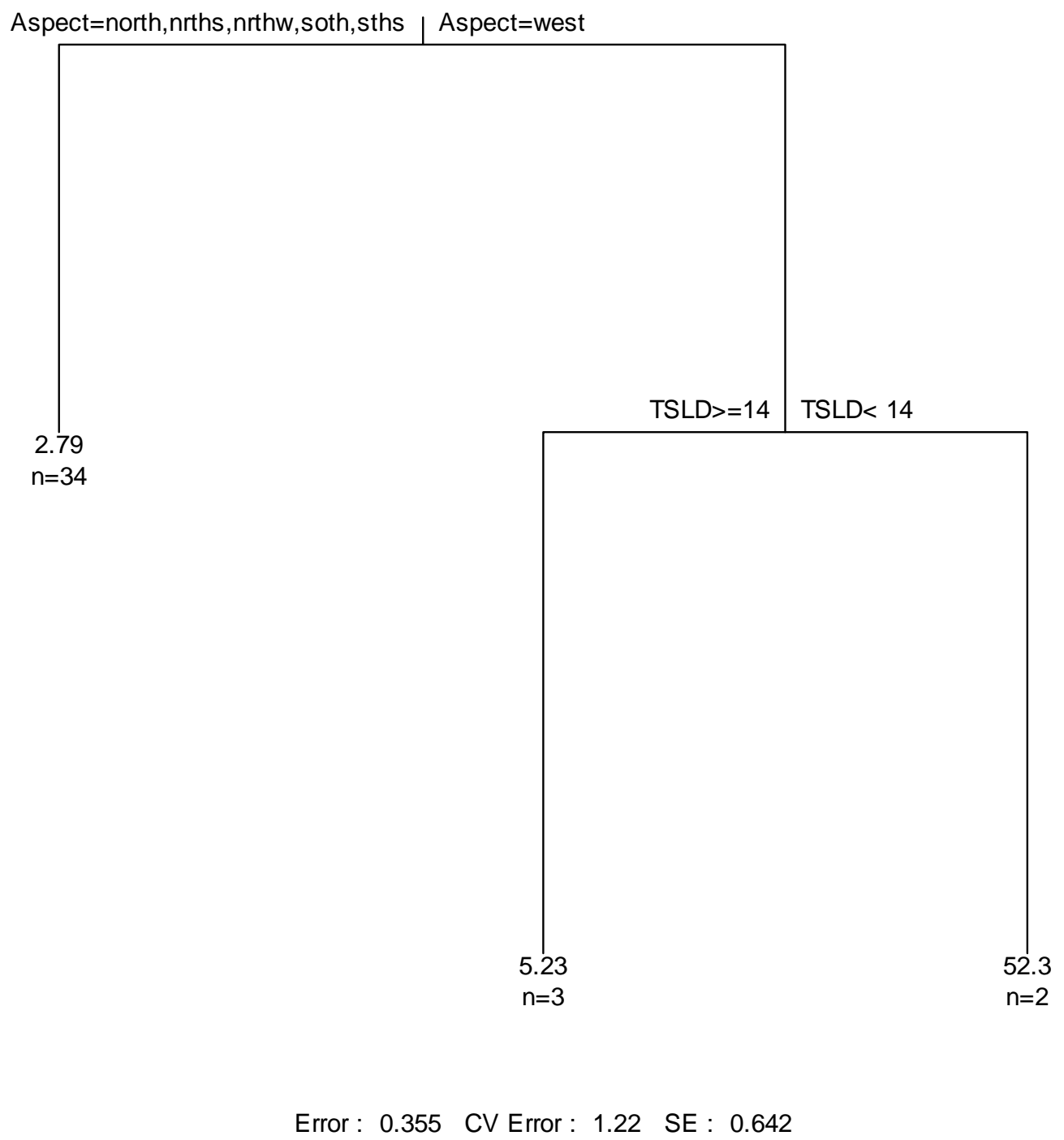

Figure 4. Regression tree analysis with median $T$. stoloniferum crown density as response variable. TSLD refers to time since last logging-related disturbance in years. 
Burkhart 52

Canonical scores



Figure 5. Graphic display of discriminant function analysis with T. stoloniferum presence $(\mathrm{P})$ or absence $(\mathrm{A})$ as the categorical predictor variable and the influence of the predictor variables upon the response. (TSLD $=$ Time elapsed since last disturbance using 2008 as the most recent year; Propremlast $=$ Proportion of basal area removed in the last disturbance; Disttot $=$ The number of management activities that have occurred in a subcompartment; TotalBArem $=$ Cumulative basal area removed in all harvest activities that have occurred in the stand; $\mathrm{SI}=$ Oak site index) 


\section{Appendix}

\section{Appendix- R Code for Statistical Analyses}

\section{DFA}

library(vegan)

RBC=read.table(file="FinalRBCdata", header=T, sep=",")

load("C: $\backslash \backslash$ Documents and Settings $\backslash \backslash$ aira $\backslash \backslash$ My Documents $\backslash \mid$ johnsdocuments $\backslash \backslash$ Quantitative ecology $\backslash$ RBC.RData")

$\mathrm{RBC}$

$\operatorname{attach}(\mathrm{RBC})$

colnames(RBC)

hist(SI)

qqnorm(SI)

shapiro.test(SI)

$\log \mathrm{SI}=\log (\mathrm{SI})$

hist $(\log \mathrm{SI})$

sqrt(SI)

hist(sqrt(SI))

$\log$. habitatRBC=data.frame (cbind(TSLD, $\log 1 \mathrm{p}($ Propremlast $), \log 1 \mathrm{p}($ Disttot $)$,

TotalBArem, SI))

colnames(log.habitatRBC)=c("TSLD", "logPropremlast", "logDisttot", "TotalBArem", "SI")

lda(log.habitatRBC, as.factor(RBCpa), CV=FALSE)

Classification tree analysis with medRBC density categories as the response variable library(mvpart)

RBCcat.tree $=$ mvpart $(\mathrm{RBC}$ cat $\sim \mathrm{SI}+$ minelev + maxelev + Aspect + Greenbrier $+\mathrm{TSLD}+$ Propre mlast+Disttot+TotalBArem, data $=$ RBCcat)

plot(RBCcat.tree)

text(RBCcat.tree)

mvpart (RBCcat $\sim$ SI + minelev + maxelev + Aspect + Greenbrier+TSLD+Propremlast+Disttot +TotalBArem, data $=$ RBCcat, $\mathrm{xv}=$ "none")

mvpart(RBCcat $\sim$ SI+minelev+maxelev+Aspect+Greenbrier+TSLD+Propremlast+Disttot + TotalBArem, data $=$ RBCcat, $\mathrm{xv}=$ "lse")

mvpart $(\mathrm{RBCcat} \sim \mathrm{SI}+$ minelev + maxelev + Aspect + Greenbrier+TSLD+Propremlast+Disttot +TotalBArem, data $=$ RBCcat, $\mathrm{xv}=$ "min")

mvpart (RBCcat $\sim$ SI + minelev + maxelev + Aspect + Greenbrier+TSLD+Propremlast+Disttot

+ TotalBArem, data $=$ RBCcat, size $=5$ )

plotcp(RBCcat.tree)

$\mathrm{xval}=1$ -

plotcp(RBCcat.tree)

$\mathrm{xval}=1$ -

$\mathrm{xval}=1$ -

$\mathrm{xval}=1$ -

plotcp(RBCcat.tree) 
$\mathrm{xval}=10$

$\mathrm{xvmult}=50$

mvpart $(\mathrm{RBC}$ cat $\sim \mathrm{SI}+$ minelev + maxelev + Aspect + Greenbrier+TSLD + Propremlast + Disttot

+ TotalBArem, data $=\mathrm{RBC}$ cat,

$\mathrm{xval}=10, \mathrm{xvmult}=50, \mathrm{prn}=\mathrm{TRUE}$, legend $=$ TRUE, bord=TRUE)

plotcp(RBC.cat)

plotcp(RBCcat.tree)

summary(class.tree)

summary(RBCcat.tree)

$\operatorname{par}(\mathrm{mfrow}=\mathrm{c}(1,1), \mathrm{xpd}=\mathrm{NA})$

text(RBCcat.tree)

text(RBCcat.tree, use.n=TRUE)

Regression tree analysis with medRBC/acre as the response variable.

RBCmed.regress.tree $=$ mvpart $($ medRBC.ac $\sim \mathrm{SI}+$ minelev + maxelev + Aspect + Greenbrier $+\mathrm{T}$ $\mathrm{SLD}+$ Propremlast + Disttot + TotalBArem, data $=\mathrm{RBC}$ cat $)$

RBCmed.regress.tree $=$ mvpart $($ medRBC.ac $\sim \mathrm{SI}+$ minelev + maxelev + Aspect + Greenbrier $+\mathrm{T}$

$\mathrm{SLD}+$ Propremlast + Disttot + TotalBArem, data $=\mathrm{RBC}$ cat, size $=3)$ )

plotcp(RBCmed.regress.tree)

plot(RBC.med.regress.tree)

text(RBCmed.regress.tree)

Classification tree analysis with RBC presence or absence as presence variable

RBCpa.tree $=$ mvpart $($ RBCpa $\sim$ SI + minelev + maxelev + Aspect + Greenbrier + TSLD + Proprem last+Disttot+TotalBArem, data $=$ RBCcat $)$

$\mathrm{RBCpa}$. tree $=$ mvpart $(\mathrm{RBCpa} \sim \mathrm{SI}+$ minelev + maxelev + Aspect + Greenbrier $+\mathrm{TSLD}+$ Proprem last + Disttot + TotalBArem, data $=$ RBCcat, $x v a l=10, x v m u l t=50, \mathrm{prn}=\mathrm{TRUE}$, all.leaves $=$ TRUE, legend $=$ TRUE, bord $=$ TRUE)

plotcp $(\mathrm{RBCpa}$.tree) 


\title{
Chapter III
}

\section{Habitat assessment of Trifolium stoloniferum (Muhl. ex A. Eaton): Relationship of patch abundance and flowering success to environment}

\begin{abstract}
Trifolium stoloniferum Muhl. ex A. Eaton is a federally endangered plant species of the family Fabaceae. To facilitate conservation and management of this species I qualitatively and quantitatively describe its habitat. I conducted detailed habitat assessment of T. stoloniferum "patches"- or discrete occurrences- at the Fernow Experimental Forest in West Virginia, U.S.A. Patches were selected in a stratified random manner from all T. stoloniferum patches within the Experimental Forest, and selected patches were representative of the range of abundance and flowering success. Control sites were also assessed, which were either sites from which T. stoloniferum had been extirpated or sites that met basic environmental criteria of $T$. stoloniferum but in which it had never been detected. A combination of hemispherical photography and analysis, vegetation sampling and measurement, physiographic measurements, and site disturbance history were employed to describe T. stoloniferum patches. Patches were categorized into groups based on abundance of rooted crowns and relative flowering success. Multi-response permutation procedures revealed significant differences among crown and flowering groups based upon herbaceous community composition, and MANOVA revealed that the measured environment differed significantly among groups. Nonmetric multidimensional scaling was employed to synthesize and visually display the relationships between community composition and environmental variables.
\end{abstract}


Classification tree analyses demonstrated that plant community diversity and structure, photosynthetically active light levels, and time since disturbance all interact to affect habitat quality for T. stoloniferum- emphasizing the importance of disturbance in providing an environment suitable to $T$. stoloniferum and describing the community structure and habitat characteristics resulting from appropriate disturbance. The results of this study provide indications of habitat quality for $T$. stoloniferum and provide clear guidelines for habitat management.

Keywords: Running buffalo clover, Fernow Experimental Forest, multi-response permutation procedure (MRPP), nonmetric multidimensional scaling (NMDS), classification tree analysis, canopy disturbance, hemispherical photography, plant community.

\section{Introduction}

Trifolium stoloniferum Muhl. ex A. Eaton is a federally endangered plant species of the plant family Fabaceae (see Brooks 1983, Figure 1). It has been endangered due to a variety of causes, including loss of animal dispersers and habitat change and loss (Campbell et al. 1988, USFWS 2007). A high priority of the integrated conservation efforts of $T$. stoloniferum is to increase understanding of the ecological setting in which this species occurs (USFWS 2007). Biological habitats are complex in structure, composition, and function, and the identification of the few variables most important in determining success of a species is essential to effective conservation (MacNally 2002). Management efforts are most likely to succeed (or be undertaken at all) when clear and achievable objectives that will elicit a positive species response can be identified (Tear et al. 2005). 
Previous work concerning T. stoloniferum ecology has focused on diverse areas, including genetics (Crawford et al. 1998), dispersal biology (Ford et al. 2003), pollination biology (Franklin 1998, Taylor et al. 2004), nitrogen-fixation abilities (Morris et al. 2002), historical ecology (Campbell et al. 1988), and soil chemistry (Hattenbach 1996). The work of Madarish \& Schuler (2002) informed the current study: they examined the response of $T$. stoloniferum to disturbance in the form of logging and associated skidding of logs from the forest. They found that patches of $T$. stoloniferum experienced declines immediately after the disturbance but that abundances returned to pre-disturbance levels over the course of five to seven years. This study will build upon their work by quantitatively considering how reproduction and patch abundance are related to the environment, particularly those environmental variables influenced by disturbance to the substrate and forest structure. In addition, this study will emphasize the management of T. stoloniferum in the context of principles of forest and patch dynamics.

\section{Methods}

To identify those factors contributing most to T. stoloniferum population growth or decline, I conducted a detailed habitat assessment of $T$. stoloniferum patches of varying levels of abundance and reproductive success. I measured vegetation composition and structure, canopy structure and light levels, physiographic characters, substrate composition, and disturbance history. These variables can be easily measured by experienced plant ecologists and land managers, except for canopy structure which can be estimated and disturbance history which will express itself in the extant vegetation (White 1979). 
Study site description and location

The Fernow Experimental Forest in West Virginia (latitude 39³'15" N, longitude $79^{\circ} 41^{\prime} 15^{\prime \prime} \mathrm{W}$ ) is a research forest established in 1934 approximately $2.5 \mathrm{~km}$ south of the town of Parsons, WV (Madarish et al. 2002). The Fernow contains 1902 ha of largely forested habitat ranging from 533 to $1,112 \mathrm{~m}$ above sea level. It is a part of the Monongahela National Forest, an approximately $3,719 \mathrm{~km}^{2}$ forest stretching southwest to northeast across the mountainous regions of West Virginia.

The Fernow Experimental Forest has been conducting silvicultural, ecological, and watershed research since 1933 (Madarish et al. 2002). A large component of the Forest's mission has been to implement and maintain silvicultural practices at the level of the management unit within the forest. The fundamental unit of management at the Fernow is either the watershed or compartment- with compartments having been delineated based on site characteristics. Compartments are in some cases further divided into subcompartments, and silvicultural treatments are applied at either this level or at the compartment level.

Trifolium stoloniferum is currently found in approximately 67 extant "patches" in 15 subcompartments or watersheds. All but one of the patches occurs in a subcompartment or watershed underlain by calcareous soils. The disturbance that appears to have been most influential in maintaining T. stoloniferum is periodic logging and associated skidding. Trifolium stoloniferum is most likely dispersed by the logging equipment as well, as T. stoloniferum is found largely along skid roads that are used episodically (at intervals from 10-50 years) to remove timber from the forest. Trifolium stoloniferum patches also occur on maintained Forest Service roads and some patches are 
situated along paths maintained by foot travel. All T. stoloniferum patches at the Fernow occur where the soil has been disturbed by some means, either equipment and vehicle traffic, foot traffic, and some minor patches are associated with deer trails. Trifolium stoloniferum was discovered on the Fernow in 1993, and monitoring of T. stoloniferum by personnel of the Fernow has been conducted since 1998.

Inflorescence count and site selection

Trifolium stoloniferum was first detected at the Fernow Experimental Forest in 1993, and, beginning in 1998, occurrences were censused annually until 2004. Since 2004 , censuses have been conducted once every two years for every occurrence. Some of the patches censused in 2008 were heavily disturbed during the winter of 2008-2009 by logging activity; consequently, these patches were not included in detailed studies conducted in 2009 nor were any inflorescences and very few rooted crowns found in these patches in 2009. I counted inflorescences in all extant T. stoloniferum patches in May and June 2009.

Patches and control sites were selected for detailed habitat assessment based upon censuses of patches conducted in 2008 or 2009 and inflorescence tallies. The patches were chosen in a stratified random approach to represent a gradient of patch size and inflorescence production. Nine combinations of patch size (range: 2 to 565 rooted crowns) and relative inflorescence production (range: 0 inflorescences produced by 227 rooted crowns to 27 inflorescences produced by 16 rooted crowns) were identified and replication was achieved for all but one treatment combination. The combinations and the number of replicates (in parentheses) were as follows: low population/low flowering (3), low population/medium flowering (l-no replication), low population/high flowering 
(4), medium population/low flowering (4), medium population/medium flowering (5), medium population/high flowering (4), high population/low flowering (3), high population/medium flowering (3), and high population/high flowering (4). Population size is determined by counts of rooted crowns, which is the technique for population size determination recommended by the USFWS Recovery Plan (USFWS 2007). Low, medium, and high rooted crown abundance by patch were 2-30, 31-99, and $>100$ rooted crowns, respectively. Flowering success categorization was based upon flowering index, an index I developed to assess relative reproductive success. Flowering index was equal to number of inflorescences/number of rooted crowns (Flowering index $=$ inflorescences/rooted crowns). Low, medium, and high flowering index were $0-0.05$, $0.06-0.19$, and $>0.20$, respectively.

Additionally, eleven sites were chosen as control sites. There were two categories of control sites. One category of sites was chosen because they had at one point supported T. stoloniferum but the species had become extirpated- this category was replicated four times. The second category of controls consisted of sites that had never been known to support T. stoloniferum but appeared to meet basic environmental requirements of the species. In particular, these sites were situated along skid roads underlain by Greenbrier limestone, characteristics shared by 30 out of 31 sites with extant populations of $T$. stoloniferum. The selection process consisted of creating a GIS in ArcMap 9.2 (ESRI 2009) that included Fernow skid roads, bedrock geology, and management unit boundaries, then moving a cursor over a point along a skid road in a management unit that had never contained T. stoloniferum. The geographic coordinates of the point were 
recorded and I then navigated to this point in the field and established a habitat assessment plot.

A total of 31 extant subpopulations and 11 control sites were included in the study. See Appendix 1 for a complete list of sites used in this study, the number of rooted crowns and inflorescences, flowering index, and treatment category. Additionally, see Appendix 2 for photographs and descriptions of sites selected to be representative of different levels of T. stoloniferum success.

Plots were established around T. stoloniferum patches and in control sites. Plot design was a modification of the North Carolina Vegetation Survey (Peet et al. 1998, Figure 2). The center of each plot was located approximately in the middle of the RBC patch- there was some flexibility in this requirement to accommodate populations of varying size. Plots consisted of a single square $100 \mathrm{~m}^{2}$ quadrat. All T. stoloniferum patches included in this study were associated with a path of some type- either an improved road, skid road, foot trail, or animal path. Because the majority of subpopulations in the Fernow occur on skid roads, 36 of the 42 sites selected for inclusion in this study were also situated on skid roads. Plot design was influenced by this factor, as a square shaped plot was well-suited to proportionately represent the road, its margin, and the forest floor which the road bissected. The degree to which the road affected the surrounding vegetation and substrate varied among plots, but road effects, such as erosion and disturbance to vegetation, were usually most substantial on the downhill side of steep roads. Additionally, roads had biotic, and thus structural, effects by dispersing native and non-native plants. Roads often appeared to serve as corridors for deer and bear, who were detected by myself and others moving along the roads. 
Additionally, they served as corridors for humans who walk the roads in search of plants and animals in addition to Fernow Experimental Forest personnel during the conduct of their duties.

\section{Habitat assessment}

Detailed habitat assessment of $T$. stoloniferum patches occurred from June-August 2009. Habitat assessment consisted of five main activities- canopy photography, measurement of physiographic features, vegetation assessment, substrate assessment, and a determination of the time since last disturbance for each subpopulation. Each of these activities is discussed in detail below.

Canopy photography was conducted on clear to overcast days in mid-June to early July for the majority of sites. Several control sites were photographed in August 2009. The photographs were taken with a Nikon E8400 digital camera with a fisheye lens attached. The camera was mounted to a self-leveling mount, which was attached to a sturdy tripod. Analysis of hemispherical photographs required that photographs be oriented with the top center of the photograph towards magnetic north, which along with determination of aspect and geographic position allows for accurate determination of incident solar radiation across time and space.

Analysis of canopy photographs was accomplished using the software WinsCanopy 2006a (Regent Instruments Inc. 2006). Before canopy analysis can occur, all photographs had to be preprocessed in order to exclude non-canopy elements from analysis. For example, "masks" must be created for ground that is included in the hemispherical photograph because of slopes as well as tree trunks and large branches or other substantial woody vegetation. In addition, the geographic position, in the form of 
latitude and longitude, as well as the date and time of photographing are entered. This information allows for the software to simulate the position of the sun over the course of a calendar year or other pre-selected time interval. After photographs are processed, they are analyzed for a variety of structural and light environment characteristics. The variables I included in the statistical analysis process included gap fraction, canopy openness, leaf-area index, and total photosynthetic photon flux density, as these are most easily understood, visualized, and recreated by forest managers seeking to stimulate growth and reproduction of $T$. stoloniferum.

Slope and aspect were measured from the center of the habitat assessment plot. Slope was assessed with a clinometer. Aspect was determined with a compass by orienting the compass towards the steepest downhill slope and recording the direction in degrees. Although many of the habitat assessment plots were centered in a skid road, I attempted to measure the aspect and slope of the larger hill upon which the plot was situated and not the slope of the road.

Vegetation assessment consisted of several components. Basal area of trees greater than 4 inches in diameter (10.16 centimeters) was assessed using a 10 factor basal area prism positioned at the center of the habitat assessment plot. All "borderline" trees were checked by measuring distance from plot center to the center of the tree. If DBH * 2.75 was greater than distance to the tree, the tree was counted as "in". The number of trees tallied from plot center was multiplied by 10 to provide an estimate of basal area in $\mathrm{ft}^{2} /$ acre around the RBC subpopulation.

Saplings within the plot were tallied by species. A tree was considered a sapling if it had a DBH of less than $10 \mathrm{~cm}$ and was greater than 1 meter in height. Shrub density 
was assessed by means of two diagonal transects stretching between the four corners of the plot. The transect was created by stretching a metric tape between two diagonal corners. The distance in meters which a shrub overlapped the transect was recorded along with the name of the species.

Herbaceous vegetation was assessed by means of five $1 \mathrm{~m}^{2}$ circular subplots positioned purposefully within the plot. Three of the sub-plots were positioned down the center of the plot, at 2, 4, and 8 meters from the downhill center of the plot (see Figure 2). This often meant that these sub-plots were positioned within the center of the skid road in which the T. stoloniferum sub-population occurred. The two remaining subplots were positioned perpendicular to the other three: one was positioned along the downhill "road margin," which appeared to be an area where T. stoloniferum crowns were concentrated; and one uphill positioned $4 \mathrm{~m}$ from plot center, which usually caused this subplot to be positioned in the forest floor, or, if the road was wide, in the brushy margin of the road. Herbaceous vegetation percent cover was estimated for each species occurring in the $1 \mathrm{~m}^{2}$ subplot. Percent cover is estimated by mentally projecting the total surface area of all the parts of a species of plant on the ground (Peet et al. 1998).

Leaf litter depth was measured and substrate composition estimated for all subplots. Leaf litter depth was measured by poking a small hole in the litter to identify where the litter layer ended and the organic or mineral soil layer began. Then, I inserted a metal ruler flush with the bottom of the litter layer and recorded the height of the litter layer. I repeated this three times for every $1 \mathrm{~m}^{2}$ subplot-at 120, 240, and 360 degrees compass bearing-to obtain an average for the subplot. Substrate composition was 
estimated by percentage for each subplot. The substrates encountered were of six types: leaf litter, mineral soil, coarse woody debris, moss, rock, and crushed gravel.

The principal disturbance to T. stoloniferum patches in the Fernow Experimental Forest is in the form of forestry harvesting operations. These activities affect $T$. stoloniferum by opening up the canopy, disturbing litter and soil, moderately compacting soil, dispersing seeds of many plants on the logging equipment, and indirectly by influencing the response of vegetation. The years since last disturbance were estimated for each plot by examining forestry records for the Fernow Experimental Forest. In plots that may have been disturbed by skidder traffic without simultaneous disturbance to the canopy (skidders traveled over the subpopulation en route to a harvesting activity but no harvesting occurred at that site), I consulted an experienced forestry technician at the Fernow to assist in determining the most recent year in which the subpopulation was disturbed.

\section{Data processing and analysis}

There were 10 treatment groups which I used to ensure accurate representation from all possible $T$. stoloniferum patch types- the factorial combination of low, medium, and high abundance of rooted crowns and low, medium, and high flowering index, which gave 9 treatment groups plus a control group. There were 42 total patches assessed, including controls, so 10 treatment groups gave limited replication. This would give low power to efforts to find differences between groups. Consequently, I assigned every patch to a Crowngroup and a Flowergroup, based upon patch abundance and flowering index, respectively. Groups based upon crowns were Crowngroup 0 ( 0 crowns- 11 replicates), 1 (1-30 crowns - 9 replicates), 2 (31-99 - 12 replicates), and 3 ( $\geq 100$ - 10 
replicates). Groups based upon flowering success were Flowergroup 0 (Flowering index $=0$ - 17 replicates), 1 (0.01-0.05 - 4 replicates), 2 (0.06-0.19 - 8 replicates), and 3 $(\geq 0.20$ - 13 replicates).

The first step in data analysis was summarization and examination/evaluation of environmental variable means and standard deviations for Crowngroups and Flowergroups. This allowed for the initial assessment of which variables may be important in determining RBC patch size and flowering success. In other words, the means were screened for explanatory power and their inclusion in subsequent multivariate analyses was influenced by these differences in means.

In addition, those variables which did not contribute substantially to patch size or flowering success were eliminated when looking for global differences among response groups (such as in multivariate analysis of variance (MANOVA)). Additionally, variables which appeared to have little explanatory capability were excluded from multiple regression approaches (such as classification and regression tree analysis), thus allowing for greater parsimony in multivariate models, and less of a "data fishing" approach (Daudin 1986).

A potential downfall of data screening is the introduction of bias or the inability to detect interactions and collinearity among variables (MacNally 2002). However, I would argue that bias is introduced no more than in the process of selecting which variables to measure. Inspection of means serves the simple role of summarization of observations that are difficult to imagine without numeric support. Also, collinearity can be substantially reduced by model parsimony. The initial process of screening variables for their predictive capability will allow for the selection of one variable among a set that is 
likely to be collinear. For example, in the canopy analysis process I generated measures for canopy openness, gap fraction, leaf area index, and light density at instrument height (measured in units of photosynthetic photon flux density, or PPFD). These variables, particularly canopy openness, gap fraction, and leaf area index are all direct measures of canopy structure and thus likely to be highly collinear. PPFD is obviously affected by canopy structure, but is also influenced by topographic position, and collinearity would in theory be less than between the direct measures of canopy structure. The inclusion of only one of these variables in a predictive model will reduce collinearity. Variable selection should be based on apparent strength of relationship, literature precedence and thus the ability to compare to other studies, and ease of interpretation.

Based upon initial screenings of data, I eliminated variables for multivariate models that demonstrated no clear relationship to Crowngroup or Flowergroup or were likely to demonstrate collinearity with other variables. These variables were slope, measures of percent cover for substrates, measures of leaf litter depth, gap fraction, and leaf area index.

To test for global differences among Crowngroups and Flowergroups based upon environmental variables I performed a MANOVA analysis in R 2.10.0 using the manova function found in the base package (R Core Development Team 2009). MANOVA is able to address the question of whether all variables collectively vary among levels of a factor by comparing within group variation to among group variation, similar to ANOVA. But unlike a sequence of univariate ANOVA tests, MANOVA is able to address covariance among response variables. In constructing the model collinearity should be avoided as much as possible and redundant variables should not be used. 
Variables were checked for univariate normality by means of histograms, normal Q-Q plots, and the Shapiro test and transformed to approximate normality if appropriate. See

R Code Appendix for analysis code. Here is the structure of the MANOVA model:

Response variable (Crowngroup or Flowergroup) log transformed time since last disturbance (TSLD) + basal area (BA) + log plus 1 transformed sapling tally (SAPTALLY) + log plus one transformed shrub cover (SHRUBCOV) + total herbaceous cover (TOTALVEGCOVER) + canopy openness (OPEN) + light density (PPFD) + Shannon's diversity index (SHANNON).

Understory plant community assemblage data were summarized to express the mean percent cover of a plant species across 5 subplots within the main plot. After eliminating "rare" species that occurred in $\leq 4 \mathrm{RBC}$ main plots (see McCune \& Grace 2002), nonmetric multidimensional scaling (NMDS) was used to ordinate plant communities sampled from each RBC patch. After eliminating uncommon plants, 67 vascular plant species remained. The Bray-Curtis distance measure was used to construct the similarity matrix of sites for the ordination. Dimensionality of the ordination was chosen to be $\mathrm{k}=3$, based upon measures of stress and a desire for ordination outputs to be readily interpretable (stress $=18.38,2$ convergent solutions found after 16 tries). Scaling consisted of centering, PC rotation, and halfchange scaling. All NMDS procedures were performed with the statistical package R version 2.10.0 (R Core Development Team 2009) using the vegan package.

An environmental fitting function (envfit) was performed in which environmental variables are projected as vectors into ordination space and then assessed for their relationship to the ordination surface (Oksanen 2010). The strength of this relationship is measured and assigned a value analogous to an $r^{2}$ goodness of fit, and this value is assigned a $p$ value based upon 1000 random permutations wherein environmental 
variables are randomly assigned to different sites (see R Code Appendix). The envfit function is univariate- it only considers the relationship between one variable and ordination output at a time. This table can be interpreted very similarly to a regression output. NMDS1 and NMDS2 refer to the regression coefficients for each variable, $\mathrm{r}^{2}$ is directly analogous to the $r^{2}$ in a typical regression, and a $\mathrm{p}$ value is given based upon permutations in which a value of the variable is randomly attributed to a site. The variables assessed for fit were the same "parsimonious" variables used in the MANOVA analysis, but this time left untransformed (and aspect was also included): aspect, time since last disturbance, basal area, sapling tally, shrub cover, total herbaceous cover, canopy openness, photosynthetic photon flux density, and Shannon's diversity index.

Ordination outputs were displayed in 2 dimensions, although the actual dimensionality of the ordination was $\mathrm{k}=3$. This does not seem to interfere with the emergence of clear, logical patterns in the output. Ordination outputs were labeled using either Crowngroup or Flowergroup categories, so that the position of each site and its associated vegetative community is distinguished by its membership in a Crowngroup or Flowergroup. Ordination outputs were then overlain by environmental variables, the results of which are presented in Figures 3-4 as vector overlays and Figures 5-13 as surface overlays. I chose to include measures of $T$. stoloniferum success Crowngroup and Flowergroup as environmental variables as well, since these would not by default be highly correlated with ordination structure, which was organized based upon herbaceous community composition, of which $T$. stoloniferum was often but a small part.

To discern if there were statistically significant differences between patch groups and flowering index groups based upon community composition, I applied a multi- 
response permutation procedure of within vs. among group similarities (MRPP). The data input to the analysis was a data matrix of percent cover of herbaceous species at each site - the five subplots assessed at each site were pooled and the mean percent cover by species was determined. MRPP used the same matrix of 67 vascular plant species that occurred in 5 or more of the $42 \mathrm{RBC}$ plots that was used in the NMDS procedure. The distance matrix of Sorenson distances was rank-transformed. Test statistic $\mathrm{T}$ is the difference between observed and expected mean distance and the standard deviation of the expected difference. The p-value is the probability that the T-statistic is the result of chance alone. The A statistic is a measure of effect size- it is a description of chancecorrected within-group agreement. MRPP was conducted in PC-ORD 5.10 (McCune \& Mefford 2006).

To further discriminate among environmental variables and assess whether highlevel interactions among variables structure response, I performed classification tree analyses with Crowngroup and Flowergroup as the factor response variables. The regression tree analyses were performed in $\mathrm{R}$ version 2.10 .0 with the mvpart package, and the $\mathrm{R}$ code used is outlined in the R Code Appendix. The model structure is as follows:

Response (Crowngroup or Flowergroup) Time since last disturbance (TSLD)+ basal area (BA)+ sapling tally (SAPTALLY)+ shrub cover (SHRUBCOV)+ total herbaceous cover (TOTALVEGCOVER)+ canopy openness (OPEN)+ light density (PPFD) + Shannon's diversity index (SHANNON).

Classification tree analyses are used to identify those environmental variables most strongly associated with a chosen response variable. De'ath and Fabricius (2000) outline the application of this technique in ecology and provide concise explanations of how it works. Fundamentally, a single environmental variable is selected from all 
available environmental variables to partition the collection of sites into two branches that are more homogenous than before the split. This process is repeated until the tree is grown to an adequate length. Tree length is determined by a variety of techniques, and the effectiveness of the process in creating homogenous "leaves" of the tree is assessed through measures of cross-validation error.

Finally, I employed indicator species analysis to test whether T. stoloniferum was associated with specific members of the regional flora more commonly than others. Indicator species analysis follows that of Dufrene \& Legendre (1997), where a species affiliation with a response group (determined either a priori or through cluster analysis) is quantified. Affiliation is measured in terms of indicator index value- a perfect indicator would only occur in a specific group and would occur in every plot of that group. Statistical significance of indicator value is determined through randomized permutations, which allows for the calculation of a $\mathrm{p}$ value. $\mathrm{P}$ values are calculated through $\mathrm{p}=(1+$ number of runs $>=$ observed $) /(1+$ number of randomized runs $)$, and interpreted for the group in which a given species has its greatest importance value.

\section{Results}

\section{Data screening}

Means of environmental variables by Crowngroup and Flowergroup are displayed in Tables 1 and 2, respectively. A detailed inspection of these variables for Crowngroups (Table 1) reveals several variables that had a strong relationship to abundance of rooted crowns within a patch. 1) Time since last disturbance decreased as patch abundance increased. 2) Basal area showed a decreasing trend, except for Crowngroup 3 which was heavily influenced by a patch along an improved road that had a basal area of 210 
$\mathrm{ft}^{2} /$ acre. 3) Shrub cover increased as patch abundance increased; total herbaceous cover was positively correlated with Trifolium stoloniferum abundance. 4) Photosynthetic photon flux density showed an increasing trend as patch abundance increased. 5) Shannon's index of diversity showed an increasing trend as patch abundance increased. 6) Gap fraction and canopy openness revealed either no trend or a negative association between these two measures of canopy "perforation" and patch RBC abundance.

An assessment of means of environmental variables by Flowergroups reveals trends similar to yet distinct from Crowngroups (Table 2). 1) Time since last disturbance decreased as flowering success increased. 2) Basal area showed a decreasing trend as flowering success increased, with a larger difference between those plots that contained no flowers and those experiencing low flowering success. 3) Sapling tally demonstrated a moderate negative trend as flowering success increased. 4) Shrub cover increased markedly as flowering success increased. 5) Total herbaceous cover increased dramatically as some flowers were found in the plot, but there was little difference between low, medium, and high flowering success patches.

It must be noted that means for Crowngroup and Flowergroup 0 could be difficult to interpret for canopy variables, as these groups included two control sites which were relatively recent clearcuttings and thus had very sparse canopies- as a consequence, these groups have inflated values and large standard deviations for gap fraction, openness, and photosynthetic photon flux density. So, with this in mind, I can interpret these variables as having a clear positive relationship with flowering success. Shannon's index of diversity also increased with flowering index. 
Control sites (Crowngroup 0) could be distinguished by whether they had ever contained T. stoloniferum (these sites were selected along skid roads in rich, mesic forests over calcareous soils) or if they had contained T. stoloniferum but no longer did. I wanted to know if environmental or disturbances variables distinguished these groups of sites from each other. Table 3 suggests that there are no important differences between these two types of control groups.

\section{MANOVA}

MANOVA revealed significant differences among the environmental variables associated with different levels of Crowngroup and Flowergroup (Tables 4a and b). These global differences support the assumption that environmental differences do exist among sites with varying population sizes and relative reproductive success, and that the variables measured and included in the model are able to adequately represent these differences.

Nonmetric multidimensional scaling

Nonmetric multidimensional scaling outputs demonstrate the strong, yet complex, relationships between $T$. stoloniferum success, environmental gradients, and the herbaceous community (Figures 3-4). Basal area and time since last disturbance are negatively associated with successful $T$. stoloniferum sites, while canopy openness, sapling tally, light levels (PPFD) shrub cover, total vegetative cover, shrub cover and herbaceous diversity are positively associated with $T$. stoloniferum abundance and reproductive success. Figure 3 and Figure 4 are ordination outputs presented with strongly correlated environmental variables $\left(\mathrm{r}^{2}>0.5\right)$ as vectors, with the direction of the vector indicating its direction of association within the ordination "landscape" and the length of the vector indicating the magnitude of its association. The results of the 
environmental fitting function that determined which variables were significantly correlated with ordination structure are presented in Table 5. Ordinations are presented with Crowngroups (Figure 3 \& Figures 5-8) and Flowergroups (Figure 4 \& Figures 9-13) as labels for individual sites. Figures 5-13 highlight individual environmental variables by displaying them as a gradient across the ordination landscape.

Either a long time since last disturbance (TSLD) or an extremely short time since last disturbance is associated with sites categorized as Crowngroups 0 and 1 (Figure 5). Sites labeled as Crowngroup 2 or 3 (higher abundance patches) appear to be associated with disturbances that have occurred between 3 and 11 years.

Sites with high abundance of $T$. stoloniferum (Crowngroups 2 and 3) generally have higher levels of herbaceous diversity (Figure 6). The relationship between canopy openness and Crowngroup was less apparent, although it does appear that the majority of high abundance sites have a canopy openness between 8 and 10\% (Figure 7).

Basal area (BA) shapes vegetative response (Figure 8) and Crowngroup is related to vegetative response. Sites with higher abundance of $T$. stoloniferum (Crowngroups 2 and 3) are associated with basal areas between 80 and $110 \mathrm{ft}^{2} / \mathrm{ac}$, with some exceptions.

Sites with medium-to-high flowering success (Flowergroups 2 and 3) are clearly associated with sites that possess a greater diversity of herbaceous vegetation (Figure 9). Sites with medium-to-high flowering success also appear to have been disturbed largely within the last 10 years (Figure 10). A related trend is seen in Figure 11, where high flowering success sites are associated with low-to-moderate basal area. Additionally, high flowering success sites appear to have high levels of cover by shrubs (Figure 12) and high light levels (Figure 13). Recently disturbed, relatively low basal area, high 
shrub coverage, and high light sites with a diverse herb layer seem to be favorable to flowering success.

Although ordination surfaces are labeled with Crowngroups and Flowergroups, it must be remembered that the position of a site on an ordination surface is the result of the herbaceous plant community measured at each site. If the Crowngroups or Flowergroups cluster together or demonstrate a strong relationship with an ordination surface or vector, this reflects that levels of Crowngroup or Flowergroup have similar plant communities. The ordination surface with sites labeled by Crowngroup or Flowergroup and overlain by an environmental gradient allows for the visual representation of complex relationships between $T$. stoloniferum abundance or reproductive success, herbaceous plant community composition, and specific environmental variables.

\section{Multi-Response Permutation Procedure}

I found a significant global difference in herbaceous layer community composition between sites with different classifications of Crowngroup based upon the results of MRPP (Table 6). There were significant pairwise differences (with a significance level of $\mathrm{p}<0.05$ ) between groups 0 and 1,2 and 3 and a significant difference between Crowngroup 1 and Crowngroup 3 (Table 7). Crowngroups 1 and 2 do not have significantly different community compositions, nor do Crowngroups 2 and 3 . The critical value of $\mathrm{p}$ for pairwise comparisons was not adjusted for multiple comparisons. This suggests that the community composition of control sites (Crowngroup 0) was very different than those sites with T. stoloniferum present (Crowngroups 1, 2, and 3).

I also found a significant global difference in herbaceous layer community composition between sites with differerent classifications of Flowergroup (Table 8). 
Flowergroup 0 and Flowergroups 2 and 3 were significantly different from each other based upon pairwise comparisons (Table 9). There was also a significant difference in community composition between Flowergroups 2 and 3. However, there was not a significant difference between Flowergroups 1 and 3. There were also no significant differences between Flowergroups 0 and 1 and Flowergroups 1 and 2.

\section{Classification Tree Analyses}

Shannon's index of diversity (SHANNON), which I used to measure herbaceouslayer diversity, is the first variable used to split the classification tree with Crowngroup as the response, with the majority of medium-to-high $T$. stoloniferum abundance sites having a diversity index $\geq 2.53$, and the majority of low abundance or absent sites had a diversity index less than this value (Figure 14). The low abundance and absent sites were then split based on shrub coverage (SHRUBCOV), with the sites with some $T$. stoloniferum having higher shrub coverage.

Figure 15 presents the outcome of a classification tree analysis with Crowngroup as the response and Shannon's diversity index excluded from the model parameters (model structure: Crowngroup Time since last disturbance (TSLD)+ basal area (BA)+ sapling tally (SAPTALLY)+ shrub cover (SHRUBCOV)+ total herbaceous cover (TOTALVEGCOVER)+ canopy openness (OPEN)+ light density (PPFD)). Diversity of the herbaceous layer is a reflection of a myriad of factors, including forest structure, the physical structure of the herb layer, light availability, and disturbance history. By excluding this variable we can gain insight into the relative importance of environmental variables. Total herbaceous layer cover (which includes saplings and other young woody stems) was used to discriminate between control sites without $T$. 
stoloniferum and sites that contained T. stoloniferum- 4 out of 11 control sites had total vegetative cover in the herb layer less than 35.2\%. Light levels (PPFD) distinguished five sites with low abundance of $T$. stoloniferum. Sites with a longer time since last disturbance were next separated from the remaining sites. The majority of these sites were control sites, but there were 2 medium-abundance sites that had not been disturbed in the last 11.5 years. Finally, canopy openness was used to distinguish among the remaining sites. Seven out of ten Crowngroup 3 sites had canopy openness greater than $9.89 \%$, as did five of the seven remaining medium abundance (Crowngroup 2) sites. All remaining low abundance (Crowngroup 1) sites had a canopy openness less than $9.89 \%$. Time since last disturbance was the first variable used to split the classification tree with Flowergroup as the response variable (Figure 16). Sites with no $T$. stoloniferum inflorescences were characterized by having a time since last disturbance greater than 9.5 years. Photosynthetic photon flux density (PPFD) was used next to split the tree, with high flowering sites (Flowergroup 3) characterized by levels of photosynthetically active radiation greater than $8.73 \mu \mathrm{mol}$ photons $/ \mathrm{m}^{2} / \mathrm{second}$. The remaining sites, largely Flowergroups 0,1 , and 2 sites, were then split by total herbaceous cover (TOTALVEGCOVER), with the no-to-low flowering sites characterized by a mean percent cover less than $58 \%$. In general, it appears that disturbance and the resultant changes to light environment and vegetative structure are important in determining flowering success for T. stoloniferum.

Indicator Species Analysis

Symphiotrichum cordifolium and Ageratina altissima were strongly associated with high T. stoloniferum abundance sites (Crowngroup 3), and Circaea lutetiana and 
Campanula americana were associated with moderate abundance (Crowngroup 2) sites (Table 10). High flowering success sites (Flowergroup 3) were characterized by the weedy species Plantago rugelii, Prunella vulgaris, and Oxalis stricta (Table 11). These weedy species within the context of a forest road or path in addition to Amphicarpaea bracteata did appear to be good indicators of habitat suitability for T. stoloniferum.

Indicator species analysis should be interpreted with caution. Indicator species analysis revealed many species with relatively strong associations with different Crowngroups or Flowergroups. Many of the species listed as having strong relationships with a particular Crowngroup or Flowergroup could easily appear in a wide variety of habitats. Additionally, some species which from casual observation appear to be associated with $T$. stoloniferum, such as Amphicarpaea bracteata, commonly known as hog-peanut, were not found to be statistically significant.

\section{Discussion}

Trifolium stoloniferum, as suggested by its specific epithet, is readily capable of vegetative reproduction (USFWS 2007). However, I did not measure the effects of environment upon the relative investment of $T$. stoloniferum in vegetative versus sexual reproduction (this would have involved destructive sampling of plant tissues). The recovery plan for $T$. stoloniferum instructs personnel monitoring T. stoloniferum populations to count rooted crowns, and not to attempt to distinguish between physiologically-independent individuals (USFWS 2007). Thus, I used inflorescence production as a surrogate for total reproductive success in T. stoloniferum. There is literature evidence to suggest that this approach was valid. For example, Verburg \& During (1998) found that light limitation decreased production of rhizome numbers and 
weight as well as fruit number in the understory plant Circaea lutetiana, enchanter's nightshade, which was an associate of T. stoloniferum. Pitelka et al. (1980) studied the reproduction of Aster acuminatus, whorled aster, in response to patch abundance and light. They found that vigorous patches with high flowering levels and abundant individuals occurred in better lit patches. Also, they determined that larger individual plants invested more in sexual reproduction, and that investment in vegetative reproduction was relatively constant. Thus, it seems highly unlikely that a $T$. stoloniferum patch experiencing resource abundance would invest heavily in asexual reproduction without equal investment in sexual reproduction.

The response of $T$. stoloniferum to disturbance follows a pattern characteristic of perennial, summer-leaved herbs of temperate deciduous forests. Whigham (2004) concluded that most summer-leaved herbs are light-limited, and that many species demonstrate increased growth and reproductive effort when exposed to the elevated light conditions typical of small-to-moderate disturbances to the forest canopy. Shrub cover, total herbaceous cover, and herbaceous diversity were all greater in vigorously flowering and abundant patches, suggesting that the response of many members of the understory community to disturbance is similar to that of $T$. stoloniferum.

It is possible that the environmental conditions favorable to $T$. stoloniferum are to a certain extent self-perpetuating. The vigorous, dense, and diverse herbaceous vegetation that is promoted by the creation of gaps in forest can contribute to the shading of tree seedlings and suppression of tree regeneration, thus preventing shading of the herb layer and consequent herbaceous decline (Poulson \& Platt 1989, George \& Bazzaz 2003). This phenomenon is particularly well-dominated in New England forests dominated by 
ferns (George \& Bazzaz 2003) and in the southern Appalachians by the shrub species Rhododendron maximum L. and Kalmia latifolia L.. A vigorous herbaceous layer can reduce light levels below the herb layer by $70 \%$, as can the dense thickets of Rhododendron or Kalmia. Vigorous herb layers filter and delay tree regeneration; however, different tree species tolerate given conditions and survive as seeds, germinate, and grow into saplings and eventually trees when others cannot. George \& Bazzaz 1999 found that Acer rubrum L. and Fraxinus americana L. emergence were not affected by thick cover by the fern Dennstaedtia punctilobula (Michx.), while Pinus strobus, Quercus rubra, and Betula lenta emergence was reduced. These effects could not be attributed to allelopathy (see Horsley 1993). It is possible that the vigorous herb layers found around successful T. stoloniferum sites perpetuate suitable conditions by prevention of understory shading; in addition, the heavily disturbed soil and repeatedly trammeled sites in which $T$. stoloniferum grows are potentially not conducive to the regeneration of trees (see Patch 9-46 in Appendix B for an example of a site in which tree recruitment might be limited by heavy soil disturbance and a vigorous, resourcedominating herbaceous layer). However the role of periodic disturbance in stimulating reproduction and patch abundance increases indicates that the importance of the herbaceous layer as a filter in these forests is limited and insufficient to maintain suitable habitat conditions for T. stoloniferum. Additionally, in some gaps which had previously contained vigorous populations of $T$. stoloniferum, competition from tall and vigorous herbaceous competition had apparently led to T. stoloniferum decline. So, periodic disturbance to the canopy and to the ground level appears to maintain the optimum habitat conditions in which T. stoloniferum can thrive. 
The question then emerges- how did T. stoloniferum thrive before the advent of log skidders, gravel roads, and mechanical felling of trees? There is a wide base of evidence that forest conditions of the pre-Columbian period and early era of European people's influence were characterized by a much greater role of fire and importance of fire-adapted plants and ecosystems (Nowacki \& Abrams 2008). The response of $T$. stoloniferum to fire is not well characterized, but my studies have shined insight into the role of light in maintaining T. stoloniferum. Ecological processes, including fire, which maintain greater canopy openness and light penetration to the herbaceous layer will favor T. stoloniferum. Additionally, the historical and ecological evidence is strong that $T$. stoloniferum was adapted to thrive on the trampled and grazed trails and riparian corridors maintained by the megafauna of the region, including Bison bison athabascae, the woodland bison, and Cervus canadensis canadiensis, or the eastern elk. Its extant predilection for skid roads, trails, improved roads, and mowed savannahs, cemeteries, and lawns (where mowing provide a surrogate for grazing) and its inability to thrive in undisturbed habitats strongly suggest the role of large animals in maintaining suitable habitat. The once substantial impact and ecological functioning of megafauna such as woodland bison and elk in the eastern deciduous forests has disappeared (Jakle 1968), and along with massive habitat change in the industrializing of the American forests during the 19th and 20th centuries (Lewis 1968) contributed to its decline.

This species is recovering in some areas of its range (USFWS 2007), but in other areas there remain clear threats to its recovery. First, forest management practices and successional pathways that contribute to dense-shading and the recruitment of shadetolerant tree species will likely inhibit the growth of this species in the context of 
hardwood forests (Schuler 2004). "Mesophication" of the habitat of this species by dense shade-tolerant tree regeneration in the understory will negatively impact this species (Nowacki \& Abrams 2008). Secondly, the periodic disturbances upon which $T$. stoloniferum depends to create favorable habitat are often caused or influenced by human activity: logging, trampling, fire, or any other disturbance that both disturbs soil and litter and perforates the canopy. Consequently, this species could experience declines if the frequency or type of human intervention in potential habitats are unfavorable for $T$. stoloniferum. Thus, management of $T$. stoloniferum reflects but a small fragment of the implications of conservation and forest management in the Anthropocene, in which human activity must be considered an essential component of ecosystem functioning (Periman 2006).

\section{Conclusions}

I have outlined habitat conditions which promote $T$. stoloniferum success in a forested context. Success, as measured by patch abundance and flowering success, were strongly controlled by the time elapsed since most recent disturbance and light levels. Both disturbance and light interact to influence the response of the plant community, of which T. stoloniferum is a part. Successful T. stoloniferum patches are characterized by greater species diversity in the herbaceous layer, which is a result of increased light. Patches of T. stoloniferum appear inherently ephemeral, as control sites with no $T$. stoloniferum were characterized by a most recent disturbance occurring greater than 9.5 years before the study. Management that promotes T. stoloniferum success will also promote other forest herb species and contribute to the maintenance of diversity in mixed mesophytic deciduous forests. 


\section{Literature cited}

Brooks RE (1983) Trifolium stoloniferum, running buffalo clover: Description, distribution, and current status. Rhodora 85(842):343-354.

Campbell JJN, Evans M, Medley ME \& NL Taylor (1988) Buffalo clovers in Kentucky (Trifolium stoloniferum and T. reflexum): Historical records, presettlement environment, rediscovery, endangered status, cultivation and chromosome number. Rhodora 90(864):399-418.

Crawford DJ, Esselman EJ, Windus JL \& CS Pabin (1998) Genetic variation in running buffalo clover (Trifolium stoloniferum: Fabaceae) using random amplified polymorphic DNA markers (RAPDs) Annals of the Missouri Botanical Garden 85:81-89.

Daudin JJ (1986) Selection of variables in mixed-variable discriminant analysis. Biometrics 42(3):473-4

De'ath G \& KE Fabricius (2000) Classification and regression trees: A powerful yet simple technique for ecological data analysis. Ecology 81(11):3178-3192.

Delcourt HR \& PA Delcourt (1997) Pre-Columbian Native American Use of Fire on Southern Appalachian Landscapes. Conservation Biology 11(4):1010-1014.

Dufrene M \& Legendre P (1997) Species assemblages and indicator species: the need for a flexible asymmetrical approach. Ecological Monographs 67(3):345-366.

ESRI (Environmental Systems Resource Institute) (2009) ArcMap 9.2. ESRI, Redlands, California.

Ford WM, Madarish D \& TM Schuler (2003) Influence of white-tailed deer digestion on running buffalo clover (Trifolium stoloniferum: Fabaceae Muhl. ex. A. Eaton). American Midland Naturalist 149:425-428.

Franklin CJ (1998) Self-compatibility and variation in seed production among Ohio populations of federally endangered Trifolium stoloniferum (Fabaceae). M.S. Thesis. The Ohio State University, Columbus, OH.

George LO \& FA Bazzaz (2003) The herbaceous layer as a filter determining spatial pattern in forest tree regeneration. Pgs. 265-282 in The Herbaceous Layer in Forests of Eastern North America edited by FS Gilliam \& MR Roberts, Oxford University Press, New York, NY.

George LO \& FA Bazzazz (1999b) The fern understory as an ecological filter: growth and survival of canopy tree seedlings. Ecology 80:846-856. 
Hattenbach MJ (1996) Edaphic relations of an endangered plant, Trifolium stoloniferum Muhl. ex. A. Eaton. M.S. Thesis. The Ohio State University, Columbus, OH.

Horsley SB (1993) Role of allelopathy in hayscented fern interference with Allegheny hardwood production. Journal of Chemical Ecology 19:2737-2755.

Jakle JA (1968) The American bison and the human occupance of the Ohio Valley. Proceedings of the American Philosophical Society. 112(4):299-305.

Lewis RL (1998) Transforming the Appalachian countryside: Railroads, deforestation, and social change in West Virginia, 1880-1920. The University of North Carolina Press, Chapel Hill, NC.

MacNally R (2000) Regression and model-building in conservation biology, biogeography, and ecology: The distinction between- and reconciliation of'predictive' and 'explanatory' models. Biodiversity and Conservation 9(5):655671.

MacNally R (2002) Multiple regression and inference in ecology and conservation biology: further comments on identifying important predictor variables. Biodiversity and Conservation 11(8):1397-1401.

Madarish DM, Rodrigue JL \& MB Adams (2002) Vascular flora and macroscopic fauna on the Fernow Experimental Forest. USDA Forest Service Northeastern Research Station General Technical Report NE-291.

Madarish D \& TM Schuler (2002) Effects of forest management practices on the federally endangered running buffalo clover (Trifolium stoloniferum Muhl. ex. A. Eaton). Natural Areas Journal 22(2):120-128.

McCune B \& JB Grace (2002) Analysis of Ecological Communities. MJM Software Design, Gleneden Beach, Oregon, USA.

McCune B \& MJ Mefford (2006) PC-ORD. Multivariate Analysis of Ecological Data. Version 5. MjM Software, Gleneden Beach, Oregon, USA.

Morris DR, Baligar VC, Schuler TM \& PJ Harmon (2002) Biological nitrogen fixation and habitat of running buffalo clover. Journal of Plant Nutrition 25(4): 735-746.

Nowacki GJ \& MD Abrams (2008) The demise of fire and mesophication of forests in the eastern United States. Bioscience 58(2):123-138.

Oksanen J (2010) envfit: Fits an environmental vector or factor onto an ordination. R Documentation $<$ http://127.0.0.1:29523/library/vegan/html/envfit.html $>$. Accessed 16 July 2010. 
Peet RK, Wentworth TR \& PS White (1998) A flexible, multipurpose method for recording vegetation composition and structure. Castanea 63(3):262-274.

Periman RD (2006) Visualizing the Anthropocene: Human land-use history and environmental management.

Pitelka LF, Stanton DS \& MO Peckenham (1980) Effects of light and density on resource allocation in a forest herb, Aster Acuminatus (Compositae). American Journal of Botany 67(6):942-948.

Poulson TL \& WJ Platt (1989) Gap light regimes influence canopy tree diversity. Ecology 70(3):553-555.

Regent Instruments Inc. (2006) Winscanopy system version 2006 b.

R Development Core Team (2009) R: A language and environment for statistical computing. R Foundation for Statistical Computing, Vienna, Austria. ISBN 3900051-07-0, URL http://www.R-project.org.

Schuler TM (2004) Fifty years of partial harvesting in a mixed mesophytic forest: composition and productivity. Canadian Journal of Forest Research 34:985-997.

Taylor, N.L., J.M. Gillett, J.J.N. Campbell, and S. Berger. 1994. Crossing and morphological relationships among native clovers of eastern North America. Crop Science 34(4): 1097-1100.

Tear TH, Kareiva P, Angermeier PL, Comer P, Czech B, Kautz R, Landon L, Mehlman D, Murphy K, Ruckelshaus M, Scott JM \& Wilhere G (2005) How much is enough? The recurrent problem of setting measurable objectives in conservation. BioScience 55(10):835-849.

USFWS (2007) Running buffalo clover (Trifolium stoloniferum) Recovery Plan: First revision. United States Fish \& Wildlife Service, Fort Snelling, MN.

Verburg RW \& HJ During (1998) Vegetative propagation and sexual reproduction in the woodland understory pseudo-annual Circaea lutetiana 134(2):211-224.

White PS (1979) Pattern, process and natural disturbance in vegetation. Botanical Review 45(3):229-299. 


\section{Tables}

Table 1. Means and standard deviations of environmental variables by Crowngroup $(0=$ no rooted crowns, $1=1-30$ rooted crowns, $2=31-99$ rooted crowns, and 3 $=>100$ rooted crowns). Note: Environmental variables: Slope $=\%$ slope; Aspect $=$ raw aspect measurement (not cosine transformed); TSLD $=$ Time Since Last Disturbance (years); BA=Basal Area (sq. ft./ac.); Gravel, CWD (Coarse Woody Debris), Litter (leaf litter), MINSOIL (exposed mineral soil), Moss, Rock=mean \% cover in 5 subplots; LeafDepRoad = mean depth $(\mathrm{cm})$ of leaf litter in 3 road subplots; LeafFor $=$ depth $(\mathrm{cm})$ of leaf litter in forest subplot; LeafRM= depth $(\mathrm{cm})$ of leaf litter in road margin subplot; SAPTALLY= sapling tally for $100 \mathrm{~m}^{2}$ plot; SHRUBCOV= Distance of shrub cover (meters) recorded along two transects in each $100 \mathrm{~m}^{2}$ subplot; TOTVEGCOVER $=$ Mean vegetative cover (\% cover) in 5 subplots; GAPFRAC $=$ Gap fraction, or the chance of not hitting a leaf when casting a ray of light downwards; OPEN= Canopy openness, in \% open sky; PPFD= Photosynthetic photon flux density, in $\mu \mathrm{mol}$ photons $/ \mathrm{m}^{2} /$ second; and SHANNON= Shannon's diversity index, which is a measure that two randomly chosen individuals

(or units of percent cover) will come from different species.

\begin{tabular}{|c|c|c|c|c|c|c|c|c|c|c|c|c|c|}
\hline Crowngroup & $\mathrm{N}$ & Slope & SD & Aspect & SD & TSLD & SD & BA & SD & GRAVEL & SD & CWD & SD \\
\hline $\mathbf{0}$ & 11 & 27.64 & 12.33 & 313.36 & 24.77 & 19.64 & 19.10 & 111.8 & 34.9 & 0.00 & 0.00 & 5.38 & 6.89 \\
\hline 1 & 12 & 28.17 & 16.68 & 246.92 & 106.54 & 9.83 & 11.26 & 80.8 & 26.1 & 0.81 & 2.79 & 1.50 & 2.05 \\
\hline 2 & 9 & 29.11 & 13.72 & 274.89 & 65.91 & 8.88 & 10.60 & 94.4 & 39.1 & 0.00 & 0.00 & 2.11 & 2.78 \\
\hline 3 & 10 & 30.30 & 12.60 & 289.30 & 42.57 & 4.8 & 3.08 & 104 & 49.5 & 4.73 & 14.97 & 3.37 & 5.91 \\
\hline Overall & 42 & 28.74 & 13.58 & 280.40 & 71.32 & 11 & 13.33 & 97.4 & 38.3 & 1.36 & 7.42 & 3.09 & 4.93 \\
\hline Crowngroup & $\mathrm{N}$ & Litter & SD & MINSOIL & SD & Moss & SD & Rock & SD & LeafDepRoad & SD & LeafFor & SD \\
\hline $\mathbf{0}$ & 11 & 74.74 & 31.60 & 17.06 & 24.91 & 1.91 & 3.67 & 0.91 & 1.26 & 1.01 & 0.78 & 0.83 & 0.67 \\
\hline 1 & 12 & 80.00 & 19.74 & 15.56 & 19.43 & 1.28 & 2.67 & 0.86 & 0.85 & 1.22 & 0.57 & 1.07 & 0.38 \\
\hline 2 & 9 & 86.82 & 10.62 & 6.59 & 9.48 & 0.48 & 1.12 & 4.11 & 8.93 & 1.26 & 0.63 & 1.07 & 0.58 \\
\hline 3 & 10 & 75.20 & 28.58 & 13.80 & 13.13 & 1.27 & 2.28 & 1.63 & 3.59 & 1.17 & 0.56 & 0.90 & 0.53 \\
\hline Overall & 42 & 78.94 & 23.91 & 13.61 & 17.98 & 1.27 & 2.61 & 1.75 & 4.54 & 1.16 & 0.63 & 0.96 & 0.54 \\
\hline Crowngroup & $\mathrm{N}$ & LeafRM & SD & SAPTALLY & SD & SHRUBCOV & SD & TOTVEGCOVER & $\mathrm{SD}$ & GAPFRAC & $\mathrm{SD}$ & OPEN & SD \\
\hline $\mathbf{0}$ & 11 & 0.75 & 0.49 & 22.64 & 32.79 & 1.89 & 2.93 & 47.60 & 20.57 & 10.61 & 5.98 & 9.13 & 6.11 \\
\hline 1 & 12 & 0.76 & 0.36 & 9.83 & 8.36 & 2.46 & 1.79 & 62.82 & 14.77 & 9.42 & 2.79 & 10.08 & 3.06 \\
\hline 2 & 9 & 0.88 & 0.38 & 24.56 & 15.08 & 3.46 & 4.66 & 64.86 & 18.24 & 9.81 & 3.13 & 9.53 & 3.98 \\
\hline 3 & 10 & 0.89 & 0.42 & 13.80 & 14.23 & 3.71 & 3.70 & 66.29 & 13.37 & 9.12 & 3.26 & 9.55 & 3.30 \\
\hline Overall & 42 & 0.81 & 0.41 & 17.29 & 20.21 & 2.82 & 3.28 & 60.09 & 18.01 & 9.74 & 3.92 & 9.58 & 4.15 \\
\hline Crowngroup & $\mathrm{N}$ & LAI & $\mathrm{SD}$ & PPFD & $\mathrm{SD}$ & SHANNON & $\mathrm{SD}$ & & & & & & \\
\hline 0 & 11 & 4.35 & 1.47 & 7.48 & 3.90 & 2.38 & 0.35 & & & & & & \\
\hline 1 & 12 & 4.26 & 1.25 & 7.63 & 5.70 & 2.63 & 0.27 & & & & & & \\
\hline 2 & 9 & 4.29 & 1.60 & 8.64 & 3.17 & 2.97 & 0.19 & & & & & & \\
\hline 3 & 10 & 4.22 & 1.04 & 9.53 & 3.60 & 2.77 & 0.35 & & & & & & \\
\hline Overall & 42 & 4.28 & 1.30 & 8.26 & 4.24 & 2.67 & 0.36 & & & & & & \\
\hline
\end{tabular}


Table 2. Means and standard deviations of environmental variables by Flowergroup $(0=$ no flowers, $1=0.01-0.05$ flowering index, $2=0.06-$ $0.19 \mathrm{FI}$, and 3=>0.20 FI). Environmental variables: Slope $=\%$ slope; Aspect $=$ raw aspect measurement (not cosine transformed); TSLD= Time Since Last Disturbance (years); BA=Basal Area (sq. ft./ac.); Gravel, CWD (Coarse Woody Debris), Litter (leaf litter), MINSOIL (exposed mineral soil), Moss, Rock=mean \% cover in 5 subplots; LeafDepRoad= mean depth (cm) of leaf litter in 3 road subplots; LeafFor= depth $(\mathrm{cm})$ of leaf litter in forest subplot; LeafRM $=$ depth $(\mathrm{cm})$ of leaf litter in road margin subplot; SAPTALLY= sapling tally for $100 \mathrm{~m}^{2}$ plot; SHRUBCOV= Distance of shrub cover (meters) recorded along two transects in each $100 \mathrm{~m}^{2}$ subplot; TOTVEGCOVER= Mean vegetative cover (\% cover) in 5 subplots; GAPFRAC = Gap fraction, or the chance of not hitting a leaf when casting a ray of light downwards; OPEN= Canopy openness, in \% open sky; PPFD= Photosynthetic photon flux density ( $\mu \mathrm{mol}$ photons $\left./ \mathrm{m}^{2} / \mathrm{second}\right)$; and SHANNON= Shannon's diversity index, which is a measure that two randomly chosen individuals (or units of percent cover) will come from different species.

\begin{tabular}{|c|c|c|c|c|c|c|c|c|c|c|c|c|c|}
\hline Flowergroup & $\mathrm{N}$ & Slope & SD & Aspect & SD & TSLD & SD & $\mathrm{BA}$ & $\mathrm{SD}$ & GRAVEL & $\mathrm{SD}$ & CWD & $\mathrm{SD}$ \\
\hline 0 & 17 & 27.18 & 12.70 & 293.88 & 54.68 & 18.88 & 16.87 & 110.6 & 30.5 & 0.00 & 0 & 4.17 & 5.96 \\
\hline 1 & 5 & 30.40 & 13.79 & 253.60 & 76.47 & 10.20 & 13.10 & 96 & 55 & 0.00 & 0 & 2.07 & 2.13 \\
\hline 2 & 8 & 27.25 & 12.26 & 286.75 & 80.46 & 4.50 & 2.73 & 81.3 & 18.9 & 0.00 & 0 & 1.21 & 1.02 \\
\hline 3 & 12 & 31.25 & 14.47 & 268.25 & 86.97 & 4.50 & 2.88 & 90 & 47.5 & 4.75 & 13.70 & 3.25 & 5.62 \\
\hline Overall & 42 & 28.74 & 13.41 & 280.40 & 71.32 & 13.36 & 13.33 & 97.4 & 38.3 & 1.36 & 7.42 & 3.09 & 4.93 \\
\hline Flowergroup & $\mathrm{N}$ & Litter & SD & MINSOIL & SD & Moss & SD & Rock & SD & LeafDepRoad & SD & LeafFor & $\mathrm{SD}$ \\
\hline 0 & 17 & 78.15 & 26.61 & 15.47 & 21.73 & 1.53 & 3.06 & 0.69 & 1.06 & 1.02 & 0.70 & 0.93 & 0.59 \\
\hline 1 & 5 & 86.47 & 12.54 & 10.07 & 12.45 & 1.13 & 1.59 & 0.27 & 0.60 & 1.54 & 0.23 & 1.46 & 0.24 \\
\hline 2 & 8 & 83.55 & 17.88 & 12.83 & 14.94 & 2.13 & 3.76 & 0.42 & 0.53 & 1.11 & 0.62 & 0.85 & 0.57 \\
\hline 3 & 12 & 73.86 & 27.73 & 12.97 & 17.53 & 0.39 & 0.62 & 4.78 & 7.80 & 1.23 & 0.62 & 0.88 & 0.45 \\
\hline Overall & 42 & 78.94 & 23.91 & 13.61 & 17.98 & 1.27 & 2.61 & 1.75 & 4.54 & 1.16 & 0.63 & 0.96 & 0.54 \\
\hline Flowergroup & $\mathrm{N}$ & LeafRM & SD & SAPTALLY & SD & SHRUBCOV & SD & TOTVEGCOVER & $\mathrm{SD}$ & GAPFRAC & SD & OPEN & SD \\
\hline $\begin{array}{ll}0 & 0 \\
0 & 0\end{array}$ & 17 & 78.15 & 26.61 & 15.47 & 21.73 & 1.53 & 3.06 & 0.69 & 1.06 & 1.02 & 0.70 & 0.93 & 0.59 \\
\hline 1 & 5 & 86.47 & 12.54 & 10.07 & 12.45 & 1.13 & 1.59 & 0.27 & 0.60 & 1.54 & 0.23 & 1.46 & 0.24 \\
\hline 2 & 8 & 83.55 & 17.88 & 12.83 & 14.94 & 2.13 & 3.76 & 0.42 & 0.53 & 1.11 & 0.62 & 0.85 & 0.57 \\
\hline 3 & 12 & 73.86 & 27.73 & 12.97 & 17.53 & 0.39 & 0.62 & 4.78 & 7.80 & 1.23 & 0.62 & 0.88 & 0.45 \\
\hline Overall & 42 & 78.94 & 23.91 & 13.61 & 17.98 & 1.27 & 2.61 & 1.75 & 4.54 & 1.16 & 0.63 & 0.96 & 0.54 \\
\hline Flowergroup & $\mathrm{N}$ & LAI & SD & PPFD & SD & SHANNON & SD & & & & & & \\
\hline 0 & 17 & 4.27 & 1.37 & 6.54 & 3.66 & 2.48 & 0.36 & & & & & & \\
\hline 1 & 5 & 4.31 & 0.77 & 6.38 & 2.12 & 2.66 & 0.23 & & & & & & \\
\hline 2 & 8 & 4.05 & 1.45 & 7.28 & 3.05 & 2.88 & 0.27 & & & & & & \\
\hline 3 & 12 & 4.43 & 1.40 & 12.14 & 4.07 & 2.79 & 0.35 & & & & & & \\
\hline Overall & 42 & 4.28 & 1.30 & 8.26 & 4.24 & 2.67 & 0.36 & & & & & & \\
\hline
\end{tabular}


Table 3. Comparison between control sites that used to contain T. stoloniferum and sites that never contained T. stoloniferum. No clear patterns emerge to distinguish these two types of sites from one another.

\begin{tabular}{|c|c|c|c|c|c|c|c|c|}
\hline & TSLD & SD & $\mathrm{BA}$ & SD & SAPTALLY & SD & SHRUBCOV & $\mathrm{SD}$ \\
\hline $\begin{array}{l}\text { Used to } \\
\text { contain }\end{array}$ & 21.0 & 24.4 & 9.3 & 3.1 & 14.5 & 12.7 & 1.3 & 2.1 \\
\hline $\begin{array}{l}\text { Never } \\
\text { contained }\end{array}$ & 18.9 & 17.6 & 12.3 & 3.4 & 27.3 & 40.5 & 2.2 & 3.4 \\
\hline & TOTVEGCOVER & SD & OPEN & SD & PPFD & SD & & \\
\hline $\begin{array}{l}\text { Used to } \\
\text { contain }\end{array}$ & 48.8 & 33.2 & 10.1 & 2.9 & 7.0 & 3.7 & & \\
\hline $\begin{array}{l}\text { Never } \\
\text { contained }\end{array}$ & 46.9 & 12.3 & 8.6 & 7.6 & 7.8 & 4.3 & & \\
\hline
\end{tabular}

Table 4a and b. Results of MANOVA analyses performed with Crowngroup and Flowergroup as predictive factor and collective environmental variables as the multivariate response.

a.

a.
\begin{tabular}{|l|l|l|l|l|l|l|}
\hline & Df & Pillai & approx F & num Df & den Df & $\operatorname{Pr}(>\mathrm{F})$ \\
\hline Crowngroup & 3 & 1.0790 & 1.9972 & 27 & 96 & 0.008 \\
\hline Residuals & 38 & & & & & \\
\hline
\end{tabular}

b.

\begin{tabular}{|l|l|l|l|l|l|l|}
\hline & Df & Pillai & approx F & num Df & den Df & $\operatorname{Pr}(>$ F $)$ \\
\hline Flowergroup & 3 & 0.22285 & 2.1967 & 27 & 88 & 0.03 \\
\hline Residuals & 38 & & & & & \\
\hline
\end{tabular}


Table 5. Results of environmental fitting function performed on nonmetric multidimensional scaling ordination of herbaceous communities measured in T. stoloniferum and control sites.

\begin{tabular}{|l|c|c|c|c|}
\hline & NMDS1 & NMDS2 & $\mathrm{r} 2$ & $\mathrm{p}=\operatorname{Pr}(>\mathrm{r})$ \\
\hline Crowngroup & -0.17 & -0.98 & 0.18 & 0.033 \\
\hline Flowergroup & -0.43 & -0.9 & 0.39 & 0.0001 \\
\hline Aspect & 0.57 & -0.82 & 0.037 & 0.50 \\
\hline TSLD & 0.94 & 0.34 & 0.51 & 0.0001 \\
\hline BA & 0.55 & 0.84 & 0.46 & 0.0001 \\
\hline SAPTALLY & -0.92 & -0.38 & 0.16 & 0.042 \\
\hline SHRUBCOV & -0.68 & 0.73 & 0.31 & 0.002 \\
\hline TOTALVEGCOVER & 0.01 & 1.00 & 1.05 & 0.11 \\
\hline OPEN & -1.00 & -0.085 & 0.14 & 0.055 \\
\hline PPFD & -0.72 & -0.69 & 0.044 & 0.41 \\
\hline SHANNON & -0.34 & -0.94 & 0.44 & 0.0001 \\
\hline P values based on 1000 permutations. \\
\hline
\end{tabular}


Table 6. Summary statistics of multi-response permutation procedure (MRPP) with Crowngroup as the a priori grouping.

\begin{tabular}{|c|c|c|c|c|c|}
\hline Crowngroup & Number in group & $\begin{array}{c}\text { Observed Sorenson } \\
\text { distance }\end{array}$ & Test statistic T & $\begin{array}{c}\text { Chance-corrected } \\
\text { within-group } \\
\text { agreement A }\end{array}$ & P \\
\hline 0 & 11 & 0.64 & -3.07 & 0.089 & 0.0032 \\
\hline 1 & 12 & 0.41 & & & \\
\hline 2 & 9 & 0.43 & & & \\
\hline 3 & 10 & 0.33 & & & \\
\hline
\end{tabular}

Table 7. Pairwise comparisons of Crowngroups based upon findings of MRPP. P-values have been adjusted for multiple comparisons.

\begin{tabular}{|l|c|c|c|}
\hline Pairwise comparisons (Crowngroups) & $\mathrm{T}$ & $\mathrm{A}$ & $\mathrm{P}$ \\
\hline 1 vs. 0 & -2.48 & 0.079 & 0.017 \\
\hline 1 vs. 2 & 0.512 & -0.019 & 0.067 \\
\hline 1 vs. 3 & -2.074 & 0.068 & 0.0311 \\
\hline 0 vs. 2 & -2.77 & 0.094 & 0.0072 \\
\hline 0 vs. 3 & -3.24 & 0.10 & 0.0043 \\
\hline 2 vs. 3 & -0.0092 & 0.00031 & 0.46 \\
\hline
\end{tabular}


Table 8. Summary statistics of multi-response permutation procedure (MRPP) with Flowergroup as the a priori grouping.

\begin{tabular}{|c|c|c|c|c|c|}
\hline Flowergroup & Number in group & $\begin{array}{c}\text { Observed Sorenson } \\
\text { distance }\end{array}$ & Test statistic T & $\begin{array}{c}\text { Chance-corrected within- } \\
\text { group agreement A }\end{array}$ & P \\
\hline 0 & 17 & 0.56 & -4.20 & & 0.12 \\
\hline 1 & 5 & 0.47 & & & 0.0018 \\
\hline 2 & 8 & 0.30 & & & \\
\hline 3 & 12 & 0.34 & & & \\
\hline
\end{tabular}

Table 9. Pairwise comparisons of Flowergroups based upon findings of MRPP. The p-values have not been corrected for multiple comparisons.

\begin{tabular}{|c|c|c|c|}
\hline Pairwise comparisons (Flowergroups) & $\mathrm{T}$ & $\mathrm{A}$ & $\mathrm{P}$ \\
\hline 1 vs. 0 & 0.19 & -0.0060 & 0.54 \\
\hline 1 vs. 2 & -0.49 & -0.022 & 0.29 \\
\hline 1 vs. 3 & -0.052 & 0.024 & 0.45 \\
\hline 0 vs. 2 & -4.78 & 0.14 & 0.00016 \\
\hline 0 vs. 3 & -4.95 & 0.12 & 0.00013 \\
\hline 2 vs. 3 & -1.195 & 0.12 & 0.036 \\
\hline
\end{tabular}


Table 10. Results of indicator species analysis for Crowngroup. Maximum expected indicator value (IV) is based upon the random assignment of species cover to different sites, as is the standard deviation associated with this statistic. 4999 permutations were performed. The $\mathrm{p}$-value is calculated by comparing observed maximum IV to expected maximum IV and indicates the likelihood of obtaining the result by chance alone $(*=\mathrm{p}<0.05)$.

\begin{tabular}{|c|c|c|c|c|c|c|c|c|}
\hline & & \multicolumn{4}{|c|}{ Observed Indicator Values } & \multirow[b]{2}{*}{$\begin{array}{l}\text { Maximum } \\
\text { expected IV }\end{array}$} & \multirow[b]{2}{*}{ SD } & \multirow[b]{2}{*}{$\mathrm{P}$} \\
\hline Crowngroup/Species & $\begin{array}{l}\text { Number of } \\
\text { sites } \\
\text { observed }\end{array}$ & $\begin{array}{l}\text { Crowngroup } \\
0\end{array}$ & Crowngroup 1 & Crowngroup 2 & Crowngroup 3 & & & \\
\hline \multicolumn{9}{|l|}{ Crowngoup 0} \\
\hline Dioscorea quaternata & 3 & 23 & 0 & 0 & 2 & 11.4 & 6.09 & 0.061 \\
\hline \multicolumn{9}{|l|}{ Crowngroup 1} \\
\hline Grass & 30 & 4 & 47 & 23 & 23 & 34.2 & 5.79 & $0.031 *$ \\
\hline Oxalis stricta & 14 & 0 & 40 & 20 & 14 & 26.2 & 5.77 & $0.024 *$ \\
\hline \multicolumn{9}{|l|}{ Crowngroup 2} \\
\hline Barbarea vulgaris & 3 & 0 & 1 & 19 & 0 & 10.4 & 6.10 & 0.071 \\
\hline $\begin{array}{l}\text { Campanula } \\
\text { americana }\end{array}$ & 3 & 0 & 2 & 44 & 0 & 12.3 & 6.44 & $0.0010^{*}$ \\
\hline Circaea lutetiana & 10 & 2 & 13 & 38 & 17 & 24.8 & 5.74 & $0.029 *$ \\
\hline Fraxinus americana & 12 & 2 & 3 & 52 & 23 & 26.8 & 6.31 & $0.0020^{*}$ \\
\hline Rumex obtusifolium & 8 & 0 & 5 & 29 & 2 & 16.1 & 7.04 & 0.0634 \\
\hline \multicolumn{9}{|l|}{ Crowngroup 3} \\
\hline $\begin{array}{l}\text { Symphiotrichum } \\
\text { cordifolium }\end{array}$ & 14 & 0 & 2 & 22 & 35 & 20.9 & 6.7 & $0.039 *$ \\
\hline $\begin{array}{l}\text { Ageratina altissima } \\
\text { var. altissima }\end{array}$ & 29 & 10 & 21 & 22 & 38 & 29.6 & 4.06 & $0.043^{*}$ \\
\hline Hystrix patula & 2 & 0 & 0 & 0 & 20 & 10.3 & 4.48 & 0.091 \\
\hline Rubus occidentalis & 5 & 0 & 1 & 0 & 37 & 13.6 & 7.04 & 0.0068 \\
\hline
\end{tabular}


Table 11. Results of indicator species analysis for Flowergroup. Maximum expected indicator value (IV) is based upon the random assignment of species cover to different sites, as is the standard deviation associated with this statistic. 4999 permutations were performed. The $p$-value is calculated by comparing observed maximum IV to expected maximum IV and indicates the likelihood of obtaining the result by chance alone $(*=\mathrm{p}<0.05)$.

\begin{tabular}{|c|c|c|c|c|c|c|c|c|}
\hline & & \multicolumn{4}{|c|}{ Observed Indicator Values } & & & \\
\hline Flowergroup/Species & $\begin{array}{l}\text { Number of } \\
\text { sites } \\
\text { observed }\end{array}$ & Flowergroup 0 & Flowergroup 1 & Flowergroup 2 & Flowergroup 3 & $\begin{array}{l}\text { Maximum } \\
\text { expected } \\
\text { IV }\end{array}$ & SD & $\mathrm{P}$ \\
\hline \multicolumn{9}{|l|}{ Flowergroup 0} \\
\hline Eurybia divaricata & 5 & 30 & 0 & 0 & 2 & 16.3 & 8.00 & 0.061 \\
\hline Hydrophyllum canadense & 10 & 34 & 0 & 4 & 3 & 20.5 & 8.18 & 0.065 \\
\hline \multicolumn{9}{|l|}{ Flowergroup 1} \\
\hline Cryptotaenia canadensis & 22 & 27 & 40 & 9 & 13 & 31.4 & 5.52 & 0.0726 \\
\hline \multicolumn{9}{|l|}{ Flowergroup 2} \\
\hline Acer saccharum & 10 & 12 & 15 & 45 & 2 & 26.4 & 7.04 & $0.0208^{*}$ \\
\hline Arctium minus & 4 & 0 & 0 & 31 & 1 & 12.8 & 7.16 & $0.042 *$ \\
\hline Polygonum sagittatum & 3 & 0 & 0 & 24 & 0 & 12.0 & 6.76 & 0.062 \\
\hline Rubus allegheniensis & 15 & 2 & 15 & 42 & 16 & 27.3 & 8.21 & 0.059 \\
\hline Symphiotrichum cordifolium & 14 & 1 & 16 & 37 & 5 & 22.1 & 7.86 & 0.056 \\
\hline Circaea lutetiana & 10 & 4 & 4 & 39 & 25 & 25.9 & 6.63 & $0.050 *$ \\
\hline Lobelia siphilitica & 3 & 0 & 2 & 22 & 0 & 12.4 & 6.89 & 0.09 \\
\hline Unknown 1 & 8 & 13 & 6 & 36 & 1 & 21.5 & 7.43 & $0.046^{*}$ \\
\hline Vitis aestivalis & 5 & 0 & 0 & 63 & 0 & 14.0 & 7.85 & $0.0004 *$ \\
\hline \multicolumn{9}{|l|}{ Flowergroup 3} \\
\hline Liriodendron tulipifera & 8 & 4 & 0 & 10 & 32 & 20.8 & 8.13 & 0.092 \\
\hline Oxalis stricta & 14 & 1 & 12 & 28 & 41 & 27.5 & 6.81 & 0.052 \\
\hline Plantago rugelii & 11 & 1 & 2 & 14 & 57 & 24.2 & 7.51 & $0.0026^{*}$ \\
\hline Prunella vulgaris & 6 & 0 & 0 & 12 & 35 & 17.9 & 8.54 & $0.043^{*}$ \\
\hline
\end{tabular}




\section{Figures}

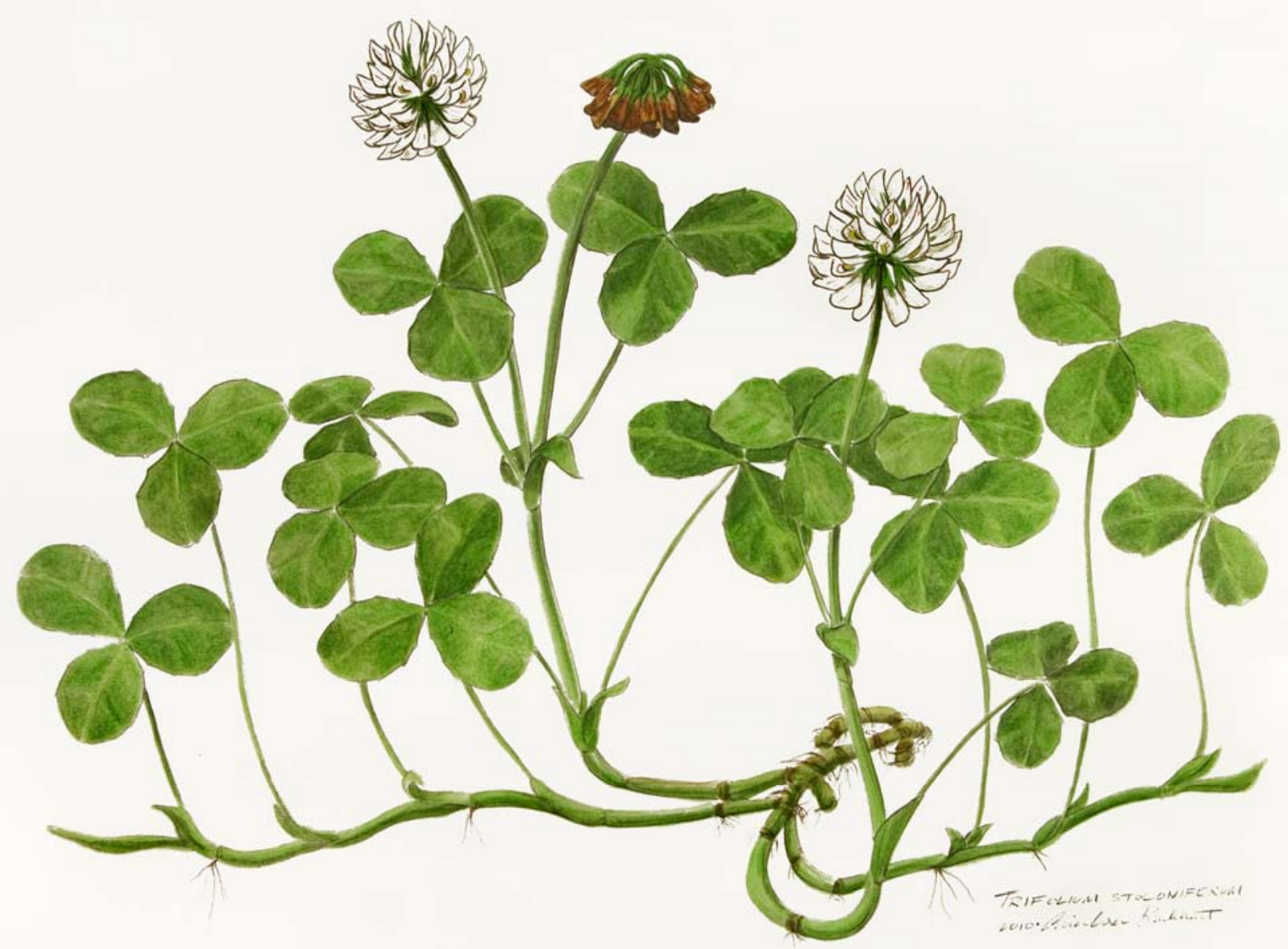

Figure 1. Illustration depicting T. stoloniferum. Trifolium stoloniferum is distinguished from other clover species by the paired leaves below the inflorescences, stoloniferous habit with rooting at the nodes, small tooths along leaflet margin. 


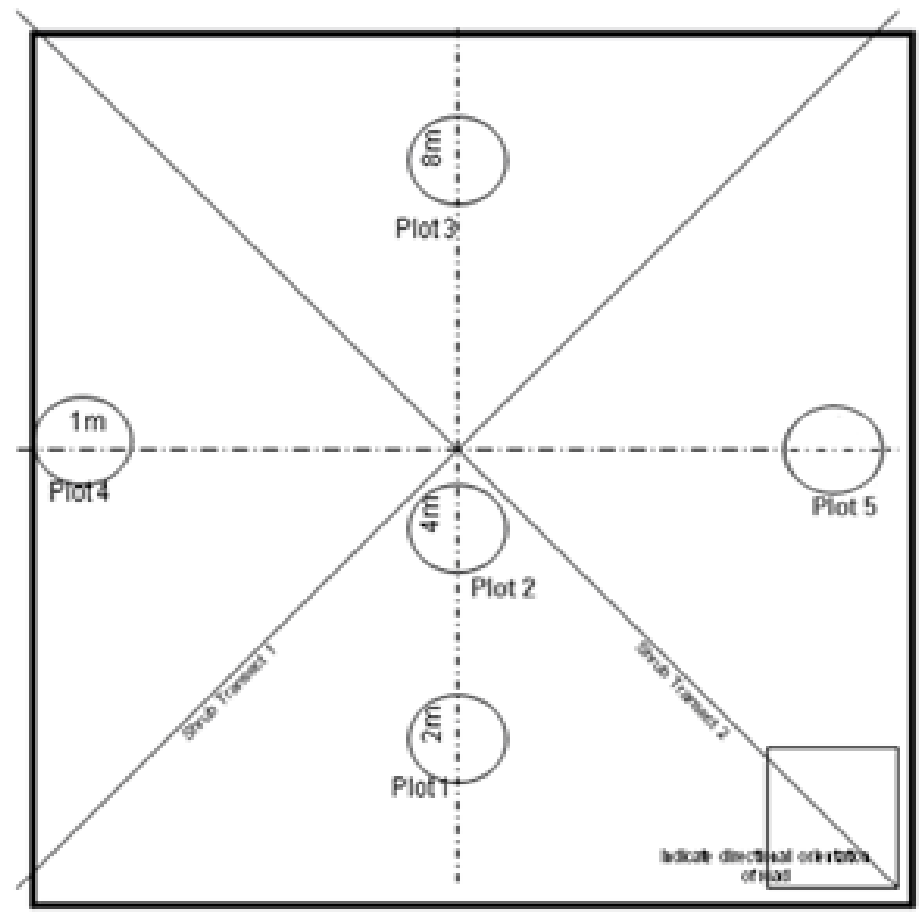

Figure 2. Habitat assessment plot design. Five $1 \mathrm{~m}^{2}$ circular subplots were located within the $100 \mathrm{~m}^{2}$ square plot. Three of the subplots (Plots 1-3 in the figure) were positioned through the center of the skid road or foot path (along wider improved roads, I positioned the plots more towards the edge of the road with the goal of sampling both on-road and off-road vegetation). Subplot 4 was positioned $4 \mathrm{~m}$ on the downhill side of the plot - the purpose of this subplot was to sample road margin vegetation. Subplot 5 was variable in its position, but was located on the uphill side of the forest and I attempted to position this plot in intact forest where the soil remained undisturbed. This was possible for nearly all plots positioned along skid roads. On improved roads, subplot 5 usually was positioned in the middle of the improved road. Two shrub transects were conducted along the diagonals of the $100 \mathrm{~m}^{2}$ plot. The number of saplings occurring in the entire $100 \mathrm{~m}^{2}$ plot was recorded. Basal area of trees in and around the plot was assessed from the exact center of the plot using a 10 basal area factor prism (U.S. units-sq. ft./acre). Slope and aspect were also assessed from the plot. 


\section{Vector overlay with Crowngroups as labels}

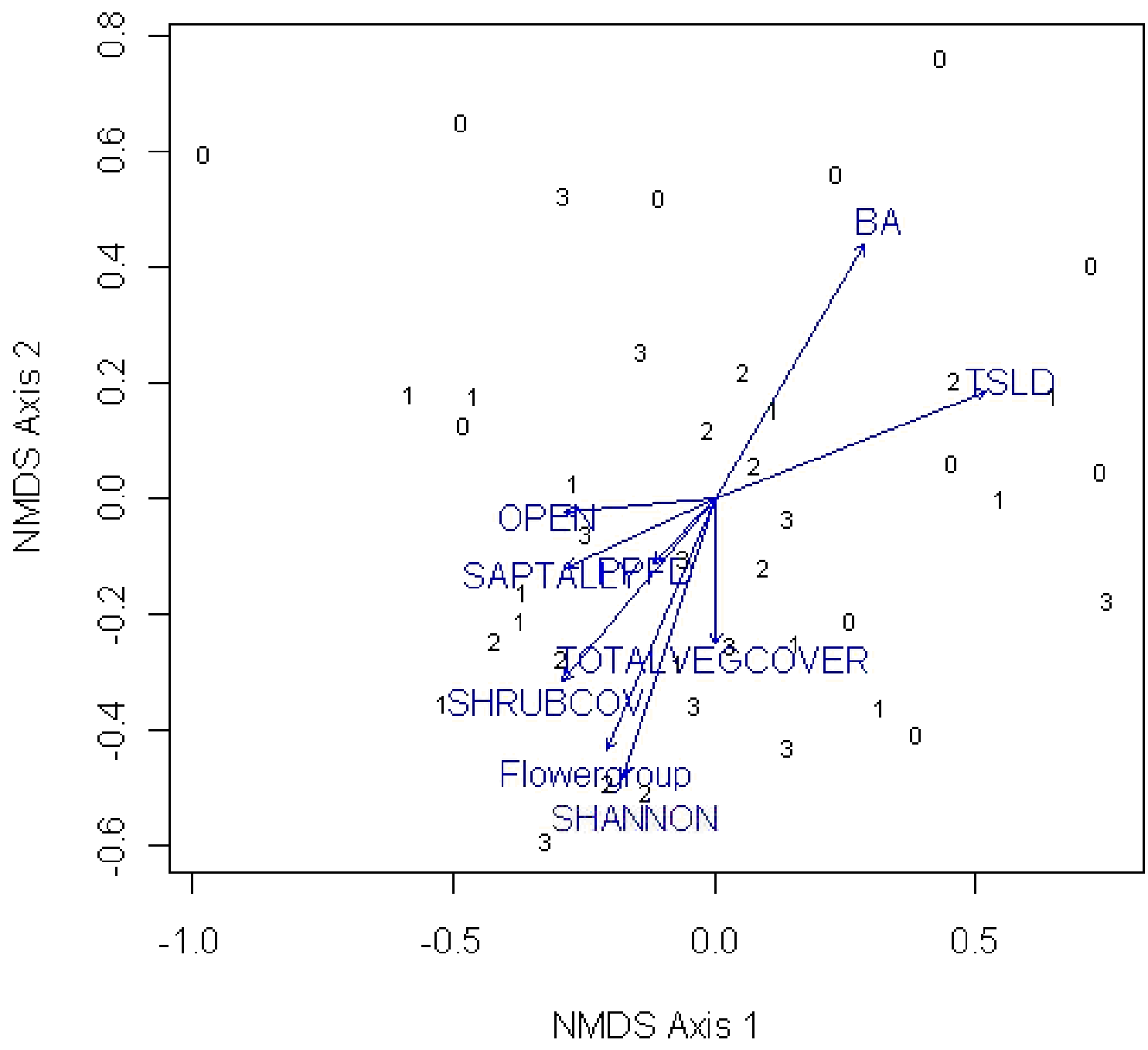

Figure 3. Crowngroups as labels: Environmetal variables as vectors. Vector direction indicates positive correlation and the length of the vector is proportional to the strength of that correlation. Crowngroup $0=0 \mathrm{~T}$. stoloniferum rooted crowns; Crowngroup 1=1-30 rooted crowns; Crowngroup 2=31-99 rooted crown; Crowngroup $3 \geq 100$ rooted crowns. 


\section{Vector overlay with Flowergroups as labels}

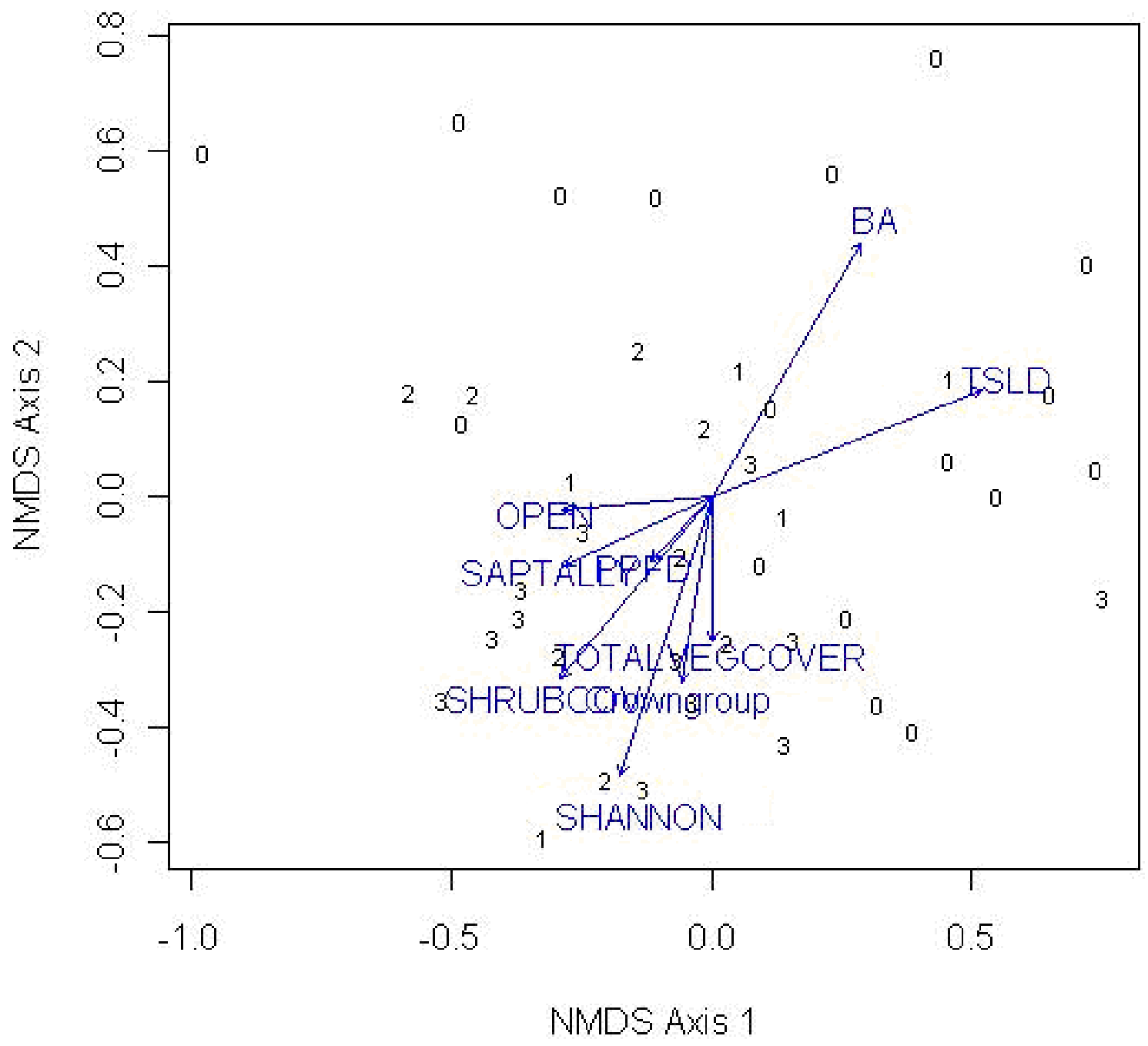

Figure 4. Flowergroups as labels: Environmental variables as vectors. Flowergroups are determined by flowering index, a measure of relative reproductive success/patch. It is measured as number of inflorescences in a patch/number of rooted crowns. Flowergroup $0=$ no flowers; Flowergroup 1=0.01-0.05 flowering index; Flowergroup 2=0.06-0.19 flowering index; and Flowergroup $3 \geq 0.20$ flowering index. 
Time Since Last Disturbance (years)

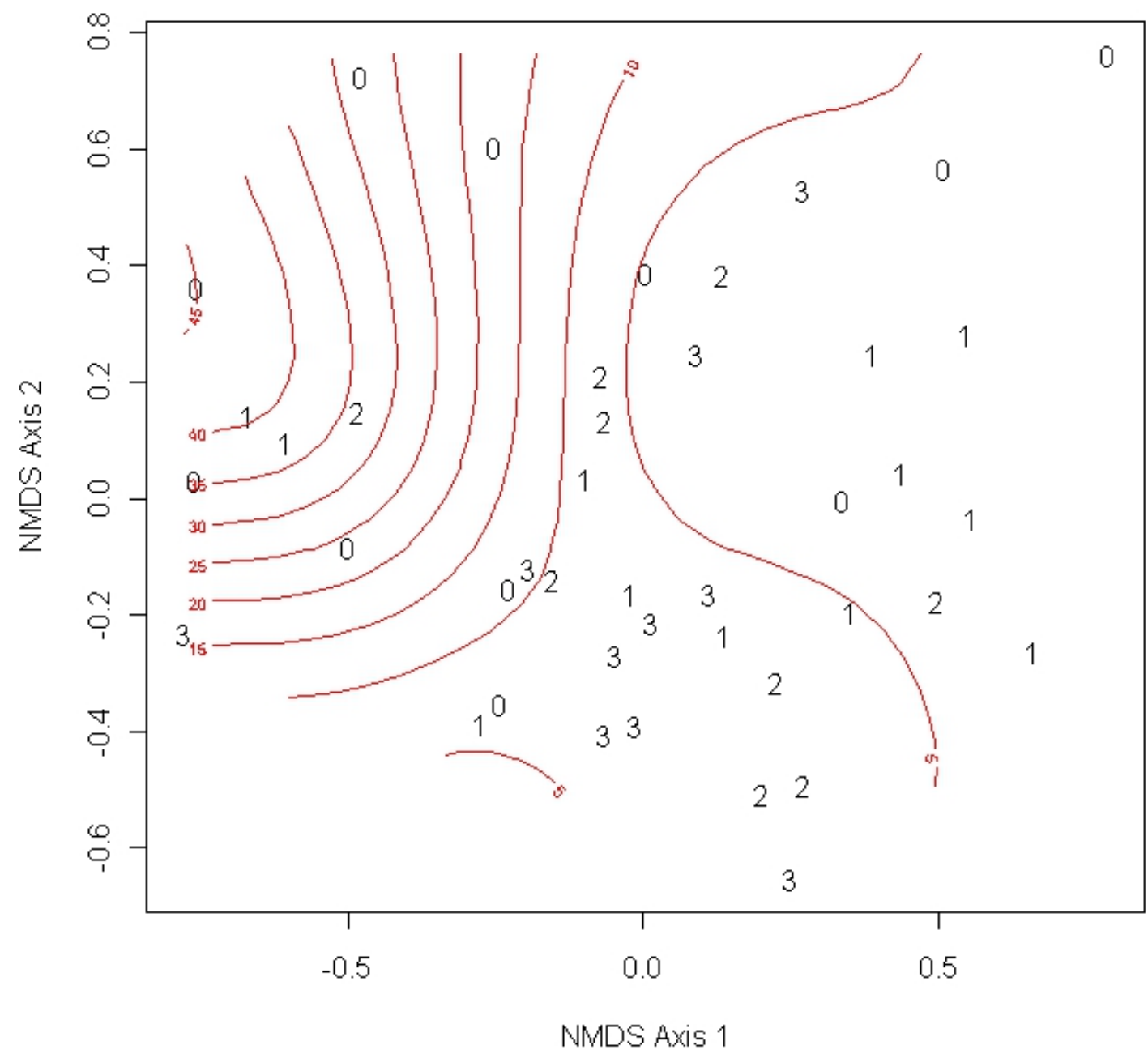

Figure 5. Crowngroups as labels: Time since last disturbance (TSLD) as surface. The majority of sites with abundant $T$. stoloniferum (17 out of 19 for Crowngroups 2 and 3) had been disturbed within the last 8 years. 


\section{Shannon's Diversity Index}



Figure 6. Crowngroups as labels: Shannon's diversity index as surface. Those sites with $T$. stoloniferum present (Crowngroups 1-3) and higher abundance sites (Crowngroups 2-3) were situated towards higher (> 2.53) values of Shannon's index along the environmental gradient. High diversity sites are in general indicative of higherquality sites for $T$. stoloniferum. 


\section{Canopy Openness (percent open sky)}

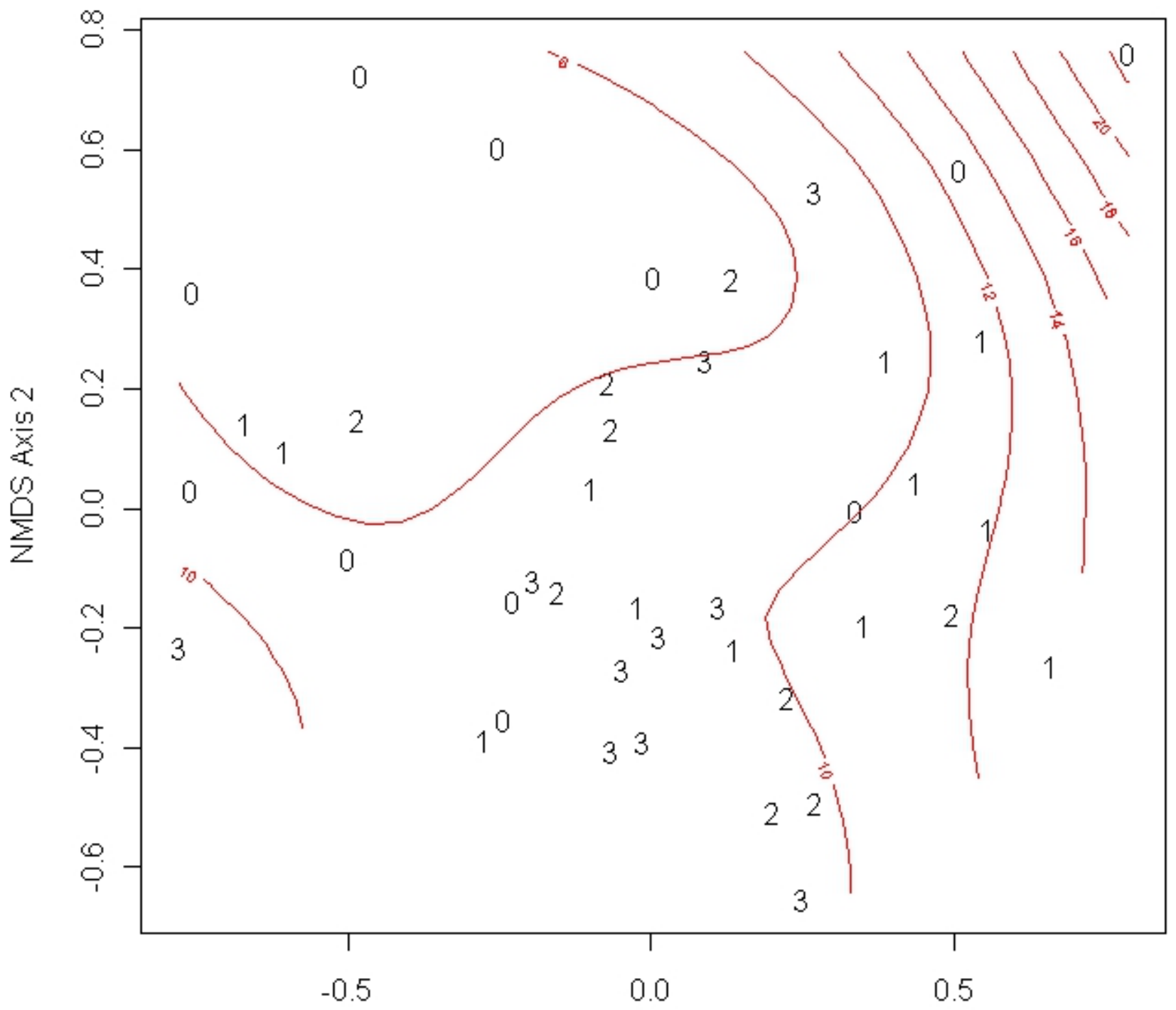

NMDS Axis 1

Figure 7. Crowngroups as labels: Canopy openness (OPEN) as surface. The majority of high abundance sites (Crowngroups 2-3) are found in situations where canopy openness was measured between $8-10 \%$ open sky. However, many control sites were also found in this canopy openness range. 
Burkhart 101

(Basal Area (sq. ft./acre)

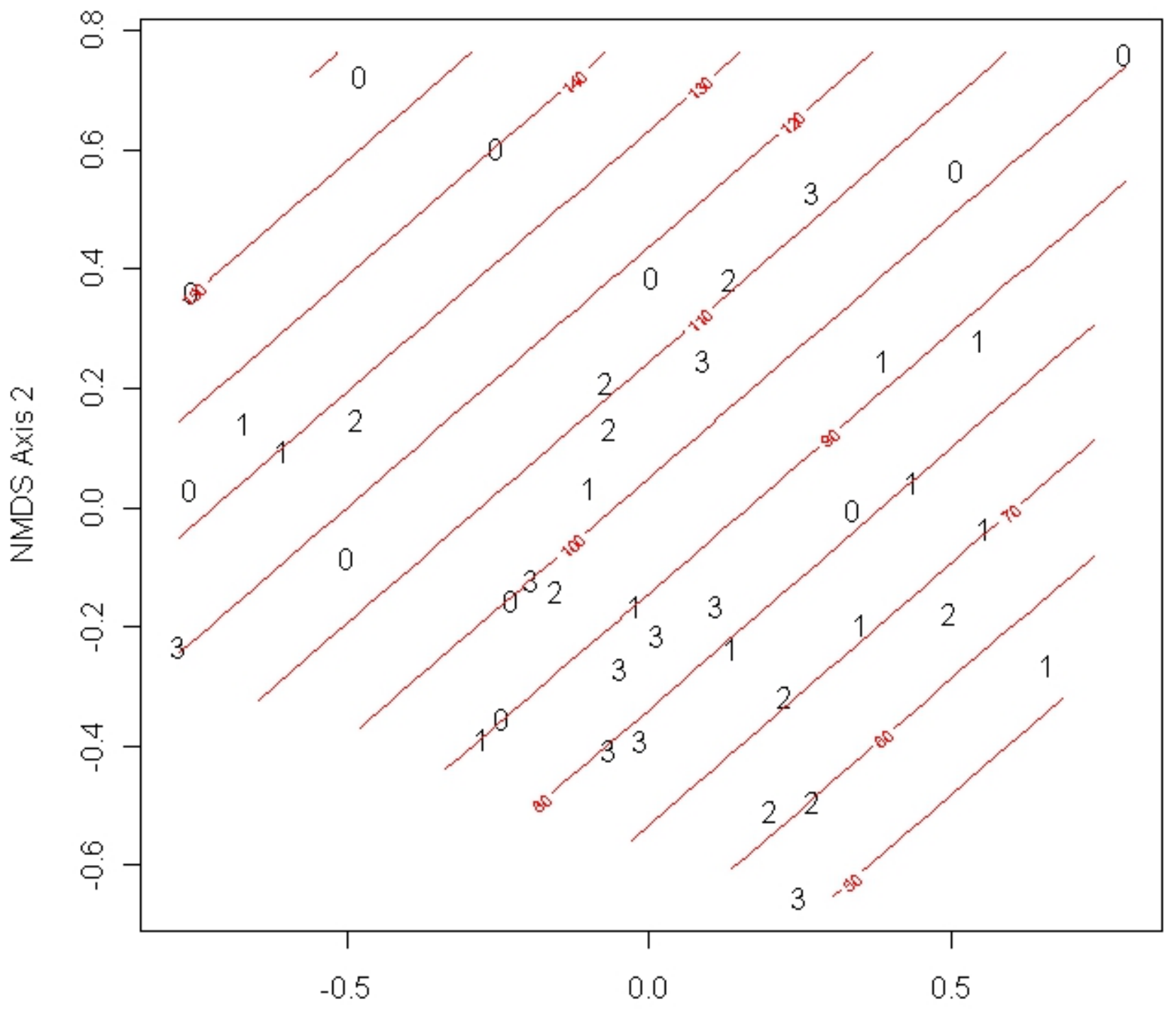

NMDS Axis 1

Figure 8. Crowngroups as labels: Basal area (BA) as surface. Those sites with $T$. stoloniferum present were found most often in forests with a basal area of trees between $60-120 \mathrm{ft}^{2} / \mathrm{ac}$. Few high-quality sites were found in forests with very high basal area of trees $\left(>120 \mathrm{ft}^{2} / \mathrm{ac}\right)$. 


\section{Shannon's Diversity Index}

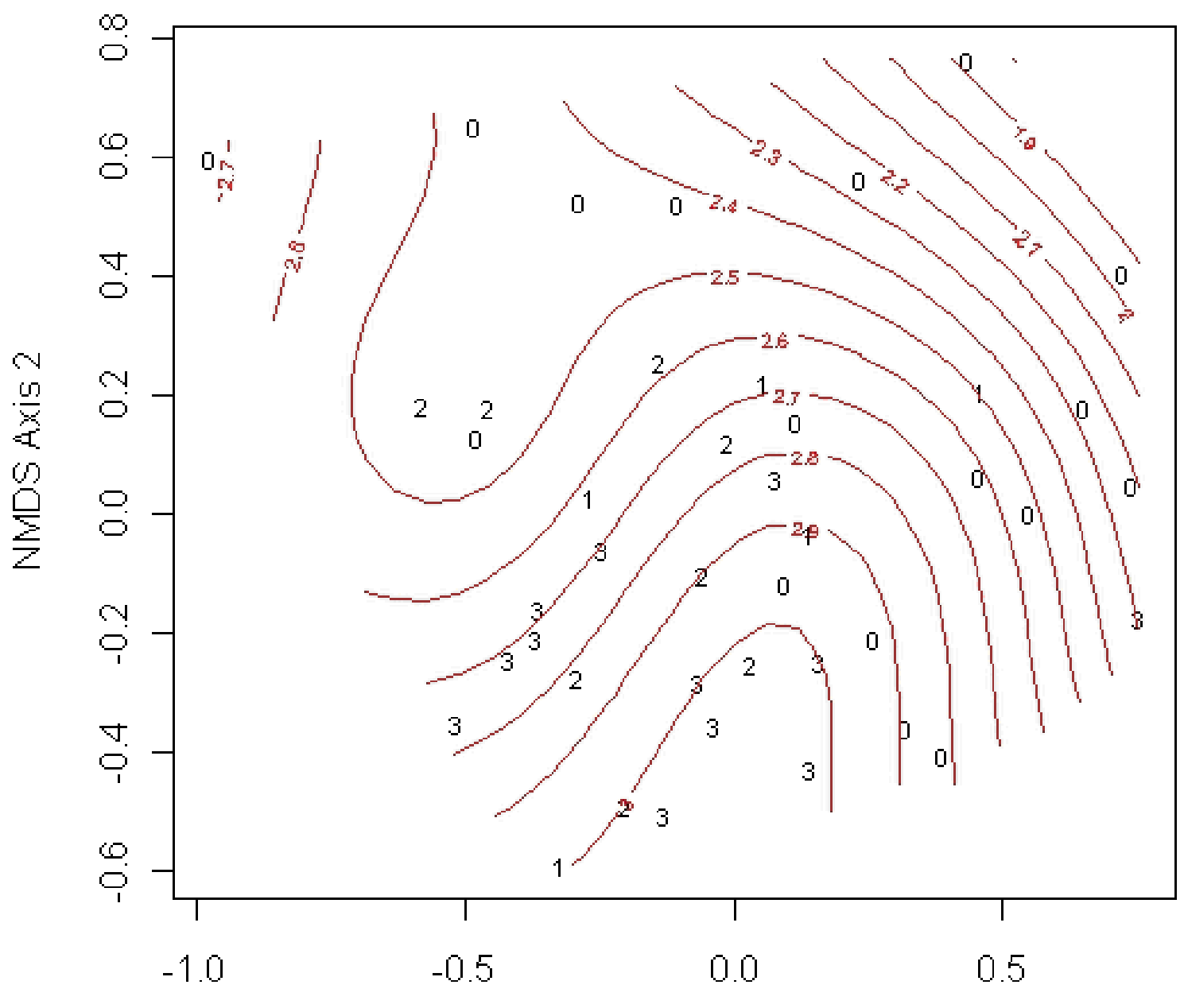

NMDS Axis 1

Figure 9. Flowergroups as labels: Shannon's diversity index (SHANNON) as surface. Those sites with high flowering index are more strongly associated with diverse sites $(>2.53)$, as measured by Shannon's index, than sites with low diversity. High diversity indicates that forest structure and disturbance regime are conducive to the development of a diverse herb layer, of which $T$. stoloniferum is a part. 
Burkhart 103

\section{Time Since Last Disturbance (years)}

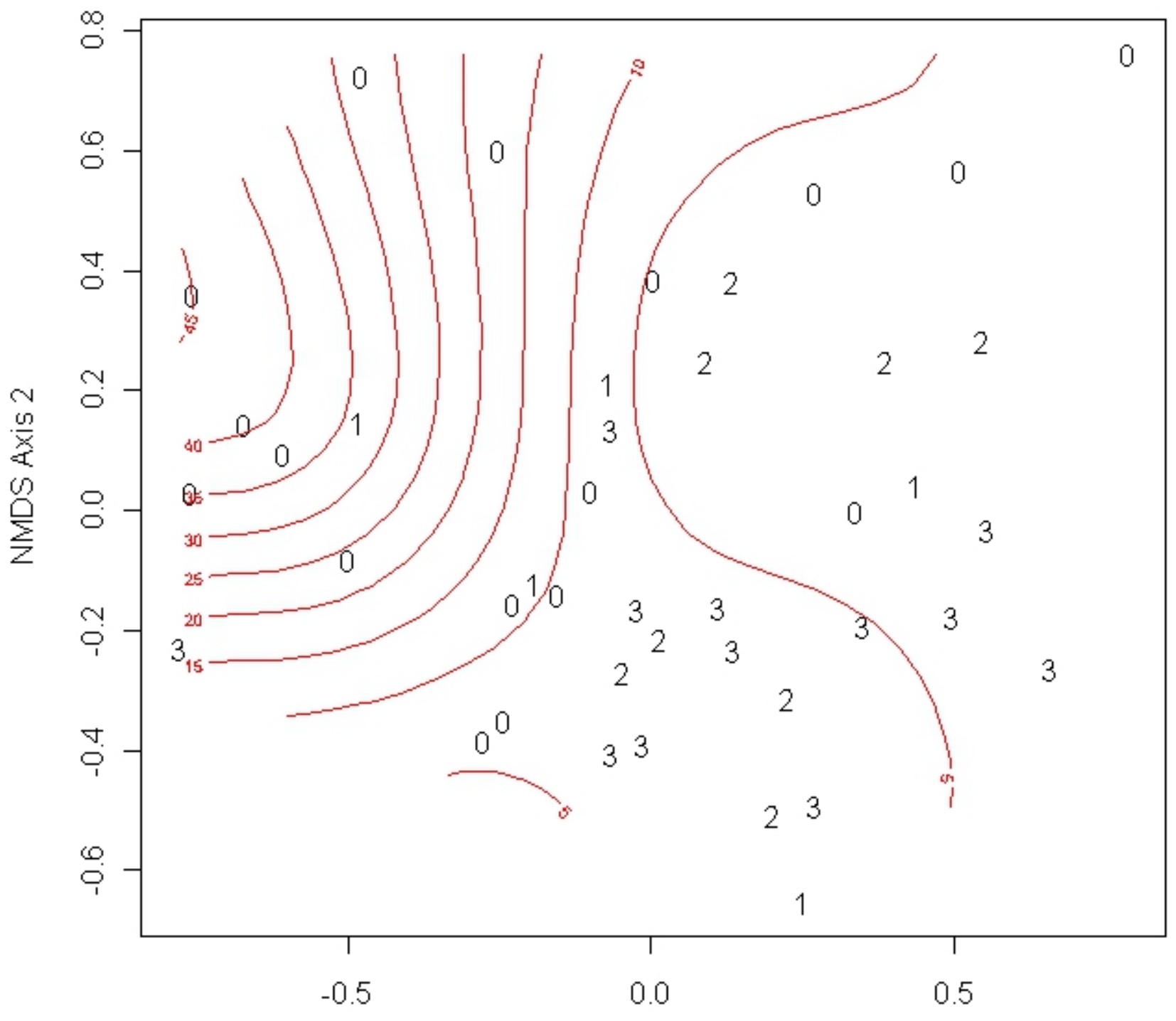

NMDS Axis 1

Figure 10. Flowergroups as labels: Time since last disturbance (TSLD) as surface. A clear pattern of sites with a TSLD $<10$ years having higher flowering index can be seen. There are also sites with low or no flowering that have been recently disturbed, but what is clear is that long intervals between disturbances are not conducive to the sexual reproduction of $T$. stoloniferum. 
Burkhart 104

\section{Basal Area (sq. ft./acre)}

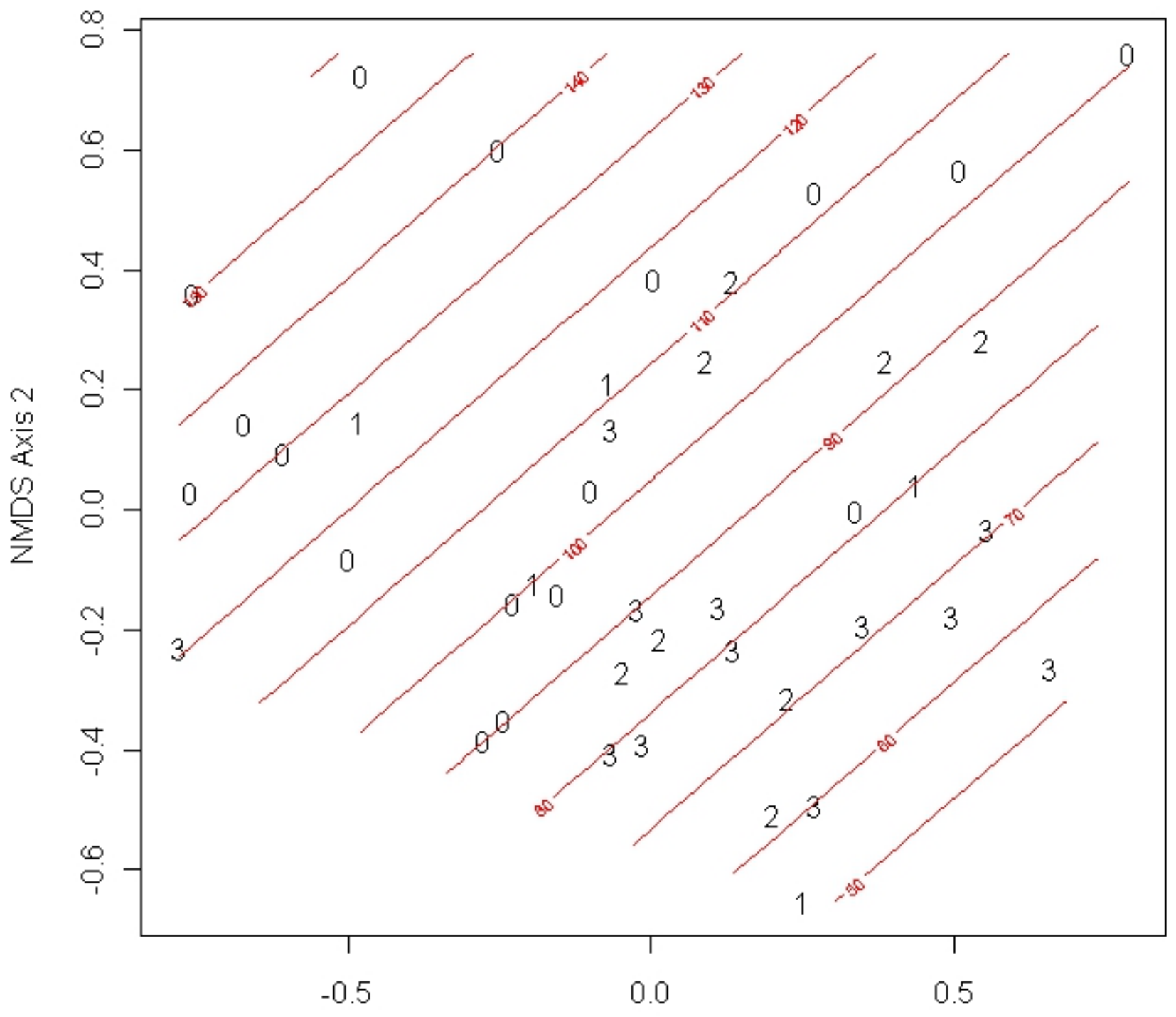

NMDS Axis 1

Figure 11. Flowergroups as labels: Basal area (BA) as surface. Those sites with high flowering indices are generally found in sites with basal areas below $110 \mathrm{ft}^{2} / \mathrm{ac}$. There is a particularly strong pattern of medium-to-high flowering index sites (Flowergroups 2 and 3) being found at sites with a basal area between 50 and $90 \mathrm{ft}^{2} /$ ac. These lower values of basal area apparently provide the light resources necessary for the sexual reproduction of $T$. stoloniferum. 


\section{Shrub Cover (sum of two transects in meters)}



NMDS Axis 1

Figure 12. Flowergroups as labels: Shrub cover (SHRUBCOV) as surface. High levels of shrub cover are associated with high levels of sexual reproduction in T. stoloniferum. High flowering index sites (Flowergroups 2 and 3) appear to be more associated with sites with greater shrub cover than sites with low shrub cover. Elevated shrub cover is most likely a response to greater light resource availability, which is also conducive to sexual reproduction by $T$. stoloniferum. 


\section{Photosynthetic Photon Flux Density ( $\mu \mathrm{mol}$ photons/m2/second)}

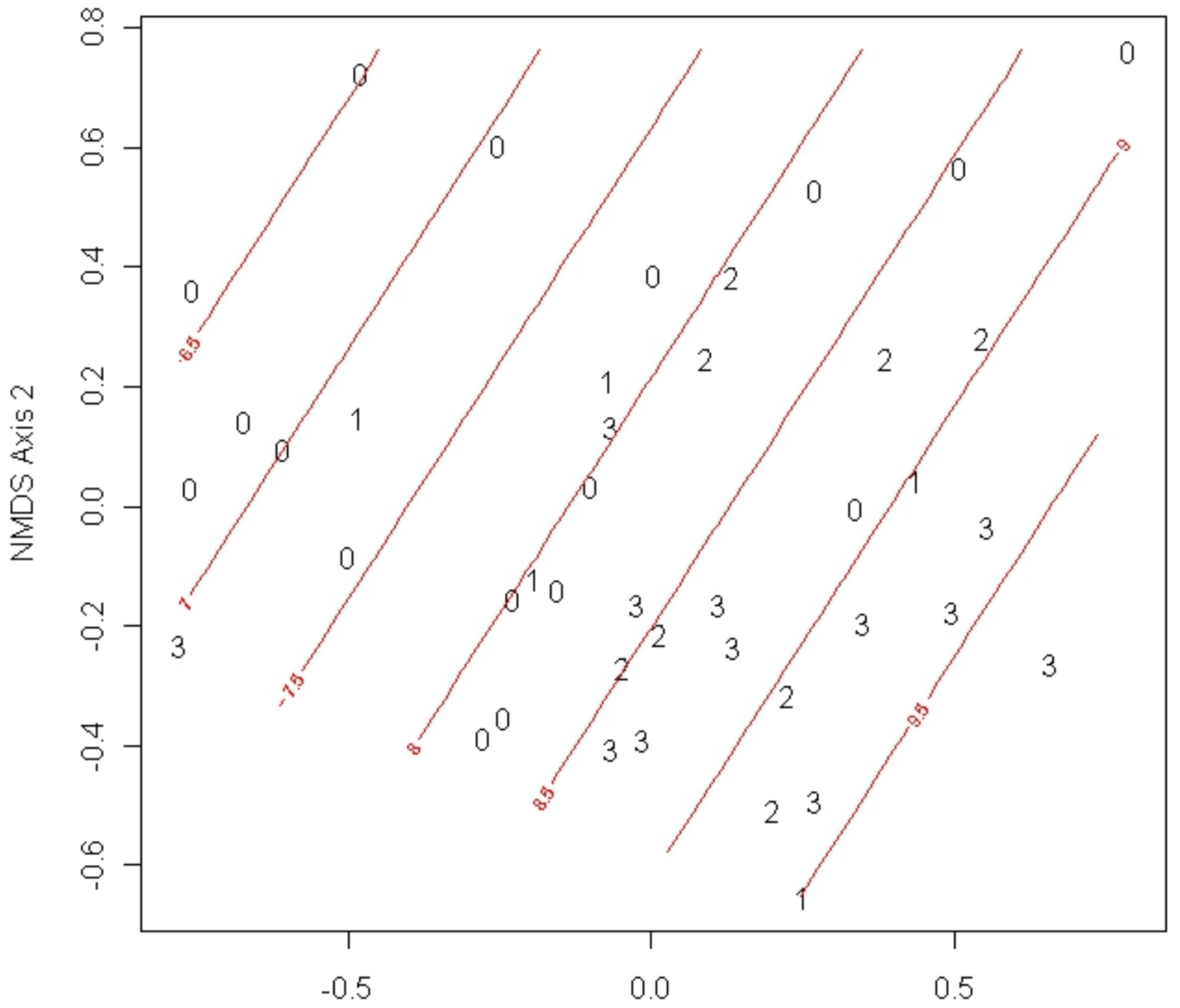

NMDS Axis 1

Figure 13. Flowergroups as labels: Photosynthetic photon flux density (PPFD) at level of forest understory. Higher light availability $\left(>8.73 \mu \mathrm{mol}\right.$ photons $\left./ \mathrm{m}^{2} / \mathrm{second}\right)$ at the level of the forest understory strongly promotes the sexual reproduction of $T$. stoloniferum. 


\section{Crowngroup with Shannon's Index}

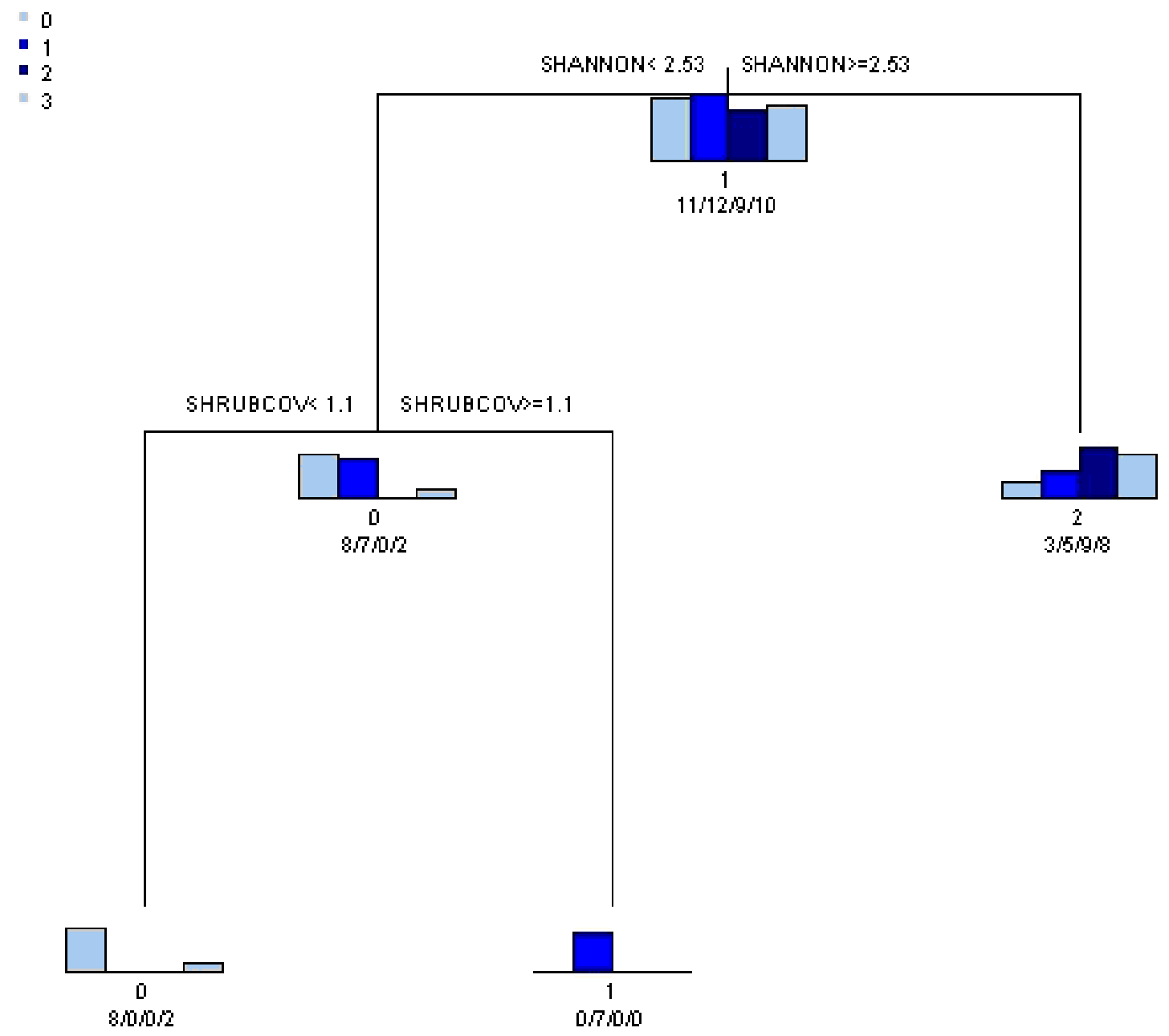

Figure 14. Classification tree analysis with Crowngroup as the response and Shannon's diversity index included in the model. (Error $=0.4, \mathrm{CV}$ error $=0.90, \mathrm{SE}=0.10$, Misclassification rates: Null $=0.71$, Model $=0.29, \mathrm{CV}=0.74$ ) 


\section{Crowngroup (Shannon's \\ Index Excluded)}

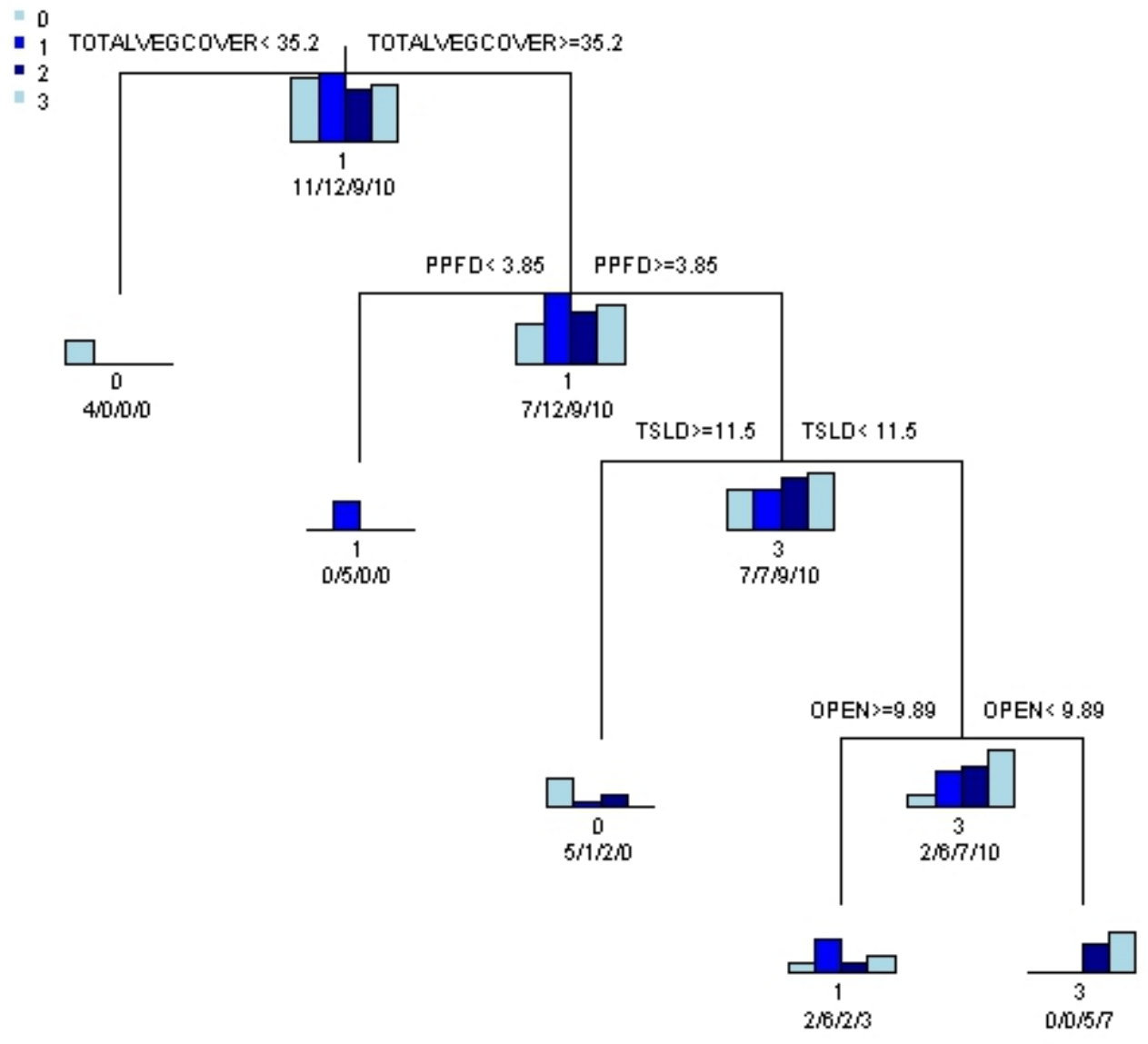

Figure 15. Classification tree analysis with Crowngroup as the response and Shannon's diversity index excluded from the model. (Error $=0.30, \mathrm{CV}$ error $=1.03, \mathrm{SE}=0.095$, Misclassification rates: Null $=0.714$, Model $=0.21, \mathrm{CV}=0.738$ ) 


\section{Flowergroup}

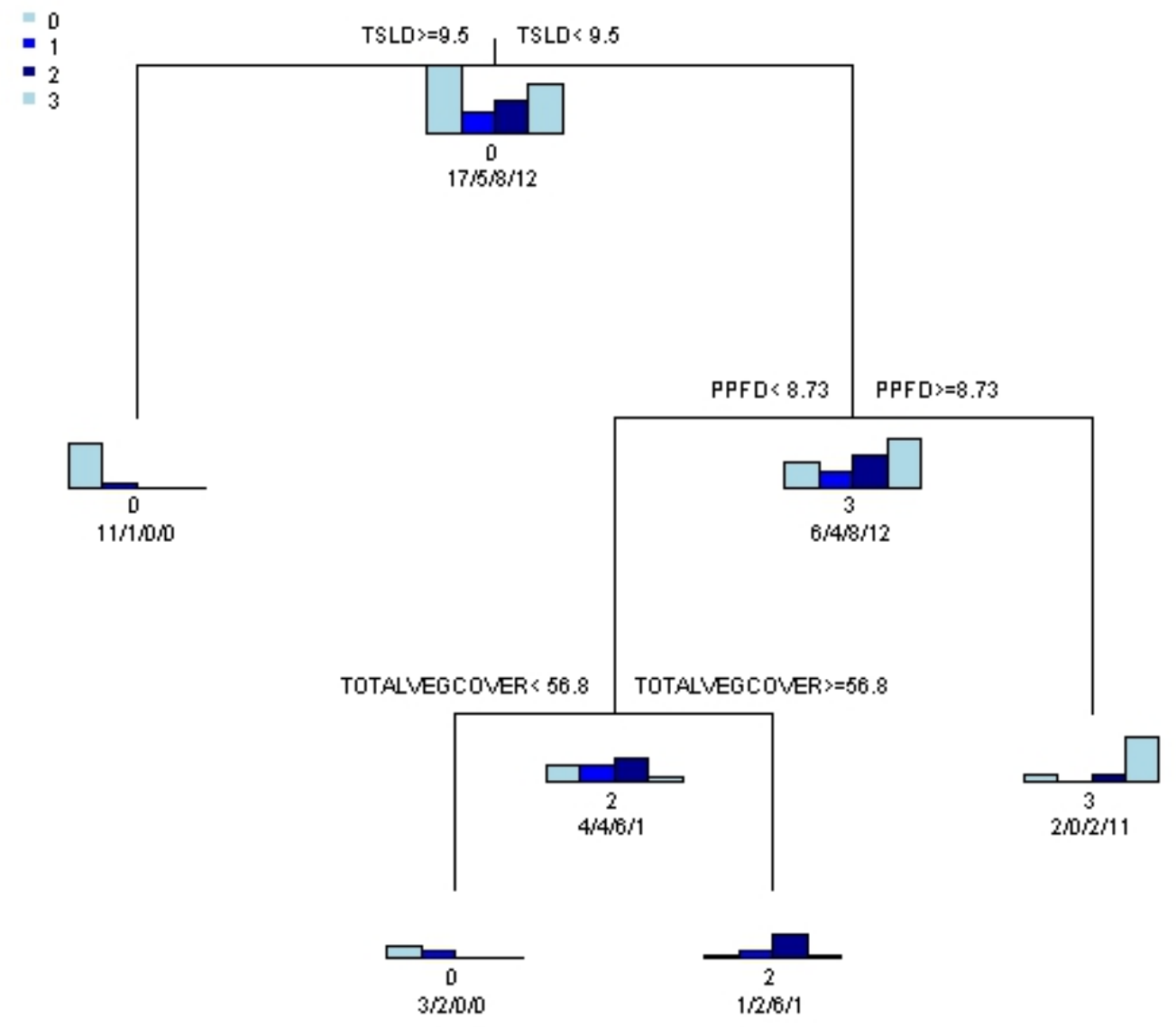

Figure 16. Classification tree analysis with Flowergroup as response variable. (Error $=0.76, \mathrm{CV}$ error $=0.92, \mathrm{SE}=0.13$, Misclassification rates: Null $=0.60$, Model $=0.45$, $\mathrm{CV}=0.54)$ 


\section{Appendix}

\section{R Code Appendix}

\section{MANOVA}

$>$ RBC.matrix $=$ read.table(file='envmatrixparsimony.csv', header=TRUE, sep=',')

$>$ habitat.RBC.transform=data.frame(cbind(coAspect, logTSLD, BA, log1pSAPTALLY, $\log 1$ pSHRUBCOV, TOTALVEGCOVER, OPEN, PPFD, SHANNON)) $>$ manova.Crowngroup $=$ manova(as.matrix(habitat.RBC.transform) as. factor(Crowngroup)) $>$ summary(manova.Crowngroup)

$>$ manova.Flowergroup $=$ manova(as.matrix(habitat.RBC.transform) $\sim$ as. factor(Flowergroup) summary(manova.Flowergroup)

\section{NMDS Analyses}

$>$ library(vegan)

$>$ RBC.matrix $=$ read.table $($ file $=$ 'envmatrixparsimonywithvegrareeliminated.csv', header=TRUE, sep=',')

$>$ NMDS3.RBC $=$ metaMDS(RBC.matrix[,17:83], $\mathrm{k}=3)$

$>$ envfit3.RBC=envfit(NMDS3.RBC, RBC.matrix[,5:16], permu=1000)

$>\operatorname{attach}(\mathrm{RBC} . \mathrm{matrix})$

$>$ envfit3.RBC.hs.Crowngroup=envfit(NMDS3.RBC $\sim$ Flowergroup+TSLD+BA+SAPTALLY

+ SHRUBCOV+TOTALVEGCOVER+OPEN+PPFD+SHANNON, permu=1000)

$>$ envfit3.RBC.hs.Flowergroup $=$ envfit(NMDS3.RBC $\sim$ Crowngroup+TSLD+BA+SAPTALLY

+ SHRUBCOV+TOTALVEGCOVER+OPEN+PPFD+SHANNON, permu=1000)

envfit3.RBC=envfit(NMDS3.RBC, NMDS.RBC[,75:94], permu=1000)

\section{\#FOR DISPLAY OF SURFACES}

$>$ plot(NMDS3.RBC\$points, type="n", xlab="NMDS Axis 1", ylab="NMDS Axis 2", main="Vector overlay with Crowngroups as labels")

$>$ plot(envfit3.RBC.hs.Crowngroup, font=0.7, col="blue")

$>$ text(NMDS3.RBC\$points, labels=as.character(RBC.matrix \$Crowngroup), cex=0.7)

$>\#$ This process is iterated for every variable one wishes to display as a surface. The main $>\#$ title, the label of the points, and the variable to be projected as a surface will change.

\section{\#FOR DISPLAY OF VECTORS}

>plot(NMDS3.RBC\$points, type="n", xlab="NMDS Axis 1", ylab="NMDS Axis 2", main="Vector overlay with Flowergroups as labels")

$>$ plot(envfit3.RBC.hs.Flowergroup, font $=0.7$, col="blue")

$>$ text(NMDS3.RBC\$points, labels=as.character(RBC.matrix\$Flowergroup), cex $=0.7$ ) 


\section{Classification tree analyses}

library(mvpart)

RBCcart=read.table(file="CARTmatrixRBC.csv", header=TRUE, sep=",")

Crowngroup.tree $=$ mvpart $($ Crowngroup $\sim$ TSLD + BA + SAPTALLY+SHRUBCOV+TOTALVE GCOVER+OPEN+PPFD+SHANNON, data=RBCcart)

Flowergroup.tree $=$ mvpart(FI TSLD+BA+SAPTALLY+SHRUBCOV+TOTALVEGCOVER + OPEN+PPFD+SHANNON, data=RBCcart)

\# can use different cross-validation methods: $x v=$ 'none', $x v=' p$ ', $x v=" 1 s e ", x v=" m i n "$. \#These different cross-validation methods allow for the construction of trees of varying \#lengths. 
Appendix 1. Site Identifiers. Patch ID is the name given by personnel of the Fernow Experimental Forest to a monitored T. stoloniferum occurrence. Occurrences are censused biennially and rooted crowns are recorded. Crowngroups 1-3 represent extant sites (Crowngroup 1=1-29 rooted crowns, Crowngroup 2=30-99, Crowngroup $3=\geq 100$ ). Crowngroup 0 consists of sites where $T$. stoloniferum was once found but has become extirpated or sites chosen as control sites because they met basic environmental requirements of $T$. stoloniferum, such as being underlain by calcareous soils, but differed in disturbance regime from sites which possessed $T$. stoloniferum. Crowns is the number of rooted crowns recorded in the last census. INFL is the number of inflorescences recorded in May/June 2009. FI is flowering index, which is INFL/Crowns and gives a relative measure of reproductive success. Flowergroups are based upon FI (Flowergroup 0=0, Flowergroup $1=0.01-0.05$, Flowergroup $2=0.06-0.19$, Flowergroup $3 \geq 0.20$ ). TSLD is the time in years since the last logging-related disturbance. BA is the basal area measured in square feet/acre as measured by a 10 basal area factor prism spun around the center of the $100 \mathrm{~m}^{2}$ habitat assessment plot. SAPTALLY is a tally of saplings within the $100 \mathrm{~m}^{2}$ habitat assessment plot. SHRUBCOV is the total length in meters that shrubs intersected two diagonal $14.1 \mathrm{~m}$ transects stretched from the corners of the habitat assessment plot. TOTALVEG is the mean percent cover of all vascular plants less than $1 \mathrm{~m}$ tall recorded in $51 \mathrm{~m}^{2}$ circular plots located in the habitat assessment plot. OPEN and PPFD were assessed by means of hemispherical photography and photograph analysis by Winscanopy 2006a ((Regent Instruments INC. 2006). OPEN is the percent open sky and PPFD is the photosynthetic photon flux density, a measure of photosynthetically active radiation which is measured in units of $\mu \mathrm{mol}$ photons $/ \mathrm{m}^{2} / \mathrm{second}$.

\begin{tabular}{|c|c|c|c|c|c|c|c|c|c|c|}
\hline Patch ID & $\begin{array}{l}\text { Crown } \\
\text { group }\end{array}$ & $\begin{array}{r}\text { Flower } \\
\text { group }\end{array}$ & Crowns & INFL & FI & TSLD & $\mathrm{BA}$ & TOTALVEG & OPEN & PPFD \\
\hline $16-1$ & 0 & 0 & 0 & 0 & 0 & 19 & 90 & 33.66 & 6.3 & 3.82 \\
\hline $17 \mathrm{~A}$ & 0 & 0 & 0 & 0 & 0 & 2 & 120 & 18.04 & 12.62 & 11.37 \\
\hline $7 \mathrm{C}$ & 0 & 0 & 0 & 0 & 0 & 7 & 50 & 48.6 & 11.9 & 3.92 \\
\hline BSG5 & 0 & 0 & 0 & 0 & 0 & 56 & 110 & 95 & 9.4 & 8.84 \\
\hline $\begin{array}{r}\text { Control 14- } \\
2\end{array}$ & 0 & 0 & 0 & 0 & 0 & 56 & 140 & 53.6 & 9.02 & 7.81 \\
\hline $\begin{array}{r}\text { Control 18- } \\
1\end{array}$ & 0 & 0 & 0 & 0 & 0 & 4 & 100 & 50.2 & 24.91 & 11.92 \\
\hline $\begin{array}{r}\text { Control 18- } \\
3\end{array}$ & 0 & 0 & 0 & 0 & 0 & 4 & 100 & 28 & 1.84 & 0.89 \\
\hline $\begin{array}{r}\text { Control 43- } \\
2\end{array}$ & 0 & 0 & 0 & 0 & 0 & 19 & 160 & 57.5 & 6.95 & 5.69 \\
\hline $\begin{array}{r}\text { Control 43- } \\
3\end{array}$ & 0 & 0 & 0 & 0 & 0 & 19 & 160 & 43.46 & 6.64 & 7 \\
\hline $\begin{array}{r}\text { Control 70- } \\
1\end{array}$ & 0 & 0 & 0 & 0 & 0 & 15 & 70 & 61.4 & 3.9 & 7 \\
\hline $\begin{array}{r}\text { Control 70- } \\
2\end{array}$ & 0 & 0 & 0 & 0 & 0 & 15 & 130 & 34.1 & 6.92 & 14 \\
\hline $72-7$ & 1 & 0 & 30 & 0 & 0 & 11 & 100 & 36.3 & 5.04 & 2.64 \\
\hline
\end{tabular}


Burkhart 113

\begin{tabular}{|c|c|c|c|c|c|c|c|c|c|c|}
\hline $9-41$ & 1 & 0 & 10 & 0 & 0 & 8 & 70 & 54.8 & 9.34 & 1.46 \\
\hline BSG2-A & 1 & 0 & 2 & 0 & 0 & 33 & 120 & 47.6 & 7.16 & 6.22 \\
\hline BSG2-B & 1 & 0 & 15 & 0 & 0 & 33 & 110 & 59.2 & 5.73 & 3.72 \\
\hline 20A-m & 1 & 1 & 5 & 1 & 0.2 & 2 & 60 & 79.74 & 12.84 & 5.07 \\
\hline 20B-STA1 & 1 & 2 & 63 & 6 & 0.1 & 3 & 90 & 58.4 & 8.74 & 2.2 \\
\hline $13 \mathrm{~A}-1$ & 1 & 3 & 16 & 27 & 1.69 & 6 & 90 & 52.1 & 12.73 & 14.79 \\
\hline $20 \mathrm{~A}-\mathrm{i}$ & 1 & 3 & 27 & 7 & 0.26 & 2 & 70 & 78.54 & 14.07 & 18.76 \\
\hline 20B-STA3 & 1 & 3 & 21 & 5 & 0.24 & 3 & 110 & 71.6 & 9.63 & 3.83 \\
\hline $9-28 \mathrm{~A}$ & 1 & 3 & 20 & 26 & 1.3 & 8 & 40 & 59.9 & 12.02 & 13.69 \\
\hline $9-45$ & 1 & 3 & 97 & 28 & 0.29 & 8 & 50 & 87.4 & 13.7 & 11.46 \\
\hline $16-6$ & 2 & 0 & 38 & 0 & 0 & 19 & 140 & 41.7 & 12.46 & 8.18 \\
\hline BSG2-C & 2 & 1 & 33 & 1 & 0.03 & 33 & 150 & 69.7 & 7.12 & 9.46 \\
\hline BSG7f & 2 & 1 & 62 & 2 & 0.03 & 1 & 140 & 50 & 5.39 & 6.45 \\
\hline $9-13$ & 2 & 2 & 50 & 4 & 0.08 & 7 & 70 & 57.9 & 9.84 & 9.2 \\
\hline $9-33$ & 2 & 2 & 60 & 8 & 0.13 & 7 & 70 & 64.5 & 7.26 & 6.36 \\
\hline BSG7G & 2 & 2 & 76 & 5 & 0.07 & 1 & 60 & 68.2 & 9.94 & 7.72 \\
\hline WS5 & 2 & 2 & 72 & 7 & 0.1 & 2 & 100 & 104.6 & 5.49 & 4 \\
\hline $20 \mathrm{~A}-\mathrm{b}$ & 2 & 3 & 36 & 23 & 0.64 & 2 & 70 & 77.8 & 17.32 & 9.96 \\
\hline $20 \mathrm{~A}-\mathrm{e}$ & 2 & 3 & 41 & 15 & 0.37 & 2 & 50 & 56.3 & 12.84 & 15.4 \\
\hline $9-36$ & 2 & 3 & 67 & 43 & 0.64 & 7 & 60 & 61.2 & 8.04 & 8.74 \\
\hline BSG8 & 3 & 0 & 227 & 0 & 0 & 1 & 110 & 70.1 & 7.32 & 6.62 \\
\hline $9-14$ & 3 & 1 & 309 & 4 & 0.01 & 8 & 20 & 55.2 & 7.94 & 7.07 \\
\hline $9-52 / 53$ & 3 & 1 & 125 & 2 & 0.02 & 7 & 110 & 67.8 & 5.35 & 3.87 \\
\hline $20 A-d$ & 3 & 2 & 131 & 13 & 0.1 & 2 & 90 & 50.4 & 14.68 & 11.86 \\
\hline $9-19$ & 3 & 2 & 565 & 108 & 0.19 & 7 & 60 & 76.4 & 9.83 & 8.15 \\
\hline $9-25$ & 3 & 2 & 342 & 21 & 0.06 & 7 & 110 & 74.4 & 9.25 & 8.72 \\
\hline $9-37$ & 3 & 3 & 278 & 65 & 0.23 & 7 & 90 & 54.7 & 8.18 & 16.77 \\
\hline $9-46$ & 3 & 3 & 461 & 140 & 0.3 & 7 & 100 & 91.2 & 13.39 & 10.32 \\
\hline BSG6 & 3 & 3 & 178 & 56 & 0.31 & 2 & 210 & 50.3 & 13.61 & 12.54 \\
\hline BSG7 & 3 & 3 & 234 & 48 & 0.21 & 1 & 140 & 72.4 & 5.91 & 9.38 \\
\hline
\end{tabular}


Appendix 2. Photographs and descriptions of study sites.

In this appendix, I provide a visual representation of eleven T. stoloniferum sites of varying levels of success and four control sites without $T$. stoloniferum at the Fernow Experimental Forest. "A picture is worth a thousand words," so I hope these photographs with accompanying description can inform managers and supplement the quantitative and qualitative information I have presented in this chapter of my thesis.

I have been flexible with my use and reporting of means and standard deviations. I report and omit these when I think it will be helpful to the reader. All of the values for an individual site and means for Crowngroups and Flowergroups can be found in Appendix 1 and Tables 1 and 2 , respectively.

I begin with really good sites: those sites with high abundance of rooted crowns and high flowering index. These sites were categorized in Crowngroup 3-those sites with $\geq 100$ rooted crowns- and Flowergroup 3- those sites with a flowering index (\# of inflorescences/\# of rooted crowns) greater than or equal to 0.20 .

\section{1) Patch 9-46: Crowngroup 3, Flowergroup 3}

Patch 9-46 was the second most abundant site with 461 rooted crowns and had a high reproductive success of 140 inflorescences for a flowering index of 0.30 .

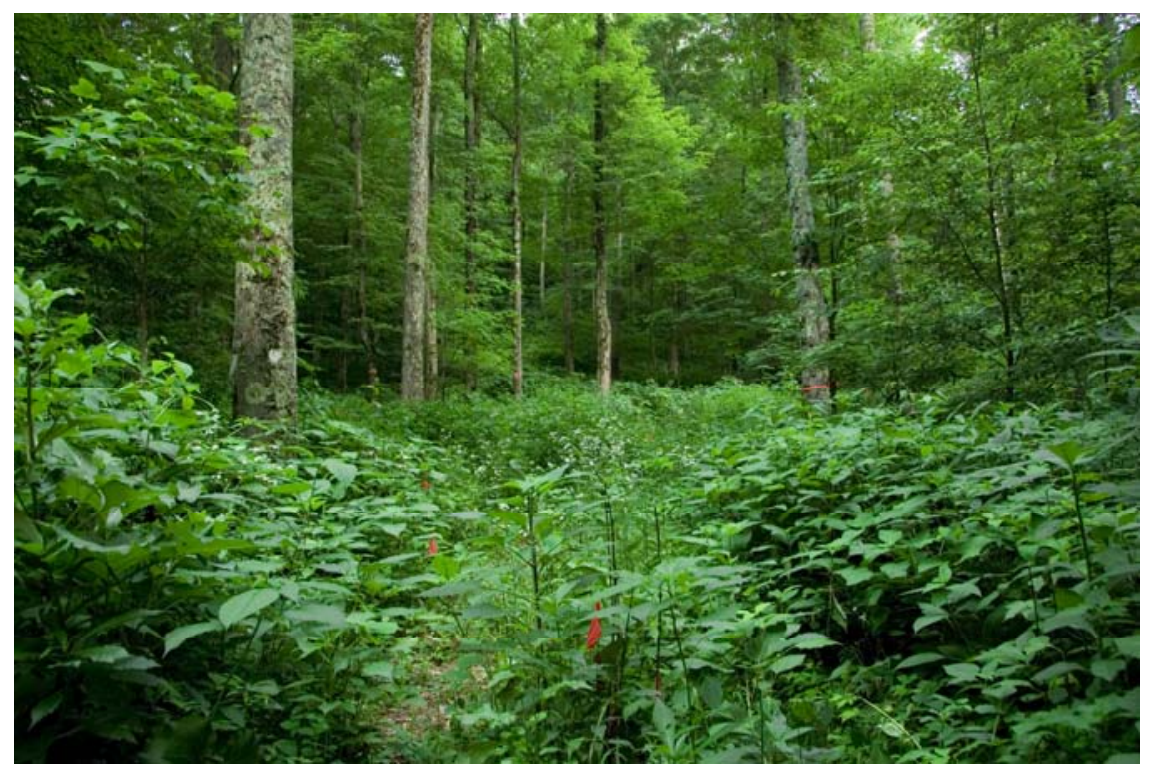

Vegetation looking uphill 
The time since last disturbance for 9-46 was 7 years, basal area was $100 \mathrm{ft}^{2} / \mathrm{ac}$, and the sapling tally was 11 saplings per $100 \mathrm{~m}^{2}$. Total herb layer cover was $54.7 \%$, which was lower than the mean for Crowngroup 3 (66.29\% SD) and Flowergroup 3 (67.79\%). Shrub cover was relatively low at 2.8 meters intersecting with transects $(2.7 \mathrm{~m}$ of cover was by various Rubus species).

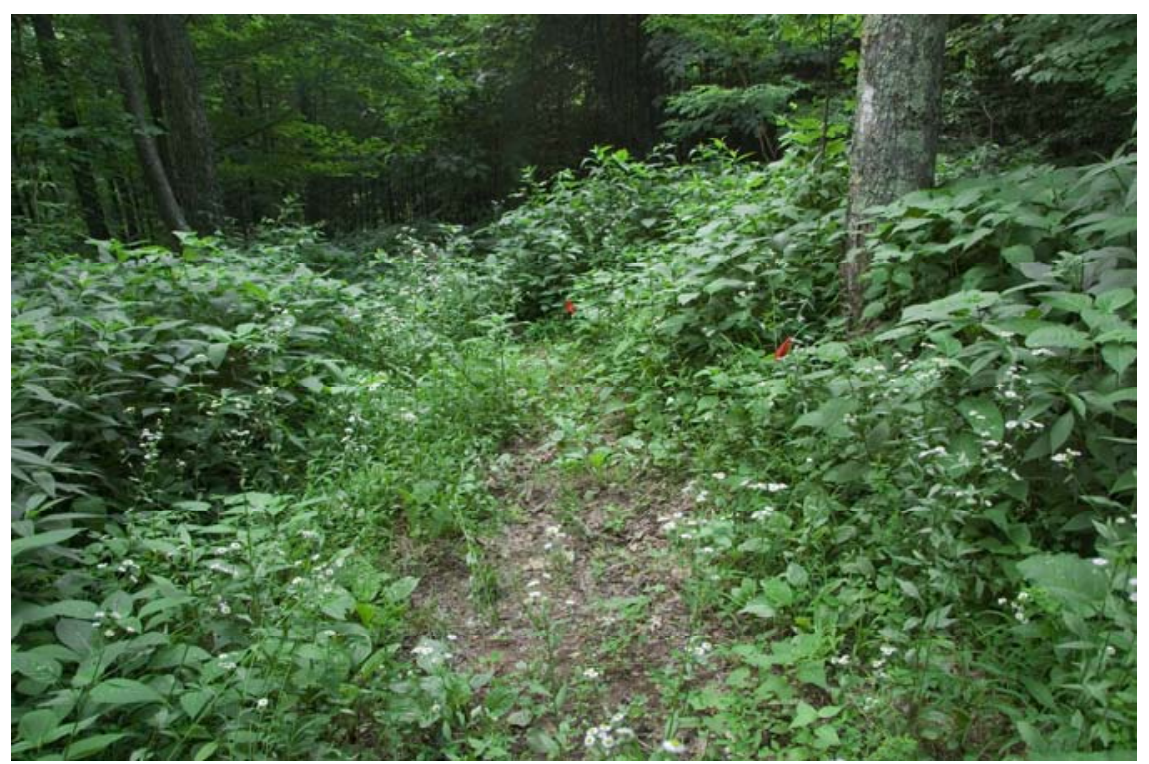

\section{Vegetation looking downhill}

The herbaceous cover at 9-46 was dominated by tall coarse members of the family Asteraceae, including Eupatorium rugosum, Helianthus decapetalus, and Verbesina alternifolia.

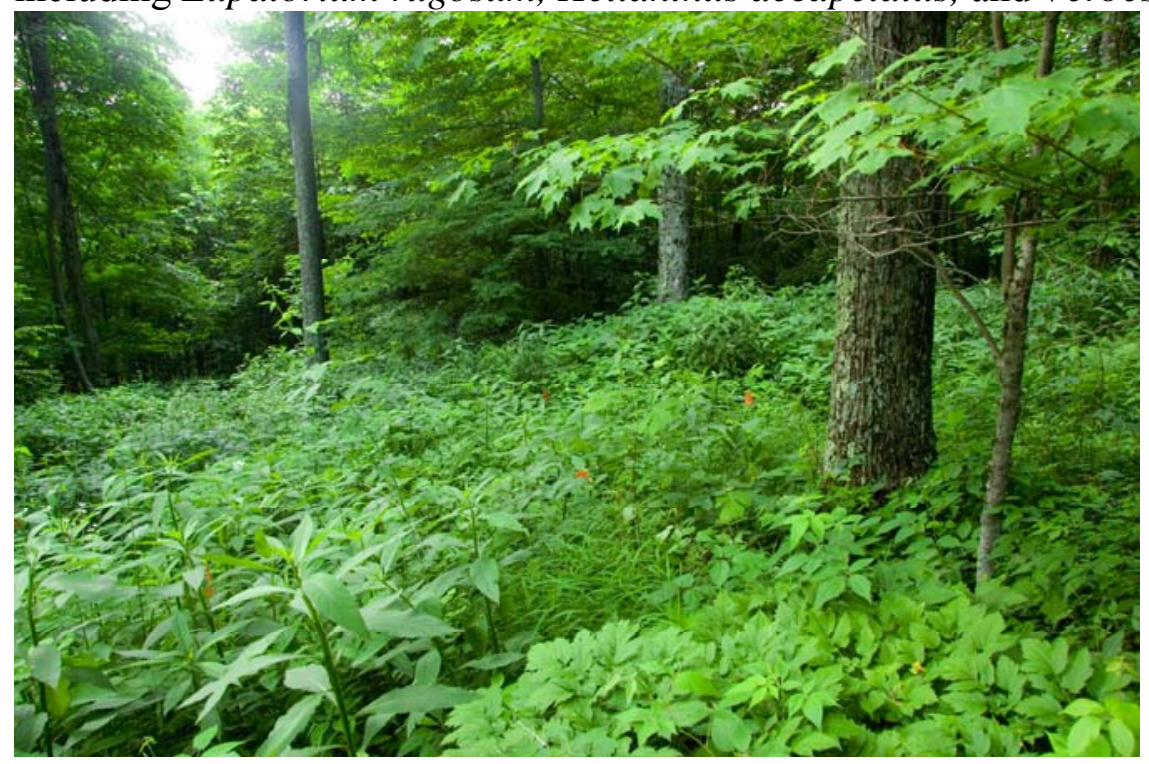

Tall, coarse herbs present in abundance 
This photograph demonstrates the open, park-like structure of the forest surrounding this optimal site for T. stoloniferum.

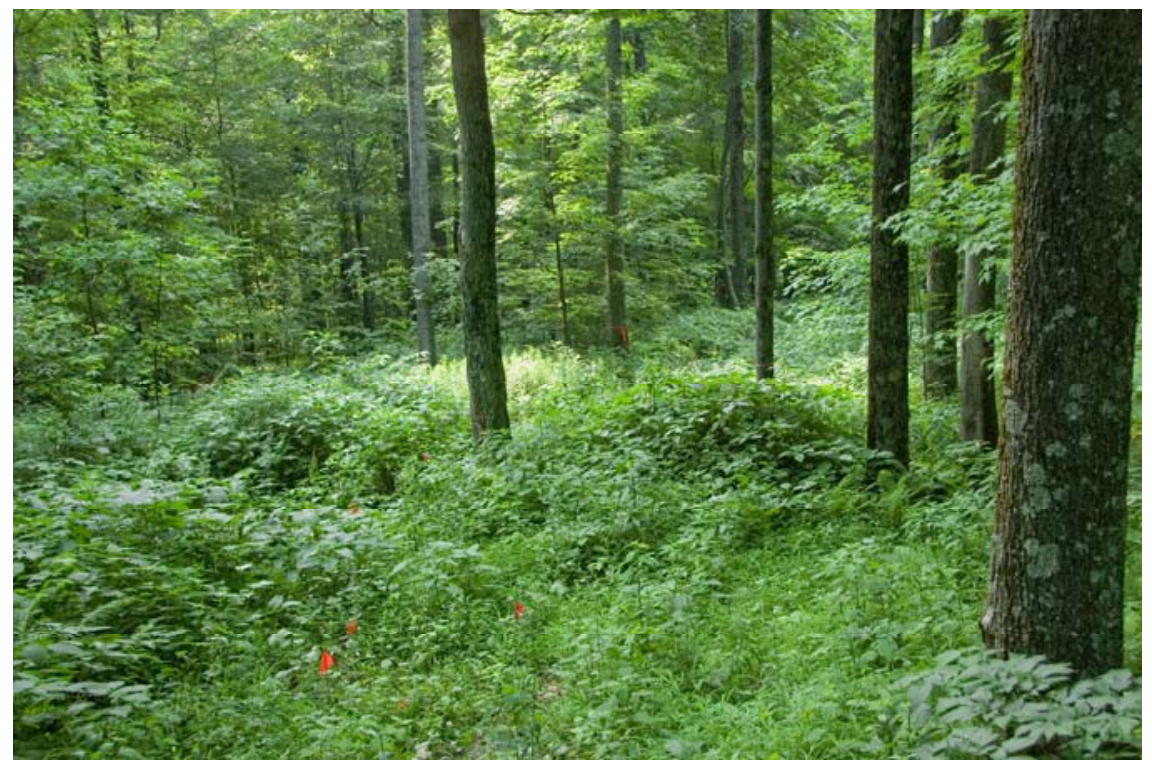

\section{Rich forested opening created by timber harvesting}

Patch 9-46 appeared to have an optimal light level and canopy structure, which was measured at $10.32 \mu \mathrm{mol}$ photons $/ \mathrm{m}^{2} /$ second and $13.39 \%$ open sky. The light level was above that of other members of Crowngroup 3 (mean $9.53 \mu \mathrm{mol}$ photons $/ \mathrm{m}^{2} / \mathrm{second}$ (SD 3.60)) and below that of other members of Flowergroup 3 (12.14 $\mu \mathrm{mol}$ photons $/ \mathrm{m}^{2} / \mathrm{second}$ (SD 4.07)).



Canopy structure at a succesful $T$. stoloniferum site. 
This photograph exemplifies a serious threat to the management of forests at the Fernow Experimental Forest. Microstegium vimineum, Japanese stilt grass, is an invasive plant and is often dispersed by logging equipment. Trifolium stoloniferum is also carried by logging equipment, so efforts to control the spread of $M$. vimineum, such as washing logging equipment, will most likely inhibit the incidental spread of $T$. stoloniferum propagules during the course of harvesting activities. Balancing beneficial management of Trifolium stoloniferum and risk mitigation is an ongoing challenge, not only at the Fernow Experimental Forest but throughout the range of this species.

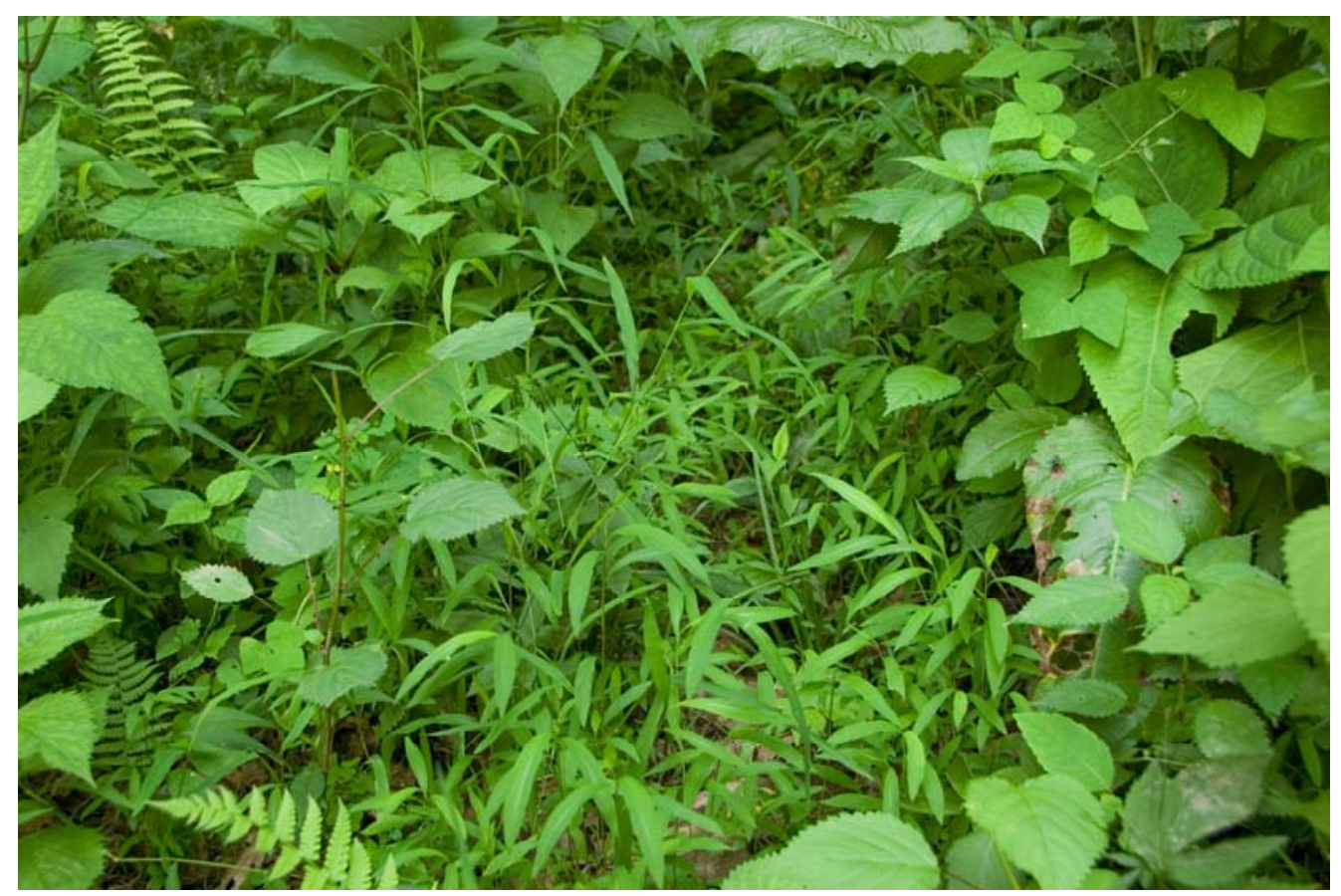

Invasive grass Microstegium vimineum present at $T$. stoloniferum site. 


\section{2) Patch BSG6: Crowngroup 3, Flowergroup 3}

Big Springs Gap 6 is located along the main road, FS 701, at the Fernow Experimental Forest. It is periodically squished by people driving over it or parking their cars on top of it. The calcareous qualities of the soil are enriched by the crushed gravel used to create the road. It is obviously highly disturbed, yet the road appears to prevent canopy closure and maintain light conditions conducive to T. stoloniferum success. BSG6 had the highest recorded basal area of any sites I measured at the Fernow Experimental Forest- $210 \mathrm{ft}^{2} / \mathrm{ac}$.

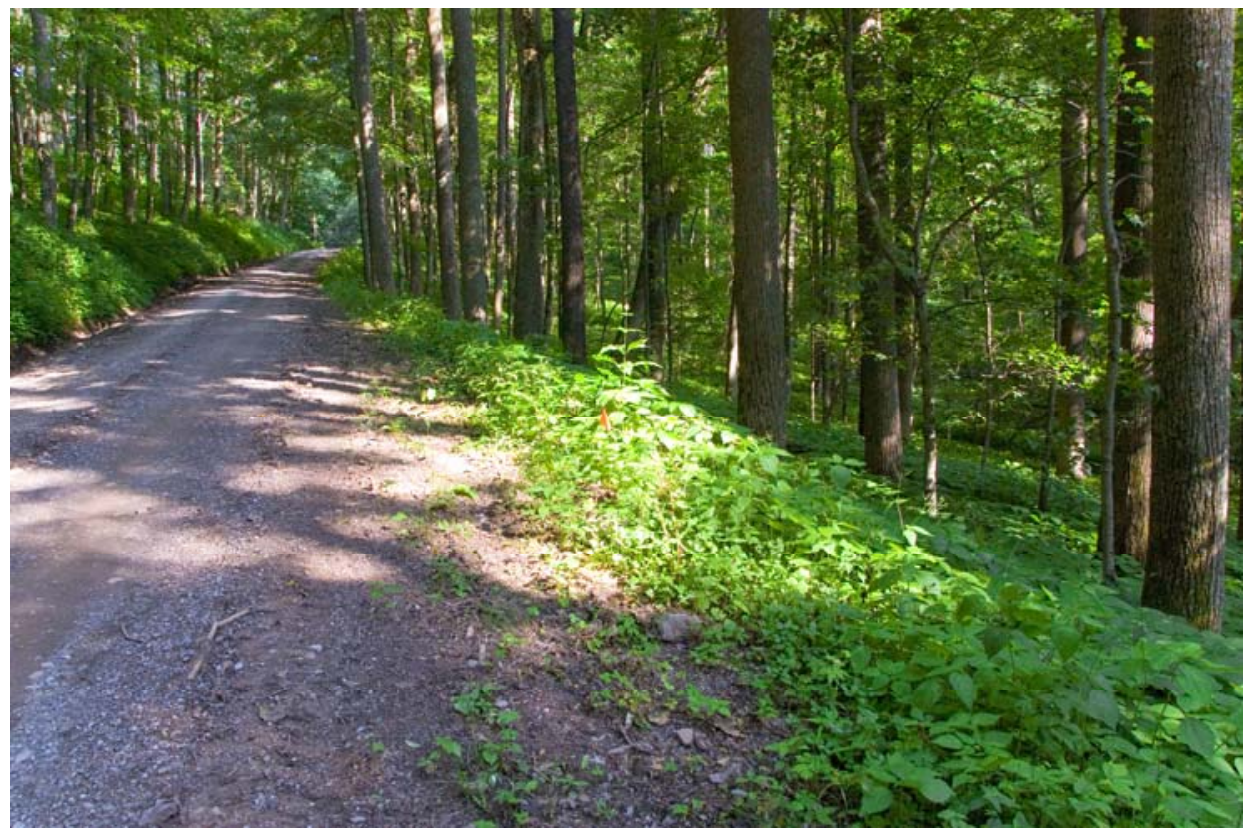

\section{Trifolium stoloniferum growing along improved road.}

This is a picture of T. stoloniferum growing successfully at BSG6 with multiple flowers visible in the picture. There were 178 rooted crowns and 56 inflorescences, giving the site a flowering index of 0.31 , which was very good. Later in the 2009 season, this site was crushed by a vehicle.

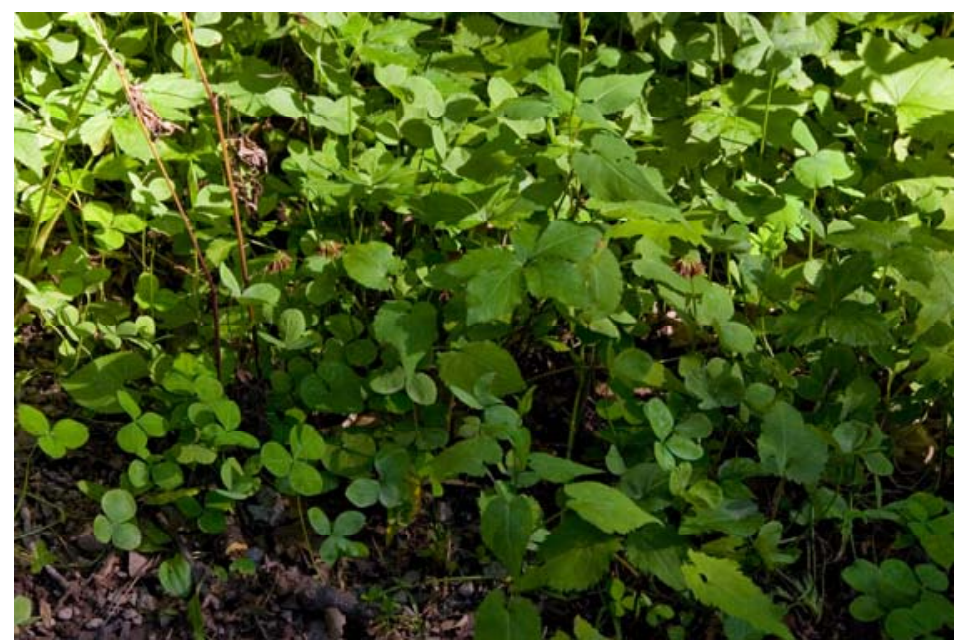

Flowering T. stoloniferum. 
The light environment at BSG6 was very favorable to flowering- $12.54 \mu \mathrm{mol}$ photons $/ \mathrm{m}^{2} / \mathrm{second}$ - which is slightly above the mean of the most successfully flowering sites (a.k.a. Flowergroup 3)- $12.12 \mu \mathrm{mol}$ photons $/ \mathrm{m}^{2} / \mathrm{second}$ (SD 4.07).

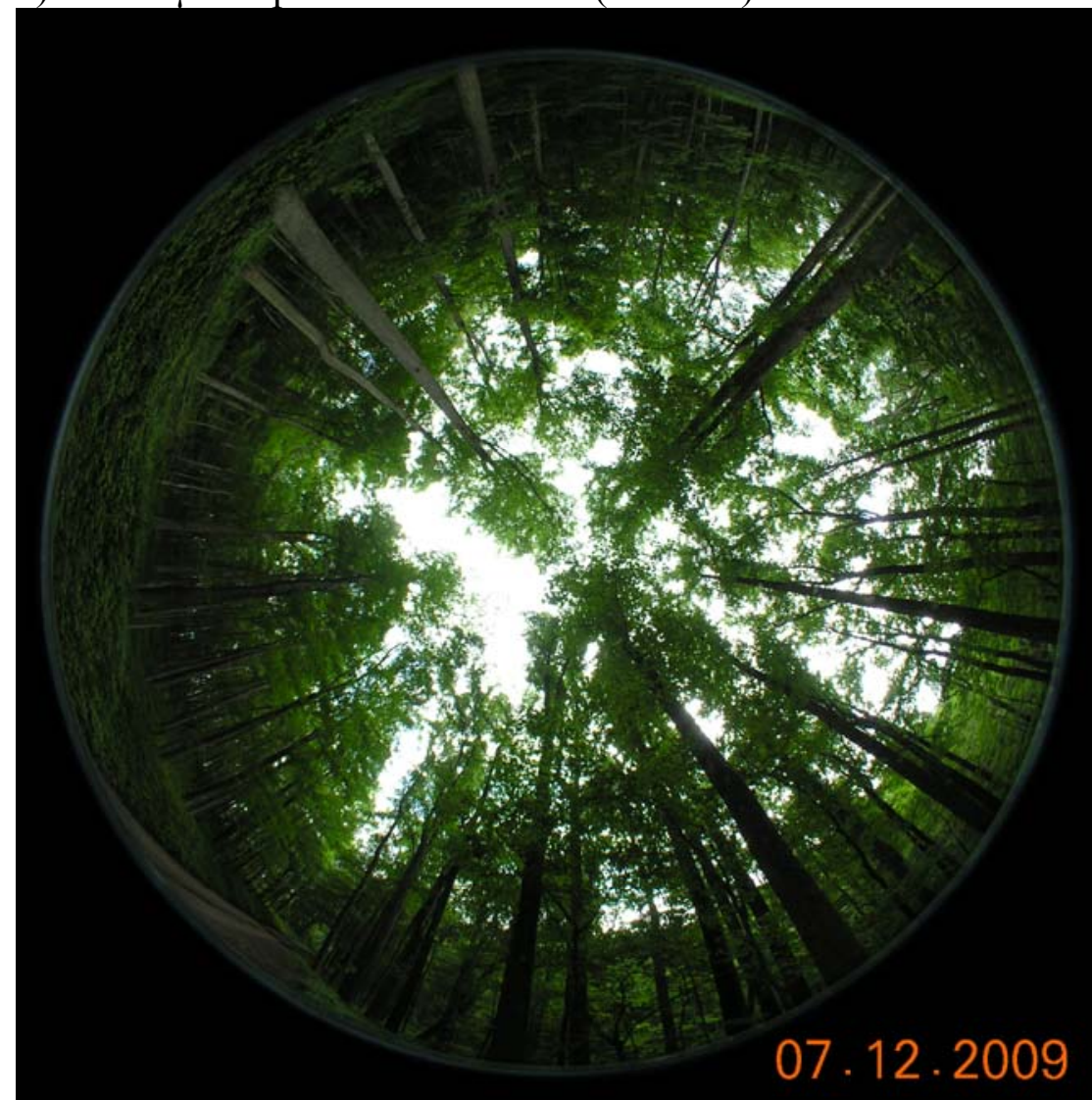

Canopy structure of a successful T. stoloniferum site. 


\section{3) Patch 9-19: Crowngroup 3, Flowergroup 2}

9-19 had the highest abundance of any site-565 rooted crowns-and relatively high reproductive success-108 inflorescences for a flowering index of 0.19 (putting it into Flowergroup 2). This site was characterized by its steep (and slippery!) slope and extremely high plant diversity. Shannon's index was 3.22 at this patch, which was the highest recorded at any site (overall mean among all sites of Shannon's index was 2.67 (SD 0.36)).

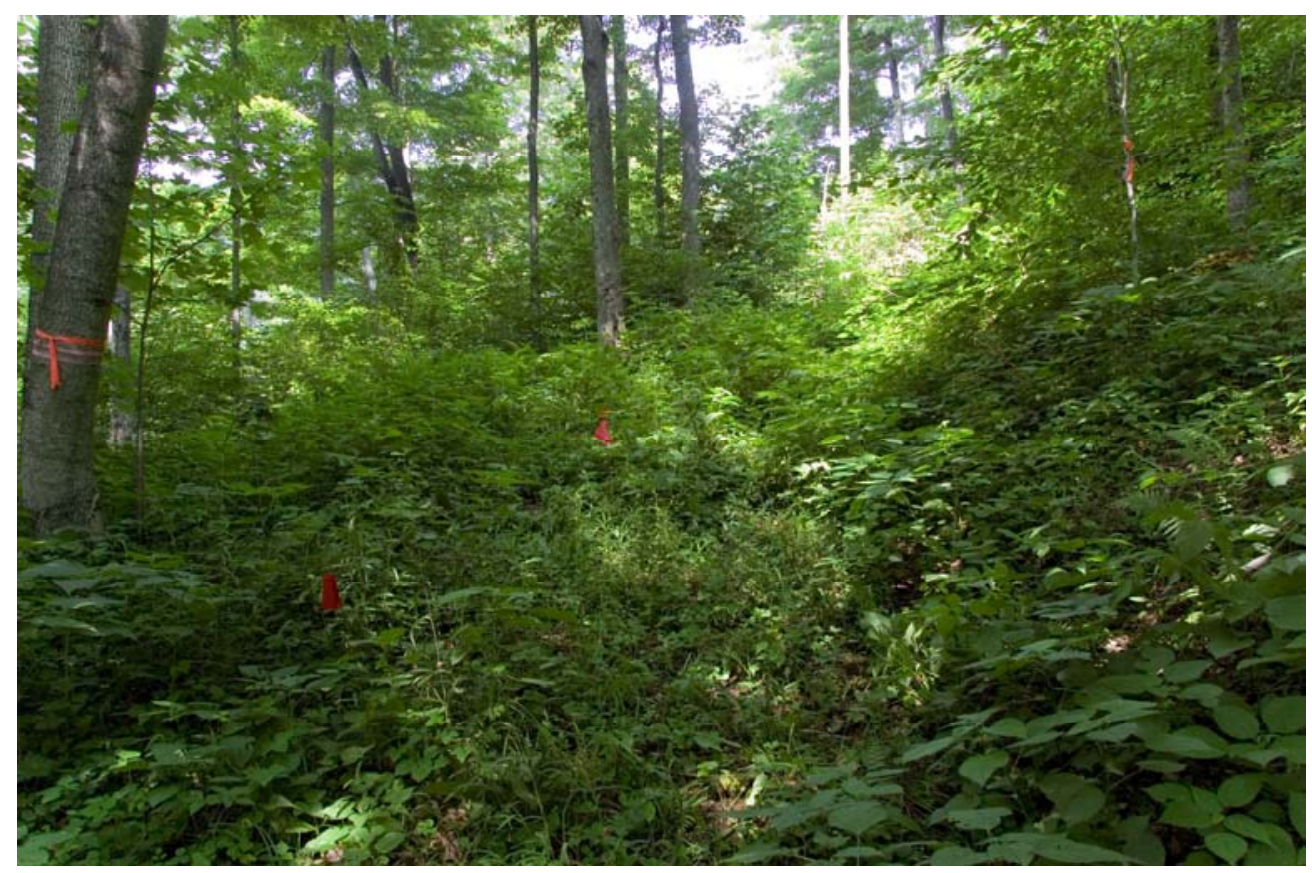

Vegetation looking uphill.

Total vegetative cover was $76.4 \%$, which was relatively high. Time since last disturbance was 7 years.

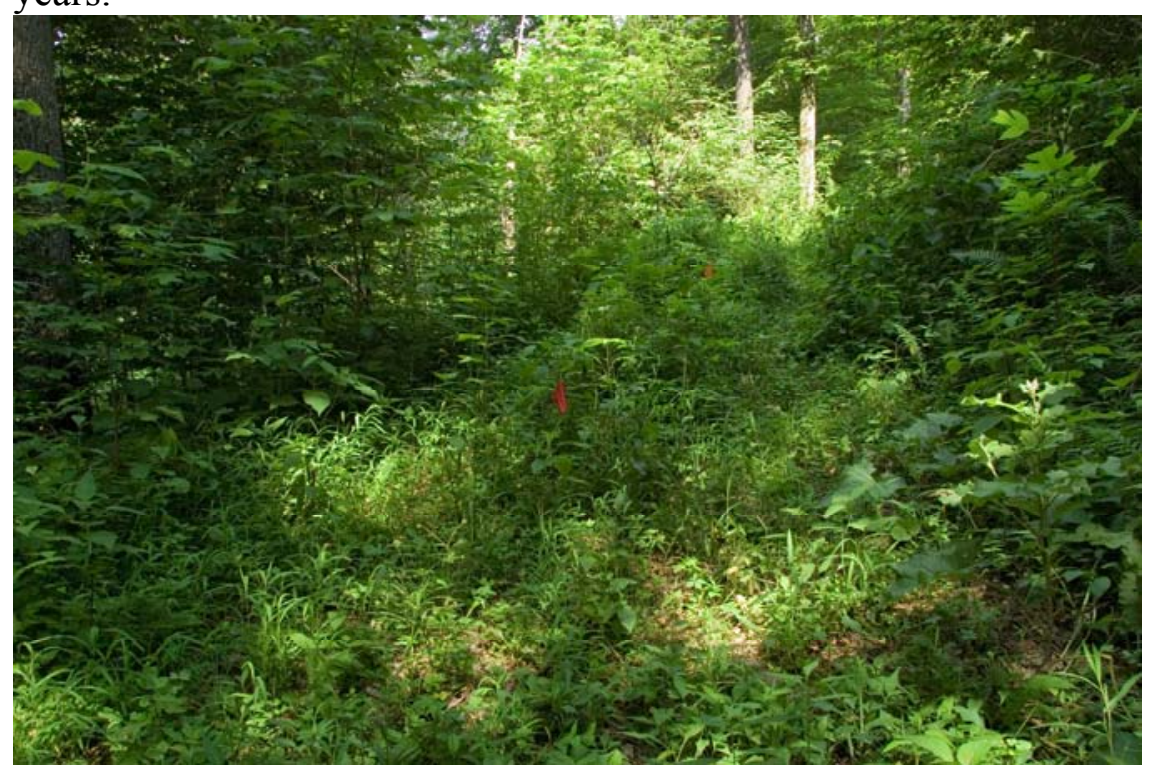

Vegetation looking downhill. 
Measured light levels at this site were below the Crowngroup 3 mean (9-19 received $8.15 \mu$ mol photons $/ \mathrm{m}^{2} /$ second and the group mean was 9.53 ). Thus, the slightly lower reproductive success at this site could be attributed to sub-optimal light conditions, as compared to 9-46 or BSG6. Still, this site could be characterized as a very successful site. Perhaps the fertility of this site which was responsible for the high diversity made up for the sub-optimum light levels and allowed T. stoloniferum to thrive.

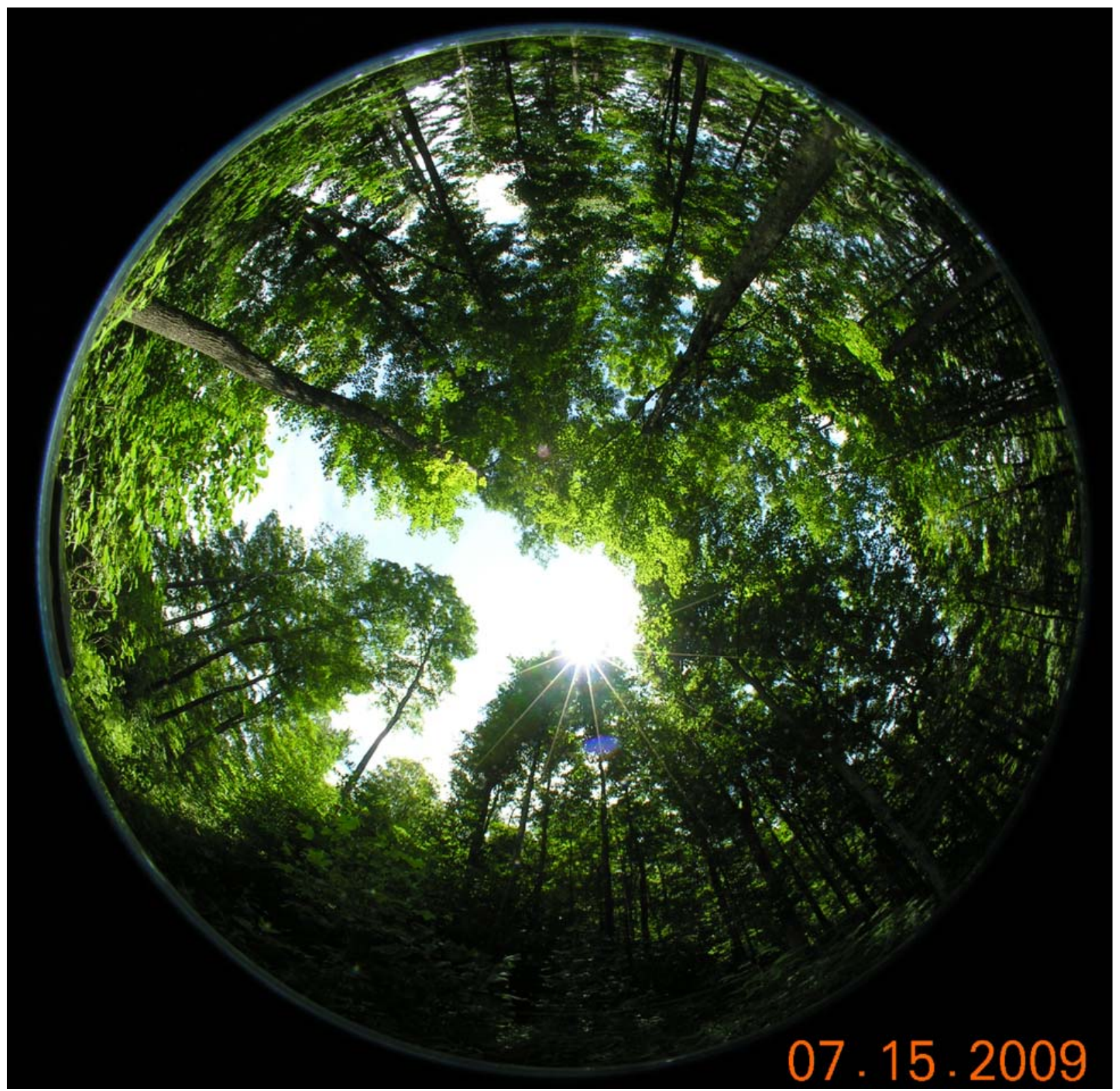

Canopy structure of a successful T. stoloniferum site. 
Next I present a site that has high abundances of $T$. stoloniferum rooted crowns but low reproductive success.

\section{4) Patch 9-52/53: Crowngroup 3, Flowergroup 1}

Patch $9-52 / 53$ is characterized by extremely low light levels $\left(3.87 \mu \mathrm{mol}\right.$ photons $\left./ \mathrm{m}^{2} / \mathrm{second}\right)$, and consequently, low reproductive success. There were 125 rooted crowns in this patch, but only 2 inflorescences. This extremely low rate of inflorescence production suggests that light is limited at this site. Perhaps at some point in the past, maybe shortly after it was disturbed 7 years ago, the light environment was more favorable and promoted reproduction.

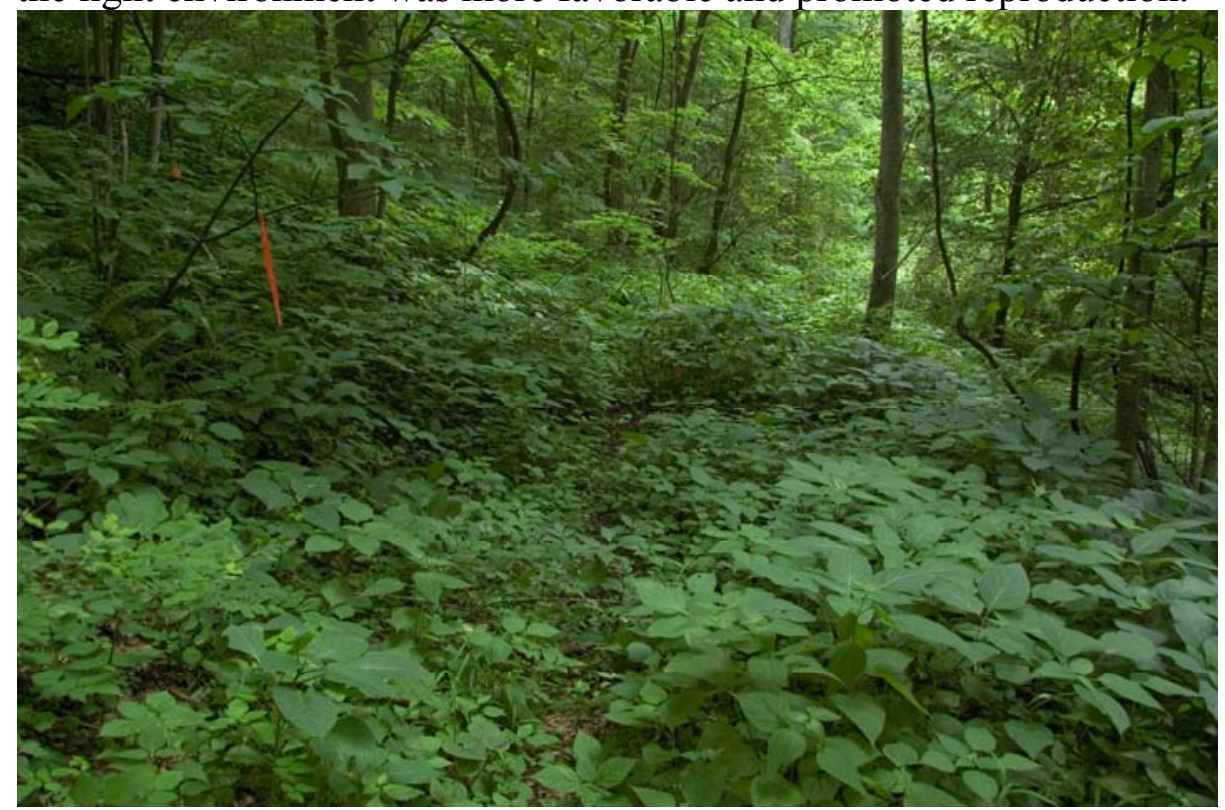

Vegetation of a high abundance, low flowering site. 
The canopy structure at 9-52/53 is more closed than other sites with abundant crowns. Canopy openness is $5.35 \%$, which is about $1 \mathrm{SD}$ below the mean of Crowngroup 3. Additionally, measured light levels were the lowest among all Crowngroup 3 sites at $3.87 \mu \mathrm{mol}$ photons $/ \mathrm{m}^{2} /$ second.

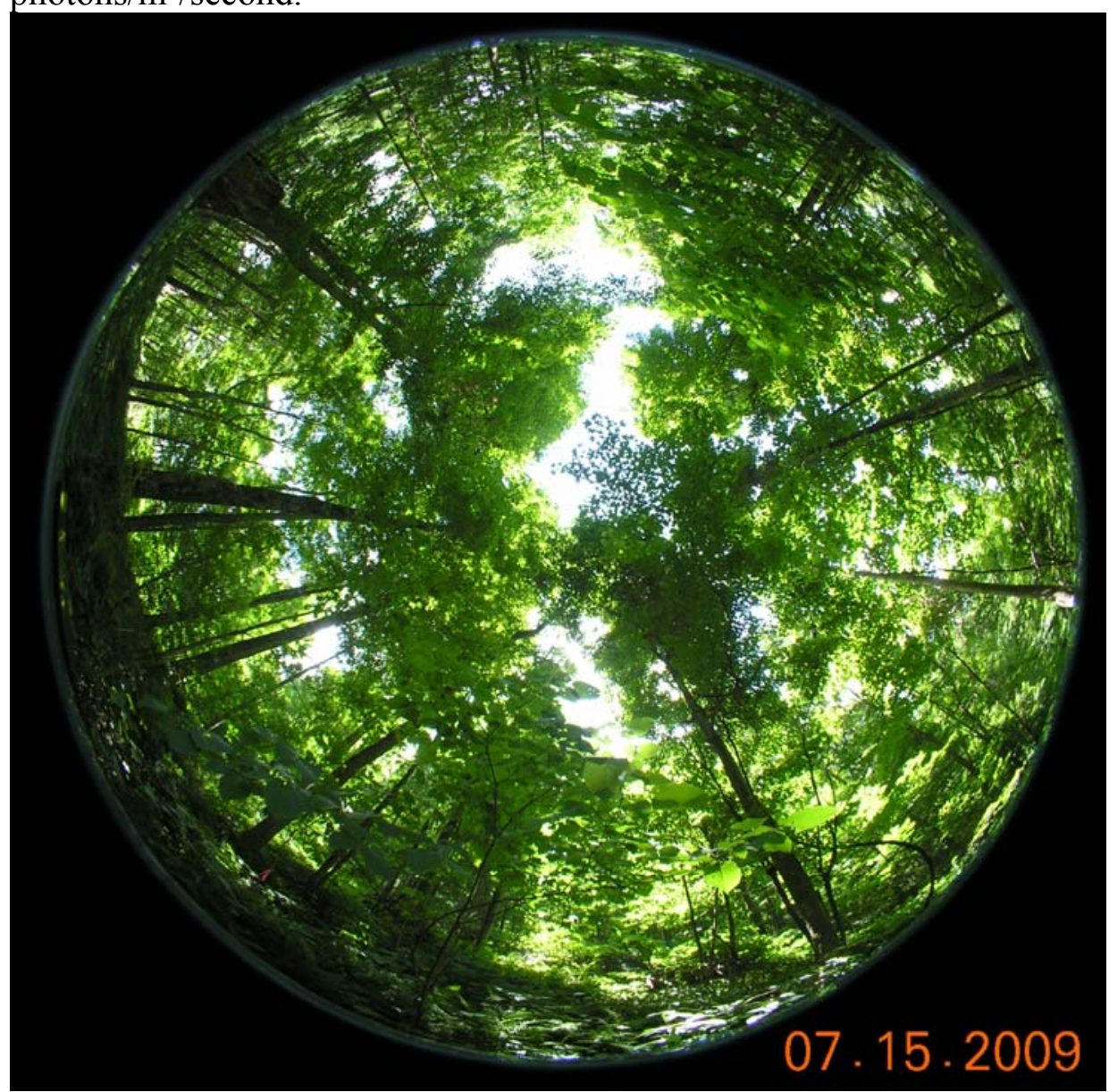

Canopy structure of a low flowering site. 
Next, we will describe some sites from Crowngroup 2, or those sites with 30-99 rooted crowns. These sites vary widely in their relative reproductive success.

\section{5) Patch 9-36: Crowngroup 3, Flowergroup3}

Patch 9-36 is characterized by high reproductive success (Flowering index of 0.64), very high shrub coverage (14.7 meters compared to Crowngroup 2 mean of 3.46), and a high sapling tally (39 compared to Crowngroup 2 mean of 24.56 (SD 15.08)).

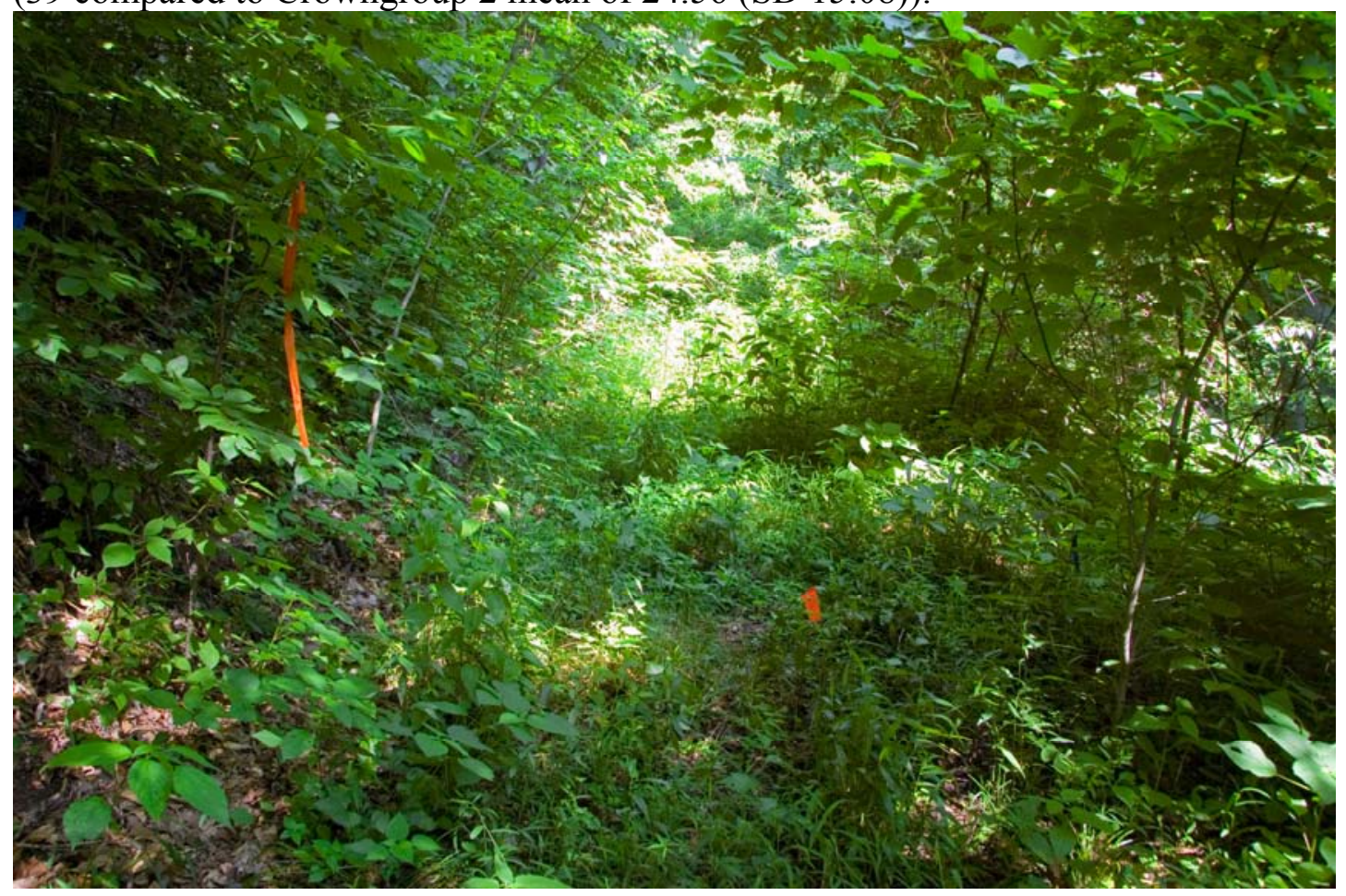

Vegetation of a medium-abundance site. 
Patch 9-36 has light levels close to the Crowngroup 2 mean $\left(8.74 \mu \mathrm{mol}\right.$ photons $/ \mathrm{m}^{2} / \mathrm{second}_{\mathrm{vs}}$. 8.64 (SD 3.17)) and canopy openness lower than the group mean (8.04\% vs. 9.53\%).

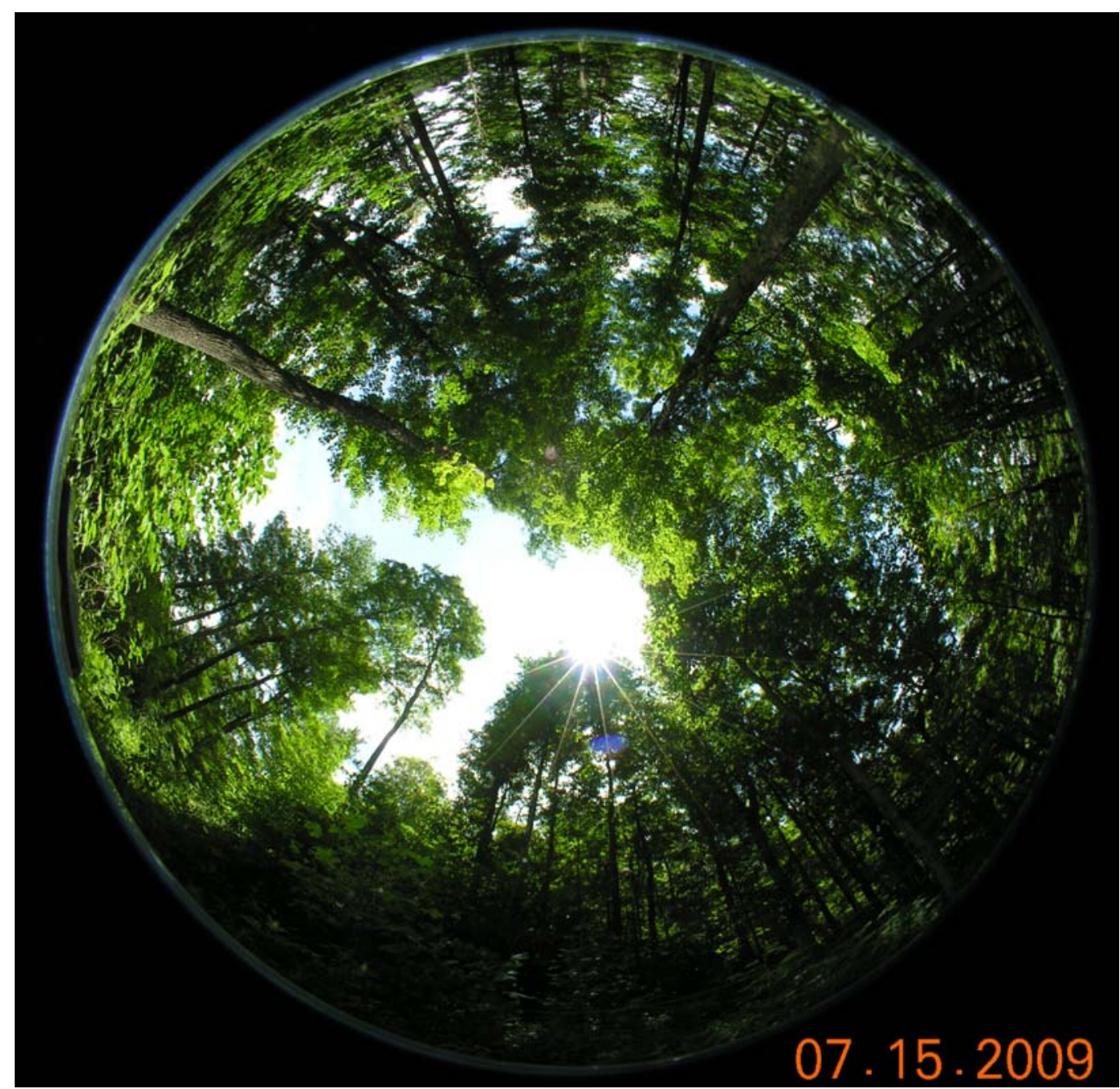

Canopy structure of a high-flowering site. 


\section{6) Patch 20A-e: Crowngroup 2, Flowergroup 3}

Patch 20A-e is characterized by very high light levels $\left(15.4 \mu \mathrm{mol}\right.$ photons $\left./ \mathrm{m}^{2} / \mathrm{second}\right)$ and relatively high levels of $T$. stoloniferum reproductive success (flowering index of 0.31 ). Unfortunately I don't have a photograph of vegetation this site. The T. stoloniferum sites of Compartment 20 had a distinct flora, which in my impression was distinguished from other sites by abundant Hypericum punctatum.

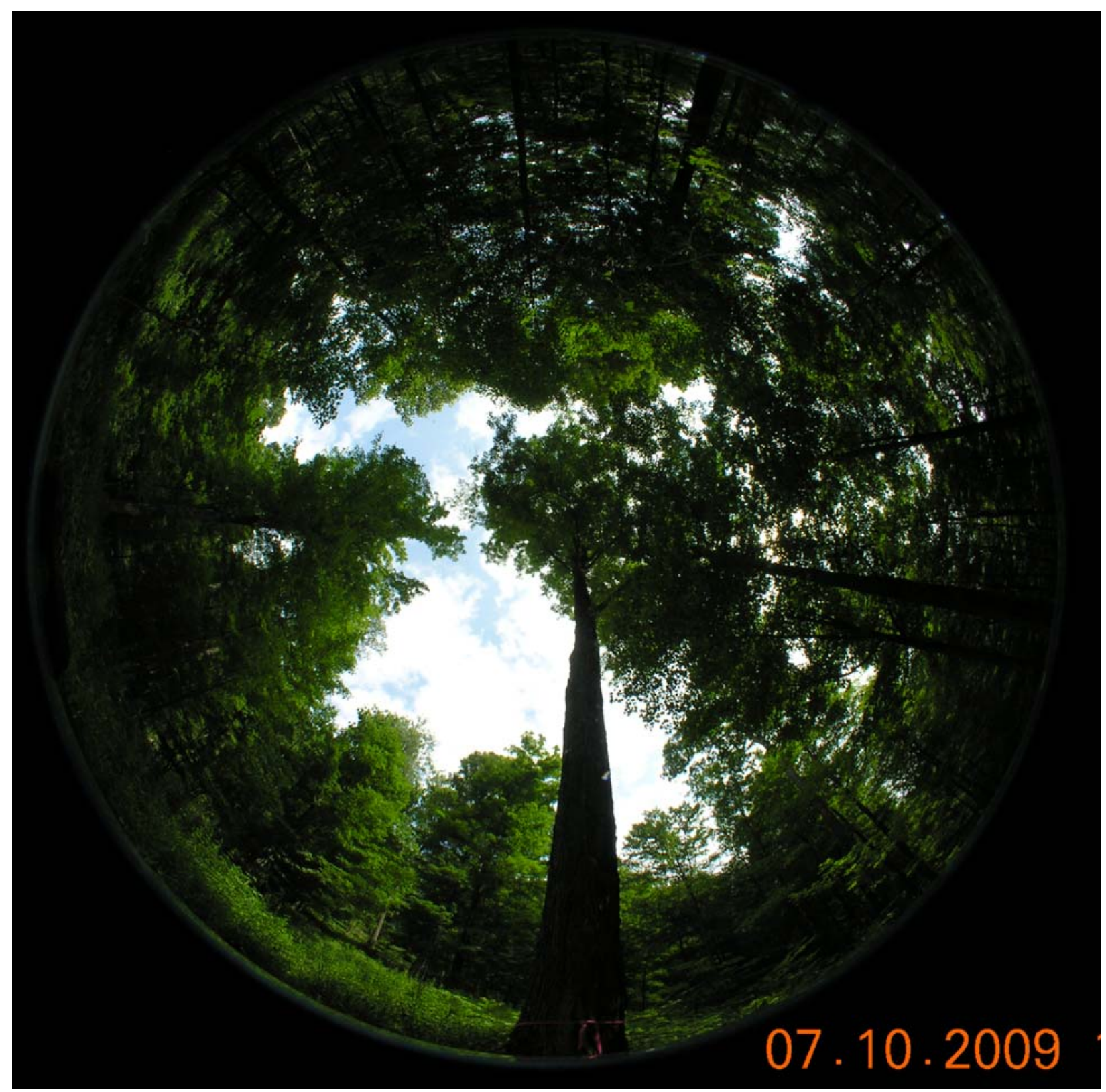

Canopy structure of a high-flowering site. 


\section{7) Patch 9-33: Crowngroup 2, Flowergroup 2}

9-33 is a site with a medium-to-low level of reproductive success (60 crowns and 8 inflorescences for a flowering index of 0.13 ). This site appears to be average in many ways with respect to Crowngroup 2: total understory cover $=64.5 \%$ (Crowngroup 2 mean $=64.9 \%$ ), shrub cover (5.6m vs. Crowngroup 2 mean=3.46), and a time since last disturbance of 7 years (Crowngroup 2 mean=8.88).

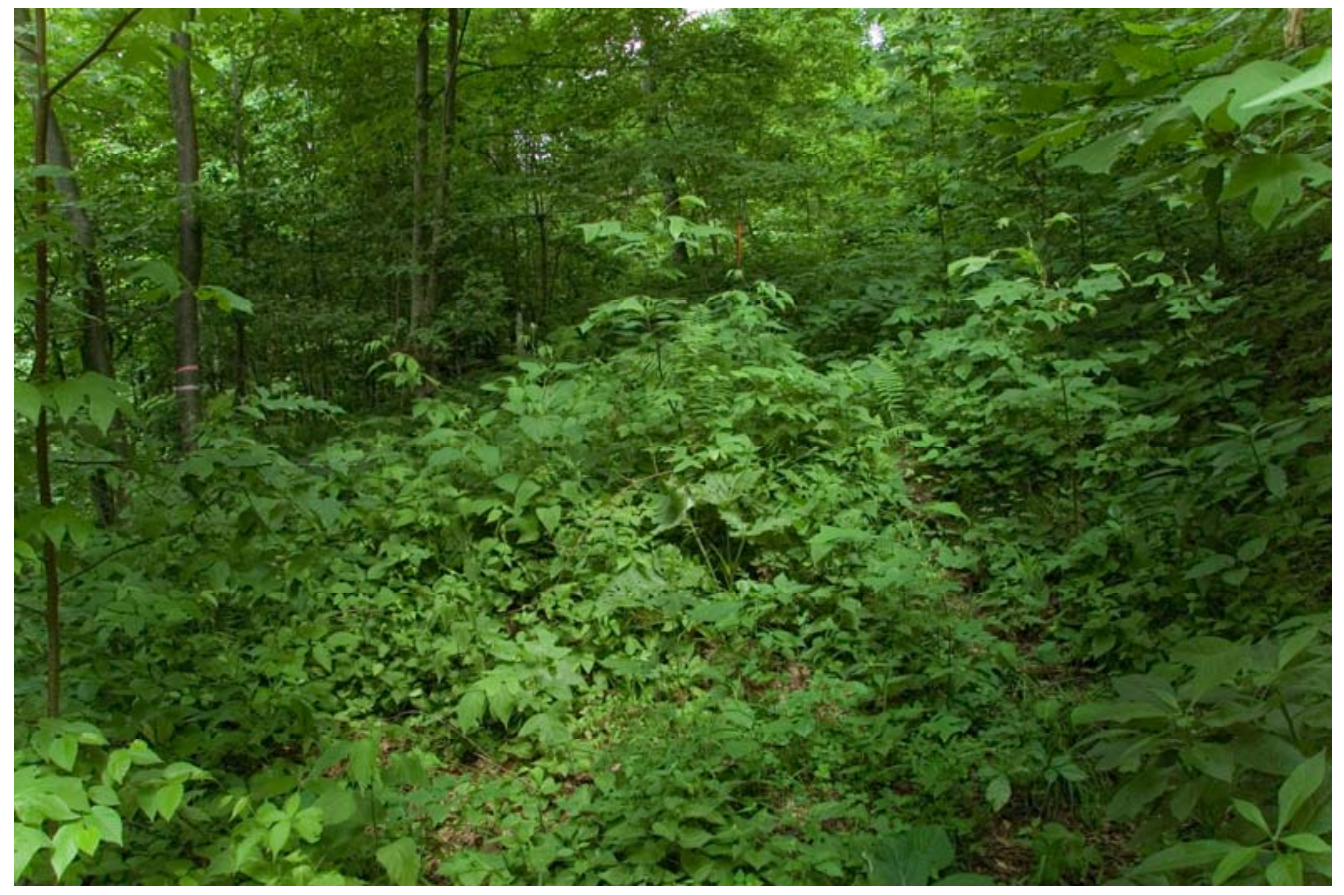

Vegetation

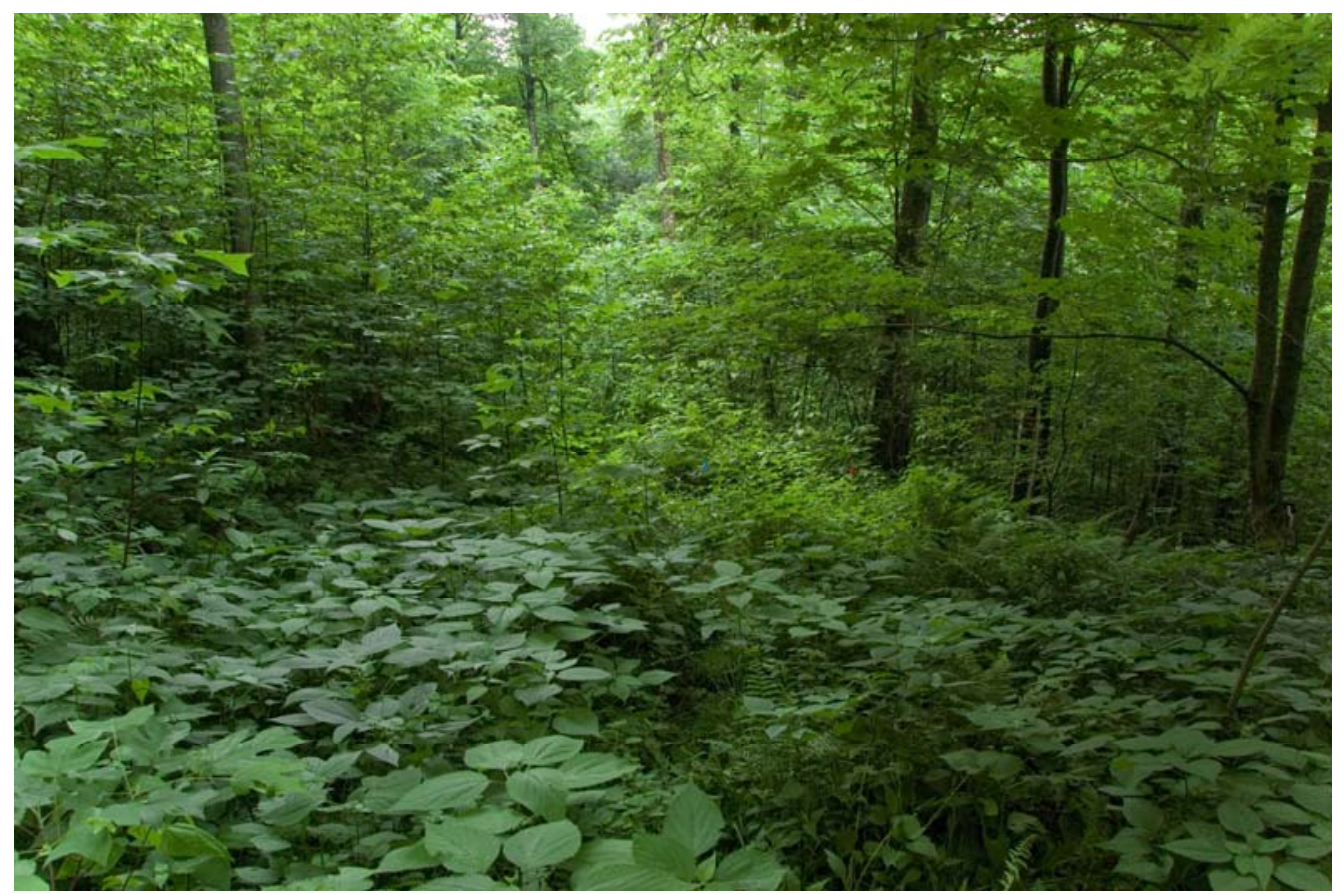

Vegetation 
But the relative reproductive success of patch 9-33 is lower than the mean of Crowngroup 2 sites (FI of 9-33 was 0.13 and the mean of Crowngroup 2 was 0.22 ). This is most likely because it has lower canopy openness (7.26 vs. Crowngroup 2 mean of 9.53) and photosynthetically active radiation $\left(6.36 \mu \mathrm{mol}\right.$ photons $/ \mathrm{m}^{2} /$ second vs. Crowngroup 2 mean of 8.64$)$. In short, 9-33 is a mediocre site for T. stoloniferum mainly because of the low light levels.

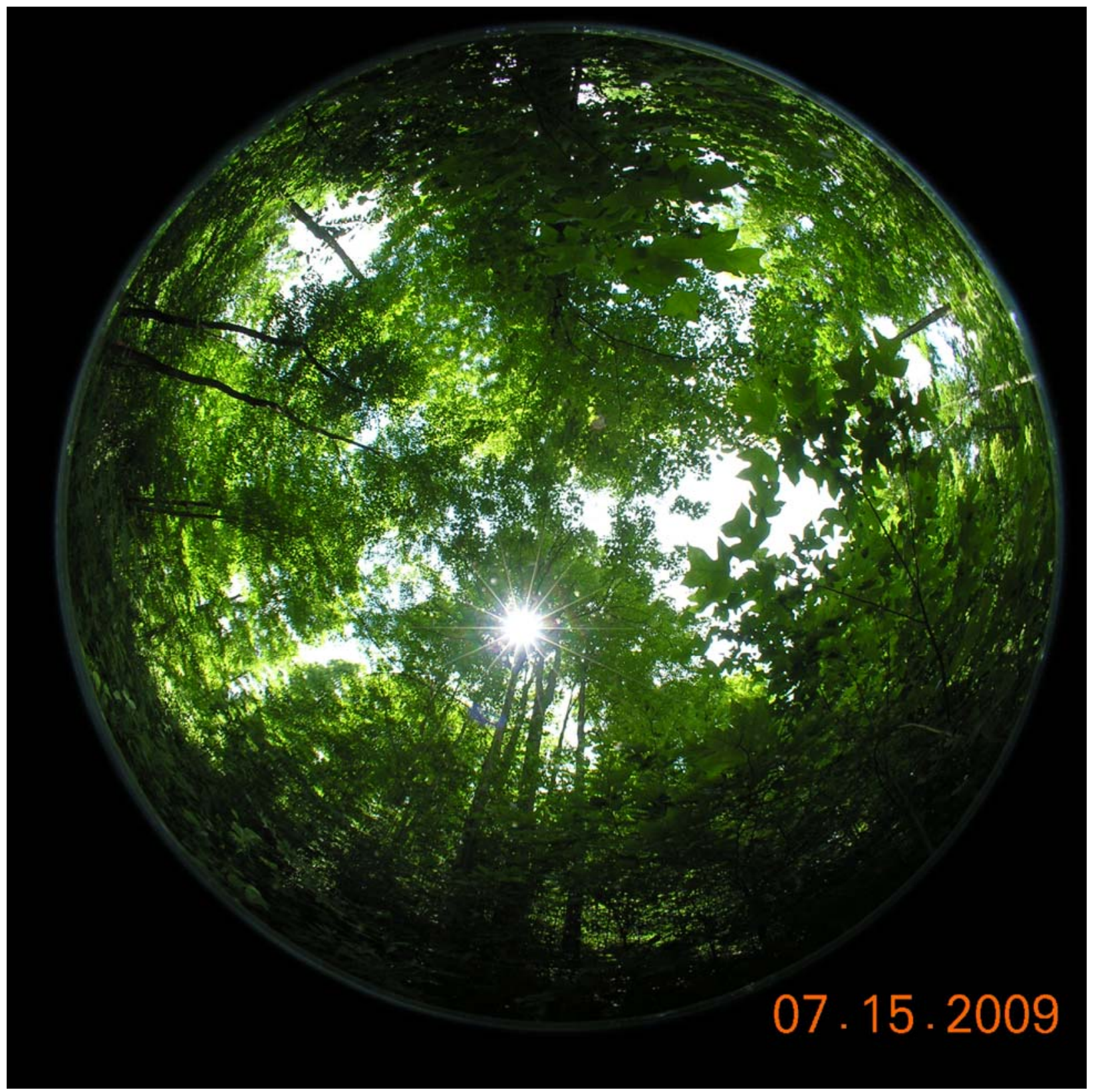




\section{8) Patch 16-6:}

Patch 16-6 exemplifies a declining T. stoloniferum site. There was a very high number of saplings, particularly of Liriodendron tulipifera.

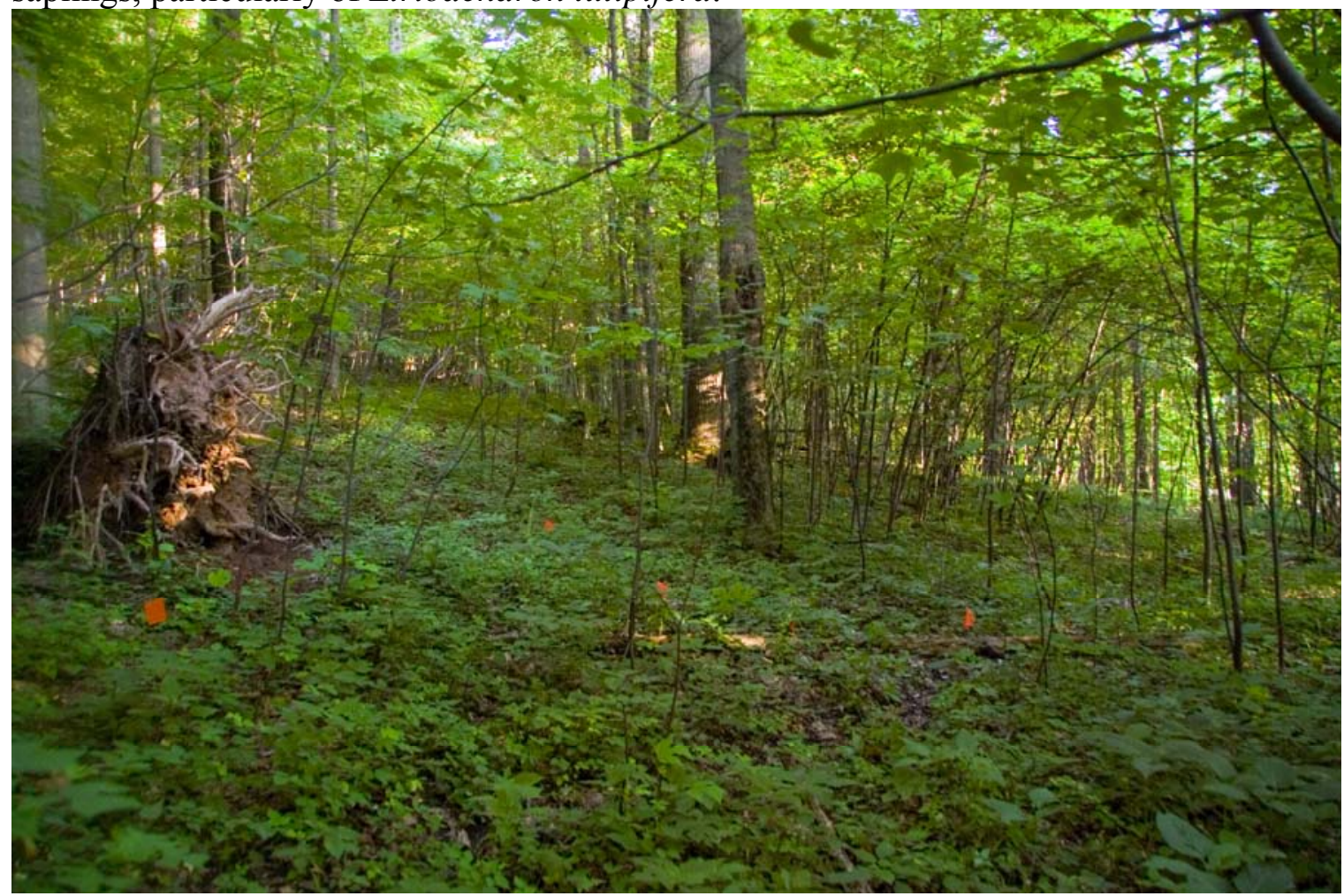

Vegetation exemplifying the high number of tree saplings found at this site. 
The light levels and canopy openness were not low $\left(8.18 \mu \mathrm{mol}\right.$ photons $/ \mathrm{m}^{2} / \mathrm{second} \mathrm{vs}$. Crowngroup 2 mean of 8.64 and $12.46 \%$ canopy openness vs. 9.53\% mean of Crowngroup 2). It appears that the long time since last disturbance (19 years) has allowed for the reinitiation of trees in the understory. The trees appear to be capturing a substantial proportion of the light resources which inhibits $T$. stoloniferum from reproducing successfully.

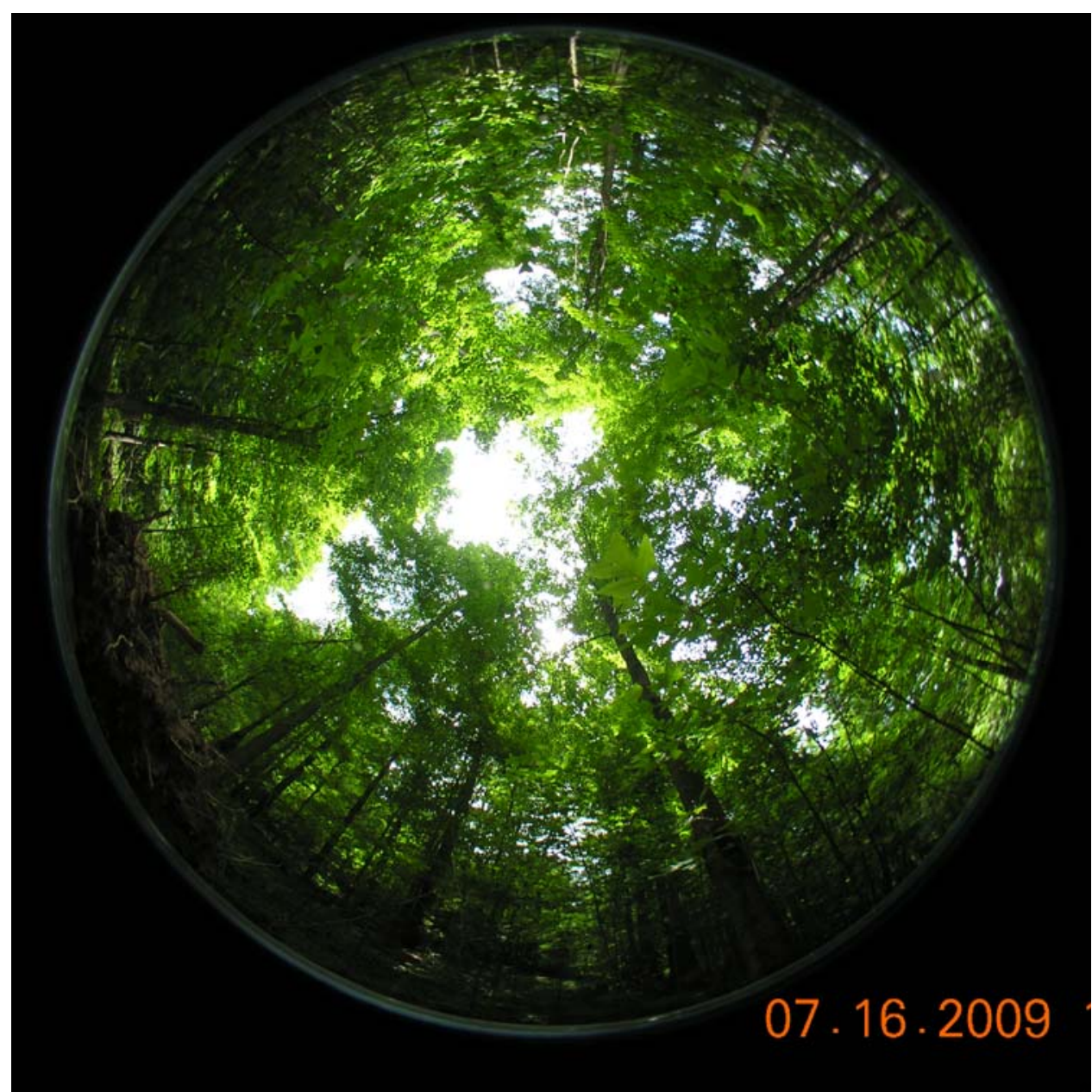

Canopy of a declining site 
Now, I present to you some of the sites that had low abundances of T. stoloniferum rooted crowns (Crowngroup 1: 1-30 rooted crowns). These sites had varying levels of reproductive success.

\section{9) Patch 13A-1}

Patch 13 A-1 exemplifies the up-and-coming T. stoloniferum site. The site had the highest flowering index of any other site $(\mathrm{FI}=1.69)$. There were 16 robust crowns and 27 inflorescences in the patch. Total vegetative cover in the understory was lower than the mean for Crowngroup $1(52.1 \%$ vs. $62.82 \%$ (SD 14.77) as the mean of Crowngroup 1). Time since last disturbance was six years, less than the mean for Crowngroup 1 (9.83 years (SD 11.26)). The basal area $90 \mathrm{ft}^{2} / \mathrm{ac}$ is not substantially higher than the mean $81 \mathrm{ft}^{2} / \mathrm{ac}$ (SD 26.1) for Crowngroup 1. The sapling tally for this plot was not different from the mean for Crowngroup 1. Shannon's diversity index is lower than the mean for Crowngroup 1 (2.45 vs. mean of 2.63 for Crowngroup 1).

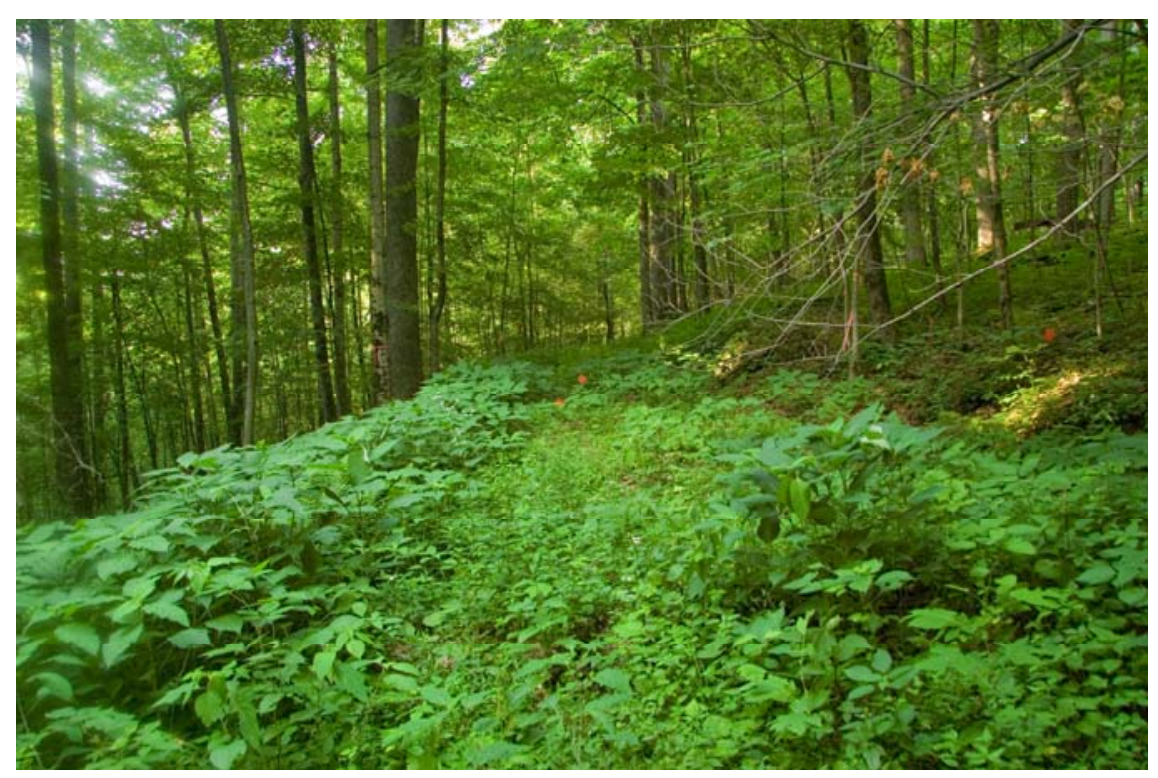

Vegetation of an up-and-coming site. 
The light environment appears to be particularly favorable for T. stoloniferum at 13A-1. Photosynthetic photon flux density is $14.79 \mu \mathrm{mol}$ photons $/ \mathrm{m}^{2} / \mathrm{second}$, which is higher than the Crowngroup 1 mean $7.63 \mu \mathrm{mol}$ photons $/ \mathrm{m}^{2} /$ second (SD 5.70).

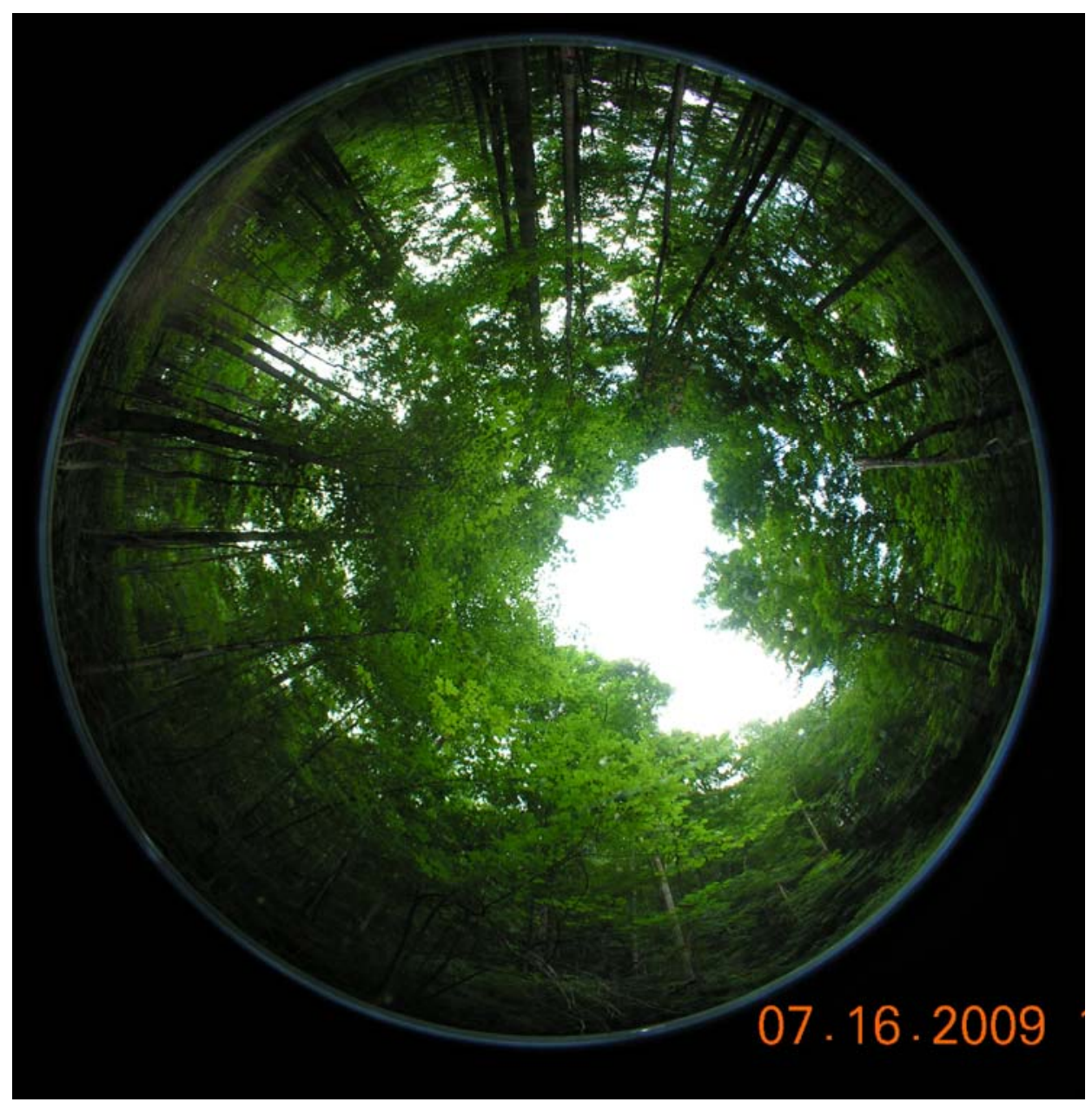

Canopy of a vigorous young T. stoloniferum patch. 


\section{0) BSG7G}

BSG7G had 76 rooted crowns and 5 inflorescences, and the flowering index was 0.07 . Overall, the reproductive success of this patch was relatively low. The question is "why"?

This site was recovering from a disturbance that had occurred only 1-2 years previous. The basal area of the site was relatively low- $60 \mathrm{ft}^{2} / \mathrm{ac}$, which was below the mean for Crowngroup $1(80.8$ $\left.\mathrm{ft}^{2} / \mathrm{ac}\right)$. Total vegetative cover was not substantially different from the mean for Crowngroup 1 $(68.2 \%$ cover vs. Crowngroup 1 mean of $62.82 \%)$. Understory diversity is higher than at other Crowngroup 1 sites (2.92 vs. Crowngroup 1 mean of 2.63).

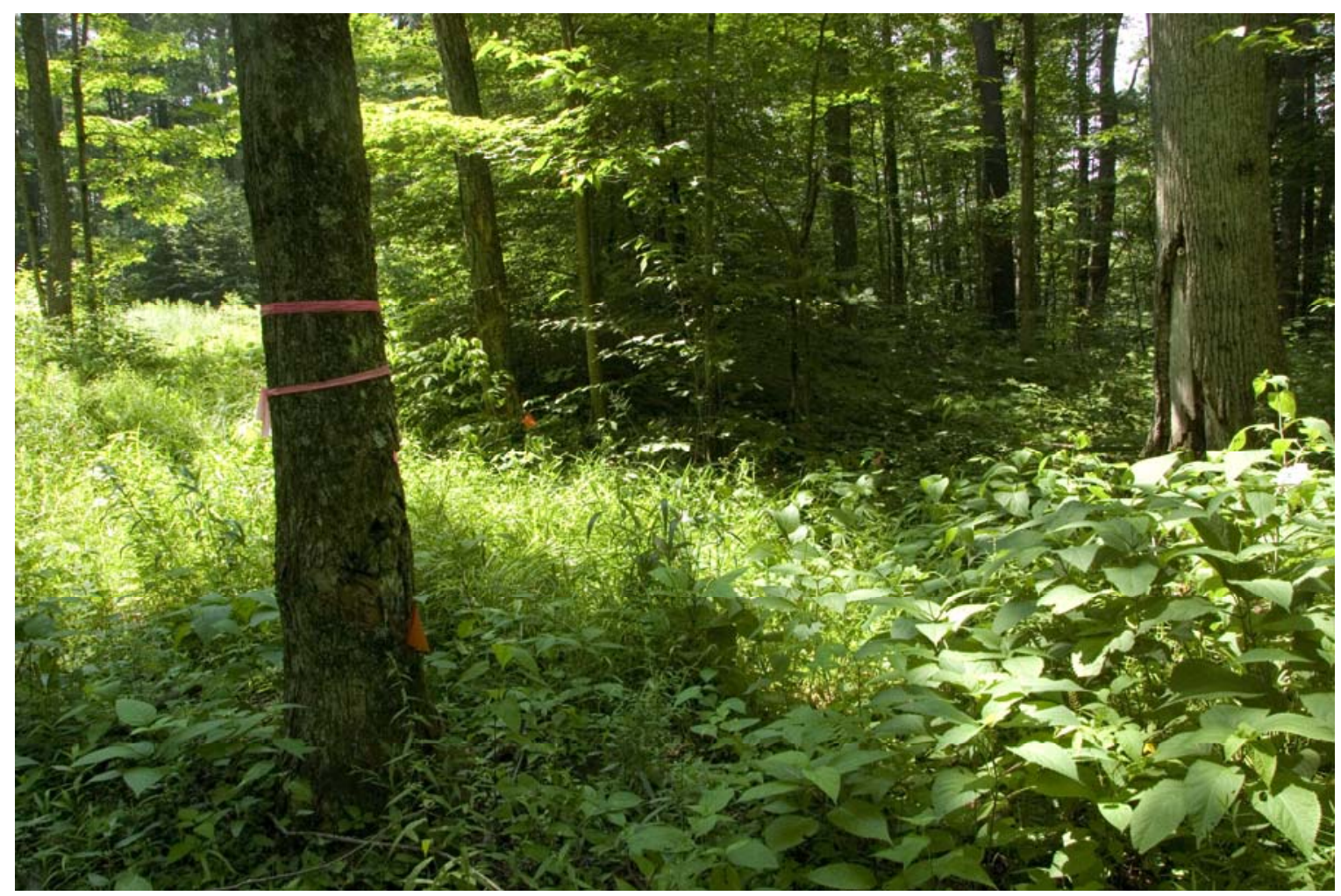

\section{Vegetation at BSG7G}

The light environment was not optimal- $7.72 \mu \mathrm{mol}$ photons $/ \mathrm{m}^{2} /$ second - but slightly above the Crowngroup 1 mean $\left(7.63 \mu \mathrm{mol}\right.$ photons $\left./ \mathrm{m}^{2} / \mathrm{second}\right)$. It is difficult to say conclusively why this site is not flowering prolifically, but it is likely a function of the recent disturbance, sub-optimal light environment and perhaps competition from other plants. 
Burkhart 134

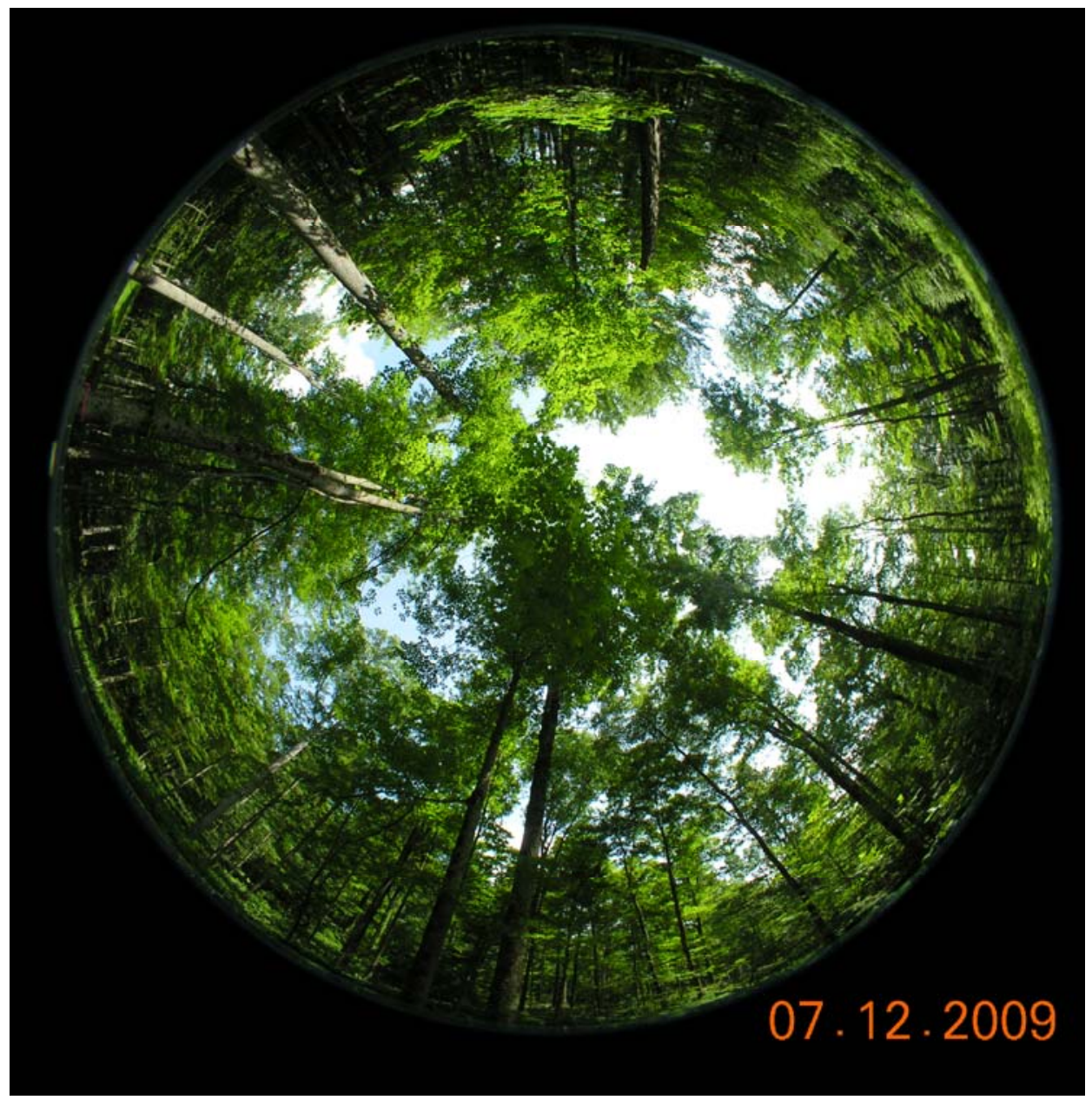

Canopy at BSG7G, whose light environment was sub-optimal. 


\section{1) Patch BSG2-B}

BSG2-B was one of the sites at which T. stoloniferum was first found at the Fernow Experimental Forest in 1999 and has been monitored since that time. The abundance of BSG2-B peaked in 2005 at 106 rooted crowns (counts of inflorescences were not made, but Dr. Thomas Schuler recalls profuse flowering at this site around that time). As of 2009, there were 15 crowns at this site and no inflorescences. This site has not been disturbed by logging or other vehicular traffic in many years (last recorded disturbance was 33 years ago), but foot traffic to visit a spring causes frequent small trampling events. Basal area is moderately high at the site (110 $\mathrm{ft}^{2} /$ ac compared to Crowngroup 1 mean of $80.8 \mathrm{ft}^{2} / \mathrm{ac}$ (SD 26.1) and understory vegetative cover is close to average (59.2\% compared to $62.82 \%$ (SD 14.77). Diversity was relatively low at 2.16 Shannon's Index, compared to the Crowngroup 1 mean of 2.63, and the site was dominated by Symphyotrichum prenanthoides, crooked-stem aster, and Laportea canadensis, wood-nettle.

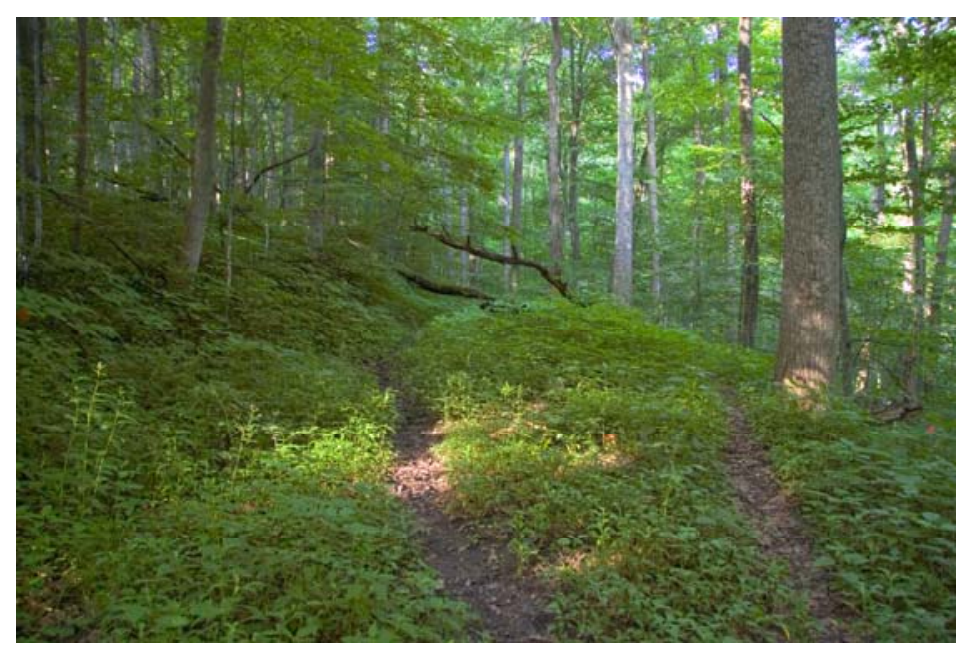

Vegetation at BSG2-B, exemplifying a declining site.



Vegetation as BSG2-B with dense Laportea canadensis cover. 
The light and canopy conditions were not favorable for growth or inflorescence production of $T$. stoloniferum. Percent canopy openness was very low, at $5.73 \%$ compared to Crowngroup 1 mean of 10.08. Light levels were also quite low- $3.72 \mu \mathrm{mol}$ photons $/ \mathrm{m}^{2} / \mathrm{second}$ compared to Crowngroup 1 mean of 7.63. The dark conditions of this site most likely have contributed to the decline of this patch.

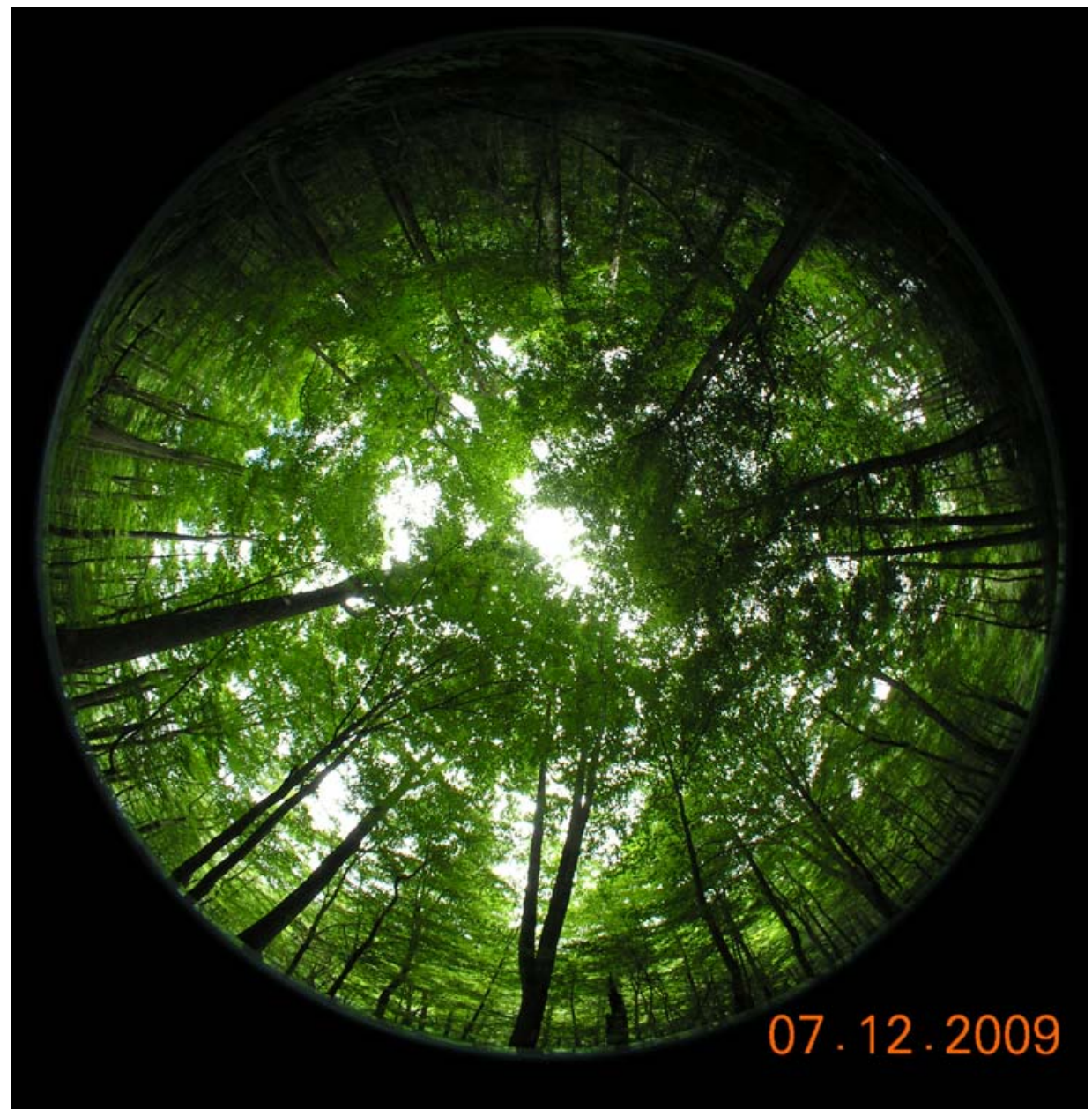

Canopy of BSG2-B 
Next, I provide examples of control sites. Patch 16-1, Patch 17A, and Patch BSG5 all contained T. stoloniferum in the past, but it is no longer found at any of these sites. Trifolium stoloniferum has never been found at Control 14-2. This site was selected to serve as a control because it was situated along a skid road in a mature forest underlain by calcareous soil, but it differed in disturbance history from those sites that contained T. stoloniferum

\section{1) Patch 16-1}

Patch 16-1 is located along a skid road downhill from 16-6, which is a declining site I showed earlier. Like 16-6, 16-1 has a relatively high sapling tally (29 compared to Crowngroup 0 mean of 22.64), very low vegetative cover (33.66\% compared to Crowngroup 0 mean of $47.60 \%$ ) and as can be seen from the photograph, a thick leaf litter layer. This site had not been disturbed by logging for 19 years, which is similar to the mean of all Crowngroup 0 sites (19.64 years SD $(19.10))$.

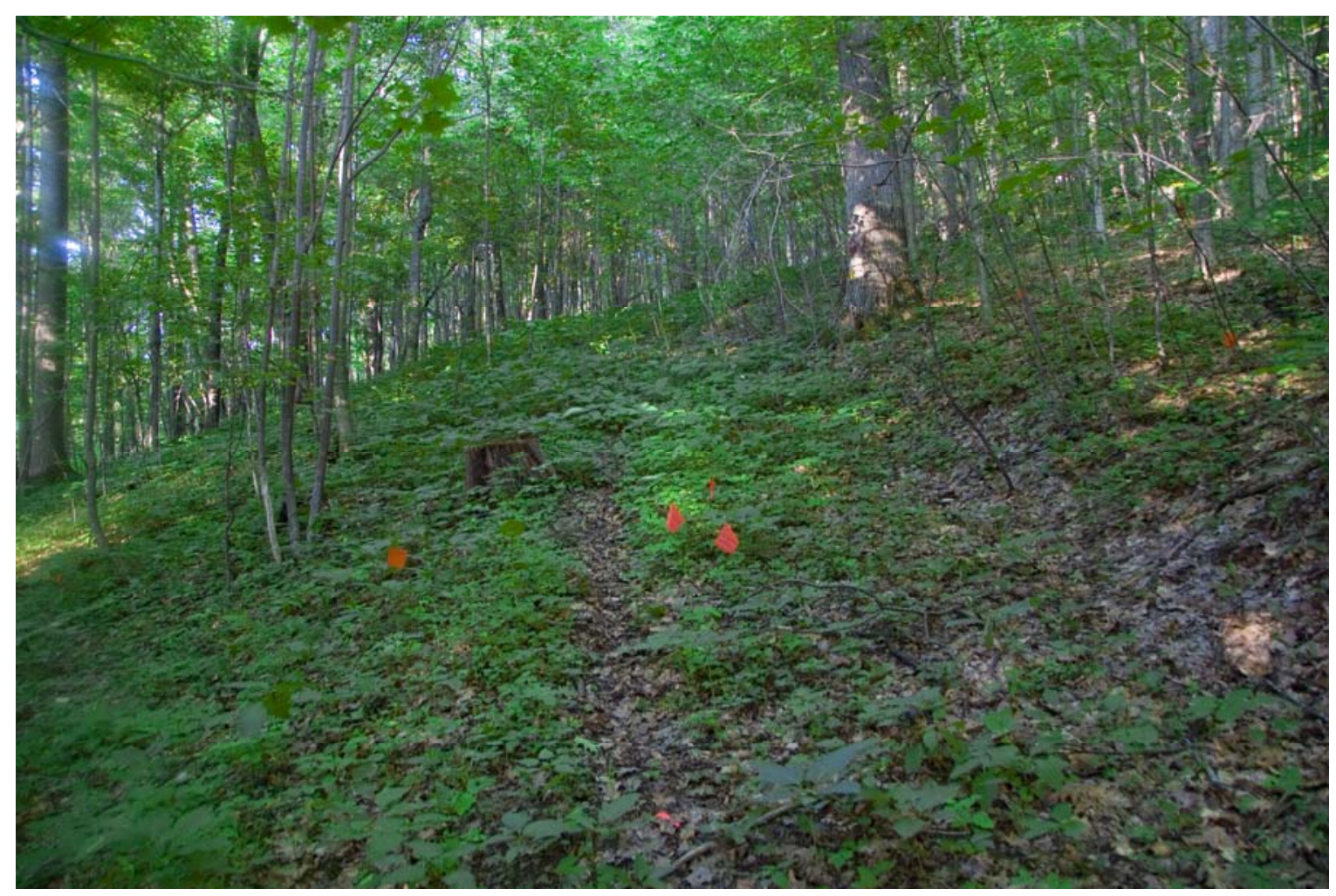

Vegetation at Patch 16-1 
The light environment of 16-1 appears to be unfavorable to inflorescence production and growth. The photosynthetically active radiation reaching the herb layer is $3.82 \mu \mathrm{mol}$ photons $/ \mathrm{m}^{2} / \mathrm{second}$. The combination of canopy closure and limited to no leaf litter/soil disturbance have contributed to the decline of $T$. stoloniferum at this site and prevented $T$. stoloniferum from reestablishing at the site.

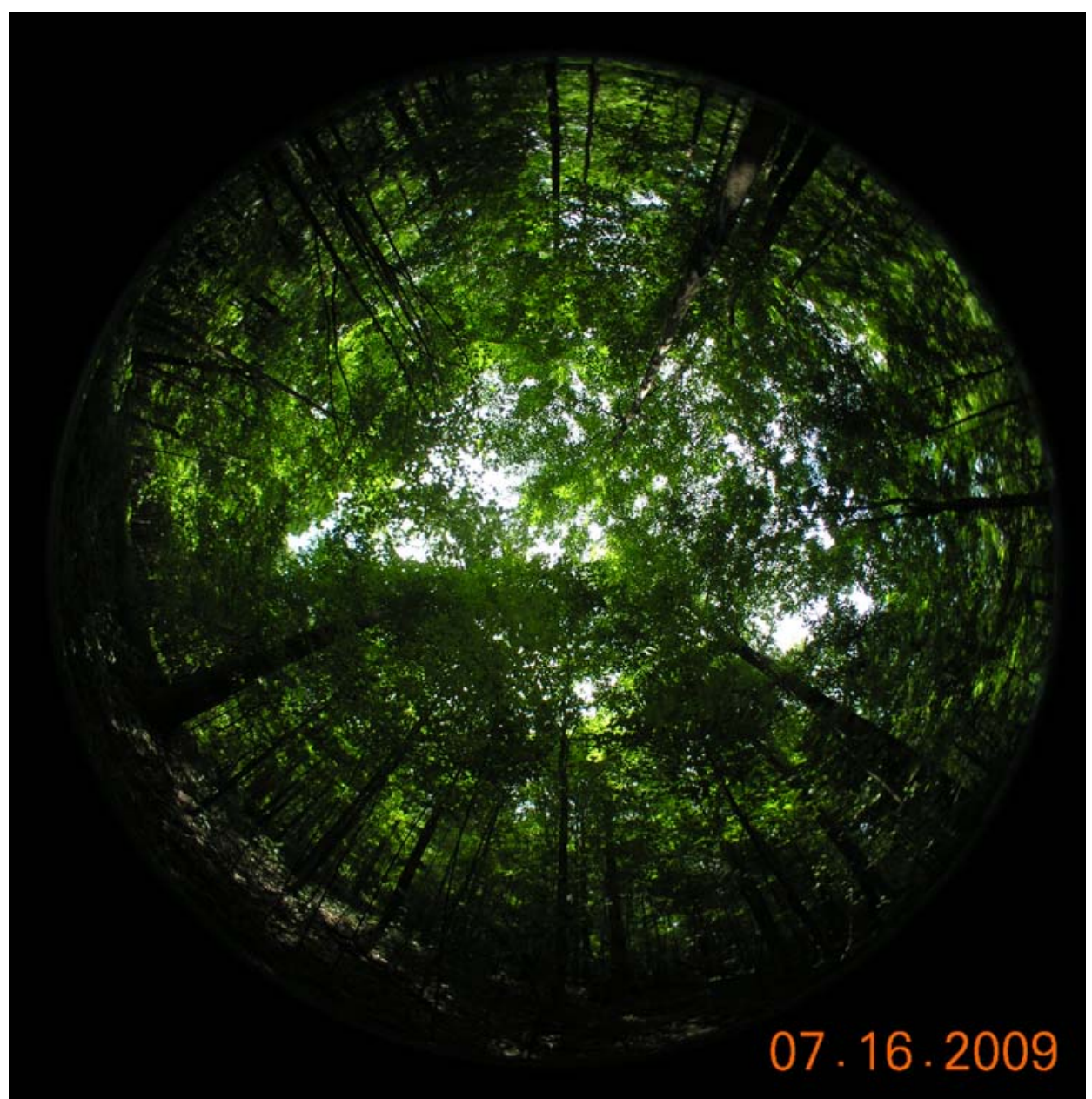

Dense canopy cover at Patch 16-1 


\section{2) Patch $17 \mathrm{~A}$}

Patch $17 \mathrm{~A}$ is a skid road that leads to a site where fluids from a gas drilling operation were dumped in 2007. Trifolium stoloniferum disappeared from this site in 2005 after peaking at 25 rooted crowns in 2003. The site is more saturated with water than other sites and muddier. Total understory vegetation cover was the lowest of any site at $18.04 \%$. The basal area of $120 \mathrm{ft}^{2} / \mathrm{ac}$ was slightly above the average of Crowngroup $0\left(112 \mathrm{ft}^{2} / \mathrm{ac}\right)$.

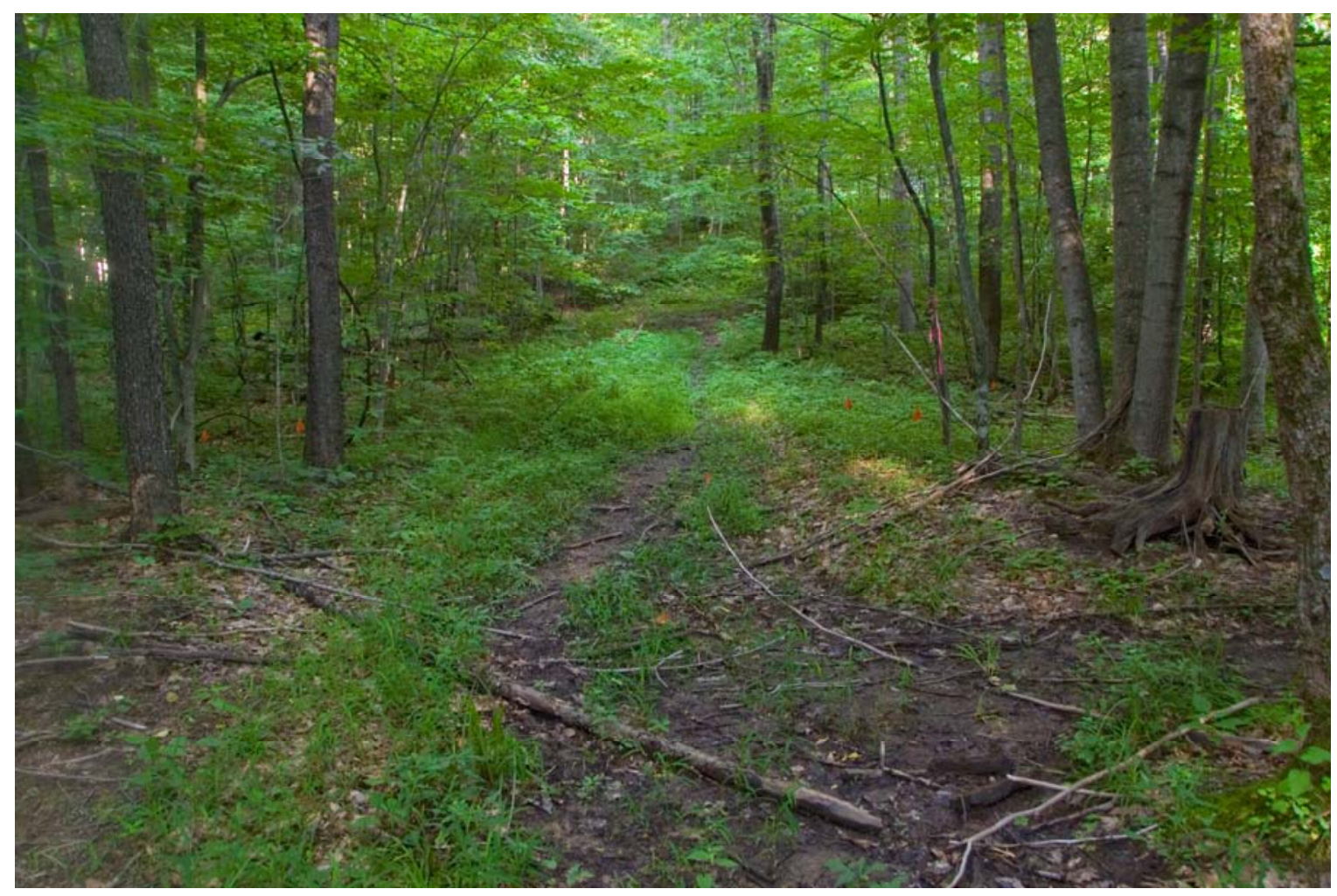

Vegetation at Patch $17 \mathrm{~A}$ demonstrating recent disturbance but with dense cover. 
Light conditions at 17A appeared to be appropriate for the growth and reproduction of $T$. stoloniferum- photosynthetically active radiation was measured at $11.37 \mu \mathrm{mol}$ photons $/ \mathrm{m}^{2} /$ second and canopy openness was at $12.37 \%$. Both of these values are similar to the means of Flowergroup 3 sites, which are those patches with a flowering index $>0.20$. So, it must be that the waterlogged nature of the site plus the heavy disturbances from the gas fluid trucks have inhibited the growth of $T$. stoloniferum at this site.

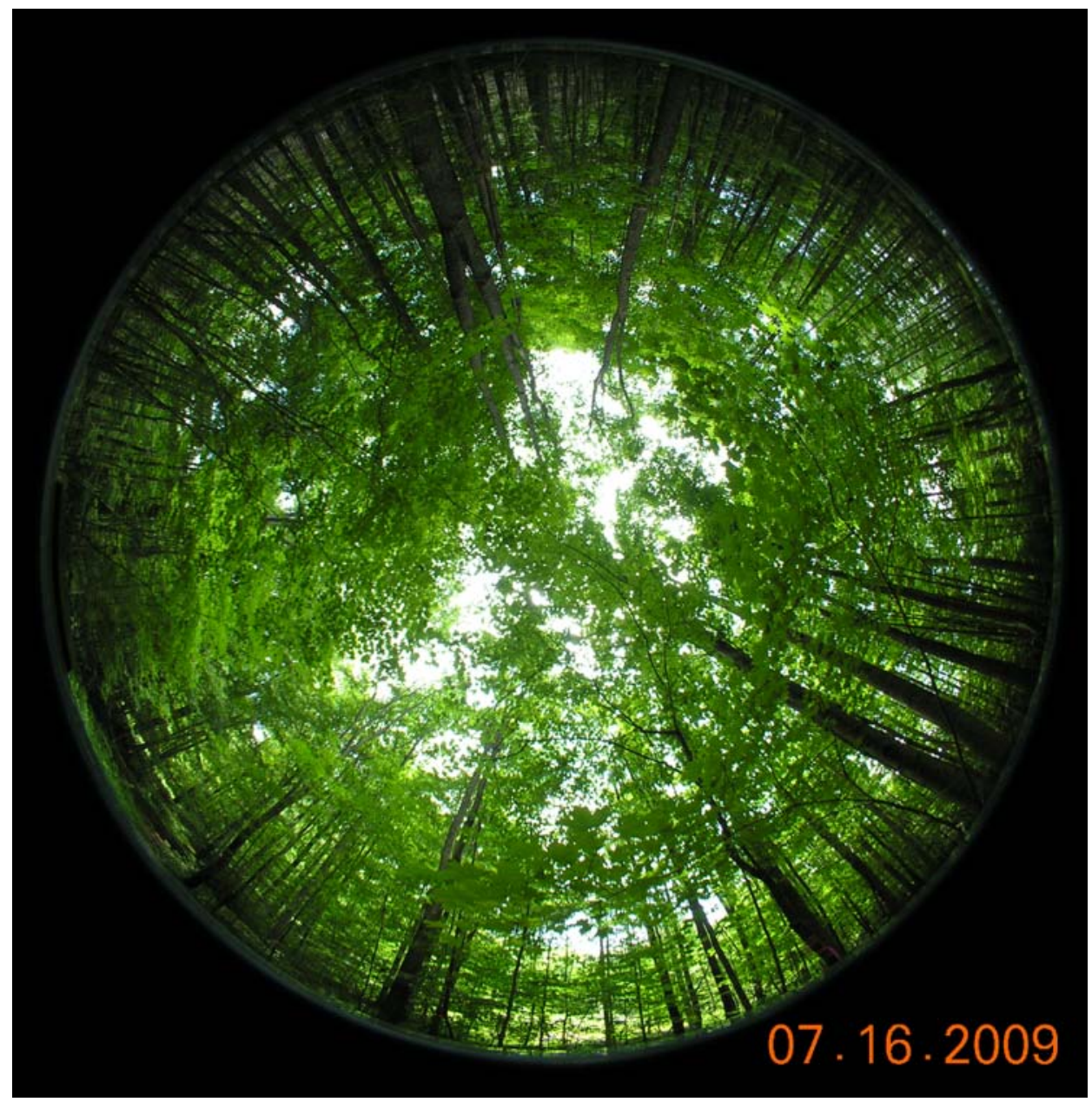

Canopy at 17-A 


\section{3) BSG5}

BSG5 might be the center of Laportea canadensis abundance in the world. Throughout this site, this species was dominating the growing space. BSG5 is located in a portion of the Fernow known as the Biological Control Area, which serves as a reference, undisturbed site. Consequently, this site has not been disturbed since management was initiated at the Fernow. Trifolium stoloniferum was recorded at this site back in 1999 with an abundance of 65 rooted crowns. There is a cave- Big Springs Cave- going downhill from BSG5, and the T. stoloniferum occurrence was located along the foot path that cavers and biologists used to access the cave. Only 2 rooted crowns of $T$. stoloniferum were recorded in 2005 and no crowns have been found since.



Vegetation at BSG5 demonstrating abundant growth of Laportea canadensis.

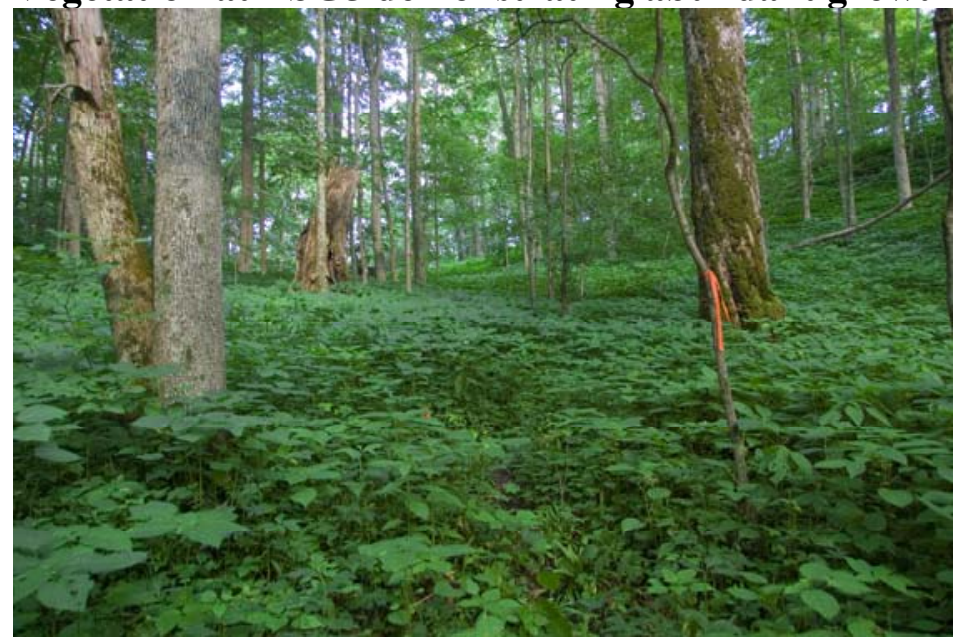

Vegetation looking uphill at BSG5 
Why has T. stoloniferum disappeared from this site? The light environment is not unfavorable to the growth of $T$. stoloniferum at $8.84 \mu \mathrm{mol}$ photons $/ \mathrm{m}^{2} /$ second and $9.4 \%$ canopy openness (these are similar to the means of Flowergroup 2 sites, which are moderately successful at flowering). The profusion of L. canadensis appears to be responsible- the instrument I used to measure light is at approximately 1-1.3 meters above ground-level. Laportea canadensis and the other tall herbs at this site, such as Impatiens spp., grow below the level of the camera, so they usurp the light resources that are conducive for $T$. stoloniferum growth and reproduction. This phenomena is similar to how the cessation of mowing in cemeteries and wildlife openings also leads to the decline of $T$. stoloniferum. In addition, the path to the cave on which T. stoloniferum was found has become overgrown due to lack of use.

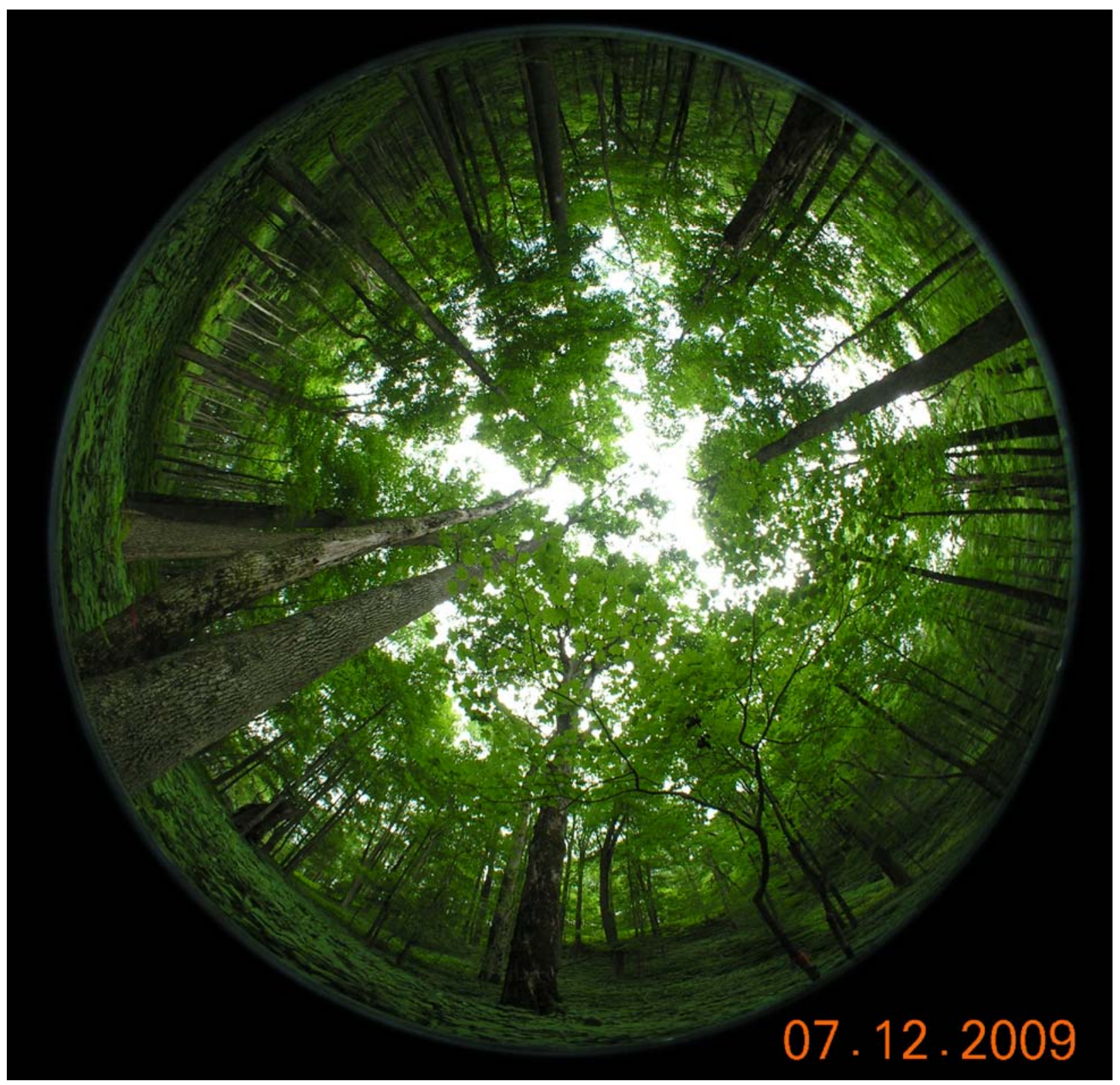

Canopy conditions at BSG5 


\section{4) Control 14-2}

Control 14-2 is a site representative of those sites that have never had $T$. stoloniferum. It occurs on a skid road (the skid road is difficult to see in the photograph because it had not been used in many years) and it is underlain by calcareous soil but there are no T. stoloniferum sites in Compartment 14. One reason that $T$. stoloniferum is absent are the limited opportunities for dispersal of $T$. stoloniferum propagules to the site, as no management activities have occurred in the compartment and thus no seeds or other propagules have been carried on logging equipment. The basal area is relatively high $\left(140 \mathrm{ft}^{2} / \mathrm{ac}\right)$ and vegetative cover is relatively low: both of these qualities are characteristic of the control sites which do not support $T$. stoloniferum at the Fernow.
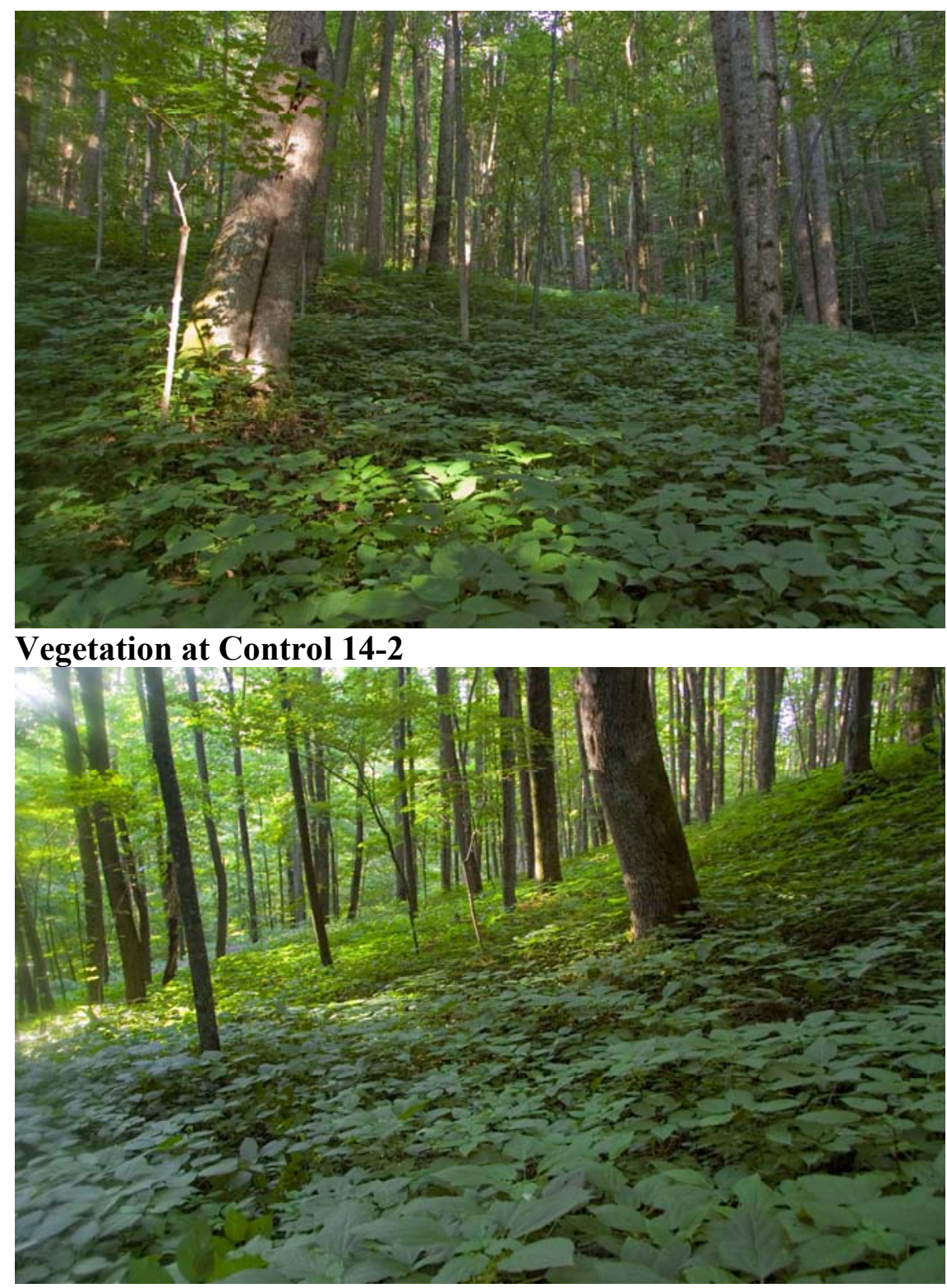

Vegetation at Control 14-2 
Control 14-2 has a light environment and canopy structure $\left(7.41 \mu \mathrm{mol}\right.$ photons $/ \mathrm{m}^{2} / \mathrm{second}$ and 9.02\% canopy openness) very much in accord with other control sites (mean $7.48 \mu \mathrm{mol}$ photons $/ \mathrm{m}^{2} /$ second and $9.13 \%$ canopy openness. In addition, it shares the species-poor understory of other control sites. As mentioned in the main body of this chapter, there were no meaningful differences between those sites that had at one time supported T. stoloniferum and those that have never supported the species. Consequently, I believe my selection of control sites was appropriate. Sites that are not suitable for T. stoloniferum despite meeting what appear to be basic environmental requirements are in general sites with dense canopies and undisturbed forest floors. These forest floors are often relatively species poor and have lower vegetative cover than sites that support T. stoloniferum.

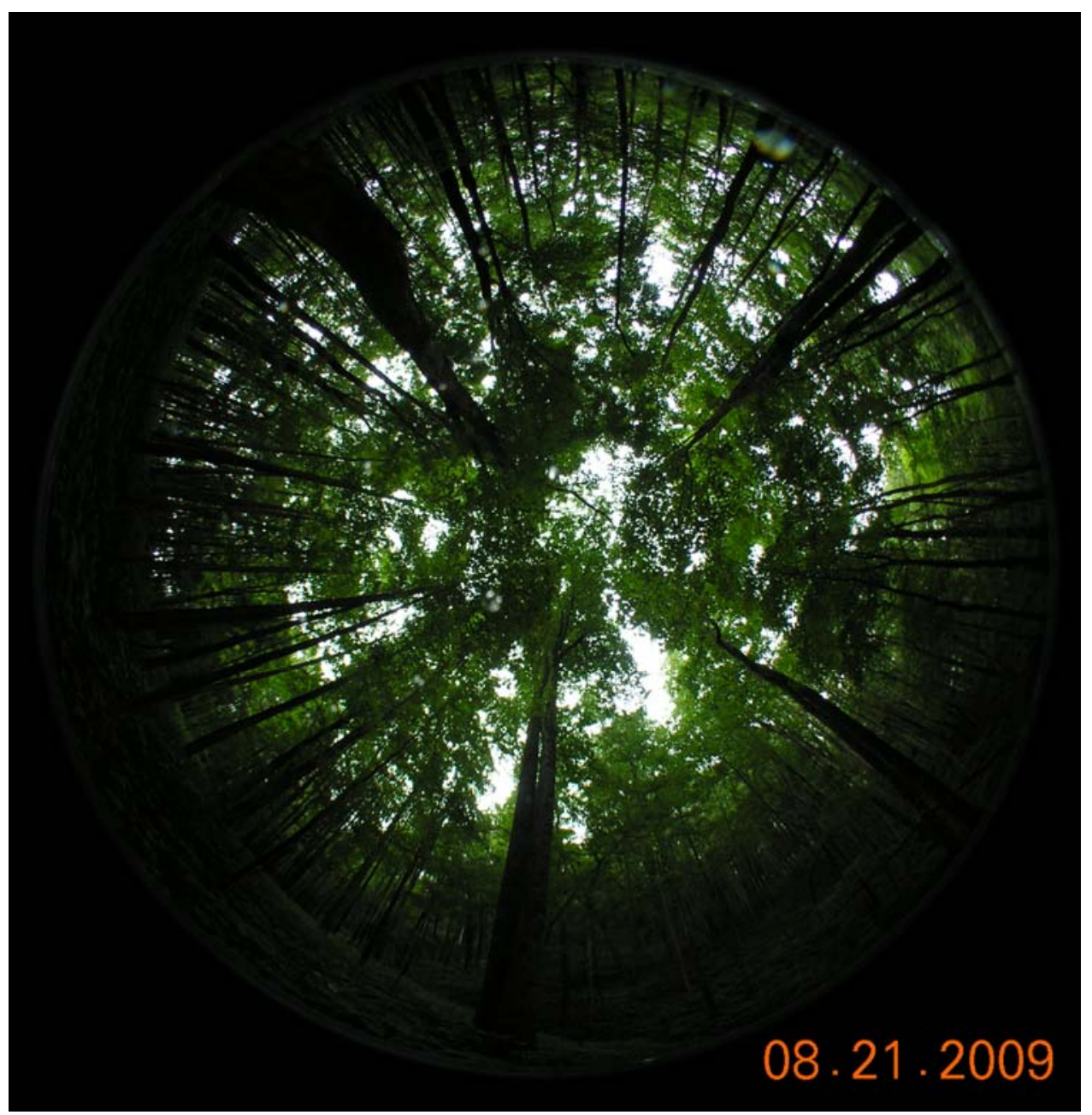

Canopy conditions at Control 14-2 


\title{
Chapter IV
}

\section{Summary of findings and implications for management of Trifolium stoloniferum}

\begin{abstract}
Logging-related disturbances are critical in maintaining Trifolium stoloniferum in its forest habitat at the Fernow Experimental Forest. The results of statistical analyses presented in Chapters II and III confirm that disturbance and associated changes to vegetation composition and structure promote $T$. stoloniferum by providing resources necessary for reproduction and population increase. Additionally, disturbance, which was primarily in the form of tree harvesting and associated skidding of logs from the forest, allows for the development of a vigorous and diverse shrub and herb layer, of which $T$. stoloniferum is a part. Disturbance that occurs too infrequently will be insufficient in maintaining habitat conditions conducive to the establishment and growth of T. stoloniferum: these conditions include perforated canopies that allow for high levels of light to reach the forest understory, an absence of a vigorous tree regeneration layer such as would be found in a clearcut or large forest gap, a vigorous herb and shrub layer, and admixed or otherwise disturbed soils. Trifolium stoloniferum sexual reproduction was most influenced by light levels, with the most successfully flowering plants having the highest measured light levels. Measured substrate variables had no effect upon $T$. stoloniferum reproduction or abundance. These findings corroborate the canon of scientific literature suggesting that openings and canopy disturbance promote the growth and reproduction of understory plants and stimulate increases in diversity by freeing resources, especially light, for herbaceous plants; however, openings above a size threshold often result in even-aged forest initiation. The majority of disturbances that have promoted T. stoloniferum throughout its range
\end{abstract}


have been incidental to its conservation or maintenance. I briefly explore the concept of incidental conservation, in which human activities generate positive conservation outcomes with specific conservation goals being absent from or incidental to the original intention of the activity. Lessons learned from the incidental conservation of this endangered species at the Fernow Experimental Forest will be applicable to the intentional and incidental conservation of T. stoloniferum throughout the Appalachian distribution of this species and perhaps its entire range.

Keywords: Incidental conservation, logging, disturbance, light, forest understory, patch dynamics.

\section{Introduction and synthesis of findings}

The questions guiding my research into the ecology of Trifolium stoloniferum Muhl. ex Eaton initially were "What effects does disturbance have upon T. stoloniferum, and how does disturbance interact with other parameters such as aspect to affect the density and presence/absence of T. stoloniferum?" These questions were addressed in Chapter II of this thesis. Classification tree analysis identified that total number of disturbances was most important in determining T. stoloniferum density within a stand (see Figures 2 and 3, Chapter II). Regression tree analysis identified aspect as most important in determining abundance within a forest compartment, and time since last disturbance was also important in distinguishing between stands that contained thriving subpopulations of T. stoloniferum and stands in which it was merely persisting (Figure 4, Chapter II). Stands with west-facing aspects that had been disturbed more recently than 14 years contained the highest densities of T. stoloniferum. Aspect was probably important because it interacted with forest canopy condition to influence light environment. Discriminant function analysis identified increases in time since last disturbance 
and proportion of basal area removed in last disturbance as highly associated with $T$. stoloniferum absence from a compartment; conversely, increases in total disturbances in a compartment were strongly associated with $T$. stoloniferum presence within a compartment. The results of this initial investigation emphasized the role of disturbance in maintaining and promoting T. stoloniferum within a compartment.

The second set of questions I asked were related to understanding T. stoloniferum success at the patch level. I asked "What environmental conditions promote patch abundance and flowering success?" and "What are the relationships between community structure and composition and T. stoloniferum success?" The results of my study found that disturbance to the canopy creates environmental conditions favorable to understory herbs and shrubs, including $T$. stoloniferum, by means of creating canopy gaps and promoting light penetration to the shrub and herbaceous layer. Trifolium stoloniferum was limited to disturbed paths at the Fernow Experimental Forest, with the majority of occurrences along skid roads. However, substrate conditions were less useful in distinguishing among patch abundance or reproductive success: litter and substrate conditions do not appear to affect $T$. stoloniferum as long as some disturbance to the soil or substrate has occurred. In other words, the range of substrate conditions that I measured at the Fernow was not systematically associated with differences in $T$. stoloniferum success.

Trifolium stoloniferum demonstrated greatest flowering success in the highest light conditions that I measured. High light environments within a forested setting are often associated with tree regeneration, but tree regeneration appeared to be suppressed by the vigorous herb layers growing in the moderately-sized gaps created by the uneven-aged silvicultural practices that promoted $T$. stoloniferum success at the stand level. In addition, it 
appeared that tree regeneration was suppressed along roads and paths by the moderate levels of disturbance to the soil, and that many gap-favoring herbs were more tolerant of these disturbances (for a striking example of this phenomenon see Appendix 2 Patch 9-46). The combination of high light and tree sapling inhibition were particularly favorable for the formation of diverse herb communities that achieved high levels of cover. Royo et al. (2010), who performed part of their study at the Fernow Experimental Forest, also suggested that preferential browsing of tree seedlings and saplings by deer might also play a part in maintaining herb cover in small disturbed gaps.

\section{Management recommendations}

The management recommendations I offer are not complex, but they could be complicated by the pervasive influence of invasive species within the deciduous forests of the eastern United States. Trifolium stoloniferum has these habitat preferences: 1) periodic disturbance-preferably more often than every 14 years (see Figure 4 of Chapter II or Figure 10 of Chapter III); 2) soil disturbance that may or may not be associated with canopy disturbance; 3) high light environments that are created by either topographic position or canopy gap formation or a combination thereof; 4) control of woody competition and periodic control of tall competitive herbs by mowing, grazing, skidding, or potentially fire; and 5) mesic, generally forested environments with calcareous soils or other soils that are not strongly acidic.

To create conditions favorable to this species, I suggest a program of limited access to $T$. stoloniferum-containing sites by horses or other large, grazing domestic animals, ATV's, foot traffic, and vehicular traffic including logging equipment. In addition, if light levels appear to be dropping to levels too low to support the establishment and growth of a vigorous herb layer at a known $T$. stoloniferum site, they could be increased by girdling or removing several trees that are 
controlling light resources at the canopy level. Aspect is an important consideration, as less tree removal may be necessary on western aspects that receive high quantities of light as indicated by my findings. All of the activities I suggested that may provide the necessary soil disturbances for T. stoloniferum pose the risk of damaging soils by means of erosion or compaction and are vectors for the spread of invasive species. These risks must be managed irrespective of the presence of T. stoloniferum.

Active management is often an effective conservation strategy, and has been proven many times in real-world conservation situations (Groom et al. 2005, McCarthy \& Possingham 2007). Active management strategies may include reintroduction, habitat restoration or manipulation, or a variety of other mechanisms used to directly alter the structure and composition of an ecological community or landscape. In many ways, habitat protection is also an active discipline, since the protection of ecosystems and communities requires the identification and prioritization of conservation objectives (Noss 1987) and the subsequent implementation of a conservation strategy.

Active management for $T$. stoloniferum entails locating occurrences, monitoring populations, and manipulating the habitat in a way that would benefit or ensure the success of this species. However, in many situations, the habitat manipulations that favored this species, which may have been critical to its persistence when it was presumed extinct, were incidental to its conservation or were conducted without full knowledge of the consequences for this diminutive species. These manipulations include logging at the Fernow Experimental Forest and Crouch Knob (which is the largest occurrence of T. stoloniferum in WV), artillery activities and cattle grazing at the Bluegrass Army Depot in Kentucky, and many other small T. stoloniferum occurrences which have been maintained by mowing, human or animal traffic, and vehicular 
traffic (USFWS 2007). What will be most essential for the effective use of incidental management is applying localized knowledge of where appropriate disturbances interact with appropriate habitat to create favorable conditions for T. stoloniferum.

The role of science in the conservation of this species appears to be to identify potential and actual habitats for T. stoloniferum and to encourage practices that are already maintaining healthy populations, intervene in the management of declining populations, and potentially to reintroduce $T$. stoloniferum to habitats that meet requirements of disturbance regime, vegetation structure, and disturbed, limestone derived soils (or soils derived from other types of calcareous bedrock).

In general, the disturbances that have created and maintained appropriate conditions for T. stoloniferum should not be stopped or diverted, as these will ultimately lead to the decline of that occurrence. In addition, active management in the form of selective reintroduction to suitable yet uncolonized sites should be considered. Seeds or other propagules could be taken from geographically or ecologically proximate sites and introduced to sites that have the appropriate combination of periodic disturbance to the soil, high light environments, low possibility of rapid tree regeneration such as small gaps and trails, and calcareous soil. I am recommending the judicious use of this practice; perhaps, reintroduction could be initiated in the setting of a designed experiment. It is possible that the liberal dispersion of seeds throughout the landscape could weaken local populations by means of disrupting local adaptations, but with the thoughtful and careful use of planned reintroduction this risk would be minimized (Moritz 1999).

As Tear et al. (2005) have suggested, conservation objectives must be clear, measurable, scientifically sound and separate from political feasibility, follow the scientific method, and anticipate change. With reference to T. stoloniferum, it is clear that the conservation goals of this 
species, as they are outlined in the USFWS Recovery Plan (2007), are to identify what ecological conditions constitute "good" T. stoloniferum habitat, preserve processes and landscapes that are maintaining and capable of maintaining good habitats, and expand these same processes so that T. stoloniferum can become an important member of the ecological communities in which it is found. The current ecological role of this species appears to be relatively limited, but based upon ecological and historical evidence there is reason to believe that its importance was once much greater. The conservation of $T$. stoloniferum will most likely be more successful if it is integrated into a process of restoring various forms of disturbance as ecological processes to the landscape.

Disturbance is an essential ecological process (Pickett \& White 1986); however, disturbance is most definitely not a conservation panacea. As I have mentioned, there are problems associated with human-mediated intervention in the environment. Invasive species appear to be a particularly troublesome and conflicting consequence of disturbance with respect to T. stoloniferum conservation and management. Microstegium vimineum (Trin.) A. Camus was among the more abundant herbaceous species associated with T. stoloniferum (see Table 12 Chapter III) But human involvement is inevitable in ecological processes during the Anthropocene (Crutzen 2006). Not only must our knowledge be as insightful and timely as possible, but our management decisions must be cognizant of human impacts, both direct and indirect. Perhaps more importantly, management at the policy and application level must synthesize cultural desire, technical knowledge, and conservation ideals in order to achieve durable and laudable results. In this situation, the desired result is the long-term persistence of $T$. stoloniferum within the context of the contemporary and future environment. 


\section{Conclusions}

The conservation of Trifolium stoloniferum should be coupled to as many other land-use and conservation goals as possible, and the role of incidental management of this species should be recognized and fostered. Whenever possible, the ecological context of Trifolium stoloniferum within a landscape should be considered and management decisions adjusted accordingly to promote the species. For T. stoloniferum, this means the creation or maintenance of high-light, disturbed-soil environments within the context of intact forest. In the Fernow Experimental Forest, these conditions were largely created and maintained by uneven-aged silviculture and associated logging and skidding.

\section{Literature cited}

Angermeier PL (2000) The natural imperative for biological conservation. Conservation Biology 14(2):373-381.

Crutzen P (2006) Part I: The Anthropocene. pgs. 13-18 in Earth System Science in the Anthropocene. Springer.

Groom MJ, Meffe GK \& CR Carroll (2005) Principles of Conservation Biology, Third Edition. Sinauer Associates.

McCarthy MA \& HP Possingham (2007) Active adaptive management for Conservation. Conservation Biology 21(4):956-963.

Moritz C (1999) Conservation units and translocations: Strategies for conserving evolutionary processes. Hereditas 130(3):217-228.

Noss RF (1987) From plant communities to landscapes in conservation inventories: A look at The Nature Conservancy (USA). Biological Conservation 41(1):11-37.

Pickett STA \& PS White (1986) The ecology of natural disturbance and patch dynamics. Academic Press, San Diego, CA.

Royo AJ, Collins R, Adams MB, Kirschbaum \& WP Carson (2010) Pervasive interactions between ungulate browsers and disturbance regimes promote temperate forest herbaceous diversity. Ecology 91(1):93-105. 
Tear TH, Kareiva P, Angermeier PL, Comer P, Czech B, Kautz R, Landon L, Mehlman D, Murphy K, Ruckelshaus M, Scott JM \& Wilhere G (2005) How much is enough? The recurrent problem of setting measurable objectives in conservation. BioScience 55(10):835-849.

United States Fish and Wildlife Service (2007) Running buffalo clover (Trifolium stoloniferum) Recovery Plan: First revision. U. S. Fish \& Wildlife Service, Fort Snelling, MN. 